\title{
Stabilization of Flows Through Porous Media
}

\author{
Von der Fakultät Mathematik und Physik \\ der Universität Hannover \\ zur Erlangung des Grades eines \\ DOKTORS DER NATURWISSENSCHAFTEN \\ Dr. rer. nat. \\ genehmigte Dissertation \\ von
}

M. Sc. Zhaoyong Feng

geboren am 28 August 1977 in Guangdong, China

2006 
Referent: Prof. Dr. J. Escher

Korreferent: Prof. Dr. G. Starke

Tag der Promotion: 26. Juni, 2006 


\section{Zusammenfassung}

Transportphänomene in porösen Medien treten in vielen ingenieurwissenschaftlichen Disziplinen auf. Im Bauingenieurwesen studiert man z.B. Stömungen in wasserführenden Schichten, die Befeuchtung technischer Strukturen oder den Transport von Schadstoffen im Boden. In der Agrartechnik wird der Transport von Wasser und Nährstoffkonzentrationen im Erdreich untersucht. Wärme- und Massenflüsse, sowie Trocknungsprozesse führen in der chemischen Verfahrenstechnik zu Transportphänomenen durch poröse Medien. Schließlich untersucht man in der Mineralölfördertechnik den Transport von Erdöl, Wasser und Gas in porösen Strukturen. Allgemein ist man an Modellen interessiert, in welchen verschiedene Größen wie Masse, chemische Konzentrationen oder Impuls durch ein poröses Medium transportiert werden. Oft enthält das poröse Medium mehrere Flüssigkeitsphasen, die gleichzeitig transportiert werden. Die Trennschichten zwischen den verschiedenen Phasen können sich frei bewegen, und sind deshalb als unbekannte Größe zu betrachten. Dies bedeutet, dass Transportphänomene in porösen Medien mathematisch durch freie Randwertaufgaben beschrieben werden, und die entsprechenden partiellen Differentialgleichungen in zeitlich sich verändernden Gebieten zu lösen sind. Außerdem erzeugen freie Ränder stets eine inhärente nichtlineare Struktur, da für diese Systeme offensichtlich kein Superpositionsprinzip für Lösungen gelten kann.

In dieser Arbeit wird der Fluss einer inkompressiblen homogenen Newtonschen Flüssigkeit in einem starren porösen Medium betrachtet. In dem unbeschränkten Medium befindet sich eine fixierte Schicht, die für die Flüssigkeit undurchlässig ist und eine externen Quelle trägt, über welche das System gesteuert werden kann. Der obere Rand ist frei und bewegt sich aufgrund der Gravitationskraft. Das Hauptanliegen dieser Arbeit besteht in einer Stabilitätsanalyse der Gleichgewichtslage des oben beschriebenen freien Randwertproblems.

Im ersten Teil der Arbeit werden die mathematischen Grundlagen zusammengestellt, die zur Untersuchung des betrachteten Problems benötigt werden. Es werden geeignete Funktionenräume eingeführt und die Theorie der maximalen Hölderregularität erklärt. Danach werden die physikalischen Grundlagen der Modellierung von Flüssen durch poröse Medien erläutert und die daraus hervorgehenden Modelle vorgestellt. Die mathematische Untersuchung der entsprechenden freien Randwertaufgabe beginnt mit der Transformation auf ein festes Referenzgebiet. Eine Reduktion des vollen Systems führt dann auf eine nichtlineare Evolutionsgleichung, die nur noch den freien Rand beinhaltet. Schließlich werden die Theorien der Fouriermultiplikatoren, der analytischen Halbgruppen, der maximalen Regularität und das Prinzip der linearisierten Stabilität angewendet, um diese Evolutionsgleichung zu untersuchen. Im Hauptresultat dieser Arbeit wird gezeigt, dass die eindeutig bestimmte Gleichgewichtslage des Systems asymptotisch stabil ist, falls die Injektionsrate in der Nähe der Gleichgewichtslage monoton wächst.

Stichwörter: Freie Ränder, Fluss durch poröse Medien, Gesetz von Darcy, voll 
nichtlineare Evolutionsgleichungen, analytische Halbgruppen, maximale Regularität, Stabilität. 


\section{Abstract}

Phenomena of transport in porous media are encountered in many engineering disciplines. Civil engineering deals, for example, with the flow of water in aquifers, the movement of moisture through engineering structures, transport of pollutants in aquifers and the propagation of stresses under foundations of structures. Agricultural engineering deals with the movement of water and solutes in the root zone in the soil. Heat and mass transport in packed-bed reactor columns and drying processes are encountered in chemical engineering. Reservoir engineers deal with the flow of oil, water and gas in petroleum reservoirs. One is interested in models in which various quantities, such as mass or momentum, through a porous medium. Often the porous material contains several fluid phases and the various quantities are transported simultaneously throughout the multiphase system. In general, the interface between different phases can move freely and are thus unknown. This means that these transport problems in porous media are mathematically described by free boundary problems, and the corresponding partial differential equations have to be solved in non-constant time depending domains. Moreover, the free interface causes an inherent nonlinear structure of these systems, since it prevents the existence of a superposition principle of solution.

This thesis considers the motion of an incompressible homogeneous Newtonian fluid in a rigid porous medium of infinite extent. This fluid is bounded below by a fixed layer having an external source, and above by a free surface moving under the influence of gravity. The main focus of this thesis is to analyze the stability of the equilibria of the moving boundary problem.

In this thesis, we first introduce some preliminary material. We give a precise definition of all function spaces needed for our analysis, as well as an introduction in the theory of maximal Hölder regularity. Then we give a short introduction on modelling of flows through porous media and describe the problem which we consider. After this, we transform the considered problem into a nonlinear evolution equation for the free interface only. Finally, we mainly use the theory of Fourier multipliers, analytic semigroup, maximal regularity and principle of linearized stability to investigate the nonlinear evolution equation. Our main result shows that if the injection rate is strictly increasing in a neighborhood of the unique equilibrium, then this equilibrium is asymptotically stable.

Keywords: Moving Boundaries, Flow through Porous Media, Darcy's Law, Fully Nonlinear Evolution Equations, Analytic Semigroups, Maximal Regularity, Stability of equilibria, Stabilization. 



\section{Contents}

1 Introduction $\quad 1$

2 Preliminary I: Function Spaces $\quad 4$

2.1 Some Function Spaces . . . . . . . . . . . . . . . . . 4

2.1.1 Bounded Functions . . . . . . . . . . . . . . . . . 4

2.1.2 Continuous Functions . . . . . . . . . . . . . . . . 4

2.1.3 Hölder Continuous Functions . . . . . . . . . . . . . 5

2.1.4 Smooth Functions . . . . . . . . . . . . . . . 6

2.1.5 Little Hölder Spaces . . . . . . . . . . . . . . . . . . . 7

2.1.6 Functions With Compact Supports . . . . . . . . . . . 7

2.1.7 Integrable Functions . . . . . . . . . . . . . . . . . . . 8

2.1.8 Distributions and Sobolev Spaces . . . . . . . . . . . . . . 9

2.2 Fourier Multipliers . . . . . . . . . . . . . . . . . . 10

2.2.1 Generalities . . . . . . . . . . . . . . . 10

$2.2 .2 \quad L_{p}$-Multipliers . . . . . . . . . . . . . . . . . . 11

$2.2 .3 \quad L_{1}$-Multipliers . . . . . . . . . . . . . . . . . . . . 11

2.3 Besov Spaces . . . . . . . . . . . . . . . . . . . . . 13

2.3.1 A Partition of Unity . . . . . . . . . . . . . . . . . . 13

2.3.2 Definition of Besov Spaces . . . . . . . . . . . . . . 13

2.3.3 The Mikhlin-Hörmander theorem on Besov spaces . . . . . . . . 13

2.4 Interpolation Theory . . . . . . . . . . . . . . . . . 16

2.4.1 Interpolatory Inclusion . . . . . . . . . . . . . . . . . 16

2.4 .2 The $K$-method . . . . . . . . . . . . . . . . . . 18

2.4.3 The trace method ................... 22

2.4.4 The Reiteration Theorem . . . . . . . . . . . . . . . 27

3 Preliminary II: Maximal Hölder Regularity 29

3.1 Analytic Semigroup . . . . . . . . . . . . . . . . . . . . . . . . . . . . . . . 39

3.1.1 Basic properties of $e^{t A} \ldots \ldots \ldots \ldots$. . . . . . . . . 31

3.1.2 Identification of the generator . . . . . . . . . . . . . 33

3.1.3 A sufficient condition to be a sectorial operator . . . . . . . . . 34

3.1.4 Intermediate spaces . . . . . . . . . . . . . . . . 36

3.2 Maximal Regularity . . . . . . . . . . . . . . . . . 43 
3.2.1 Solutions of linear problems ................ . . 44

3.2.2 Mild solutions . . . . . . . . . . . . . . . . . . 46

3.2.3 Time regularity . . . . . . . . . . . . . . . . . 50

3.2 .4 Space regularity . . . . . . . . . . . . . . . . 58

3.2.5 A further regularity result . . . . . . . . . . . . . . . . 65

3.3 Fully nonlinear equation . . . . . . . . . . . . . . . . . . 66

3.3.1 Local existence, uniqueness and regularity . . . . . . . . . . 67

3.3.2 The maximally defined solution . . . . . . . . . . . . 72

4 Stabilization of flows through porous media $\quad \mathbf{7 4}$

4.1 Modelling of flows through porous media . . . . . . . . . . . . . . . 74

4.1.1 The mass balance equations . . . . . . . . . . . . . . . . 74

4.1.2 The momentum balance equation . . . . . . . . . . . . 77

4.1.3 Darcy's law . . . . . . . . . . . . . . . . . . . . . . . . . 78

4.1.4 The boundary conditions . . . . . . . . . . . . . . . 79

4.2 The full system . . . . . . . . . . . . . . . . . . . . . . . . . . . . . . . . . . . . . . . . . . 80

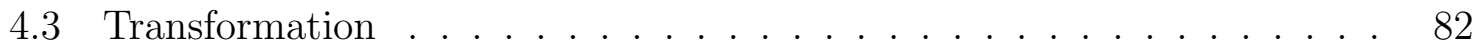

4.3.1 The diffeomorphism . . . . . . . . . . . . . . . . 82

4.3.2 The transformed operators . . . . . . . . . . . . . . 83

4.3.3 Isomorphisms induced by elliptic boundary value problems . . . 86

4.4 Reduced equation . . . . . . . . . . . . . . . . . . . . . . . . . . . . . . . . 88

4.4.1 Equivalent formulation . . . . . . . . . . . . . 88

4.4 .2 The linearization . . . . . . . . . . . . . . . . . . . . . 90

4.4.3 Fourier multipliers . . . . . . . . . . . . . . . 90

4.5 Sectorial operator . . . . . . . . . . . . . . . . . . . . . . . . . . . . . . . . . . . .

4.5.1 Spectral properties . . . . . . . . . . . . . . . . . . . . . 92

4.5.2 Estimates of the symbol . . . . . . . . . . . . . . 97

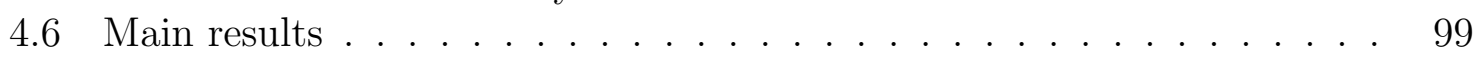

4.6.1 Stability of the transformed problem . . . . . . . . . . . . 99

4.6.2 Stability estimates for the interface and pressure . . . . . . . . . 101

$\begin{array}{ll}\text { Bibliography } & 106\end{array}$ 


\section{Chapter 1}

\section{Introduction}

In the thesis, we shall investigate the motion of an incompressible homogeneous Newtonian fluid in a rigid porous medium of infinite extent. That fluid is bounded below by a fixed layer $\Gamma_{0}$ having an external source (with an injection rate $b$ ), and above by a free surface $\Gamma_{f}$ moving under the influence of gravity, see Figure 0. Moreover, the fluid flow is governed by Darcy's law. More precisely, writing $u(t, x, y)$ for the pressure at the moment $t$ and at the position $(x, y)$ and $f(t, x)$ for the moving interface, we can describe it as the following problem:

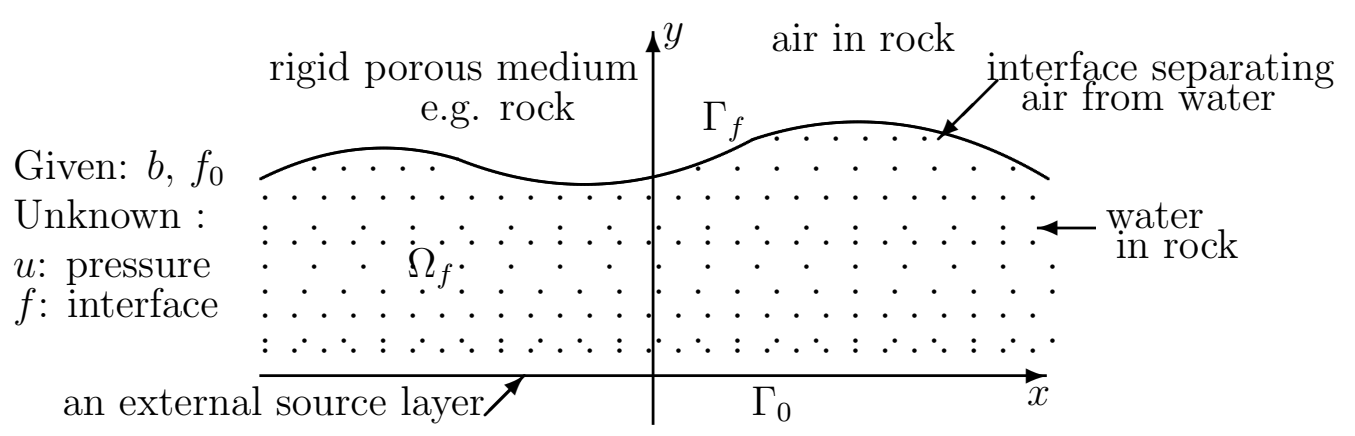

Figure 0

$(P) \quad\left\{\begin{array}{rlrl}\Delta u(t, x, y) & =0, & & t \in J,(x, y) \in \Omega_{f(t)} \\ \left.\frac{\partial u}{\partial y}(t, x, y)\right|_{y=0} & =b(f(t, x)), & & t \in J, x \in \mathbb{R} \\ \left.u(t, x, y)\right|_{y=f(t, x)} & =f(t, x), & & t \in J, x \in \mathbb{R} \\ \lim _{\mid x(t, x, y)} & =c, & & t \in J,(x, y) \in \Omega_{f(t)} \\ |x|+|y| \rightarrow \infty & & t \in J,(x, y) \in \Gamma_{f(t)} \\ \partial_{t} f(t, x)+\left(-f_{x}, 1\right) \cdot \nabla u(t, x, y) & =0, & & x \in \mathbb{R},\end{array}\right.$

where $T>0$ is fixed, and $J=[0, T), \dot{J}=J \backslash\{0\}$. Moreover,

$$
\Omega_{f(t)}:=\{(x, y) \in \mathbb{R} \times(0, \infty) ; 0<y<f(t, x)\}
$$


and

$$
\Gamma_{f(t)}:=\operatorname{graph}(f):=\{(x, y) \in \mathbb{R} \times(0, \infty) ; y=f(t, x)\} .
$$

For simplicity, we will use $\Omega_{f}$ and $\Gamma_{f}$ to replace $\Omega_{f(t)}$ and $\Gamma_{f(t)}$, respectively. The injection rate $b$ is a sufficiently smooth function from $\mathbb{R}$ to $\mathbb{R}$. Since the injection rate can be controlled in advance, we can think it is a given smooth function. Finally, we are interested in classical solutions of the problem $(P)$. Therefore $f$ has to satisfy at least the following conditions:

$$
f \in \widetilde{\mathfrak{A}}:=\left\{f \in B C^{2}(\mathbb{R}) ; \inf _{x \in \mathbb{R}} f(x)>0\right\} .
$$

Otherwise, if $f(x)=0$ for some $x$ then the interface and the fluid domain have a singularity at this point.

In [12], J. Escher and G. Simonett studied the case of the fluid bounded below by a fixed impermeable layer, i.e. $b \equiv 0$. They proved that the problem $(P)$ with $b \equiv 0$ is locally in time well-posed (cf. [1]). However, the question of the stability of the equilibrium solution $(u, f) \equiv(c, c)$ was not addressed in [12]. The main goal of this research is to specify classes of injection rate $b$ such that the equilibrium $(u, f) \equiv(c, c)$ is stable. Clearly, if $(u, f)=(c, c)$ is an equilibrium of the problem $(P)$, it is necessary to assume that

$$
b(c)=0 .
$$

In order to state our results for problem $(P)$, we need some notations. Given $m \in \mathbb{N}$, we write $\mathcal{S}\left(\mathbb{R}^{m}\right)$ for the Schwartz space, that is, the Fréchet space of all rapidly decreasing smooth functions on $\mathbb{R}^{m}$. Moreover, assume that $k \in \mathbb{N}, \alpha \in(0,1)$ and that $U$ is an open subset of $\mathbb{R}^{m}$. Then $B U C^{k+\alpha}(U)$ denote the classical Hölder spaces of functions having bounded derivatives up to order $k$, and such that the $k$-th derivatives satisfy a uniformly $\alpha$-Hölder condition, see Section 2.1 for a precise definition.

For $s>0$, we define the closed subspaces $h^{s}\left(\mathbb{R}^{m}\right)$ of little Hölder spaces buc $\left(\mathbb{R}^{m}\right)$ by

$$
h^{s}\left(\mathbb{R}^{m}\right):=\text { closure of } \mathcal{S}\left(\mathbb{R}^{m}\right) \text { in } B U C^{s}\left(\mathbb{R}^{m}\right) .
$$

Furthermore, let $\gamma_{U}$ denote the restriction operator with respect to $U$. Then we define

$$
h^{s}(U):=\text { closure of } \gamma_{U} \mathcal{S}\left(\mathbb{R}^{m}\right) \text { in } B U C^{s}(U) .
$$

For convenience, we also call $h^{s}\left(\mathbb{R}^{m}\right)$ to be little Hölder spaces. Finally, let

$$
\mathfrak{A}:=\left\{g \in B C^{2}(\mathbb{R}) ; \inf _{x \in \mathbb{R}}\{c+g(x)\}>0\right\}
$$

and for $s>0$,

$$
h^{s}:=h^{s}(\mathbb{R}), \quad \text { and } \quad h_{\mathfrak{A}}^{s}:=h^{s} \cap \mathfrak{A} .
$$

We are now ready to state the following existence result for problem $(P)$. 
Theorem 1.0.1. Assume that $b(c)=0$ and $b^{\prime}(c)>0$. Then there exists $\omega_{0}>0$, such that for any $\omega \in\left[0, \omega_{0}\right)$, there also exist positive constants $r$ and $C=C(r)$. If $f_{0}-c \in h_{\mathfrak{A}}^{2+\alpha}$ with $\left\|f_{0}-c\right\|_{h^{2+\alpha}}<r$, the problem $(P)$ has a unique global solution $(u(t, x, y), f(t, x))$ such that

$$
u-c \in C\left([0,+\infty), h^{2+\alpha}\left(\Omega_{f}\right)\right) \quad \text { and } \quad f-c \in C\left([0,+\infty), h_{\mathfrak{A}}^{2+\alpha}\right) .
$$

Furthermore, we get the following estimate

$$
\|f(t)-c\|_{h^{2+\alpha}}+\left\|f^{\prime}(t)\right\|_{h^{1+\alpha}}+\|u(t)-c\|_{h^{2+\alpha}\left(\Omega_{f(t)}\right)} \leq C e^{-\omega t}\left\|f_{0}-c\right\|_{h^{2+\alpha}}, \quad \forall t \geq 0 .
$$

A fundamental and inherent difficulty of an analytic treatment of problem $(P)$ is manifested in the fact that one has to work with variable domains. The nonlinear structure hidden behind this circumstance is clearly disclosed by the observation that there is no superposition principle for solution of the problem $(P)$. This means problem $(P)$ is a nonlinear partial differential problem. To prove our result, we first consider a transformed version of $(P)$ which applies over a fixed domain. In fact, it turns out that the transformed problem consists of a nonlinear elliptic equation and a parabolic equation with a nonlinear nonlocal pseudo-differential operator of first order. Then we solve the nonlinear elliptic equation, and reduce the transformed problem to a nonlinear evolution equation. Finally, we mainly use the theory of Fourier multipliers, analytic semigroup and maximal regularity to investigate the nonlinear evolution equation.

We mention that the corresponding set of equations for incompressible fluids in porous media was investigated in $[11,13,18,22]$. In particular, J. Escher and G. Prokert investigated spatially periodic flows in porous media in [16]. The moving boundary problem in deformable porous media was also investigated in [14, 15]. Moreover, there is a different approach in weighted Hölder spaces to that problem outlined in $[7,8]$. In addition, a $L_{p}$-theory for that problem was proposed in $[25,26]$.

\section{Acknowledgements}

This thesis was supported by the Deutsche Forschungsgemeinschaft $(D F G)^{1}$ through a scholarship in the Graduiertenkolleg 615 "Interaction of modelling, computation methods and software concepts for scientific-technological problems" at the University of Hannover. I am very grateful for this support.

I especially thank my advisor, Prof. Dr. Joachim Escher. He supported me and my work with patience, many fruitful discussions and invaluable hints.

I also thank all members of the Graduiertenkolleg for the various support I experienced in many discussions, seminars and colloquia, especially Prof. Dr. Gerhard Starke, Prof. Dr. Ernst P. Stephan and my classmate Karlheinz Kneisel. Further, I thank Prof. Shangbin Cui and Prof. Zhaoyang Yin. They gave me a chance to visit Sun Yat-Sen University in China and also provided me with helpful advice for my thesis.

At last, I thank my parents for their support and patience.

\footnotetext{
${ }^{1}$ German Research Foundation
} 


\section{Chapter 2}

\section{Preliminary I: Function Spaces}

In the first part of this chapter we fix some notations by defining some basic function spaces. We then introduce Fourier multipliers on Lebesgue and Besov spaces. We close this chapter by discussing some results from interpolation theory which we need for our approach.

\subsection{Some Function Spaces}

\subsubsection{Bounded Functions}

Let $E$ be a Banach space and suppose that $X$ is a nonempty set. Then $B(X, E)$ is the Banach space of all bounded functions $u: X \rightarrow E$, equipped with the supremum norm

$$
\|u\|_{\infty}:=\|u\|_{\infty}^{X}:=\sup _{x \in X}\|u(x)\|_{E}
$$

\subsubsection{Continuous Functions}

Let now $X$ be a metric space. We denote by $B C(X, E)$ the closed subspace of $B(X, E)$ consisting of all bounded and continuous $E$-valued functions. Moreover, $B U C(X, E)$ is the closed subspace of $B C(X, E)$ consisting of all bounded and uniformly continuous $E$-valued functions on $X$.

Of course, $C(X, E)$ is given the topology of uniform convergence on compact subsets of $X$, the compact-open topology. If $X$ is locally compact and separable, $C(X, E)$ is a Fréchet space.

If $X$ is locally compact, $C_{0}(X, E)$ is the Banach space of all continuous functions vanishing at infinity, equipped with the supremum norm. Recall that $u: X \rightarrow E$ vanishes at infinity if, given $\varepsilon>0$, there exists $K \subset \subset X$ such that $\|u(x)\|_{E}<\varepsilon$ for $x \in K^{c}:=X \backslash K$. It is easily seen that $C_{0}(X, E)$ is a closed subspaces of $B U C(X, E)$. Hence

$$
C_{0}(X, E) \hookrightarrow B U C(X, E) \hookrightarrow B C(X, E) \hookrightarrow C(X, E)
$$


and these spaces all coincide if $X$ is compact.

\subsubsection{Hölder Continuous Functions}

Let $d$ be a metric for $X$ and let $0<\rho<1$. A function $u: X \rightarrow E$ is uniformly $\rho$-Hölder continuous if

$$
[u]_{\rho}:=[u]_{\rho}^{X}:=\sup _{\substack{x, y \in X \\ x \neq y}} \frac{\|u(x)-u(y)\|_{E}}{[d(x, y)]^{\rho}}<\infty .
$$

It is (locally) $\rho$-Hölder continuous if each point has a neighborhood $Y$ such that $u \mid Y$ is uniformly $\rho$-Hölder continuous. We write $u \in U C^{\rho}(X, E)$ if $u$ is uniformly $\rho$-Hölder continuous, and

$$
C^{\rho}(X, E):=\{u: X \rightarrow E ; u \text { is } \rho \text { - Hölder continuous }\} .
$$

If $\rho$ is replaced by 1 , a [uniformly] 1-Hölder continuous function is said to be [uniformly] Lipschitz continuous and we write $U C^{1-}(X, E)$ and $C^{1-}(X, E)$ for the sets of all uniformly Lipschitz continuous functions and of all Lipschitz continuous functions, respectively, mapping $X$ into $E$. In numerical calculations the symbol 1 - will always be identified with the number 1 .

If $u \in C^{1-}(X, E)$ and $K$ is a compact subset of $X$, there exists a neighborhood of $Y$ of $K$ in $X$ such that $u \mid Y$ is uniformly Lipschitz continuous (e.g., Proposition 6.4 in [3]). It is not difficult to see that $d^{\rho}$ is an equivalent metric for $X$. Thus $u \in C^{\rho}(X, E)$ iff $u \in C^{1-}\left(\left(X, d^{\rho}\right), E\right)$. This implies that, given $K \subset \subset X$ and $u \in C^{\rho}(X, E)$, there exists a neighborhood of $Y$ of $K$ such that $u \mid Y \in U C^{\rho}(Y, E)$.

Given $\rho \in(0,1) \cup\{1-\}$, we equip $C^{\rho}(X, E)$ with the family of seminorms

$$
\|\cdot\|_{C^{\rho}}^{K}:=\|\cdot\|_{\infty}^{K}+[\cdot]_{\rho}^{K}, \quad K \subset \subset X .
$$

Then $C^{\rho}(X, E)$ is a $L C S$ (Locally Convex Space) such that

$$
C^{\rho}(X, E) \hookrightarrow C(X, E) .
$$

If $X$ is locally compact and separable, $C^{\rho}(X, E)$ is a Fréchet space.

For each $\rho \in(0,1) \cup\{1-\}$, we denote by

$$
B U C^{\rho}(X, E):=\left(B U C^{\rho}(X, E),\|\cdot\|_{C^{\rho}}\right)
$$

the Banach space of all bounded and uniformly $\rho$-Hölder continuous $E$-valued functions on $X$, where $\|\cdot\|_{C^{\rho}}:=\|\cdot\|_{C^{\rho}}^{X}$. Observe that

$$
B U C^{\rho}(X, E) \hookrightarrow B U C^{\sigma}(X, E) \hookrightarrow B U C(X, E)
$$

provided $\rho, \sigma \in(0,1) \cup\{1-\}$ satisfy $\sigma<\rho$. In particular, we write $\operatorname{Lip}(X, E)$ instead of $B U C^{1-}(X, E)$. 


\subsubsection{Smooth Functions}

Now suppose that $X$ is a nonempty open subset of $\mathbb{R}^{n}$, where $n \in \dot{\mathbb{N}}$. In the following $D_{j}:=-i \partial_{j}$ with $\partial_{j}:=\partial / \partial x_{j}$ for $j=1, \cdots, n$, and we use standard multiindex notation.

Of course, $C^{m}(X, E)$ has the usual meaning for $m \in \dot{\mathbb{N}}$, and $C^{0}:=C$. Given a nonempty open subset $Y$ of $X$,

$$
\|u\|_{C^{m}}^{Y}:=\max _{|\alpha| \leq m}\left\|\partial^{\alpha} u\right\|_{\infty}^{Y}
$$

defines a seminorm on $C^{m}(X, E)$ and

$$
C^{m}(X, E):=\left(C^{m}(X, E),\left\{\|\cdot\|_{C^{m}}^{K} ; K=\stackrel{\circ}{K} \subset \subset X\right\}\right)
$$

is a Fréchet space. If $\rho \in(0,1) \cup\{1-\}$, we put

$$
C^{m+\rho}(X, E):=\left\{u \in C^{m}(X, E) ; \partial^{\alpha} u \in C^{\rho}(X, E),|\alpha|=m\right\},
$$

equipped with the family of seminorms

$$
u \mapsto\|u\|_{C^{m+\rho}}^{K}:=\|u\|_{C^{m}}^{K}+\max _{|\alpha|=m}\left[\partial^{\alpha} u\right]_{\rho}^{K}, \quad K=\stackrel{\circ}{K} \subset \subset X .
$$

It is not difficult to see that $C^{m+\rho}(X, E)$ is a Fréchet space and

$$
C^{m+\rho}(X, E) \hookrightarrow C^{m+\sigma}(X, E) \hookrightarrow C^{m}(X, E) \hookrightarrow C^{m-}(X, E),
$$

where $\rho, \sigma \in(0,1) \cup\{1-\}$ satisfy $\sigma<\rho$ and $m-:=(m-1)+1-$ for $m \in \dot{\mathbb{N}}$. In fact, $C^{m}(X, E)$ is a closed linear subspaces of $C^{m-}(X, E)$.

Of course,

$$
C^{\infty}(X, E):=\bigcap_{m=0}^{\infty} C^{m}(X, E)=\bigcap_{s \geq 0} C^{s}(X, E)
$$

is a Fréchet space too (with respect to the obvious projective limit topology).

We write

$$
\|\cdot\|_{C^{m+\rho}}:=\|\cdot\|_{C^{m+\rho}}^{X}, \quad \rho \in[0,1) \cup\{1-\} .
$$

Then the Banach spaces $B C^{m}(X, E)$ and $B U C^{m}(X, E)$ are defined in the obvious way and are equipped with the norm $\|\cdot\|_{C^{m}}$. Moreover, given $\rho \in(0,1) \cup\{1-\}$,

$$
B U C^{m+\rho}(X, E):=\left(\left\{u \in C^{m+\rho}(X, E) ;\|u\|_{C^{m+\rho}}<\infty\right\},\|\cdot\|_{C^{m+\rho}}\right)
$$

is a Banach space too. In addition, we introduce the Fréchet spaces

$$
B C^{\infty}(X, E):=\bigcap_{m=0}^{\infty} B C^{m}(X, E)
$$

and

$$
B U C^{\infty}(X, E):=\bigcap_{m=0}^{\infty} B U C^{m}(X, E)
$$




\subsubsection{Little Hölder Spaces}

Let $X$ be a nonempty open subset of $\mathbb{R}^{n}$. It is well known that the injection

$$
B U C^{m+\rho}(X, E) \hookrightarrow B U C^{m+\sigma}(X, E), \quad m \in \mathbb{N}, \quad 0<\sigma<\rho<1,
$$

which is an obvious consequence of (2.1.5), is not dense. For this reason we introduce the little Hölder spaces:

$$
b u c^{s}(X, E):=\text { closure of } B U C^{m+1}(X, E) \text { in } B U C^{s}(X, E)
$$

for $m \in \mathbb{N}$ and $m<s<m+1$. Then $u \in b u c^{m+\rho}\left(\mathbb{R}^{n}, E\right)$ iff $u \in B U C^{m}\left(\mathbb{R}^{n}, E\right)$ and

$$
\lim _{t \rightarrow 0} \sup _{\substack{x, y \in \mathbb{R}^{n} \\ 0<|x-y| \leq t}} \frac{\left\|\partial^{\alpha} u(x)-\partial^{\alpha} u(y)\right\|_{E}}{|x-y|^{\rho}}=0, \quad \alpha \in \mathbb{N}^{n},|\alpha|=m .
$$

Further, $B U C^{\infty}\left(\mathbb{R}^{n}, E\right)$ is dense in $b u c^{m+\rho}\left(\mathbb{R}^{n}, E\right)$ for $m \in \mathbb{N}$ and $\rho \in(0,1)$.

Example 2.1.1. Let $\alpha \in(0,1)$, then $x^{\alpha} \in B U C^{\alpha}(0,1) \backslash b u c^{\alpha}(0,1)$.

Proof Clearly, $x^{\alpha}$ is bounded and continuous in $(0,1)$. To prove $x^{\alpha} \in B U C^{\alpha}(0,1)$, it is sufficient to prove that

$$
\left|x^{\alpha}-y^{\alpha}\right| \leq|x-y|^{\alpha} \quad \text { for } x, y>0 .
$$

Without loss of generality, we suppose that $x \geq y>0$. Then we define

$$
h(x)=x^{\alpha}-y^{\alpha}-(x-y)^{\alpha} \text { for } x \geq y>0 .
$$

By directly calculating, we know that $h(y)=0$ and $h^{\prime}(x)=\alpha\left(x^{\alpha-1}-(x-y)^{\alpha-1}\right)<0$. This means $h(x) \geq h(y)=0$, i.e.

$$
x^{\alpha}-y^{\alpha} \leq(x-y)^{\alpha} \text { for } x \geq y>0 .
$$

Thus (2.1.10) holds. This implies $x^{\alpha} \in B U C^{\alpha}(0,1)$.

For any $t \in(0,1)$, we can take $x=t \in(0,1)$ and $y=t / 2 \in(0,1)$, then we know that $|x-y|=t / 2<t$, and

$$
\frac{\left|x^{\alpha}-y^{\alpha}\right|}{|x-y|^{\alpha}}=\frac{t^{\alpha}-(t / 2)^{\alpha}}{(t / 2)^{\alpha}}=2^{\alpha}-1 \neq 0 .
$$

Therefore $x^{\alpha} \notin b u c^{\alpha}(0,1)$. This completes the proof.

\subsubsection{Functions With Compact Supports}

If $m \in \mathbb{N} \cup\{\infty\}$ and $\rho \in[0,1) \cup\{1-\}$, we denote by $C_{c}^{m+\rho}(X, E)$ the subspace of $C^{m+\rho}(X, E)$ consisting of all functions $u$ whose supports, $\operatorname{supp}(u)$, are compact in $X$, endowed with the usual inductive limit topology. In particular,

$$
\mathcal{D}(X, E):=C_{c}^{\infty}(X, E)
$$


is the space of $E$-valued test functions on $X$ and $\mathcal{D}(X):=\mathcal{D}(X, \mathbb{K})$, where $\mathbb{K}$ is either $\mathbb{R}$ or $\mathbb{C}$, if no confusion seems likely. Lastly,

$$
\stackrel{\circ}{C}^{s}(X, E):=\text { closure of } \mathcal{D}(X, E) \text { in } B U C^{s}(X, E), \quad s \in \mathbb{R}^{+} .
$$

It is not difficult to see that

$$
\stackrel{\circ}{C}^{m}(X, E)=C_{0}^{m}(X, E):=\left\{u \in C^{m}(X, E) ; \partial^{\alpha} u \in C_{0}(X, E),|\alpha| \leq m\right\} .
$$

\subsubsection{Integrable Functions}

Let $X$ be a $\sigma$-compact metric space and let $\mu$ be a positive Radon measure on $X$. Given $q \in(0, \infty]$, a measurable subset $Y$ of $X$, and a (strongly) measurable function $u: X \rightarrow E$, put

$$
\|u\|_{q}^{Y}:=\left\{\begin{array}{lr}
\left(\int_{Y}\|u(y)\|_{E}^{q} d \mu\right)^{1 / q}, & 0<q<\infty \\
e s s-\sup \|u(y)\|_{E}, & q=\infty .
\end{array}\right.
$$

If $q \geq 1$ then $L_{q, l o c}(X, \mu, E)$ is the Fréchet space of all (equivalence classes of strongly) measurable functions $u: X \rightarrow E$ such that

$$
\|u\|_{q}^{K}<\infty, \quad K=\stackrel{\circ}{K} \subset \subset X
$$

topologized with the family of seminorms $(2.1 .11)$. We put $\|\cdot\|_{q}:=\|\cdot\|_{q}^{X}$ and denote by

$$
L_{q}(X, \mu, E):=\left(L_{q}(X, \mu, E),\|\cdot\|_{q}\right), \quad 1 \leq q \leq \infty,
$$

the usual Lebesgue spaces, which are Banach spaces. (The fact that we use the symbol $\|\cdot\|_{\infty}$ in two different contexts will cause no confusion.) In particular,

$$
l_{p}(E):=L_{p}(\mathbb{N}, \kappa, E), \quad 1 \leq p \leq \infty,
$$

where $\kappa$ is the counting measure, are Lebesgue spaces of $E$-valued sequences.

If $E=\mathbb{K}$ and no confusion seems possible, we put $L_{p}(X, \mu):=L_{p}(X, \mu, \mathbb{K})$.

If $X$ is an open subset of $\mathbb{R}^{n}$ (more generally, a Lebesgue measurable subset of $\mathbb{R}^{n}$ ) and $\mu$ is the $n$-dimensional Lebesgue measure on $X$, we simply put

$$
L_{p}(X, E):=L_{p}(X, \mu, E), \quad 1 \leq p \leq \infty,
$$

and $d x:=d \mu$.

Let now $X$ again be an open subset of $\mathbb{R}^{n}$. Recall that

$$
\mathcal{D}(X, E) \hookrightarrow L_{q}(X, E) \hookrightarrow L_{q, l o c}(X, E) \stackrel{d}{\hookrightarrow} L_{1, l o c}(X, E), \quad 1 \leq q \leq \infty,
$$

and that the first two injections are dense too if $q<\infty$. 


\subsubsection{Distributions and Sobolev Spaces}

We write $\mathcal{D}^{\prime}(X, E)$ for the spaces of $E$-valued distributions on $X$, that is,

$$
\mathcal{D}^{\prime}(X, E):=\mathcal{L}(\mathcal{D}(X), E)
$$

equipped with the topology of uniform convergence on bounded sets, and

$$
\mathcal{D}^{\prime}(X):=\mathcal{D}^{\prime}(X, \mathbb{K})
$$

We also use $\partial_{j}\left(\right.$ and $D_{j}$ ) to denote distributional partial derivatives. Recall that

$$
L_{1, l o c}(X, E) \hookrightarrow \mathcal{D}^{\prime}(X, E)
$$

by means of the identification

$$
u(\phi):=\int_{X} \phi(x) u(x) d x, \quad \phi \in \mathcal{D}(X), \quad u \in L_{1, l o c}(X, E) .
$$

Given $m \in \mathbb{N}$ and $q \in[1, \infty]$, the Sobolev space, $W_{q}^{m}(X, E)$, of order $m$ over $L_{q}$ consisting of $E$-valued distributions on $X$ is defined by

$$
W_{q}^{m}(X, E):=\left(\left\{u \in L_{q}(X, E) ; \partial^{\alpha} u \in L_{q}(X, E),|\alpha| \leq m\right\},\|\cdot\|_{m, q}\right),
$$

where

$$
\|u\|_{m, q}:=\|u\|_{W_{q}^{m}(X, E)}:=\left\{\begin{array}{lr}
\left(\sum_{|\alpha| \leq m}\left\|\partial^{\alpha} u\right\|_{q}^{q}\right)^{1 / q}, & 1 \leq q<\infty \\
\max _{|\alpha| \leq m}\left\|\partial^{\alpha} u\right\|_{\infty}, & q=\infty .
\end{array}\right.
$$

For $q \in[1, \infty)$ and $s \in(0,1)$ we put

$$
[u]_{s, q}:=[u]_{W_{q}^{s}(X, E)}:=\left(\int_{X \times X}\left(\frac{\|u(x)-u(y)\|}{|x-y|^{s}}\right)^{q} \frac{d(x, y)}{|x-y|^{n}}\right)^{1 / q} .
$$

Moreover, we denote by $[t]$ the largest integer less or equal to $t \in \mathbb{R}$. Then, given $s \in \mathbb{R}^{+} \backslash \mathbb{N}$ and $q \in[1, \infty)$, we define the Slobodeckii space, $W_{q}^{s}(X, E)$, of order $s$ consisting of $E$-valued distributions on $X$ by

$$
W_{q}^{s}(X, E):=\left(\left\{u \in W_{q}^{[s]}(X, E) ;\left[\partial^{\alpha} u\right]_{s-[s], q}<\infty,|\alpha|=[s]\right\},\|\cdot\|_{s, q}\right),
$$

where

$$
\|u\|_{s, q}:=\|u\|_{W_{q}^{s}(X, E)}:=\left(\|u\|_{[s], q}^{q}+\sum_{|\alpha|=[s]}\left[\partial^{\alpha} u\right]_{s-[s], q}^{q}\right)^{1 / q} .
$$


Further, $W_{q}^{0}(X, E):=L_{q}(X, E)$ so that $\|\cdot\|_{0, q}=\|\cdot\|_{q}$. It is well known that the Sobolev and the Slobodeckii spaces are Banach spaces and that

$$
W_{q}^{s}(X, E) \hookrightarrow W_{q}^{t}(X, F), \quad 1 \leq q<\infty, \quad 0 \leq t<s<\infty,
$$

provided $E \hookrightarrow F$.

As usual,

$$
\stackrel{\circ}{W_{q}^{s}}(X, E):=\text { closure of } \mathcal{D}(X, E) \text { in } W_{q}^{s}(X, E) .
$$

Then

$$
\stackrel{\circ}{W_{q}^{s}}\left(\mathbb{R}^{n}, E\right)=W_{q}^{s}\left(\mathbb{R}^{n}, E\right), \quad s \in \mathbb{R}^{+}, \quad 1 \leq q<\infty .
$$

\section{$2.2 \quad$ Fourier Multipliers}

\subsubsection{Generalities}

Let $\mathcal{S}$ be the Schwartz space of rapidly decreasing functions on $\mathbb{R}^{n}$ and $\mathcal{S}^{\prime}$ its dual, the space of tempered distributions, endowed with the strong topology. We denote by $\mathcal{F}$ the Fourier transform $u \mapsto \hat{u}$ in $\mathcal{S}^{\prime}$, defined by $\langle\hat{u}, \phi\rangle=\langle u, \hat{\phi}\rangle$ and

$$
\hat{\phi}(\xi):=(2 \pi)^{-n / 2} \int_{\mathbb{R}^{n}} e^{-i<\xi, x>} \phi(x) d x, \quad \xi \in \mathbb{R}^{n}, \quad \phi \in \mathcal{S} .
$$

Recall that

$$
\mathcal{F} \in \mathcal{L} \text { aut }(\mathcal{S}) \cap \mathcal{L} \text { aut }\left(\mathcal{S}^{\prime}\right)
$$

and that

$$
\mathcal{F}^{-1} u=\check{\hat{u}}, \quad u \in \mathcal{S}^{\prime},
$$

where $\left\langle\check{u}, \phi>:=<u, \check{\phi}>\right.$ for $u \in \mathcal{S}^{\prime}$ and $\phi \in \mathcal{S}$ with $\check{\phi}(x):=\phi(-x)$ for $x \in \mathbb{R}^{n}$, and where $<\cdot, \cdot>:=<\cdot, \cdot\rangle_{\mathcal{S}}$.

Given $u \in \mathcal{S}^{\prime}$ and $\phi \in \mathcal{S}$, the convolution $u * \phi$ is a well defined tempered distribution and $(u * \phi)^{\wedge}=(2 \pi)^{n / 2} \hat{u} \hat{\phi}$ by the convolution theorem. Thus, given $a \in \mathcal{S}^{\prime}$, we define a linear operator, $a(D): \mathcal{S} \rightarrow \mathcal{S}^{\prime}$, a pseudodifferential operator with symbol $a$, by

$$
a(D) u:=\mathcal{F}^{-1} a \mathcal{F} u:=\mathcal{F}^{-1}(a \hat{u})=(2 \pi)^{-n / 2} \mathcal{F}^{-1} a * u, \quad u \in \mathcal{S} .
$$

It is well known that the convolution $u * v$ can be defined for various classes of distributions $u$ and $v$. Thus, given a Banach space with $E \hookrightarrow \mathcal{S}^{\prime}$, an element $a \in \mathcal{S}^{\prime}$ is said to be a Fourier multiplier for $E$ (an $E$-multiplier) if $\mathcal{F}^{-1} a * u$ is a well defined element of $E$ for each $u \in E$ and $\left(u \mapsto \mathcal{F}^{-1} a * u\right) \in \mathcal{L}(E)$. If this is the case, we again use the notations of (2.2.3). We denote by $M_{E}$ the vector space of all $E$-multipliers equipped with the norm

$$
\|a\|_{M_{E}}:=\|a(D)\|_{\mathcal{L}(E)} .
$$


In other words, $\|a\|_{M_{E}}$ is the infimum of all $c \in \mathbb{R}$ satisfying

$$
\left\|\mathcal{F}^{-1} a * u\right\|_{E} \leq c(2 \pi)^{n / 2}\|u\|_{E}, \quad u \in E .
$$

The notation $a(D)$ is, of course, justified by the well known formula

$$
\left(D^{\alpha} u\right)^{\wedge}=\xi^{\alpha} \hat{u}, \quad \alpha \in \mathbb{N}^{n}, \quad u \in \mathcal{S}^{\prime}
$$

Lastly, we recall the Plancherel's Theorem guarantees that

$$
\mathcal{F} \in \mathcal{L} \text { aut }\left(L_{2}\right)
$$

and

$$
\|\hat{u}\|_{2}=\|u\|_{2}, \quad u \in L_{2}
$$

\subsection{2 $L_{p}$-Multipliers}

We put $M_{p}:=M_{L_{p}}, 1 \leq p \leq \infty$. It is well known (e.g., [27]) that $M_{p}$ is a Banach algebra with respect to pointwise multiplication and that the map $a \mapsto a(D)$ from $M_{p}$ to $\mathcal{L}\left(L_{p}\right)$ is an algebra homomorphism. Moreover, (see Theorem 6.1.2 in [6])

$$
M_{1} \hookrightarrow M_{p} \hookrightarrow M_{q} \hookrightarrow M_{2}=L_{\infty}, \quad 1<p<q<2,
$$

and

$$
M_{p^{\prime}}=M_{p}, \quad 1 \leq p \leq 2, \frac{1}{p}+\frac{1}{p^{\prime}}=1
$$

Set

$$
\mathcal{M}_{0}:=\left(\left\{a \in L_{\infty}\left(\mathbb{R}^{n}\right) ;|\xi|^{|\alpha|} \partial^{\alpha} a \in L_{\infty}\left(\mathbb{R}^{n}\right),|\alpha| \leq[n / 2]+1\right\},\|\cdot\|_{\mathcal{M}_{0}}\right)
$$

where

$$
\|a\|_{\mathcal{M}_{0}}:=\max _{|\alpha| \leq\left[\frac{n}{2}\right]+1}\left\||\xi|^{|\alpha|} \partial^{\alpha} a\right\|_{\infty} .
$$

Then, by the Mikhlin's multiplier theorem (cf. Theorem 7.9.5 in [21] or Theorem 6.1.6 in [6]), we have the following theorem.

Theorem 2.2.1. $\mathcal{M}_{0} \hookrightarrow M_{p}, \quad 1<p<\infty$.

\subsection{3 $\quad L_{1}$-Multipliers}

It is well known that Theorem 2.2.1 is optimal in the sense that the elements of $\mathcal{M}_{0}$ are not $L_{1}$-multipliers, in general. The following corollary gives a simple sufficient condition for an element of $L_{\infty}$ to be an $L_{1}$-multiplier.

Consider the space

$$
\mathcal{F} L_{1}:=\left(\left\{u \in \mathcal{S}^{\prime} ; \hat{u} \in L_{1}\right\},\|u\|_{\mathcal{F} L_{1}}:=\|\hat{u}\|_{1}\right)
$$


and observe that, thanks to $(2.2 .1), \mathcal{F} L_{1}$ is a well defined Banach space. Also observe that, thanks to (2.2.1) and (2.2.2), it follows from Young's inequality for convolutions that

$$
\mathcal{F} L_{1} \hookrightarrow M_{1}
$$

Since there is no good direct description of $\mathcal{F} L_{1}$ available, we have to restrict our considerations to suitable subspaces of $\mathcal{F} L_{1}$ to obtain useful multiplier theorems for $L_{1}$.

Proposition 2.2.2. $W_{2}^{\left[\frac{n}{2}\right]+1}\left(\mathbb{R}^{n}\right) \hookrightarrow \mathcal{F} L_{1}$.

Proof It follows from (2.1.15) and Plancherel's theorem that, given $m \in \dot{\mathbb{N}}$,

$$
\|u\|_{m, 2}=\left(\sum_{|\alpha| \leq m}\left\|\xi^{\alpha} \hat{u}\right\|_{2}^{2}\right)^{1 / 2}, \quad u \in W_{2}^{m}\left(\mathbb{R}^{n}\right) .
$$

Let

$$
\Lambda_{1}(\xi):=\left(1+|\xi|^{2}\right)^{1 / 2}, \quad \xi \in \mathbb{R}^{n},
$$

and observe that (2.2.11) and the multinomial theorem imply

$$
\|\cdot\|_{m, 2} \sim\left\|\Lambda_{1}^{m} \mathcal{F} \cdot\right\|_{2}
$$

where $\sim$ denotes equivalent norms. By the Cauchy-Schwarz inequality

$$
\|u\|_{\mathcal{F} L_{1}}=\|\hat{u}\|_{1}=\left\|\Lambda_{1}^{m} \hat{u} \Lambda_{1}^{-m}\right\|_{1} \leq\left\|\Lambda_{1}^{m} \hat{u}\right\|_{2}\left\|\Lambda_{1}^{-m}\right\|_{2},
$$

and $\Lambda_{1}^{-m} \in L_{2}$ iff $m>n / 2$. Thus the assertion is a consequence of (2.2.13) and (2.2.14).

Corollary 2.2.3. $W_{2}^{\left[\frac{n}{2}\right]+1}\left(\mathbb{R}^{n}\right) \hookrightarrow M_{q}, \quad 1 \leq q \leq \infty$.

Proof Recall (2.2.7), (2.2.8) and (2.2.10), this is an immediate consequence of Proposition 2.2.2.

Unfortunately, the space $W_{2}^{\left[\frac{n}{2}\right]+1}\left(\mathbb{R}^{n}\right)$ is too small to be a useful space of multipliers for $L_{1}$ and $L_{\infty}$. For this reason we introduce now a Banach space $\mathcal{M}$ - a subspace of $\mathcal{M}_{0}$ - which will turn out to be a fundamental space of multipliers for our purposes.

Set

$$
\mathcal{M}:=\left(\left\{a \in L_{\infty}\left(\mathbb{R}^{n}\right) ; \Lambda_{1}^{|\alpha|} \partial^{\alpha} a \in L_{\infty}\left(\mathbb{R}^{n}\right),|\alpha| \leq[n / 2]+1\right\},\|\cdot\|_{\mathcal{M}}\right),
$$

where

$$
\|a\|_{\mathcal{M}}:=\max _{|\alpha| \leq\left[\frac{n}{2}\right]+1}\left\|\Lambda_{1}^{|\alpha|} \partial^{\alpha} a\right\|_{\infty},
$$

and $\Lambda_{1}$ is defined in (2.2.12). 


\section{$2.3 \quad$ Besov Spaces}

\subsubsection{A Partition of Unity}

Let $\psi \in \mathcal{D}:=\mathcal{D}\left(\mathbb{R}^{n}, \mathbb{C}\right)$ be fixed with $\operatorname{supp}(\psi) \subset(3 / 2) \mathbb{B}$, where $\mathbb{B}:=\mathbb{B}_{\mathbb{R}^{n}}$, and such that $0 \leq \psi \leq 1$ and $\psi \mid \mathbb{B}=1$. Put

$$
\varphi_{0}(x):=\psi(x), \quad \varphi_{1}(x):=\psi(x / 2)-\psi(x), \quad \varphi_{k}(x):=\varphi_{1}\left(2^{-k+1} x\right)
$$

for $x \in \mathbb{R}^{n}$ and $k \in \dot{\mathbb{N}}$. Then

$$
\begin{aligned}
& \sum_{j=0}^{m} \varphi_{j}(x)=\psi\left(2^{-m} x\right), \quad m \in \mathbb{N}, \\
& \sum_{j=0}^{\infty} \varphi_{j}(x)=1, \quad x \in \mathbb{R}^{n}, \\
& \operatorname{supp}\left(\varphi_{j}\right) \subset\left\{x \in \mathbb{R}^{n} ; 2^{j-1} \leq|x| \leq 3 \cdot 2^{j-1}\right\}, \quad j \in \dot{\mathbb{N}},
\end{aligned}
$$

and

$$
\varphi_{j}(x)=1, \quad 3 \cdot 2^{j-2} \leq|x| \leq 2^{j}, \quad j \in \dot{\mathbb{N}} .
$$

\subsubsection{Definition of Besov Spaces}

Given $s \in \mathbb{R}$ and $p, q \in[1, \infty]$, the Besov space, $B_{p, q}^{s}$ of order $s$ (and integrability orders $p$ and $q$ ) is defined by

$$
B_{p, q}^{s}:=B_{p, q}^{s}\left(\mathbb{R}^{n}, \mathbb{C}\right):=\left(\left\{u \in \mathcal{S}^{\prime} ;\|u\|_{B_{p, q}^{s}}<\infty\right\},\|\cdot\|_{B_{p, q}^{s}}\right),
$$

where

$$
\|u\|_{B_{p, q}^{s}}:=\left\|2^{s k} \varphi_{k}(D) u\right\|_{l_{q}\left(L_{p}\right)}=\left(\sum_{k=0}^{\infty}\left(\int_{\mathbb{R}^{n}}\left|2^{s k}(2 \pi)^{-n / 2} \mathcal{F}^{-1} \varphi_{k} * u(x)\right|^{p} d x\right)^{q / p}\right)^{1 / q}
$$

and where $\left(\varphi_{k}\right)$ is the partition of unity of Subsection 2.3.1. It is well known that these Besov spaces are Banach spaces which - except for equivalent norms — are independent of the choice of $\psi$.

\subsubsection{The Mikhlin-Hörmander theorem on Besov spaces}

It follows from the definition of the Besov spaces and known properties of spaces of distributions that

$$
\mathcal{D} \stackrel{d}{\hookrightarrow} \mathcal{S} \hookrightarrow B_{p, 1}^{s} \hookrightarrow B_{p, q}^{s} \hookrightarrow B_{p, \infty}^{s} \hookrightarrow B_{p, 1}^{t} \stackrel{d}{\hookrightarrow} \mathcal{S}^{\prime} \stackrel{d}{\hookrightarrow} \mathcal{D}^{\prime}, \quad s>t
$$

and that

$$
\mathcal{S} \stackrel{d}{\hookrightarrow} B_{p, q}^{s}, \quad p \vee q<\infty
$$


where $p \vee q:=\max \{p, q\}$. In particular,

$$
B_{\infty, \infty}^{s} \doteq B U C^{s}, \quad s \in \mathbb{R}^{+} \backslash \mathbb{N}
$$

where the symbol $\doteq$ denote equivalent spaces $($ cf. Proposition 6 in Section V.4.1 in [27]).

It is well known that

$$
M_{B_{p, q}^{0}}=M_{B_{p, q}^{s}}, \quad p, q \in[1, \infty], s \in \mathbb{R} .
$$

In order to prove Mikhlin-Hörmander theorem we need the following simple and well known facts about dilatations.

Given $t>0$, define the dilatation, $\sigma_{t}$, by

$$
\sigma_{t} \varphi(x):=\varphi(t x), \quad x \in \mathbb{R}^{n}, \quad \varphi \in \mathcal{S},
$$

and by

$$
<\sigma_{t} u, \varphi>:=t^{-n}\left\langle u, \sigma_{1 / t} \varphi>, \quad u \in \mathcal{S}^{\prime}, \quad \varphi \in \mathcal{S} .\right.
$$

Then

$$
\left\{\sigma_{t} ; t>0\right\} \text { is a subgroup of } \mathcal{L} \text { aut }(\mathcal{S}) \text { and of } \mathcal{L} \text { aut }\left(\mathcal{S}^{\prime}\right)
$$

and

$$
\left(\sigma_{t}\right)^{-1}=\sigma_{1 / t}
$$

Furthermore,

$$
\partial^{\alpha} \sigma_{t}=t^{|\alpha|} \sigma_{t} \partial^{\alpha}, \quad \alpha \in \mathbb{N}^{n}
$$

and

$$
\mathcal{F} \sigma_{t}=t^{-n} \sigma_{1 / t} \mathcal{F}
$$

Lastly,

$$
\left\|\sigma_{t} u\right\|_{p}=t^{-n / p}\|u\|_{p}, \quad 1 \leq p \leq \infty .
$$

Theorem 2.3.1 (Mikhlin-Hörmander Theorem).

$$
\mathcal{M} \hookrightarrow M_{B_{p, q}^{s}}, \quad p, q \in[1, \infty], \quad s \in \mathbb{R} .
$$

Proof $\quad$ Fix $\chi_{j} \in \mathcal{D}, j=0,1$, such that $\operatorname{supp}\left(\chi_{0}\right) \subset 2 \mathbb{B}$ and $\chi_{0} \mid(3 / 2) \mathbb{B}=1$, and such that $\operatorname{supp}\left(\chi_{1}\right) \subset 4 \mathbb{B} \backslash(1 / 2) \mathbb{B}$ and $\chi_{1} \mid(3 \mathbb{B} \backslash \mathbb{B})=1$. Moreover, put

$$
\chi_{k}:=\sigma_{2^{-k+1}} \chi_{1}, \quad k \in \dot{\mathbb{N}} .
$$

Given $a \in \mathcal{M}$, since $\varphi_{k} \chi_{k}=\varphi_{k}$ for $k \in \mathbb{N}$, then

$$
\begin{aligned}
\varphi_{k}(D) a(D) u & =\mathcal{F}^{-1} \varphi_{k} \mathcal{F} \mathcal{F}^{-1} a \mathcal{F} u=\mathcal{F}^{-1} \varphi_{k} a \mathcal{F} u=\mathcal{F}^{-1} a \chi_{k} \varphi_{k} \mathcal{F} u \\
& =\mathcal{F}^{-1} \chi_{k} a \mathcal{F} \mathcal{F}^{-1} \varphi_{k} \mathcal{F} u=\mathcal{F}^{-1} \chi_{k} a \mathcal{F} \varphi_{k}(D) u, \quad u \in \mathcal{S}^{\prime}
\end{aligned}
$$


where $\varphi_{k}$ has been defined in (2.3.1). Since $\chi_{k} a=\left(\sigma_{2^{-k+1}} \chi_{1}\right) a=\sigma_{2^{-k+1}}\left(\chi_{1} \sigma_{2^{k-1}} a\right)$ for $k \in \dot{\mathbb{N}}$, it follows from $(2.3 .11),(2.3 .9)$ and $(2.2 .2)$ that

$$
\begin{aligned}
\varphi_{k}(D) a(D) u & =\mathcal{F}^{-1}\left\{\sigma_{2^{-k+1}}\left(\chi_{1} \sigma_{2^{k-1}} a\right)\right\} \mathcal{F} \varphi_{k}(D) u \\
& =\mathcal{F}^{-1} \sigma_{2^{-k+1}}\left\{\chi_{1}\left(\sigma_{2^{k-1}} a\right)\left(\sigma_{2^{k-1}} \mathcal{F} \varphi_{k}(D) u\right)\right\} \\
& =2^{(k-1) n} \sigma_{2^{k-1}}\left\{\mathcal{F}^{-1}\left(\chi_{1} a_{k}\left(2^{(1-k) n} \mathcal{F}\left(\sigma_{2^{-k+1}}\left(\varphi_{k}(D) u\right)\right)\right)\right\}\right. \\
& =\sigma_{2^{k-1}} \mathcal{F}^{-1} \chi_{1} a_{k} \mathcal{F} \sigma_{2^{-k+1}}\left(\varphi_{k}(D) u\right) \\
& =\sigma_{2^{k-1}}\left(\chi_{1} a_{k}\right)(D) \sigma_{2^{-k+1}}\left(\varphi_{k}(D) u\right)
\end{aligned}
$$

for $k \in \dot{\mathbb{N}}$, where

$$
a_{k}:=\sigma_{2^{k-1}} a .
$$

Observe that $\chi_{0}, \chi_{1} \in \mathcal{D}$ and $a \in \mathcal{M}$, then

$$
\chi_{0} a, \chi_{1} a_{k} \in W_{2}^{m}, \quad k \in \dot{\mathbb{N}},
$$

where $m:=[n / 2]+1$. Thus we deduce from (2.3.12), Corollary 2.2.3 and (2.3.10) that

$$
\begin{aligned}
\left\|\varphi_{k}(D) a(D) u\right\|_{p} & =2^{(1-k) n / p}\left\|\left(\chi_{1} a_{k}\right)(D) \sigma_{2^{-k+1}}\left(\varphi_{k}(D) u\right)\right\|_{p} \\
& \leq c 2^{(1-k) n / p}\left\|\chi_{1} a_{k}\right\|_{m, 2}\left\|\sigma_{2^{-k+1}}\left(\varphi_{k}(D) u\right)\right\|_{p} \\
& =c\left\|\chi_{1} a_{k}\right\|_{m, 2}\left\|\varphi_{k}(D) u\right\|_{p}
\end{aligned}
$$

for $1 \leq p \leq \infty$ and $k \in \dot{\mathbb{N}}$. Similarly,

$$
\left\|\varphi_{0}(D) a(D) u\right\|_{p} \leq c\left\|\chi_{0} a\right\|_{m, 2}\left\|\varphi_{0}(D) u\right\|_{p}, \quad 1 \leq p \leq \infty .
$$

It follows from (2.3.8) that

$$
\partial^{\alpha} a_{k}=\partial^{\alpha}\left(\sigma_{2^{k-1}} a\right)=2^{(k-1)|\alpha|} \sigma_{2^{k-1}}\left(\partial^{\alpha} a\right), \quad \alpha \in \mathbb{N}^{n} .
$$

Thus $a \in \mathcal{M}$ implies

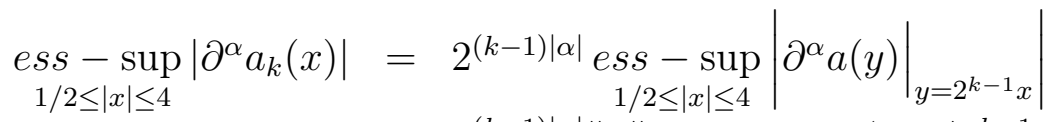

$$
\begin{aligned}
& \leq 2^{(k-1)|\alpha|}\|a\|_{\mathcal{M}} \text { ess }-\sup \left(1+\left|2^{k-1} x\right|^{2}\right)^{-|\alpha| / 2} \\
& \leq 2^{(k-1)|\alpha|}\|a\|_{\mathcal{M}} 2^{-(k-2)|\alpha|} \\
& =2^{|\alpha|}\|a\|_{\mathcal{M}}
\end{aligned}
$$

for $|\alpha| \leq m$ and $k \in \dot{\mathbb{N}}$. By means of these estimates, Leibniz' rule, and (2.3.13), (2.3.14), we deduce that

$$
\left\|\varphi_{k}(D) a(D) u\right\|_{p} \leq c\|a\|_{\mathcal{M}}\left\|\varphi_{k}(D) u\right\|_{p}, \quad 1 \leq p \leq \infty, \quad k \in \mathbb{N} .
$$

Now it follows from the definition of the norm of $B_{p, q}^{0}$ that

$$
\|a(D) u\|_{B_{p, q}^{0}} \leq c\|a\|_{\mathcal{M}}\|u\|_{B_{p, q}^{0}}, \quad 1 \leq p, q \leq \infty, \quad u \in B_{p, q}^{0} .
$$

This means $\mathcal{M} \hookrightarrow M_{B_{p, q}^{0}}$. Thanks to (2.3.4), we get that

$$
\mathcal{M} \hookrightarrow M_{B_{p, q}^{s}}, \quad p, q \in[1, \infty], \quad s \in \mathbb{R} .
$$

Corollary 2.3.2. $\mathcal{M} \hookrightarrow M_{B U C^{s}}, \quad s \in \mathbb{R}^{+} \backslash \mathbb{N}$.

Proof This is a direct consequence of (2.3.3) and Theorem 2.3.1. 


\subsection{Interpolation Theory}

In this section we collect some facts on interpolation theory. Our representation follows closely the monograph [24] of Lunardi. If $X, Y, D$ are Banach spaces such that

$$
D \hookrightarrow Y \hookrightarrow X
$$

we say that $Y$ is an intermediate space between $X$ and $D$. If, in addition, for every linear operator $T \in \mathcal{L}(X)$ such that $\left.T\right|_{D} \in \mathcal{L}(D)$ it holds $\left.T\right|_{Y} \in \mathcal{L}(Y)$, then $Y$ is called interpolation space between $X$ and $D$.

\subsubsection{Interpolatory Inclusion}

Let $X, D$ be Banach space, with norms $\|\cdot\|,\|\cdot\|_{D}$ respectively, and assume that $D$ is continuously embedded in $X$.

Definition 2.4.1. Let $0 \leq \alpha \leq 1$. A Banach space $Y$ such that $D \hookrightarrow Y \hookrightarrow X$ is said to belong to the class $J_{\alpha}$ between $X$ and $D$ if there is a constant $c>0$ such that

$$
\|x\|_{Y} \leq c\|x\|^{1-\alpha}\|x\|_{D}^{\alpha}, \quad \forall x \in D .
$$

In this case we write $Y \in J_{\alpha}(X, D)$.

Some important examples are given in the next propositions.

Proposition 2.4.2. Let $k, m$ be positive integers such that $k<m$. Then:

(i) $B C^{k}(\mathbb{R}, X)$ belongs to the class $J_{k / m}$ between $B C(\mathbb{R}, X)$ and $B C^{m}(\mathbb{R}, X)$;

(ii) $B C^{k}\left(\mathbb{R}^{n}\right)$ belongs to the class $J_{k / m}$ between $B C\left(\mathbb{R}^{n}\right)$ and $B C^{m}\left(\mathbb{R}^{n}\right) ; B C^{k}\left(\mathbb{R}_{+}^{n}\right)$ belongs to the class $J_{k / m}$ between $B C\left(\mathbb{R}_{+}^{n}\right)$ and $B C^{m}\left(\mathbb{R}_{+}^{n}\right)$;

(iii) if $\Omega$ is an open set in $\mathbb{R}^{n}$ with uniformly $C^{m}$ boundary, then $B C^{k}(\bar{\Omega})$ belongs to the class $J_{k / m}$ between $B C(\bar{\Omega})$ and $B C^{m}(\bar{\Omega})$.

Proof Let us prove statement (i). It is sufficient to show that for every $m \in \mathbb{N}$ there is $c_{m}>0$ such that if $B C^{m}(\mathbb{R}, X)$ then

$$
\left\|f^{(k)}\right\|_{\infty} \leq c_{m}\left(\|f\|_{\infty}\right)^{1-k / m}\left(\left\|f^{(m)}\right\|_{\infty}\right)^{k / m}, \quad \forall k=1, \ldots, m-1 .
$$

First we prove that (2.4.1) holds for $m=2$ and $k=1$. From the inequality

$$
\left\|f(x+h)-f(x)-f^{\prime}(x) h\right\| \leq \frac{1}{2}\left\|f^{\prime \prime}\right\|_{\infty} h^{2}, \quad \forall x \in \mathbb{R}, h>0,
$$

we get

$$
\left\|f^{\prime}(x)\right\| \leq \frac{\|f(x+h)-f(x)\|}{h}+\frac{1}{2}\left\|f^{\prime \prime}\right\|_{\infty} h, \quad \forall x \in \mathbb{R}, h>0,
$$


so that

$$
\left\|f^{\prime}\right\|_{\infty} \leq \frac{2\|f\|_{\infty}}{h}+\frac{1}{2}\left\|f^{\prime \prime}\right\|_{\infty} h, \quad \forall h>0 .
$$

Taking the minimum on $h$ over $(0,+\infty)$ we get

$$
\left\|f^{\prime}\right\|_{\infty} \leq 2\left(\|f\|_{\infty}\right)^{1 / 2}\left(\left\|f^{\prime \prime}\right\|_{\infty}\right)^{1 / 2}, \quad \forall f \in B C^{2}(\mathbb{R}, X) .
$$

Assume now by recurrence that (2.4.1) holds for some $m>2$. Then for every $f \in$ $B C^{m+1}(\mathbb{R}, X)$ we get

$$
\begin{aligned}
\left\|f^{\prime}\right\|_{\infty} & \leq c_{m}\left(\|f\|_{\infty}\right)^{1-1 / m}\left(\left\|f^{(m)}\right\|_{\infty}\right)^{1 / m} \\
& \leq c_{m}\left(\|f\|_{\infty}\right)^{1-1 / m}\left[c_{m}\left(\left\|f^{\prime}\right\|_{\infty}\right)^{1-\frac{m-1}{m}}\left(\left\|f^{(m+1)}\right\|_{\infty}\right)^{\frac{m-1}{m}}\right]^{1 / m} .
\end{aligned}
$$

It follows that

$$
\left\|f^{\prime}\right\|_{\infty} \leq c_{m}^{\frac{m+1}{m}}\left(\|f\|_{\infty}\right)^{1-\frac{1}{m+1}}\left(\left\|f^{(m+1)}\right\|_{\infty}\right)^{\frac{1}{m+1}}
$$

and , for $k=2, \ldots, m$,

$$
\begin{aligned}
\left\|f^{(k)}\right\|_{\infty}=\left\|\left(f^{\prime}\right)^{(k-1)}\right\|_{\infty} & \leq c_{m}\left(\left\|f^{\prime}\right\|_{\infty}\right)^{1-(k-1) / m}\left(\left\|f^{(m+1)}\right\|_{\infty}\right)^{(k-1) / m} \\
& \leq c_{m}^{1+\frac{m-k+1}{m-1}}\left(\|f\|_{\infty}\right)^{1-\frac{k}{m+1}}\left(\left\|f^{(m+1)}\right\|_{\infty}\right)^{\frac{k}{m+1}}
\end{aligned}
$$

Therefore, (2.4.1) holds with $m$ replaced by $m+1$, and statement (i) is proved.

Statement (ii) can be shown similarly, replacing $h$ by $h e_{i}$, where $e_{i}$ is the vector whose $k$-th component is 0 if $k \neq i, 1$ if $k=i$.

Statement (iii) follows from (ii) by localizing and straightening the boundary.

Similar arguments lead to the following generalization.

Proposition 2.4.3. Let $0<\theta<\alpha$. Then:

(i) $B U C^{\theta}(\mathbb{R}, X)$ belongs to the class $J_{\theta / \alpha}$ between $B C(\mathbb{R}, X)$ and $B U C^{\alpha}(\mathbb{R}, X)$;

(ii) $B U C^{\theta}\left(\mathbb{R}^{n}\right)$ belongs to the class $J_{\theta / \alpha}$ between $B C\left(\mathbb{R}^{n}\right)$ and $B U C^{\alpha}\left(\mathbb{R}^{n}\right) ; B U C^{\theta}\left(\mathbb{R}_{+}^{n}\right)$ belongs to the class $J_{\theta / \alpha}$ between $B C\left(\mathbb{R}_{+}^{n}\right)$ and $B U C^{\alpha}\left(\mathbb{R}_{+}^{n}\right)$;

(iii) if $\Omega$ is an open set in $\mathbb{R}^{n}$ with uniformly $C^{\alpha}$ boundary, then $B U C^{\theta}(\bar{\Omega})$ belongs to the class $J_{\theta / \alpha}$ between $B C(\bar{\Omega})$ and $B U C^{\alpha}(\bar{\Omega})$.

Now we are able to state some interpolatory inclusions between spaces of functions defined in an interval $I$.

Proposition 2.4.4. Let $X_{\alpha} \in J_{\alpha}(X, D)$. Then

(i) $B(I, D) \cap \operatorname{Lip}(I, X) \hookrightarrow B U C^{1-\alpha}\left(I, X_{\alpha}\right)$,

(ii) $B(I, D) \cap B U C^{\theta}(I, X) \hookrightarrow B U C^{\theta(1-\alpha)}\left(I, X_{\alpha}\right), \quad \forall \theta \in(0,1)$,

(iii) $B(I, D) \cap B C(I, X) \hookrightarrow B C\left(I, X_{\alpha}\right)$. 
Proof Let us prove statement (i). For every $u \in B(I, D) \cap \operatorname{Lip}(I, X)$ and for $s, t \in I$ we have

$$
\begin{aligned}
\|u(t)-u(s)\|_{X_{\alpha}} & \leq c\|u(t)-u(s)\|^{1-\alpha}\|u(t)-u(s)\|_{D}^{\alpha} \\
& \leq c[u]_{L i p(I, X)}^{1-\alpha}|t-s|^{1-\alpha}\left(2\|u\|_{B(I, D)}\right)^{\alpha}
\end{aligned}
$$

so that $u$ is uniformly $(1-\alpha)$-Hölder continuous with values in $X_{\alpha}$, and

$$
\begin{aligned}
\|u\|_{B U C^{1-\alpha}\left(I, X_{\alpha}\right)} & \leq c\|u\|_{B(I, X)}^{1-\alpha}\|u\|_{B(I, D)}^{\alpha}+2^{\alpha} c[u]_{L i p(I, X)}^{1-\alpha}\|u\|_{B(I, D)}^{\alpha}, \\
& \leq\left(2^{\alpha}+1\right) c\|u\|_{L i p(I, X)}^{1-\alpha}\|u\|_{B(I, D)}^{\alpha} .
\end{aligned}
$$

The proof of statements (ii) and (iii) is similar.

\subsubsection{The $K$-method}

Let $X, Y$ be Banach spaces, with $Y \hookrightarrow X$, and let $c>0$ be such that

$$
\|y\|_{X} \leq c\|y\|_{Y}, \quad \forall y \in Y .
$$

We describe briefly the construction of a family of intermediate spaces between $X$ and $Y$, called real interpolation spaces, and denoted by $(X, Y)_{\theta, p},(X, Y)_{\theta}$, with $0<\theta \leq 1,1 \leq p \leq \infty$, see also [6, 29]. We follow the so-called $K$-method, and we always set $1 / \infty=0$.

Definition 2.4.5. For every $x \in X$ and $t>0$, set

$$
K(t, x, X, Y)=\inf _{x=a+b, a \in X, b \in Y}\left(\|a\|_{X}+t\|b\|_{Y}\right) .
$$

If there is no danger of confusion, we shall write $K(t, x)$ instead of $K(t, x, X, Y)$.

From Definition 2.4.5 it follows immediately that for every $t>0$ and $x \in X$ we have

$$
\left\{\begin{array}{l}
\text { (i) } \quad \min \{1, t\} K(1, x) \leq K(t, x) \leq \max \{1, t\} K(1, x), \\
\text { (ii) } \quad K(t, x) \leq\|x\|_{X} .
\end{array}\right.
$$

Now we define a family of Banach spaces by means of the function $K$.

Definition 2.4.6. Let $0<\theta \leq 1,1 \leq p \leq \infty$, and set

$$
\begin{aligned}
& \left\{\begin{array}{l}
(X, Y)_{\theta, p}=\left\{x \in X ; t \mapsto t^{-\theta-1 / p} K(t, x, X, Y) \in L_{p}(0,+\infty)\right\} \\
\|x\|_{(X, Y)_{\theta, p}}=\left\|t^{-\theta-1 / p} K(t, x, X, Y)\right\|_{L_{p}(0,+\infty)}
\end{array}\right. \\
& (X, Y)_{\theta}=\left\{x \in X ; \lim _{t \rightarrow 0} t^{-\theta} K(t, x, X, Y)=0\right\} .
\end{aligned}
$$


The mapping $x \mapsto\|x\|_{(X, Y)_{\theta, p}}$ is easily seen to be a norm in $(X, Y)_{\theta, p}$. Where there is no danger of confusion, we shall write $\|x\|_{\theta, p}$ instead of $\|x\|_{(X, Y)_{\theta, p}}$.

Since $t \mapsto K(t, x)$ is bounded, it is clear that only the behavior near $t=0$ of $t^{-\theta} K(t, x)$ plays a role in the definition of $(X, Y)_{\theta, p}$ and of $(X, Y)_{\theta}$.

For $\theta=1$, from the first inequality in $(2.4 .4)(\mathrm{i})$ we get

$$
(X, Y)_{1}=(X, Y)_{1, p}=\{0\}, \quad p<\infty .
$$

Therefore, from now on we shall consider the case $(\theta, p) \in(0,1) \times[1,+\infty]$ and $(\theta, p)=$ $(1, \infty)$.

If $X=Y$, then $K(t, x)=\min \{t, 1\}\|x\|$. Therefore, as one can expect, $(X, X)_{\theta, p}=$ $(X, X)_{1, \infty}=X$ for $0<\theta<1,1 \leq p \leq \infty$, and

$$
\begin{array}{r}
\|x\|_{(X, X)_{\theta, p}}=\left(\frac{1}{p \theta(1-\theta)}\right)^{1 / p}\|x\|_{X}, \quad 0<\theta<1, p<\infty, \\
\|x\|_{(X, X)_{\theta, \infty}}=\|x\|_{X}, \quad 0<\theta \leq 1 .
\end{array}
$$

Some inclusion properties are stated below.

Proposition 2.4.7. For $0<\theta<1,1 \leq p_{1} \leq p_{2} \leq \infty$ we have

$$
Y \subset(X, Y)_{\theta, p_{1}} \subset(X, Y)_{\theta, p_{2}} \subset(X, Y)_{\theta} \subset(X, Y)_{\theta, \infty} \subset \bar{Y} .
$$

For $0<\theta_{1}<\theta_{2} \leq 1$ we have

$$
(X, Y)_{\theta_{2}, \infty} \subset(X, Y)_{\theta_{1}, 1} .
$$

Proof From the inequality $K(t, x) \leq \min \{c, t\}\|x\|_{Y}$ for every $x \in Y$, it follows immediately that $Y$ is continuously embedded in $(X, Y)_{1, \infty}$ and in $(X, Y)_{\theta, p}$ for $0<\theta<1$, $1 \leq p \leq \infty$.

Let us show that $(X, Y)_{\theta, \infty}$ is contained in $\bar{Y}$ and it is continuously embedded in $X$. For $x \in(X, Y)_{\theta, \infty}$ and for every $n \in \mathbb{N}$ there are $a_{n} \in X, b_{n} \in Y$ such that $x=a_{n}+b_{n}$, and

$$
n^{\theta}\left(\left\|a_{n}\right\|_{X}+\frac{1}{n}\left\|b_{n}\right\|_{Y}\right) \leq 2\|x\|_{\theta, \infty} .
$$

In particular, $\left\|x-b_{n}\right\|_{X}=\left\|a_{n}\right\|_{X} \leq 2\|x\|_{\theta, \infty} n^{-\theta}$, so that the sequence $\left\{b_{n}\right\}$ goes to $x$ in $X$ as $n \rightarrow \infty$. This implies that $(X, Y)_{\theta, \infty}$ is contained in $\bar{Y}$. Moreover, from the inequality

$$
\|x\|_{X} \leq\|a\|_{X}+\|b\|_{X} \leq\|a\|_{X}+c\|b\|_{Y}, \quad \text { if } \quad x=a+b,
$$

we get

$$
\|x\|_{X} \leq K(c, x) \leq c^{\theta}\|x\|_{\theta, \infty}, \quad \forall x \in(X, Y)_{\theta, \infty},
$$

so that $(X, Y)_{\theta, \infty}$ is continuously embedded in $X$.

The inclusion $(X, Y)_{\theta} \subset(X, Y)_{\theta, \infty}$ is trivial, since $K(\cdot, x)$ is bounded. 
Let us show that $(X, Y)_{\theta, p}$ is contained in $(X, Y)_{\theta}$ and it is continuously embedded in $(X, Y)_{\theta, \infty}$ for $p<\infty$. Note that $K(\cdot, x)$ satisfies

$$
K(t, x) \leq \frac{t}{s} K(s, x) \quad \text { for } x \in X, 0<s<t
$$

Therefore, for each $x \in(X, Y)_{\theta, p}$ and $t>0$,

$$
\begin{aligned}
t^{1-\theta} K(t, x) & =[(1-\theta) p]^{1 / p}\left(\int_{0}^{t} s^{(1-\theta) p-1} d s\right)^{1 / p} K(t, x) \\
& \leq[(1-\theta) p]^{1 / p}\left(\int_{0}^{t} s^{-\theta p-1} t^{p} K(s, x)^{p} d s\right)^{1 / p}
\end{aligned}
$$

so that

$$
t^{-\theta} K(t, x) \leq[(1-\theta) p]^{1 / p}\left(\int_{0}^{t} s^{-\theta p-1} K(s, x)^{p} d s\right)^{1 / p} .
$$

Letting $t \rightarrow 0$, it follows that $x \in(X, Y)_{\theta}$. The same inequality yields

$$
\|x\|_{\theta, \infty} \leq[(1-\theta) p]^{1 / p}\|x\|_{\theta, p} .
$$

Let us prove that $(X, Y)_{\theta, p_{1}} \subset(X, Y)_{\theta, p_{2}}$ for $p_{1}<p_{2}$. For $x \in(X, Y)_{\theta, p_{1}}$ we have

$$
\begin{aligned}
& \|x\|_{\theta, p_{2}}=\left(\int_{0}^{+\infty} t^{-\theta p_{2}-1} K(t, x)^{p_{2}} d t\right)^{1 / p_{2}} \\
& \leq\left(\int_{0}^{+\infty} t^{-\theta p_{1}-1} K(t, x)^{p_{1}} d t\right)^{1 / p_{2}}\left(\sup _{t>0} t^{-\theta} K(t, x)\right)^{\left(p_{2}-p_{1}\right) / p_{2}} \\
& =\left(\|x\|_{\theta, p_{1}}\right)^{p_{1} / p_{2}}\left(\|x\|_{\theta, \infty}\right)^{1-p_{1} / p_{2}},
\end{aligned}
$$

and using (2.4.9) we find

$$
\|x\|_{\theta, p_{2}} \leq\left[(1-\theta) p_{1}\right]^{1 / p_{1}-1 / p_{2}}\|x\|_{\theta, p_{1}} .
$$

Let us prove that (2.4.8) holds. If $0<\theta_{1}<\theta_{2} \leq 1$ and $x \in(X, Y)_{\theta_{2}, \infty}$, we have

$$
\begin{aligned}
\|x\|_{\theta_{1}, 1} & =\int_{0}^{1} t^{-\theta_{1}-1} K(t, x) d t+\int_{1}^{+\infty} t^{-\theta_{1}-1} K(t, x) d t \\
& \leq \int_{0}^{1} t^{-\theta_{1}-1}\|x\|_{\theta_{2}, \infty} t^{\theta_{2}} d t+\int_{1}^{+\infty} t^{-\theta_{1}-1}\|x\|_{X} d t \\
& \leq \frac{1}{\theta_{2}-\theta_{1}}\|x\|_{\theta_{2}, \infty}+\frac{1}{\theta_{1}}\|x\|_{X} .
\end{aligned}
$$

The statement is so completely proved.

Proposition 2.4.8. $(X, Y)_{\theta, p}$ is a Banach space. 
Proof Let $\left\{x_{n}\right\}_{n \in \mathbb{N}}$ be a Cauchy sequence in $(X, Y)_{\theta, p}$. Due to the continuous embedding of $(X, Y)_{\theta, p}$ in $X,\left\{x_{n}\right\}_{n \in \mathbb{N}}$ is a Cauchy sequence in $X$ too, so that it converges to an element $x \in X$.

Let us estimate $\left\|x_{n}-x\right\|_{\theta, p}$. Fix $\varepsilon>0$, and let $\left\|x_{n}-x_{m}\right\|_{\theta, p} \leq \varepsilon$ for $n, m \geq n_{\varepsilon}$. Since $y \mapsto K(t, y)$ is a norm, for every $n, m \in \mathbb{N}$ and $t>0$ we have

$$
t^{-\theta} K\left(t, x_{n}-x\right) \leq t^{-\theta} K\left(t, x_{n}-x_{m}\right)+t^{-\theta}\left\|x_{m}-x\right\|_{X} .
$$

Let $p=\infty$. Then for every $t>0$ and $n, m \geq n_{\varepsilon}$

$$
t^{-\theta} K\left(t, x_{n}-x\right) \leq \varepsilon+t^{-\theta}\left\|x_{m}-x\right\|_{X}
$$

Letting $m \rightarrow+\infty$, we find $t^{-\theta} K\left(t, x_{n}-x\right) \leq \varepsilon$ for every $t>0$. This implies that $x \in(X, Y)_{\theta, \infty}$ and that $x_{n} \rightarrow x$ in $(X, Y)_{\theta, \infty}$.

Let now $p<\infty$. Then

$$
\left\|x_{n}-x\right\|_{\theta, p}=\lim _{\delta \rightarrow 0}\left(\int_{\delta}^{1 / \delta} t^{-\theta p-1} K\left(t, x_{n}-x\right)^{p} d t\right)^{1 / p}
$$

Due again to $(2.4 .12)$, for every $\delta \in(0,1)$ we get, for $n, m \geq n_{\varepsilon}$,

$$
\begin{aligned}
& \left(\int_{\delta}^{1 / \delta} t^{-\theta p-1} K\left(t, x_{n}-x\right)^{p} d t\right)^{1 / p} \\
\leq & \left\|x_{n}-x_{m}\right\|_{\theta, p}+\left\|x_{m}-x\right\|_{X}\left(\int_{\delta}^{1 / \delta} t^{-\theta p-1} d t\right)^{1 / p} \\
\leq & \varepsilon+\left\|x_{m}-x\right\|_{X}\left(\frac{1}{\theta p \delta^{\theta p}}\right)^{1 / p} .
\end{aligned}
$$

Letting $m \rightarrow \infty$ and then $\delta \rightarrow 0$ we get $x \in(X, Y)_{\theta, p}$ and $x_{n} \rightarrow x$ in $(X, Y)_{\theta, p}$.

Corollary 2.4.9. For $0<\theta \leq 1,(X, Y)_{\theta}$ is a Banach space, endowed with the norm of $(X, Y)_{\theta, \infty}$.

Proof It is easy to see that $(X, Y)_{\theta}$ is a closed subspace of $(X, Y)_{\theta, \infty}$. By Proposition 2.4.8, $(X, Y)_{\theta, \infty}$ is complete, then $(X, Y)_{\theta}$ is also complete.

The spaces $(X, Y)_{\theta, p}$ and $(X, Y)_{\theta}$ enjoy an important interpolation property, stated in the next proposition. It implies that they are in fact interpolation spaces.

Proposition 2.4.10. Let $X_{1}, X_{2}, Y_{1}, Y_{2}$ be Banach spaces, such that $Y_{i}$ is continuously embedded in $X_{i}$, for $i=1,2$. If $T \in \mathcal{L}\left(X_{1}, X_{2}\right) \cap \mathcal{L}\left(Y_{1}, Y_{2}\right)$, then $T \in \mathcal{L}\left(\left(X_{1}, Y_{1}\right)_{\theta, p}\right.$, $\left.\left(X_{2}, Y_{2}\right)_{\theta, p}\right) \cap \mathcal{L}\left(\left(X_{1}, Y_{1}\right)_{\theta},\left(X_{2}, Y_{2}\right)_{\theta}\right)$ for every $\theta \in(0,1)$ and $p \in[1, \infty]$, and for $(\theta, p)=$ $(1, \infty)$. Moreover,

$$
\|T\|_{\mathcal{L}\left(\left(X_{1}, Y_{1}\right)_{\theta, p},\left(X_{2}, Y_{2}\right)_{\theta, p}\right)} \leq\left(\|T\|_{\mathcal{L}\left(X_{1}, X_{2}\right)}\right)^{1-\theta}\left(\|T\|_{\mathcal{L}\left(Y_{1}, Y_{2}\right)}\right)^{\theta}
$$


Proof If $T=0$ the statement is trivial, so that we can assume that $T \neq 0$. Let $x \in\left(X_{1}, Y_{1}\right)_{\theta, p}$, then for every $a \in X_{1}, b \in Y_{1}$ such that $x=a+b$ and for every $t>0$ we have

$$
\|T a\|_{X_{2}}+t\|T b\|_{Y_{2}} \leq\|T\|_{\mathcal{L}\left(X_{1}, X_{2}\right)}\left(\|a\|_{X_{1}}+t \frac{\|T\|_{\mathcal{L}\left(Y_{1}, Y_{2}\right)}}{\|T\|_{\mathcal{L}\left(X_{1}, X_{2}\right)}}\|b\|_{Y_{1}}\right),
$$

so that

$$
K\left(t, T x, X_{2}, Y_{2}\right) \leq\|T\|_{\mathcal{L}\left(X_{1}, X_{2}\right)} K\left(t \frac{\|T\|_{\mathcal{L}\left(Y_{1}, Y_{2}\right)}}{\|T\|_{\mathcal{L}\left(X_{1}, X_{2}\right)}}, x, X_{1}, Y_{1}\right) .
$$

Setting $s=t \frac{\|T\|_{\mathcal{L}\left(Y_{1}, Y_{2}\right)}}{\|T\|_{\mathcal{L}\left(X_{1}, X_{2}\right)}}$ we get $T x \in\left(X_{2}, Y_{2}\right)_{\theta, p}$, and

$$
\|T x\|_{\left(X_{2}, Y_{2}\right)_{\theta, p}} \leq\|T\|_{\mathcal{L}\left(X_{1}, X_{2}\right)}\left(\frac{\|T\|_{\mathcal{L}\left(Y_{1}, Y_{2}\right)}}{\|T\|_{\mathcal{L}\left(X_{1}, X_{2}\right)}}\right)^{\theta}\|x\|_{\left(X_{1}, Y_{1}\right)_{\theta, p}},
$$

and (2.4.13) follows. From (2.4.14) it follows also that

$$
\lim _{t \rightarrow 0} t^{-\theta} K\left(t, x, X_{1}, Y_{1}\right)=0 \Rightarrow \lim _{t \rightarrow 0} t^{-\theta} K\left(t, T x, X_{2}, Y_{2}\right)=0,
$$

that is, $T$ maps $\left(X_{1}, Y_{1}\right)_{\theta}$ into $\left(X_{2}, Y_{2}\right)_{\theta}$.

Corollary 2.4.11. For $0<\theta<1,1 \leq p \leq \infty$ and for $(\theta, p)=(1, \infty)$ there is $c(\theta, p)$ such that

$$
\|y\|_{(X, Y)_{\theta, p}} \leq c(\theta, p)\|y\|_{X}^{1-\theta}\|y\|_{Y}^{\theta}, \quad \forall y \in Y .
$$

Proof Set $\mathbb{K}=\mathbb{R}$ or $\mathbb{K}=\mathbb{C}$, according to the fact that $X$ is a real or a complex Banach space. Let $y \in Y$, and define $T: \mathbb{K} \rightarrow X$, by $T(\lambda)=\lambda y$ for each $\lambda \in \mathbb{K}$. Then $\|T\|_{\mathcal{L}(\mathbb{K}, X)}=\|y\|_{X},\|T\|_{\mathcal{L}(\mathbb{K}, Y)}=\|y\|_{Y}$, and $\|T\|_{\mathcal{L}\left(\mathbb{K},(X, Y)_{\theta, p}\right)}=\|y\|_{(X, Y)_{\theta, p}}$. The statement follows now from Proposition 2.4.10, through the equality $(\mathbb{K}, \mathbb{K})_{\theta, p}=\mathbb{K}$.

The statement of Corollary 2.4.11 can be rephrased saying that every $(X, Y)_{\theta, p}$ belongs to $J_{\theta}(X, Y)$. In particular, $(X, Y)_{\theta, 1}$ belongs to $J_{\theta}(X, Y)$. We will see later (Proposition 2.4.17) that in fact a space $E$ belongs to the class $J_{\theta}(X, Y)$ if and only if $(X, Y)_{\theta, 1}$ is continuously embedded in $E$.

\subsubsection{The trace method}

We describe now another construction of the real interpolation spaces, which is one of the most common in the literature, see [23], and which will be useful for proving other properties.

Definition 2.4.12. For $0 \leq \theta<1$ and $1 \leq p \leq \infty$ set

$$
\begin{aligned}
V(p, \theta, Y, X)= & \left\{u: \mathbb{R}_{+} \rightarrow X ; t \mapsto u_{\theta}(t)=t^{\theta-1 / p} u(t) \in L_{p}(0,+\infty ; Y),\right. \\
& \left.t \mapsto v_{\theta}(t)=t^{\theta-1 / p} u^{\prime}(t) \in L_{p}(0,,+\infty ; X)\right\}, \\
& \|u\|_{V(p, \theta, Y, X)}=\left\|u_{\theta}\right\|_{L_{p}(0,+\infty ; Y)}+\left\|v_{\theta}\right\|_{L_{p}(0,+\infty ; X)} .
\end{aligned}
$$


Moreover, for $p=+\infty$ we define a subspace of $V(\infty, \theta, Y, X)$, by

$$
V_{0}(\infty, \theta, Y, X)=\left\{u \in V(\infty, \theta, Y, X) ; \lim _{t \rightarrow 0}\left\|t^{\theta} u(t)\right\|_{Y}=\lim _{t \rightarrow 0}\left\|t^{\theta} u^{\prime}(t)\right\|_{X}=0\right\} .
$$

It is not difficult to see that $V(p, \theta, Y, X)$ is a Banach space endowed with the norm $\|\cdot\|_{V(p, \theta, Y, X)}$, and that $V_{0}(\infty, \theta, Y, X)$ is a closed subspace of $V(\infty, \theta, Y, X)$. Moreover, if $\theta<1$, any function belonging to $V(p, \theta, Y, X)$ has a $X$-valued continuous extension at $t=0$. Indeed, for $0<s<t$, from the equality $u(t)-u(s)=\int_{s}^{t} u^{\prime}(\sigma) d \sigma$ it follows, for $1<p<\infty$,

$$
\begin{aligned}
\|u(t)-u(s)\|_{X} & \leq\left(\int_{s}^{t}\left\|\sigma^{\theta-1 / p} u^{\prime}(\sigma)\right\|_{X}^{p} d \sigma\right)^{1 / p}\left(\int_{s}^{t} \sigma^{-(\theta-1 / p) q} d \sigma\right)^{1 / q} \\
& \leq\|u\|_{V(p, \theta, Y, X)}[q(1-\theta)]^{-1 / q}\left(t^{q(1-\theta)}-s^{q(1-\theta)}\right)^{1 / q}
\end{aligned}
$$

with $q=p /(p-1)$. Arguing similarly, one sees that if $p=1$ or $p=\infty$, then $u$ is Lipschitz continuous (respectively, $(1-\theta)$-Hölder continuous) near $t=0$.

We shall use the Hardy-Young inequalities, which hold for every positive measurable function $\varphi:(0, a) \rightarrow \mathbb{R}, 0<a \leq \infty$, and every $\alpha>0, p \geq 1$, (see [19], p.245-246),

$$
\left\{\begin{array}{l}
\text { (i) } \quad \int_{0}^{a} t^{-\alpha p}\left(\int_{0}^{t} \varphi(s) \frac{d s}{s}\right)^{p} \frac{d t}{t} \leq \frac{1}{\alpha^{p}} \int_{0}^{a} s^{-\alpha p} \varphi(s)^{p} \frac{d s}{s} \\
\text { (ii) } \quad \int_{0}^{a} t^{\alpha p}\left(\int_{0}^{t} \varphi(s) \frac{d s}{s}\right)^{p} \frac{d t}{t} \leq \frac{1}{\alpha^{p}} \int_{0}^{a} s^{\alpha p} \varphi(s)^{p} \frac{d s}{s} .
\end{array}\right.
$$

Corollary 2.4.13. Let $u$ be a function such that $t \mapsto u_{\theta}(t)=t^{\theta-1 / p} u(t)$ belongs to $L_{p}(0, a ; X)$, with $0<a \leq \infty, 0<\theta<1$ and $1 \leq p \leq \infty$. Then also the mean value

$$
v(t)=\frac{1}{t} \int_{0}^{t} u(s) d s, \quad t>0
$$

has the same property, and setting $v_{\theta}=t^{\theta-1 / p} v(t)$ we have

$$
\left\|v_{\theta}\right\|_{L_{p}(0, a ; X)} \leq \frac{1}{1-\theta}\left\|u_{\theta}\right\|_{L_{p}(0, a ; X)}
$$

Proof For $p<\infty$, according to (2.4.18)(i), we have

$$
\begin{aligned}
\int_{0}^{a}\left\|v_{\theta}(t)\right\|_{X}^{p} d t & =\int_{0}^{a} t^{-(1-\theta) p}\left\|\int_{0}^{t} u(s) d s\right\|_{X}^{p} \frac{d t}{t} \\
& \leq \int_{0}^{a} t^{-(1-\theta) p}\left(\int_{0}^{t} s\|u(s)\|_{X} \frac{d s}{s}\right)^{p} \frac{d t}{t} \\
& \leq \frac{1}{(1-\theta)^{p}} \int_{0}^{a} s^{-(1-\theta) p}\left(s\|u(s)\|_{X}\right)^{p} \frac{d s}{s} \\
& \leq \frac{1}{(1-\theta)^{p}}\left\|u_{\theta}\right\|_{L_{p}(0, a ; X)}^{p},
\end{aligned}
$$


i.e. $v_{\theta}(t) \in L_{p}(0, a ; X)$, and

$$
\left\|v_{\theta}\right\|_{L_{p}(0, a ; X)} \leq \frac{1}{1-\theta}\left\|u_{\theta}\right\|_{L_{p}(0, a ; X)}
$$

For $p=\infty$, we have

$$
\begin{aligned}
\left\|v_{\theta}\right\|_{X} & \leq t^{\theta-1}\left\|\int_{0}^{t} u(s) d s\right\|_{X} \\
& \leq t^{\theta-1} \int_{0}^{t} s^{-\theta}\left\|s^{\theta} u(s)\right\|_{X} d s \\
& \leq \frac{1}{1-\theta}\left\|u_{\theta}\right\|_{L_{\infty}(0, a ; X)}
\end{aligned}
$$

This means $\left\|v_{\theta}\right\|_{L_{\infty}(0, a ; X)} \leq \frac{1}{1-\theta}\left\|u_{\theta}\right\|_{L_{\infty}(0, a ; X)}$. Thus, they complete the proof.

With the aid of Corollary 2.4.13, we are able to characterize the real interpolation spaces as trace spaces.

Proposition 2.4.14. For $(\theta, p) \in(0,1) \times[1,+\infty] \cup\{(1, \infty)\},(X, Y)_{\theta, p}$ is the set of the traces at $t=0$ of the functions in $V(p, 1-\theta, Y, X)$, and the norm

$$
\|x\|_{\theta, p}^{T}=\inf \left\{\|u\|_{V(p, 1-\theta, Y, X)} ; x=u(0), u \in V(p, 1-\theta, Y, X)\right\}
$$

is an equivalent norm in $(X, Y)_{\theta, p}$. Moreover, for $0<\theta<1,(X, Y)_{\theta}$ is the set of the traces of $t=0$ of the functions in $V_{0}(\infty, 1-\theta, Y, X)$.

Proof Let $x \in(X, Y)_{\theta, p}$. For every $n \in \mathbb{N}$, let $a_{n} \in X, b_{n} \in Y$ be such that $a_{n}+b_{n}=x$, and

$$
\left\|a_{n}\right\|_{X}+\frac{1}{n}\left\|b_{n}\right\|_{Y} \leq 2 K(1 / n, x)
$$

For $t>0$ set

$$
u(t)=\sum_{n=1}^{\infty} b_{n+1} \chi_{\left(\frac{1}{n+1}, \frac{1}{n}\right]}(t)=\sum_{n=1}^{\infty}\left(x-a_{n+1}\right) \chi_{\left(\frac{1}{n+1}, \frac{1}{n}\right]}(t)
$$

where $\chi_{I}$ is the characteristic function of the interval $I$, and

$$
v(t)=\frac{1}{t} \int_{0}^{t} u(s) d s
$$

Thanks to Proposition 2.4.7, $(X, Y)_{\theta, p} \subset(X, Y)_{\theta, \infty}$, then $\lim _{t \rightarrow 0} K(t, x)=0$. In particular, $\lim _{n \rightarrow \infty} a_{n}=0$ i.e. $x=\lim _{n \rightarrow \infty} b_{n}$, so that $x=\lim _{t \rightarrow 0} u(t)=\lim _{t \rightarrow 0} v(t)$. Moreover,

$$
\begin{aligned}
\left\|t^{1-\theta} u(t)\right\|_{Y} & \leq t^{1-\theta} \sum_{n=1}^{\infty} \chi_{\left(\frac{1}{n+1}, \frac{1}{n}\right]}(t) 2(n+1) K(1 /(n+1), x) \\
& \leq 2 t^{-\theta} \sup _{n \in \mathbb{N}}\left\{\frac{n+1}{n}\right\} K(t, x) \\
& \leq 4 t^{-\theta} K(t, x),
\end{aligned}
$$


so that $t \mapsto t^{1-\theta-1 / p} u(t) \in L_{p}(0,+\infty ; Y)$. By Corollary 2.4.13, $t \mapsto t^{1-\theta-1 / p} v(t)$ belongs to $L_{p}(0,+\infty ; Y)$, and

$$
\left\|t^{1-\theta-1 / p} v\right\|_{L_{p}(0,+\infty ; Y)} \leq \theta^{-1}\left\|t^{1-\theta-1 / p} u\right\|_{L_{p}(0,+\infty ; Y)} \leq 4 \theta^{-1}\|x\|_{\theta, p} .
$$

On the other hand,

$$
v(t)=x-\frac{1}{t} \int_{0}^{t} \sum_{n=1}^{\infty} \chi_{\left(\frac{1}{n+1}, \frac{1}{n}\right]}(s) a_{n+1} d s,
$$

so that $v$ is differentiable almost everywhere with values in $X$, and

$$
v^{\prime}(t)=\frac{1}{t^{2}} \int_{0}^{t} g(s) d s-\frac{1}{t} g(t)
$$

where $g(t)=\sum_{n=1}^{\infty} \chi_{\left(\frac{1}{n+1}, \frac{1}{n}\right]}(t) a_{n+1}$ is such that

$$
\|g(t)\|_{X} \leq \sum_{n=1}^{\infty} \chi_{\left(\frac{1}{n+1}, \frac{1}{n}\right]}(s) 2 K(1 /(n+1), x) \leq 2 K(t, x) .
$$

It follows that

$$
\left\|t^{1-\theta} v^{\prime}(t)\right\|_{X} \leq t^{-\theta} \sup _{0<s<t}\|g(s)\|_{X}+\left\|t^{-\theta} g(t)\right\|_{X} \leq 4 t^{-\theta} K(t, x) .
$$

Then $t \mapsto t^{1-\theta-1 / p} v^{\prime}(t)$ belongs to $L_{p}(0,+\infty ; X)$, and

$$
\left\|t^{1-\theta-1 / p} v^{\prime}(t)\right\|_{L_{p}(0,+\infty ; X)} \leq 4\|x\|_{\theta, p} .
$$

Therefore, $x$ is the trace at $t=0$ of a function $v \in V(p, 1-\theta, Y, X)$, and

$$
\|x\|_{\theta, p}^{T} \leq 4(1+1 / \theta)\|x\|_{\theta, p} .
$$

If $x \in(X, Y)_{\theta}$, then, by (2.4.21), $\lim _{t \rightarrow 0} t^{1-\theta}\|u(t)\|_{Y}=0$, so that $\lim _{t \rightarrow 0} t^{1-\theta}\|v(t)\|_{Y}=0$. By (2.4.22), $\lim _{t \rightarrow 0} t^{-\theta}\|g(t)\|_{X}=0$, so that $\lim _{t \rightarrow 0} t^{1-\theta}\left\|v^{\prime}(t)\right\|_{X}=0$. Then $v \in V_{0}(\infty, 1-$ $\theta, Y, X)$.

Conversely, let $x$ be the trace of a function $u \in V(p, 1-\theta, Y, X)$. Then

$$
x=x-u(t)+u(t)=-\int_{0}^{t} u^{\prime}(s) d s+u(t), \quad \forall t>0,
$$

so that

$$
t^{-\theta} K(t, x) \leq t^{1-\theta}\left\|\frac{1}{t} \int_{0}^{t} u^{\prime}(s) d s\right\|_{X}+t^{1-\theta}\|u(t)\|_{Y} .
$$

Corollary 2.4.13 implies now that $t \mapsto t^{-\theta-1 / p} K(t, x)$ belongs to $L_{p}(0,+\infty)$, so that $x \in(X, Y)_{\theta, p}$, and

$$
\|x\|_{\theta, p} \leq \frac{1}{\theta}\|x\|_{\theta, p}^{T}
$$

If $x$ is the trace of a function $u \in V_{0}(\infty, 1-\theta, Y, X)$, then, by $(2.4 .23), \lim _{t \rightarrow 0} t^{-\theta} K(t, x)$ $=0$, so that $x \in(X, Y)_{\theta}$. 
Remark 2.4.15. By Proposition 2.4.14, if $x \in(X, Y)_{\theta, p}$ or $x \in(X, Y)_{\theta}$, then $x$ is the trace at $t=0$ of a function $u$ belonging to $L_{p}(a, b ; Y) \cap W^{1, p}(a, b ; X)$ for $0<a<b$. But it is possible to find a more regular function $v \in V(p, 1-\theta, Y, X)$ (or $v \in V_{0}(\infty, 1-$ $\theta, Y, X))$ such that $v(0)=x$. For any $u \in V(p, 1-\theta, Y, X)\left(\right.$ or $\left.u \in V_{0}(\infty, 1-\theta, Y, X)\right)$ such that $u(0)=x$, set

$$
v(t)=\frac{1}{t} \int_{0}^{t} u(s) d s, \quad t>0 .
$$

Then $v \in W^{1, p}(a, b ; Y) \cap W^{2, p}(a, b ; X)$ for $0<a<b$, and $v(0)=x$. By Corollary 2.4.13, $t \mapsto t^{\theta-1 / p} v(t)$ belongs to $L_{p}(0,+\infty ; Y)$; moreover

$$
v^{\prime}(t)=-\frac{1}{t^{2}} \int_{0}^{t}(u(s)-u(t)) d s=-\frac{1}{t^{2}} \int_{0}^{t} d s \int_{s}^{t} u^{\prime}(\sigma) d \sigma
$$

so that

$$
\left\|v^{\prime}(t)\right\|_{X} \leq \frac{1}{t} \sup _{0<s<t}\left\|\int_{s}^{t} u^{\prime}(\sigma) d \sigma\right\|_{X} \leq \frac{1}{t} \int_{0}^{t}\left\|u^{\prime}(\sigma)\right\|_{X} d \sigma,
$$

and again by Corollary 2.4.13, $t \mapsto t^{1-\theta-1 / p} v^{\prime}(t)$ belongs to $L_{p}(0,+\infty ; X)$, and

$$
\|v\|_{V(p, 1-\theta, Y, X)} \leq \frac{1}{\theta}\|u\|_{V(p, 1-\theta, Y, X)}
$$

Moreover, $v^{\prime}(t)=(u(t)-v(t)) / t$, so that $t \mapsto t^{2-\theta-1 / p} v^{\prime}(t)$ belongs to $L_{p}(0,+\infty ; Y)$, and

$$
\left\|t^{2-\theta-1 / p} v^{\prime}\right\|_{L_{p}(0,+\infty ; Y)} \leq(1+1 / \theta)\left\|t^{1-\theta-1 / p} u\right\|_{L_{p}(0,+\infty ; Y)} .
$$

If $u \in V_{0}(\infty, 1-\theta, Y, X)$, it is easy to see that $v \in V_{0}(\infty, 1-\theta, Y, X)$, and that $\lim _{t \rightarrow 0} t^{2-\theta}\left\|v^{\prime}(t)\right\|_{Y}=0$.

By means of the trace method it is easy to prove some important density properties.

Proposition 2.4.16. Let $0<\theta<1$. For $1 \leq p<\infty, Y$ is dense in $(X, Y)_{\theta, p}$. For $p=\infty,(X, Y)_{\theta}$ is the closure of $Y$ in $(X, Y)_{\theta, \infty}$.

In the previous subsection we have seen that every $(X, Y)_{\theta, p}$ belongs to $J_{\theta}(X, Y)$. In particular, $(X, Y)_{\theta, 1}$ belongs to $J_{\theta}(X, Y)$. Now we can characterize all the spaces in the class $J_{\theta}(X, Y)$.

Proposition 2.4.17. Let $0<\theta<1$, and let $E$ be a Banach space such that $Y \subset E \subset$ $X$. The following statements are equivalent:

(i) E belongs to the class $J_{\theta}$ between $X$ and $Y$,

(ii) $(X, Y)_{\theta, 1} \subset E$. 
Proof The implication $(i i) \Rightarrow(i)$ is a straightforward consequence of Corollary 2.4.11, with $p=1$. Let us show that $(i) \Rightarrow(i i)$. For every $x \in(X, Y)_{\theta, 1}$, let $u \in V(1,1-\theta, Y, X)$ be such that $u(t)=0$ for $t \geq 1 . u(0)=x$, and set

$$
v(t)=\frac{1}{t} \int_{0}^{t} u(s) d s
$$

Then $v(0)=x, v(+\infty)=0$, so that

$$
x=-\int_{0}^{+\infty} v^{\prime}(t) d t
$$

Let $c$ be such that $\|y\|_{E} \leq c\|y\|_{X}^{1-\theta}\|y\|_{Y}^{\theta}$ for every $y \in Y$. Then

$$
\left\|v^{\prime}(t)\right\|_{E} \leq c\left\|v^{\prime}(t)\right\|_{X}^{1-\theta}\left\|v^{\prime}(t)\right\|_{Y}^{\theta}=c\left\|t^{-\theta} v^{\prime}(t)\right\|_{X}^{1-\theta}\left\|t^{1-\theta} v^{\prime}(t)\right\|_{Y}^{\theta} .
$$

By Remark 2.4.15, $t \mapsto t^{1-\theta} v^{\prime}(t)$ belongs to $L_{1}(0,+\infty ; Y)$, and $t \mapsto t^{-\theta} v^{\prime}(t)$ belongs to $L_{1}(0,+\infty ; X)$. By the Hölder inequality, $v^{\prime}$ belongs to $L_{1}(0,+\infty ; E)$, and, by means of $(2.4 .24)$ and (2.4.25),

$$
\begin{aligned}
\|x\|_{E} & \leq\left\|v^{\prime}(t)\right\|_{L_{1}(0, \infty ; E)} \\
& \leq c\left(\left\|t^{-\theta} v^{\prime}(t)\right\|_{L_{1}(0, \infty ; X)}\right)^{1-\theta}\left(\left\|t^{1-\theta} v^{\prime}(t)\right\|_{L_{1}(0, \infty ; Y)}\right)^{\theta} \\
& \leq c\left(\frac{1}{\theta}\|u\|_{V(1,1-\theta, Y, X)}\right)^{1-\theta}\left(\left(1+\frac{1}{\theta}\right)\|u\|_{V(1,1-\theta, Y, X)}\right)^{\theta} \\
& \leq \text { const. }\|u\|_{V(1,1-\theta, Y, X)} .
\end{aligned}
$$

Since $u$ is arbitrary and Proposition 2.4.14, we have $\|x\|_{E} \leq$ const. $\|x\|_{\theta, 1}$.

\subsubsection{The Reiteration Theorem}

We need some preliminaries about certain classes of intermediate spaces between $X$ and $Y$. Now we define another class of intermediate spaces.

Definition 2.4.18. Let $E$ be a Banach space such that $Y \subset E \subset X$, and let $0 \leq \theta \leq 1$. $E$ is said to belong to the class $K_{\theta}$ between $X$ and $Y$ if there is $k>0$ such that

$$
K(t, x) \leq k t^{\theta}\|x\|_{E}, \quad \forall x \in E, t>0 .
$$

In other words, $E$ belongs to the class $K_{\theta}$ if and only if it is continuously embedded in $(X, Y)_{\theta, \infty}$. In this case, we write $E \in K_{\theta}(X, Y)$.

By Definition 2.4.18 and Proposition 2.4.17, a space $E$ belongs to $K_{\theta}(X, Y) \cap$ $J_{\theta}(X, Y)$ if and only if

$$
(X, Y)_{\theta, 1} \subset E \subset(X, Y)_{\theta, \infty}
$$

Now we are able to state the Reiteration Theorem. 
Theorem 2.4.19. Let $0 \leq \theta_{0} \leq \theta_{1} \leq 1$. Fix $\theta \in(0,1)$ and set $\omega=(1-\theta) \theta_{0}+\theta \theta_{1}$. The following statements hold true.

(i) If $E_{i}$ belong to the class $K_{\theta_{i}}(i=0,1)$ between $X$ and $Y$, then

$$
\left(E_{0}, E_{1}\right)_{\theta, p} \subset(X, Y)_{\omega, p}, \quad \forall p \in[1, \infty], \quad\left(E_{0}, E_{1}\right)_{\theta} \subset(X, Y)_{\omega} .
$$

(ii) If $E_{i}$ belong to the class $J_{\theta_{i}}(i=0,1)$ between $X$ and $Y$, then

$$
(X, Y)_{\omega, p} \subset\left(E_{0}, E_{1}\right)_{\theta, p}, \quad \forall p \in[1, \infty], \quad(X, Y)_{\omega} \subset\left(E_{0}, E_{1}\right)_{\theta} .
$$

Consequently, if $E_{i}$ belong to $K_{\theta_{i}}(X, Y) \cap J_{\theta_{i}}(X, Y)$, then

$$
\left(E_{0}, E_{1}\right)_{\theta, p}=(X, Y)_{\omega, p}, \quad \forall p \in[1, \infty], \quad\left(E_{0}, E_{1}\right)_{\theta}=(X, Y)_{\omega}
$$

with equivalence of the respective norms.

Remark 2.4.20. By Proposition 2.4.7, $(X, Y)_{\theta, p}$ and $(X, Y)_{\theta}$ belong to $K_{\theta}(X, Y) \cap$ $J_{\theta}(X, Y)$ for $0<\theta<1$ and $1 \leq p \leq \infty$. The Reiteration Theorem yields

$$
\begin{aligned}
& \left((X, Y)_{\theta_{0}, q_{0}},(X, Y)_{\theta_{1}, q_{1}}\right)_{\theta, p}=(X, Y)_{(1-\theta) \theta_{0}+\theta \theta_{1}, p} \\
& \left((X, Y)_{\theta_{0}},(X, Y)_{\theta_{1}, q}\right)_{\theta, p}=(X, Y)_{(1-\theta) \theta_{0}+\theta \theta_{1}, p} \\
& \left((X, Y)_{\theta_{0}, q},(X, Y)_{\theta_{1}}\right)_{\theta, p}=(X, Y)_{(1-\theta) \theta_{0}+\theta \theta_{1}, p}
\end{aligned}
$$

for $0<\theta_{0}, \theta_{1}<1,1 \leq p, q \leq \infty$. Moreover, since $X$ belongs to $K_{0}(X, Y) \cap J_{0}(X, Y)$, and $Y$ belongs to $K_{1}(X, Y) \cap J_{1}(X, Y)$ between $X$ and $Y$, then

$$
\left((X, Y)_{\theta_{0}, q}, Y\right)_{\theta, p}=(X, Y)_{(1-\theta) \theta_{0}+\theta, p}, \quad\left((X, Y)_{\theta_{0}}, Y\right)_{\theta}=(X, Y)_{(1-\theta) \theta_{0}+\theta}
$$

and

$$
\left(X,(X, Y)_{\theta_{1}, q}\right)_{\theta, p}=(X, Y)_{\theta_{1} \theta, p}, \quad\left(X,(X, Y)_{\theta_{1}}\right)_{\theta}=(X, Y)_{\theta_{1} \theta},
$$

for $0<\theta_{0}, \theta_{1}<1,1 \leq p, q \leq \infty$. 


\section{Chapter 3}

\section{Preliminary II: Maximal Hölder Regularity}

\subsection{Analytic Semigroup}

Let $X$ be a complex Banach space, with norm $\|\cdot\|$. This section deals with the solution of an initial value problem in $X$,

$$
u^{\prime}(t)=A u(t), t>0 ; u(0)=x,
$$

where $A: D(A) \subset X \rightarrow X$ is a linear operator, with not necessarily dense domain.

Definition 3.1.1. Let $A: D(A) \subset X \rightarrow X$ be a linear operator. The resolvent set $\rho(A)$ and the spectrum $\sigma(A)$ of $A$ are defined by

$$
\rho(A)=\left\{\lambda \in \mathbb{C} ; \exists(\lambda I-A)^{-1} \in \mathcal{L}(X)\right\}, \quad \sigma(A)=\mathbb{C} \backslash \rho(A) .
$$

The complex numbers $\lambda \in \sigma(A)$ such that $\lambda I-A$ is not one to one are called eigenvalue. The set $\sigma_{p}(A)$ consisting of all eigenvalues of $A$ is called point spectrum.

If $\lambda \in \rho(A)$, we set

$$
(\lambda I-A)^{-1}=R(\lambda, A) .
$$

The operator $R(\lambda, A)$ is called resolvent operator or simply resolvent.

We state below some properties of the spectrum and the resolvent set.

First, it is clear that if $A: D(A) \subset X \rightarrow X$ and $B: D(B) \subset X \rightarrow X$ are linear operators such that $R\left(\lambda_{0}, A\right)=R\left(\lambda_{0}, B\right)$ for some $\lambda_{0} \in \mathbb{C}$, then $D(A)=D(B)$ and $A=B$. Indeed, $D(A)=$ Range $\left(R\left(\lambda_{0}, A\right)\right)=$ Range $\left(R\left(\lambda_{0}, B\right)\right)=D(B)$, and for every $x \in D(A)=D(B)$ we have

$$
R\left(\lambda_{0}, A\right)\left(\lambda_{0} x-A x\right)=x=R\left(\lambda_{0}, B\right)\left(\lambda_{0} x-B x\right)=R\left(\lambda_{0}, A\right)\left(\lambda_{0} x-B x\right),
$$

so that $\lambda_{0} x-A x=\lambda_{0} x-B x$, which implies $A x=B x$.

Next formula is called resolvent identity, its verification is straightforward:

$$
R(\lambda, A)-R(\mu, A)=(\mu-\lambda) R(\lambda, A) R(\mu, A), \quad \forall \lambda, \mu \in \rho(A) .
$$


Proposition 3.1.2. If $\rho(A)$ is not void, then $A$ is closed.

Proof Let $\left(x_{i}\right)$ be a sequence in $D(A)$, and

$$
\begin{aligned}
& x_{i} \longrightarrow x \quad \text { in } X, \\
& A x_{i} \longrightarrow y \quad \text { in } X .
\end{aligned}
$$

Since $\rho(A) \neq \emptyset$, there exists some $\lambda \in \rho(A)$ such that $R(\lambda, A) \in \mathcal{L}(X)$. Then

$$
x_{i}=R(\lambda, A)\left(\lambda x_{i}-A x_{i}\right) \longrightarrow R(\lambda, A)(\lambda x-y) .
$$

By the uniqueness, we have

$$
x=R(\lambda, A)(\lambda x-y) .
$$

This implies $x \in$ Range $(R(\lambda, A))=D(A)$. Moreover, $R(\lambda, A)$ is injective, we get

$$
\lambda x-A x=\lambda x-y .
$$

That is, $A x=y$. Thus, $A$ is closed.

Proposition 3.1.3. Let $\lambda_{0} \in \rho(A)$. Then the ball

$$
\left\{\lambda \in \mathbb{C} ;\left|\lambda-\lambda_{0}\right|<\left\|R\left(\lambda_{0}, A\right)\right\|_{\mathcal{L}(X)}^{-1}\right\}
$$

is contained in $\rho(A)$, and

$$
\begin{aligned}
R(\lambda, A) & =\sum_{n=0}^{\infty}(-1)^{n}\left(\lambda-\lambda_{0}\right)^{n} R^{n+1}\left(\lambda_{0}, A\right) \\
& =R\left(\lambda_{0}, A\right)\left[I+\left(\lambda-\lambda_{0}\right) R\left(\lambda_{0}, A\right)\right]^{-1} .
\end{aligned}
$$

Therefore, the resolvent set $\rho(A)$ is open in $\mathbb{C}$ and $\lambda \mapsto R(\lambda, A)$ is analytic in $\rho(A)$.

Definition 3.1.4. $A$ is said to be sectorial if there are constants $\omega \in \mathbb{R}, \theta \in\left(\frac{\pi}{2}, \pi\right)$ and $M>0$ such that

$$
\left\{\begin{array}{l}
(i) \quad \rho(A) \supset S_{\theta, \omega}=\{\lambda \in \mathbb{C} ; \lambda \neq \omega,|\arg (\lambda-\omega)|<\theta\} \\
(\text { ii }) \quad\|R(\lambda, A)\|_{\mathcal{L}(X)} \leq \frac{M}{|\lambda-\omega|} \quad \forall \lambda \in S_{\theta, \omega} .
\end{array}\right.
$$

The domain $D(A)$, endowed with the graph norm

$$
\|x\|_{D(A)}=\|x\|+\|A x\|,
$$

is a Banach space. For every $t>0,(3.1 .3)$ allows us to define a linear bounded operator $e^{t A}$ in $X$, by means of the Dunford integral

$$
e^{t A}=\frac{1}{2 \pi i} \int_{\omega+\gamma_{r, \eta}} e^{t \lambda} R(\lambda, A) d \lambda, \quad t>0
$$


where $r>0, \eta \in\left(\frac{\pi}{2}, \theta\right)$, and $\gamma_{r, \eta}$ is the curve $\{\lambda \in \mathbb{C} ;|\arg \lambda|=\eta,|\lambda| \geq r\} \cup\{\lambda \in$ $\mathbb{C} ;|\arg \lambda| \leq \eta,|\lambda|=r\}$, oriented counterclockwise. We also set

$$
e^{0 A} x=x, \quad \forall x \in X .
$$

Since the function $\lambda \mapsto e^{t \lambda} R(\lambda, A)$ is holomorphic in $S_{\theta, \omega}$, the definition of $e^{t A}$ is independent of the choice of $r$ and $\eta$.

Definition 3.1.5. Let $A: D(A) \subset X \rightarrow X$ be a sectorial operator. The family $\left\{e^{t A} ; t \geq 0\right\}$ defined by (3.1.4)-(3.1.5) is said to be the analytic semigroup generated by $A$ in $X$.

We recall that a family of linear operators $\{T(t)\}_{t \geq 0} \subset \mathcal{L}(X)$ is said to be a semigroup if

$$
\left\{\begin{array}{l}
T(t) T(s)=T(t+s), \quad t, s \geq 0, \\
T(0)=I .
\end{array}\right.
$$

A semigroup $T(t)$ is said to be analytic if the function $t \mapsto T(t)$ is analytic in $(0,+\infty)$ with values in $\mathcal{L}(X)$. It is said to be strongly continuous if for each $x \in X$ the function $t \mapsto T(t) x$ is continuous on $[0,+\infty)$. We shall show that if $A$ is sectorial, then $\left\{e^{t A}\right\}_{t \geq 0}$ is analytic, so that it is strongly continuous if and only if

$$
\lim _{t \rightarrow 0} e^{t A} x=x, \quad \forall x \in X
$$

We shall see later in Proposition 3.1.9 that

$$
\lim _{t \rightarrow 0} e^{t A} x=x \Longleftrightarrow x \in \overline{D(A)} .
$$

Therefore, $\left\{e^{t A}\right\}_{t \geq 0}$ is strongly continuous if and only if the domain $D(A)$ is dense in $X$.

\subsubsection{Basic properties of $e^{t A}$}

In this subsection, the proofs are omitted(cf. [24]).

Proposition 3.1.6. (i) $e^{t A} x \in D\left(A^{k}\right)$ for each $t>0, x \in X, k \in \mathbb{N}$. If $x \in D\left(A^{k}\right)$, then

$$
A^{k} e^{t A} x=e^{t A} A^{k} x, \quad \forall t \geq 0 .
$$

(ii) $e^{t A} e^{s A}=e^{(t+s) A}, \forall t, s \geq 0$.

(iii) There are constants $M_{0}, M_{1}, M_{2}, \ldots$, such that

$$
\left\{\begin{array}{l}
(a) \quad\left\|e^{t A}\right\|_{\mathcal{L}(X)} \leq M_{0} e^{\omega t}, \quad t>0, \\
(b) \quad\left\|t^{k}(A-\omega I)^{k} e^{t A}\right\|_{\mathcal{L}(X)} \leq M_{k} e^{\omega t}, \quad t>0,
\end{array}\right.
$$


where $\omega$ is the constant of assumption (3.1.3). In particular, from (3.1.6)(b), it follows that for every $\varepsilon>0$ and $k \in \mathbb{N}$ there is $C_{k, \varepsilon}>0$ such that

$$
\left\|t^{k} A^{k} e^{t A}\right\|_{\mathcal{L}(X)} \leq C_{k, \varepsilon} e^{(\omega+\varepsilon) t}, t>0 .
$$

(iv) The function $t \mapsto e^{t A}$ belongs to $C^{\infty}((0,+\infty) ; \mathcal{L}(X))$, and

$$
\frac{d^{k}}{d t^{k}} e^{t A}=A^{k} e^{t A}, \quad t>0,
$$

moreover, it has an analytic extension in the sector

$$
S \in\left\{\lambda \in \mathbb{C} ; \lambda \neq 0,|\arg \lambda|<\theta-\frac{\pi}{2}\right\} .
$$

Proposition 3.1.7. For every integer $n>\omega$, set

$$
A_{n}: X \rightarrow X, \quad A_{n}=n A R(n, A) .
$$

Then $\rho(A) \subset \rho\left(A_{n}\right)$, and $R\left(\lambda, A_{n}\right) \rightarrow R(\lambda, A)$ in $\mathcal{L}(X)$ as $n \rightarrow \infty$, for every $\lambda \in \rho(A)$. Moreover, $e^{t A_{n}} \rightarrow e^{t A}$ in $\mathcal{L}(X)$ as $n \rightarrow \infty$, for every $t>0$.

The family $\left\{A_{n} ; n \in \mathbb{N}\right\}$ is said to be the Yosida approximation of $A$.

Corollary 3.1.8. Let $X$ be a real Banach space, and let $A: D(A) \subset X \rightarrow X$ be a linear operator such that the complexification

$$
\widetilde{A}: D(\widetilde{A})=D(A)+i D(A) \rightarrow \widetilde{X}=X+i X, \quad \widetilde{A}(x+i y)=A x+i A y,
$$

is a sectorial operator in $\widetilde{X}$. Then $e^{t \widetilde{A}}(X) \subset X$.

The following proposition deals with the behavior of $e^{t A} x$ near $t=0$.

Proposition 3.1.9. The following statements hold true.

(i) If $x \in \overline{D(A)}$, then $\lim _{t \rightarrow 0^{+}} e^{t A} x=x$. Conversely, if there exists $y=\lim _{t \rightarrow 0^{+}} e^{t A} x$, then $x \in \overline{D(A)}$, and $y=x$.

(ii) For every $x \in X$ and $t \geq 0$, the integral $\int_{0}^{t} e^{s A} x d s$ belongs to $D(A)$, and

$$
A \int_{0}^{t} e^{s A} x d s=e^{t A} x-x .
$$

If in addition the function $s \mapsto A e^{s A} x$ belongs to $L_{1}(0, t ; X)$, then

$$
e^{t A} x-x=\int_{0}^{t} A e^{s A} x d s .
$$


(iii) If $x \in D(A)$ and $A x \in \overline{D(A)}$, then $\lim _{t \rightarrow 0^{+}}\left(e^{t A} x-x\right) / t=A x$. Conversely, if there exists $z=\lim _{t \rightarrow 0^{+}}\left(e^{t A} x-x\right) / t$, then $x \in D(A)$ and $z=A x \in \overline{D(A)}$.

(iv) If $x \in D(A)$ and $A x \in \overline{D(A)}$, then $\lim _{t \rightarrow 0^{+}} A e^{t A} x=A x$. Conversely, if there exists $v=\lim _{t \rightarrow 0^{+}} A e^{t A} x$, then $x \in D(A)$ and $v=A x \in \overline{D(A)}$.

If $X_{0}$ is a subspace of $X$, the part of $A$ in $X_{0}$ is defined by

$$
\left\{\begin{array}{l}
D\left(A_{0}\right)=\left\{x \in D(A) \cap X_{0} ; A x \in X_{0}\right\}, \\
A_{0}: D\left(A_{0}\right) \rightarrow X_{0}, \quad A_{0} x=A x .
\end{array}\right.
$$

Remark 3.1.10. Let $X_{0}=\overline{D(A)}$, and let $A_{0}$ be the part of $A$ in $X_{0}$. Then $D\left(A_{0}\right)$ is dense in $X_{0}$. Moreover, $A_{0}$ is sectorial so that it generates the analytic semigroup $e^{t A_{0}}$ in $X_{0}$, and we have $e^{t A_{0}} x=e^{t A} x$ for $x \in X_{0}$. Due to Proposition 3.1.9(i), $e^{t A_{0}}$ is strongly continuous in $X_{0}$.

\subsubsection{Identification of the generator}

Now we consider the problem of identifying the generator of a given analytic semigroup.

Lemma 3.1.11. Let $A: D(A) \subset X \rightarrow X$ satisfy (3.1.3). Then for every $\lambda \in \mathbb{C}$ such that $\operatorname{Re} \lambda>\omega$ we have

$$
R(\lambda, A)=\int_{0}^{+\infty} e^{-\lambda t} e^{t A} d t .
$$

Proof Let $0<r<R e \lambda-\omega$ and $\eta \in(\pi / 2, \theta)$. Then

$$
\begin{aligned}
\int_{0}^{+\infty} e^{-\lambda t} e^{t A} d t & =\frac{1}{2 \pi i} \int_{\omega+\gamma_{r, \eta}} R(z, A) \int_{0}^{+\infty} e^{-\lambda t+z t} d t d z \\
& =\frac{1}{2 \pi i} \int_{\omega+\gamma_{r, \eta}} R(z, A)(z-\lambda)^{-1} d z=R(\lambda, A) .
\end{aligned}
$$

Corollary 3.1.12. For every $t \geq 0, e^{t A}$ is one to one.

Corollary 3.1.13. If $A: D(A) \subset X \rightarrow X$ and $B: D(B) \subset X \rightarrow X$ are sectorial operators such that $e^{t A}=e^{t B}$ for every $t>0$, then $D(A)=D(B)$ and $A=B$.

Proposition 3.1.14. Let $\{T(t) ; t>0\}$ be a family of linear bounded operators such that $t \mapsto T(t)$ is differentiable with values in $\mathcal{L}(X)$, and

(i) $T(t) T(s)=T(t+s)$, for every $t, s>0$;

(ii) there are $\omega \in \mathbb{R}, M_{0}, M_{1}>0$ such that $\|T(t)\|_{\mathcal{L}(X)} \leq M_{0} e^{\omega t},\left\|t T^{\prime}(t)\right\|_{\mathcal{L}(X)} \leq M_{1} e^{\omega t}$ for $t>0$; 
(iii) either (a) there is $t>0$ such that $T(t)$ is one to one, or (b) for every $x \in X$, $\lim _{t \rightarrow 0} T(t) x=x$.

Then $t \mapsto T(t)$ is analytic in $(0,+\infty)$ with values in $\mathcal{L}(X)$, and there exists a unique sectorial operator $A: D(A) \subset X \rightarrow X$ such that $T(t)=e^{t A}$ for every $t \geq 0$.

Proof The proof can be found in [24] Proposition 2.1.9.

\subsubsection{A sufficient condition to be a sectorial operator}

Proposition 3.1.15. Let $A: D(A) \subset X \rightarrow X$ be a linear operator such that $\rho(A)$ contains a half plane $\{\lambda \in \mathbb{C} ; \operatorname{Re} \lambda \geq \omega\}$, and

$$
\|\lambda R(\lambda, A)\|_{\mathcal{L}(X)} \leq M, \quad \operatorname{Re} \lambda \geq \omega
$$

with $\omega \in \mathbb{R}, M>0$. Then $A$ is sectorial.

Proof By Proposition 3.1.3, for every $r>0$ the resolvent set of $A$ contains the open ball centered at $\omega+i r$ with radius $|\omega+i r| / M$. The union of such balls and $\rho(A)$ contains the sector $S=\{\lambda \in \mathbb{C} ; \lambda \neq \omega,|\arg (\lambda-\omega)|<\pi-\arctan M\}$. Moreover, for $\lambda \in V=\{\lambda \in \mathbb{C} ; \operatorname{Re} \lambda<\omega,|\arg (\lambda-\omega)| \leq \pi-\arctan 2 M\}, \lambda=\omega+i r-\theta r / M$ with $0<\theta \leq 1 / 2$, formula (3.1.2) gives

$$
\|R(\lambda, A)\|_{\mathcal{L}(X)} \leq \sum_{n=0}^{\infty}|\lambda-(\omega+i r)|^{n} \frac{M^{n+1}}{\left(\omega^{2}+r^{2}\right)^{(n+1) / 2}} \leq \frac{2 M}{r} .
$$

On the other hand, for $\lambda=\omega+i r-\theta r / M$ it holds

$$
r \geq\left(\frac{1}{4 M^{2}}+1\right)^{-1 / 2}|\lambda-\omega|
$$

so that $\|R(\lambda, A)\|_{\mathcal{L}(X)} \leq 2 M\left(\frac{1}{4 M^{2}}+1\right)^{1 / 2}|\lambda-\omega|^{-1}$. The statement follows.

Definition 3.1.16. Let $D \stackrel{d}{\hookrightarrow} X$, we define the following class

$$
\begin{aligned}
\mathcal{H}(D, X):= & \{A: D \rightarrow X ; \exists \omega \in \mathbb{R}, \exists M>0 \text { such that } \\
& \omega-A \in \mathcal{L} i s(D, X), \quad\{\lambda \in \mathbb{C} ; \operatorname{Re} \lambda \geq \omega\} \subset \rho(A) \\
& \text { and } \left.|\lambda-\omega|\|R(\lambda, A)\|_{\mathcal{L}(X)} \leq M, \text { for } \operatorname{Re} \lambda \geq \omega\right\}
\end{aligned}
$$

Given $A \in \mathcal{H}(D, X)$, we know from Proposition 3.1.9 and Proposition 3.1.15 that $A$ generates a strongly continuous analytic semigroup on $X$. In fact the converse is true as well: If $A$ generates a strongly continuous semigroup then $A$ belongs to $\mathcal{H}(D, X)$ (see [17] Section 4.2).

To prove the next proposition, we need the following lemma. 
Lemma 3.1.17. Let $X$ be a connected metric space, let

$$
B \in C(X, \mathcal{L}(E, F)),
$$

and suppose that there exists $\beta>0$ such that

$$
\|B(x) e\|_{F} \geq \beta\|e\|_{E}, \quad x \in X, \quad e \in E .
$$

Then $B(X) \cap \mathcal{L}$ is $(E, F) \neq \emptyset$ implies $B(X) \subset \mathcal{L}$ is $(E, F)$.

Proof The proof can be found in [4] Proposition 1.1.1.

Proposition 3.1.18. $\mathcal{H}(D, X)$ is open in $\mathcal{L}(D, X)$.

Proof For any $A \in \mathcal{H}(D, X)$, there exist $\omega=\omega(A) \in \mathbb{R}$ and $M=M(A)>0$ such that $\omega-A \in \mathcal{L}$ is $(D, X)$,

$$
\{\lambda \in \mathbb{C} ; \operatorname{Re} \lambda \geq \omega\} \subset \rho(A) \text { and }|\lambda-\omega|\|R(\lambda, A)\|_{\mathcal{L}(X)} \leq M, \text { for } \operatorname{Re} \lambda \geq \omega .
$$

For $\operatorname{Re} \lambda \geq \omega$, we have

$$
x=R(\omega, A)[(\lambda-A) x+(\omega-\lambda) x] \text { for } x \in D .
$$

Then for $x \in D$, we get

$$
\begin{aligned}
\|x\|_{D} & \leq\|R(\omega, A)\|_{\mathcal{L}(X, D)}(\|(\lambda-A) x\|+|\lambda-\omega|\|x\|) \\
& \leq\|R(\omega, A)\|_{\mathcal{L}(X, D)}(1+M)\|(\lambda-A) x\| .
\end{aligned}
$$

Set $K=M+\|R(\omega, A)\|_{\mathcal{L}(X, D)}(1+M)$, we know that for $x \in D$ and $R e \lambda \geq \omega$,

$$
|\lambda-\omega|\|x\|+\|x\|_{D} \leq K\|(\lambda-A) x\| .
$$

For any $B \in \mathcal{L}(D, X)$ with $\|B\|_{\mathcal{L}(D, X)} \leq \frac{1}{2 K}$, we see that

$$
\begin{aligned}
\|(\lambda-(A+B)) x\| & \geq\|(\lambda-A) x\|-\|B x\| \\
& \geq \frac{1}{K}\left(|\lambda-\omega|\|x\|+\|x\|_{D}\right)-\frac{1}{2 K}\|x\|_{D} \\
& =\frac{1}{K}|\lambda-\omega|\|x\|+\frac{1}{2 K}\|x\|_{D},
\end{aligned}
$$

for $x \in D$ and $\operatorname{Re} \lambda \geq \omega$. Since $D \stackrel{d}{\hookrightarrow} X$, (3.1.11) implies that $\{\lambda ; \operatorname{Re} \lambda \geq \omega\} \subset \rho(A+B)$ and

$$
|\lambda-\omega||| R(\lambda, A+B) \|_{\mathcal{L}(X)} \leq K \text { for } \operatorname{Re} \lambda \geq \omega .
$$

From (3.1.11), we deduce that

$$
\frac{1}{2 K}\|x\|_{D} \leq\|(\omega-(A+t B)) x\|, \quad x \in D, \quad 0 \leq t \leq 1 .
$$

Thanks to Lemma 3.1.17, we know that $\omega-(A+B) \in \mathcal{L} i s(D, X)$. Therefore $A+B \in$ $\mathcal{H}(D, X)$. This completes the proof. 


\subsubsection{Intermediate spaces}

Through the whole subsection we set

$$
C_{n}=\sup _{0<t \leq 1}\left\|t^{n} A^{n} e^{t A}\right\|_{\mathcal{L}(X)}, \quad \forall n \in \mathbb{N} .
$$

Then $C_{n}<+\infty$ for every $n$, thanks to estimates (3.1.7).

Proposition 3.1.19. For $0<k<n, D\left(A^{k}\right)$ belongs to the class $J_{k / n} \cap K_{k / n}$ between $X$ and $D\left(A^{n}\right)$.

Proof Since $D\left(A^{k}\right)=D\left((A-(\omega+1) I)^{k}\right)$ for every $k$, we may assume without loss of generality that $\omega<0$. Then the graph norm on $D\left(A^{k}\right)$ is equivalent to the norm $x \mapsto\left\|A^{k} x\right\|$, which will be used here.

Let us prove that $D\left(A^{k}\right) \in J_{k / n}\left(X, D\left(A^{n}\right)\right)$. First we consider the case $k=1, n=2$. We claim that there is $C>0$ such that

$$
\|A x\| \leq C\|x\|^{1 / 2}\left\|A^{2} x\right\|^{1 / 2}, \quad x \in D\left(A^{2}\right) .
$$

Let $x \in D\left(A^{2}\right)$. Since $\omega<0$, then $0 \in \rho\left(A^{2}\right)$, so that if $A^{2} x=0$ then $x=0$, and in this case (3.1.13) holds. Moreover, for every $t>0$ we have

$$
A e^{t A} x-A x=A^{2} \int_{0}^{t} e^{s A} x d s=\int_{0}^{t} e^{s A} A^{2} x d s
$$

so that, according to (3.1.6), we get

$$
\|A x\| \leq\left\|A e^{t A} x\right\|+\int_{0}^{t}\left\|e^{s A} A^{2} x\right\| d s \leq \frac{M_{1}}{t}\|x\|+M_{0} t\left\|A^{2} x\right\|, \quad t>0 .
$$

If $A^{2} x \neq 0$, taking the minimum of the right hand side for $t>0$ we get (3.1.13) with constant $C=2 \sqrt{M_{0} M_{1}}$. (3.1.13) means that $D(A) \in J_{1 / 2}\left(X, D\left(A^{2}\right)\right)$. Arguing by recurrence as in the proof of Proposition 2.4.2(i) one can see that $D\left(A^{k}\right) \in J_{k / n}\left(X, D\left(A^{n}\right)\right)$ for $0<k<n$.

To prove that $D\left(A^{k}\right) \in K_{k / n}\left(X, D\left(A^{n}\right)\right)$ we show first that $D(A) \in K_{1 / n}\left(X, D\left(A^{n}\right)\right)$. If $x \in D(A)$ split $x=e^{t^{1 / n} A} x+\left(x-e^{t^{1 / n} A} x\right)$, by means of Proposition 3.1.6, we have

$$
\left\|A^{n} e^{t^{1 / n} A} x\right\| \leq M_{n-1} t^{-1+1 / n}\|A x\|
$$

and

$$
\left\|x-e^{t^{1 / n}} A\right\|=\left\|\int_{0}^{t^{1 / n}} A e^{s A} x d s\right\| \leq M_{0} t^{1 / n}\|A x\|
$$

It follows that

$$
\begin{aligned}
K\left(t, x ; X, D\left(A^{n}\right)\right) & \leq\left\|x-e^{t^{1 / n} A} x\right\|+t\left\|e^{t^{1 / n} A} x\right\|_{D\left(A^{n}\right)} \\
& \leq M_{0} t^{1 / n}\|A x\|+C_{e q, n} M_{n-1} t^{1 / n}\|A x\| \\
& \leq C_{e q, 1}\left(M_{0}+C_{e q, n} M_{n-1}\right) t^{1 / n}\|x\|_{D(A)}
\end{aligned}
$$


for $t>0$, here, $C_{e q, k}$ is the coefficient concerned with equivalent norms between $\|\cdot\|_{D\left(A^{k}\right)}$ and $\left\|A^{k} \cdot\right\|$, i.e.

$$
C_{e q, k}^{-1}\left\|A^{k} x\right\| \leq\|x\|_{D\left(A^{k}\right)} \leq C_{e q, k}\left\|A^{k} x\right\|, \quad \forall k \in \mathbb{N}, x \in D\left(A^{k}\right) .
$$

Therefore, $D(A)$ is in the class $K_{1 / n}$ between $X$ and $D\left(A^{n}\right)$.

Let us argue by recurrence. Assume that for some $n \geq 3$ we have $D\left(A^{s}\right) \subset$ $\left(X, D\left(A^{n-1}\right)\right)_{s /(n-1), \infty}$ for $s=1, \ldots, n-2$. We have just proved that this is true for $n=3$. Set $Y=D(A)$. The part $A_{Y}$ of $A$ in $Y$ is sectorial, so that $D\left(A_{Y}^{s}\right) \subset$ $\left(Y, D\left(A_{Y}^{n-1}\right)\right)_{s /(n-1), \infty}$ for $s=1, \ldots, n-2$. On the other hand, $D\left(A_{Y}^{s}\right)=D\left(A^{s+1}\right)$, $D\left(A_{Y}^{n-1}\right)=D\left(A^{n}\right)$, so that $D\left(A^{s+1}\right) \subset\left(D(A), D\left(A^{n}\right)\right)_{s /(n-1), \infty}$. Since $D(A)$ belongs to $K_{1 / n}\left(X, D\left(A^{n}\right)\right)$, by part (i) of the Reiteration Theorem 2.4.19, we get

$$
\left(D(A), D\left(A^{n}\right)\right)_{s /(n-1), \infty} \subset\left(X, D\left(A^{n}\right)\right)_{(s+1) / n, \infty}
$$

for $1 \leq s \leq n-2$. Setting $s+1=k$ we get

$$
D\left(A^{k}\right) \subset\left(X, D\left(A^{n}\right)\right)_{k / n, \infty}, \quad 2 \leq k \leq n-1,
$$

and the statement follows.

In the following, we shall define a class of intermediate spaces between $X$ and $D(A)$ $(0<\alpha<1,1 \leq p \leq \infty$, and $(\alpha, p)=(1, \infty))$, by

$$
\left\{\begin{array}{r}
D_{A}(\alpha, p)=\left\{x \in X ; t \mapsto v(t)=\left\|t^{1-\alpha-1 / p} A e^{t A} x\right\| \in L_{p}(0,1)\right\}, \\
\|x\|_{D_{A}(\alpha, p)}=\|x\|+[x]_{D_{A}(\alpha, p)}=\|x\|+\|v\|_{L_{p}(0,1)} ; \\
D_{A}(\alpha)=\left\{x \in D_{A}(\alpha, \infty) ; \lim _{t \rightarrow 0} t^{1-\alpha} A e^{t A} x=0\right\} .
\end{array}\right.
$$

As easily seen, for every $x \in D_{A}(\alpha, p)$ and $T>0$, the function $s \mapsto\left\|A e^{s A} x\right\|$ belongs to $L_{1}(0, T)$, so that by Proposition 3.1.9(ii) we have

$$
e^{t A} x-x=\int_{0}^{t} A e^{s A} x d s \quad \forall t \geq 0, \quad x=\lim _{t \rightarrow 0} e^{t A} x .
$$

In particular, all the spaces $D_{A}(\alpha, p)$ and $D_{A}(\alpha)$ are contained in the closure of $D(A)$. Moreover we have

$$
D_{A}(\alpha, p)=D_{A_{0}}(\alpha, p), \quad D_{A}(\alpha)=D_{A_{0}}(\alpha),
$$

where $A_{0}$ is the part of $A$ in $\overline{D(A)}$. See Remark 3.1.10. In the following, we shall state several characterizations of the spaces $D_{A}(\alpha, p)$ and $D_{A}(\alpha)$.

First, the spaces $D_{A}(\alpha, p)$ and $D_{A}(\alpha)$ are real interpolation spaces between $X$ and $D(A)$.

Proposition 3.1.20. For $0<\alpha<1$ and $1 \leq p \leq \infty$, and for $(\alpha, p)=(1, \infty)$ we have

$$
D_{A}(\alpha, p)=(X, D(A))_{\alpha, p},
$$

with equivalence of the respective norms. Moreover, for $0<\alpha<1$,

$$
D_{A}(\alpha)=(X, D(A))_{\alpha} .
$$


Proof Let $\varphi:[0,+\infty) \rightarrow \mathbb{R}$ be a $C^{\infty}$ function such that

$$
\left\{\begin{array}{l}
0 \leq \varphi(t) \leq 1, \quad\left|\varphi^{\prime}(t)\right| \leq 2 \quad \forall t>0 \\
\varphi(t)=1 \text { for } 0 \leq t \leq 1 / 3, \quad \varphi(t)=0 \text { for } t \geq 1 .
\end{array}\right.
$$

Let $x \in D_{A}(\alpha, p)$. Then, choosing $u(t)=\varphi(t) e^{t A} x$, we get $x=u(0), u(t)=0$ for $t \geq 1$, and for $0<t \leq 1$

$$
\left\{\begin{array}{l}
\left\|t^{1-\alpha} u(t)\right\|_{D(A)} \leq\left\|t^{1-\alpha} A e^{t A} x\right\|+\left\|t^{1-\alpha} e^{t A} x\right\| \\
\left\|t^{1-\alpha} u^{\prime}(t)\right\| \leq\left\|t^{1-\alpha} A e^{t A} x\right\|+\left\|\varphi^{\prime}\right\|_{\infty}\left\|t^{1-\alpha} e^{t A} x\right\|
\end{array}\right.
$$

so that $u \in V(p, 1-\alpha, D(A), X)$, and

$$
\|u\|_{V(p, 1-\alpha, D(A), X)} \leq 2[x]_{D_{A}(\alpha, p)}+3\left\|t^{1-\alpha} e^{t A} x\right\|_{L_{p}(0,1 ; X)} .
$$

Due to Proposition 2.4.14, $x \in(X, D(A))_{\alpha, p}$, and

$$
\|x\|_{\alpha, p}^{T} \leq 2[x]_{D_{A}(\alpha, p)}+3 C_{0} c_{p}\|x\|
$$

where $c_{p}$ is a suitable constant. Therefore, $D_{A}(\alpha, p) \subset(X, D(A))_{\alpha, p}$. From the above considerations it is clear that if $x \in D_{A}(\alpha)$, then $u$ belongs to $V_{0}(\infty, 1-\alpha, D(A), X)$, so that $x \in(X, D(A))_{\alpha}$.

Conversely, let $x \in(X, D(A))_{\alpha, p}$. Then $x=u(0)$, with $u \in V(p, 1-\alpha, D(A), X)$. It follows that

$$
\begin{aligned}
\left\|t^{1-\alpha} A e^{t A} x\right\| & \leq\left\|t^{1-\alpha} A e^{t A} u(t)\right\|+\left\|t^{1-\alpha} A e^{t A} \int_{0}^{t} u^{\prime}(s) d s\right\| \\
& \leq C_{0}\left\|t^{1-\alpha} A u(t)\right\|+C_{1}\left\|t^{1-\alpha} \frac{1}{t} \int_{0}^{t} u^{\prime}(s) d s\right\| .
\end{aligned}
$$

Due to Corollary 2.4.13, $t \mapsto\left\|t^{1-\alpha-1 / p} A e^{t A} x\right\|$ belongs to $L_{p}(0,1)$, and

$$
\begin{aligned}
\left\|t^{1-\alpha-1 / p} A e^{t A} x\right\|_{L_{p}(0,1)} \leq & C_{0} \| \\
& +\frac{C_{1}^{1-\alpha-1 / p} A u(t) \|_{L_{p}(0,1)}}{\alpha}\left\|t^{1-\alpha-1 / p} u^{\prime}(t)\right\|_{L_{p}(0,1)} \\
\leq & \max \left\{C_{0}, \frac{C_{1}}{\alpha}\right\}\|u\|_{V(p, 1-\alpha, D(A), X)} .
\end{aligned}
$$

Since $u$ is arbitrary, we have

$$
\left\|t^{1-\alpha-1 / p} A e^{t A} x\right\|_{L_{p}(0,1)} \leq C\|x\|_{\alpha, p}^{T}
$$

Estimate (3.1.19) also holds for $p=\infty$, if we set $1 / \infty=0$. Therefore, $D_{A}(\alpha, p)$ is continuously embedded in $(X, D(A))_{\alpha, p}$ for $1 \leq p \leq \infty$.

If $x \in(X, D(A))_{\alpha}$, then $\left\|t^{1-\alpha} u(t)\right\|_{D(A)}$ and $\left\|t^{1-\alpha} u^{\prime}(t)\right\|$ go to 0 as $t \rightarrow 0$, and then it means that $\lim _{t \rightarrow 0} t^{1-\alpha} A e^{t A} x=0$, so that $x \in D_{A}(\alpha)$, and the statement is completely proved.

The above characterization yields immediately several properties of the spaces $D_{A}(\alpha, p)$ and $D_{A}(\alpha)$. 
Corollary 3.1.21. The following statements hold true.

(i) The spaces $D_{A}(\alpha, p)$ and $D_{A}(\alpha)$ do not depend explicitly on the operator $A$, but only on $D(A)$ and the graph norm of $A$. Precisely, if $B: D(B)=D(A) \rightarrow X$ is a sectorial operator such that

$$
c^{-1}\|A x\| \leq\|B x\| \leq c\|A x\| \quad \forall x \in D(A)
$$

for some $c \geq 1$, then we have (with equivalence of the respective norms)

$$
D_{B}(\alpha, p)=D_{A}(\alpha, p), \quad D_{B}(\alpha)=D_{A}(\alpha) .
$$

(ii) For $0<\alpha_{1}<\alpha_{2}<1,1 \leq p \leq \infty$, and for $\left(\alpha_{2}, p\right)=(1, \infty)$, we have

$$
D_{A}\left(\alpha_{2}, p\right) \subset D_{A}\left(\alpha_{1}, p\right) .
$$

For $0<\alpha<1,1 \leq p_{1}<p_{2}<\infty$

$$
D_{A}(1, \infty) \subset D_{A}\left(\alpha, p_{1}\right) \subset D_{A}\left(\alpha, p_{2}\right) \subset D_{A}(\alpha) \subset D_{A}(\alpha, \infty) \subset \overline{D(A)} .
$$

(iii) The spaces $D_{A}(\alpha, p)$ and $D_{A}(\alpha)$ belong to the class $J_{\alpha}$ between $X$ and $D(A)$. Moreover, if $E$ is a Banach space such that $D(A) \subset E \subset X$, then $E$ belongs to the class $J_{\alpha}$ between $X$ and $D(A)$ if and only if $D_{A}(\alpha, 1) \subset E$.

(iv) $D(A)$ is dense in $D_{A}(\alpha, p)$ for $p<\infty . D_{A}(\alpha)$ is the closure of $D(A)$ in $D_{A}(\alpha, \infty)$.

Proof Statement (i) is an obvious consequence of Proposition 3.1.20. Statements (ii), (iii) and (iv) follow from Proposition 3.1.20 through Proposition 2.4.7, Corollary 2.4.11 with Proposition 2.4.17, and Proposition 2.4.16, respectively.

The next proposition gives a characterization of the spaces $D_{A}(\alpha, p)$ and $D_{A}(\alpha)$ in terms of the behavior of the function $u(t)=e^{t A} x$ near $t=0$.

Proposition 3.1.22. It holds

$$
D_{A}(\alpha, p)=\left\{x \in X ; t \mapsto w(t)=t^{-\alpha-1 / p}\left\|e^{t A} x-x\right\| \in L_{p}(0,1)\right\},
$$

and, setting $[[x]]_{D_{A}(\alpha, p)}=\|w\|_{L_{p}(0,1)}$, the norm

$$
x \mapsto\|x\|+[[x]]_{D_{A}(\alpha, p)}
$$

is equivalent to the norm of $D_{A}(\alpha, p)$. Moreover,

$$
D_{A}(\alpha)=\left\{x \in X ; \lim _{t \rightarrow 0} t^{-\alpha}\left(e^{t A} x-x\right)=0\right\} .
$$


Remark 3.1.23. We remark that, in the case $p=\infty$, Proposition 3.1.22 states that $x \in$ $D_{A}(\alpha, \infty)$ if and only if the function $t \mapsto e^{t A} x$ belongs to $B U C^{\alpha}([0,1] ; X)$, if $0<\alpha<1$, or to $\operatorname{Lip}([0,1] ; X)$, if $\alpha=1$. This is clearly equivalent to $t \mapsto e^{t A} x \in \operatorname{BUC}^{\alpha}([0, T] ; X)$ (respectively, $\operatorname{Lip}([0, T] ; X))$ for all $T>0$.

The following proposition gives a characterization of $D_{A}(\alpha, p)$ and $D_{A}(\alpha)$ in terms of the behavior of $A R(\lambda, A) x$ as $\lambda \rightarrow+\infty$.

Proposition 3.1.24. Let $a>\max \{1, \omega\}$. Then

$$
D_{A}(\alpha, p)=\left\{x \in X ; t \mapsto z(t)=\left\|t^{\alpha-1 / p} A R(t, A) x\right\| \in L_{p}(a,+\infty)\right\},
$$

and, setting $[x]_{D_{A}(\alpha, p)}^{*}=\|z\|_{L_{p}(a,+\infty)}$, the norm

$$
x \mapsto\|x\|+[x]_{D_{A}(\alpha, p)}^{*}
$$

is equivalent to the norm $D_{A}(\alpha, p)$. Moreover,

$$
D_{A}(\alpha)=\left\{x \in X ; \lim _{t \rightarrow+\infty} t^{\alpha} A R(t, A) x=0\right\} .
$$

For $k \in \mathbb{N}, \alpha \in(0,1), p \in[1, \infty]$, set

$$
\begin{aligned}
& D_{A}(\alpha+k, p)=\left\{x \in D\left(A^{k}\right) ; A^{k} x \in D_{A}(\alpha, p)\right\} \text {, } \\
& \|x\|_{D_{A}(\alpha+k, p)}=\|x\|+\left\|A^{k} x\right\|_{D_{A}(\alpha, p)} ; \\
& D_{A}(\alpha+k)=\left\{x \in D\left(A^{k}\right) ; A^{k} x \in D_{A}(\alpha)\right\} \text {. }
\end{aligned}
$$

Thanks to Proposition 3.1.22, the function $t \mapsto e^{t A} x$ belongs to $B U C^{k+\alpha}([0,1]$; if and only if $x \in D_{A}(k+\alpha, \infty)$. Moreover, since $D(A)$ is dense in $D_{A}(\alpha, p)$ for $p<\infty$, then $D\left(A^{k+1}\right)$ is dense in $D_{A}(k+\alpha, p)$ for $p<\infty$; since $D_{A}(\alpha)$ is the closure of $D(A)$ in $D_{A}(\alpha, \infty)$, then $D_{A}(k+\alpha)$ is the closure of $D\left(A^{k+1}\right)$ in $D_{A}(k+\alpha, \infty)$.

A very important fact is that the parts of $A$ in $D_{A}(\alpha, p)$ and in $D_{A}(\alpha)$, defined by

$$
\begin{array}{ll}
A_{\alpha, p}: D_{A}(\alpha+1, p) \rightarrow D_{A}(\alpha, p), & A_{\alpha, p} x=A x \\
A_{\alpha}: D_{A}(\alpha+1) \rightarrow D_{A}(\alpha), & A_{\alpha} x=A x
\end{array}
$$

are sectorial operators in $D_{A}(\alpha, p)$ and in $D_{A}(\alpha)$, respectively.

Proposition 3.1.25. $\rho(A)$ is contained in $\rho\left(A_{\alpha, p}\right)$, and for every $\lambda \in \rho(A)$,

$$
\left\|R\left(\lambda, A_{\alpha, p}\right)\right\|_{\mathcal{L}\left(D_{A}(\alpha, p)\right)} \leq\|R(\lambda, A)\|_{\mathcal{L}(X)} .
$$

Consequently, $A_{\alpha, p}$ is a sectorial operator in $D_{A}(\alpha, p)$ and $A_{\alpha}$ is a sectorial operator in $D_{A}(\alpha)$.

We study now the behavior near $t=0$ of the function $t \mapsto e^{t A} x$, when $x \in D_{A}(\alpha, p)$ or $x \in D_{A}(\alpha)$. 
Proposition 3.1.26. For $0<\alpha<1$ and $1 \leq p<\infty$,

$$
\lim _{t \rightarrow 0}\left\|e^{t A} x-x\right\|_{D_{A}(\alpha, p)}=0, \quad \forall x \in D_{A}(\alpha, p) .
$$

For $0<\alpha<1$ and $x \in D_{A}(\alpha, \infty)$,

$$
\lim _{t \rightarrow 0}\left\|e^{t A} x-x\right\|_{D_{A}(\alpha, \infty)}=0 \Longleftrightarrow x \in D_{A}(\alpha) .
$$

Now we give some estimates for the function $t \mapsto A^{n} e^{t A}$ when $t \rightarrow 0$ and $t \rightarrow \infty$. For convenience, in the next proposition we set

$$
D_{A}(0, p)=X, \quad \forall p \in[1, \infty] .
$$

Proposition 3.1.27. Let $(\alpha, p),(\beta, p) \in(0,1) \times[1, \infty] \cup\{(1, \infty)\}$, and let $n \in \mathbb{N}$. Then there are $C=C(n, p ; \alpha, \beta), C^{\prime}=C^{\prime}(n, p ; \alpha, \beta)$ such that

$$
\left\{\begin{array}{l}
(i) \quad\left\|t^{n-\alpha+\beta} A^{n} e^{t A}\right\|_{\mathcal{L}\left(D_{A}(\alpha, p), D_{A}(\beta, p)\right)} \leq C, \quad 0<t \leq 1 \\
\text { (ii) }\left\|t^{n}(A-\omega I)^{n} e^{t A}\right\|_{\mathcal{L}\left(D_{A}(\alpha, p), D_{A}(\beta, p)\right)} \leq C^{\prime} e^{\omega t}, \quad t \geq 1 .
\end{array}\right.
$$

The statement holds also for $n=0$, provided $\alpha \leq \beta$.

Remark 3.1.28. In fact, we can also know that

$$
\sup _{0<t \leq 1}\left\|t^{n-\alpha+\beta} A^{n} e^{t A}\right\|_{\mathcal{L}\left(D_{A}(\alpha, \infty), D_{A}(\beta, p)\right)}<\infty, \quad \forall n \in \mathbb{N},
$$

for $0<\alpha, \beta<1,1 \leq p \leq \infty$. In particular, for $\alpha=\beta$,

$$
\sup _{0<t \leq 1}\left\|t^{n} A^{n} e^{t A}\right\|_{\mathcal{L}\left(D_{A}(\alpha, \infty), D_{A}(\alpha, p)\right)}<\infty, \quad \forall n \in \mathbb{N}, p \in[1, \infty] .
$$

This is not true in general for $n=0$.

Proposition 3.1.29. Let $0<\alpha<1$, and let I be a (possibly unbounded) interval. The following statements hold.

(i) If $u \in B U C^{\alpha}(I ; D(A)) \cap B U C^{1+\alpha}(I ; X)$, then $u^{\prime}(t) \in D_{A}(\alpha, \infty)$ for every $t \in I$, and

$$
\left\|u^{\prime}\right\|_{D_{A}(\alpha, \infty)} \leq C\left(\|u\|_{B U C^{\alpha}(I ; D(A))}+\|u\|_{B U C^{1+\alpha}(I ; X)}\right), \quad \forall t \in I .
$$

In addition, $B U C^{\alpha}(I ; D(A)) \cap B U C^{1+\alpha}(I ; X) \subset \operatorname{Lip}\left(I ; D_{A}(\alpha, \infty)\right)$.

(ii) $b u c^{\alpha}(I ; D(A)) \cap b u c^{1+\alpha}(I ; X) \subset B U C^{1}\left(I, D_{A}(\alpha)\right)$. 
Proof (i) First we shall prove that (3.1.23) holds. Let $u \in B U C^{\alpha}(I ; D(A)) \cap$ $B U C^{1+\alpha}(I ; X)$. For $t, t+h \in I$, split $u^{\prime}(t)$ as

$$
u^{\prime}(t)=\int_{0}^{1}\left(u^{\prime}(t)-u^{\prime}(t+\sigma h)\right) d \sigma+\frac{u(t+h)-u(t)}{h} .
$$

Then for every $\xi \in(0,1]$, it holds

$$
\begin{aligned}
& \left\|\xi^{1-\alpha} A e^{\xi A} u^{\prime}(t)\right\| \\
\leq & \xi^{1-\alpha}\left\|A e^{\xi A}\right\|_{\mathcal{L}(X)} \int_{0}^{1}\left\|u^{\prime}(t)-u^{\prime}(t+\sigma h)\right\| d \sigma \\
& +\xi^{1-\alpha}\left\|e^{\xi A}\right\|_{\mathcal{L}(X)}\left\|\frac{A u(t+h)-A u(t)}{h}\right\| \\
\leq & \frac{C_{1}}{\alpha+1} \xi^{-\alpha}\left[u^{\prime}\right]_{\alpha}^{X}|h|^{\alpha}+C_{0} \xi^{1-\alpha}[u]_{\alpha}^{D(A)}|h|^{\alpha_{1}} .
\end{aligned}
$$

If $I$ is unbounded, for every $\xi \in(0,1]$ and $t \in I$, there is $h \in \mathbb{R}$ such that $|h|=\xi$ and $t+h \in I$. Replacing in (3.1.24), we find

$$
\left\|\xi^{1-\alpha} A e^{\xi A} u^{\prime}(t)\right\| \leq \frac{C_{1}}{\alpha+1}\left[u^{\prime}\right]_{\alpha}^{X}+C_{0}[u]_{\alpha}^{D(A)},
$$

and estimate (3.1.23) holds. If $I=[a, b]$, and $\xi \leq(b-a) / 2$, for every $t \in(a, b]$, there is $h$ such that $|h|=\xi$ and $t+h \in[a, b]$. For such value of $\xi$, (3.1.25) holds. On the other hand, if $\xi>(b-a) / 2$, then

$$
\left\|\xi^{1-\alpha} A e^{\xi A} u^{\prime}(t)\right\| \leq \xi^{-\alpha} C_{1}\left\|u^{\prime}(t)\right\| \leq\left(\frac{2}{b-a}\right)^{\alpha} C_{1}\left\|u^{\prime}\right\|_{\infty}
$$

so that $\left[u^{\prime}\right]_{D_{A}(\alpha, \infty)}$ is bounded, and (3.1.23) is proved.

Let us show now that $u$ is Lipschitz continuous with values in $D_{A}(\alpha, \infty)$. For $0<$ $\xi \leq 1$ and $s<t \in I$, it holds

$$
\begin{aligned}
\left\|\xi^{1-\alpha} A e^{\xi A}(u(t)-u(s))\right\| & \leq \int_{s}^{t}\left\|\xi^{1-\alpha} A e^{\xi A} u^{\prime}(\sigma)\right\| d \sigma \\
& \leq(t-s) \sup _{\sigma \in I}\left[u^{\prime}(\sigma)\right]_{D_{A}(\alpha, \infty)}
\end{aligned}
$$

so that

$$
[u(t)-u(s)]_{D_{A}(\alpha, \infty)} \leq(t-s) \sup _{\sigma \in I}\left[u^{\prime}(\sigma)\right]_{D_{A}(\alpha, \infty)} .
$$

Let us prove that statement (ii) holds. If $u \in b u c^{\alpha}(I ; D(A)) \cap b u c^{1+\alpha}(I ; X)$, then there is a sequence of approximating functions $u_{n} \in B U C^{\infty}(I ; D(A))$ and converge to $u$ in $B U C^{\alpha}(I ; D(A)) \cap B U C^{1+\alpha}(I ; X)$. By statement (i), $\left\{u_{n}^{\prime}\right\}_{n \in \mathbb{N}}$ converges to $u^{\prime}$ in $L_{\infty}\left(I ; D_{A}(\alpha, \infty)\right)$. Since $B U C\left(I, D_{A}(\alpha)\right)$ is closed in $L_{\infty}\left(I ; D_{A}(\alpha, \infty)\right)$, then $u^{\prime} \in$ $B U C\left(I ; D_{A}(\alpha)\right)$. 


\subsection{Maximal Regularity}

In the section we shall study the solvability of the initial value problem

$$
u^{\prime}(t)=A u(t)+f(t), \quad t>0 ; \quad u(0)=u_{0},
$$

where $A$ is a linear sectorial operator in general Banach space $X$. The function $f$ is defined and continuous in a (possibly unbounded) interval $I$ such that inf $I=0$. We prove several properties of the solution in a bounded interval $[0, T]$. We also show that, under reasonable assumptions on the data, any solution of (3.2.1) in an arbitrary interval $[0, T]$ is given by the variation of constants formula

$$
u(t)=e^{t A} u_{0}+\int_{0}^{t} e^{(t-s) A} f(s) d s, \quad 0 \leq t \leq T .
$$

In this situation, the study of the solution of (3.2.1) is reduced to the study of the representation formula (3.2.2). Again we follow here [24], see also [9, 10].

Through the whole section, we fix $T>0$ and we set

$$
M_{k}=\sup _{0<t \leq T+1}\left\|t^{k} A^{k} e^{t A}\right\|_{\mathcal{L}(X)}, \quad k \in \mathbb{N} \cup\{0\},
$$

and, for $\alpha \in(0,1]$,

$$
\begin{array}{r}
M_{k, \alpha}=\sup _{0<t \leq T+1}\left\|t^{k-\alpha} A^{k} e^{t A}\right\|_{\mathcal{L}\left(D_{A}(\alpha, \infty), X\right), \quad k \in \mathbb{N},} \\
K_{k, \alpha}=\sup _{0<t \leq T+1}\left\|t^{k+\alpha} A^{k} e^{t A}\right\|_{\mathcal{L}\left(X, D_{A}(\alpha, 1)\right), \quad k \in \mathbb{N} \cup\{0\} .}
\end{array}
$$

Due to estimate (3.1.22), we have $M_{k}, M_{k, \alpha}, K_{k, \alpha}<\infty$ for every $k$.

Due to the singular behavior near $t=0$ of the derivatives of $t \mapsto e^{t A} x$, it will be sometimes convenient to work with weighted functional spaces. In particular, we shall consider spaces of functions defined in a bounded interval $(a, b]$, which are bounded or uniformly Hölder continuous in each interval $[a+\varepsilon, b]$ but that are not necessarily bounded (respectively, Hölder continuous) up to $t=a$. Let $\mu \in \mathbb{R}$, and set

$$
\begin{aligned}
& B_{\mu}((a, b] ; X)=\left\{f:(a, b] \rightarrow X ;\|f\|_{B_{\mu}((a, b] ; X)}\right. \\
&\left.=\sup _{a<t \leq b}(t-a)^{\mu}\|f(t)\|_{X}<\infty\right\} . \\
& C_{\mu}((a, b] ; X)= C((a, b] ; X) \cap B_{\mu}((a, b] ; X), \\
&\|f\|_{C_{\mu}((a, b] ; X)}=\|f\|_{B_{\mu}((a, b] ; X) .}
\end{aligned}
$$

For $0<\alpha<1, \beta>0$, set

$$
\begin{gathered}
C_{\beta}^{\alpha}((a, b] ; X)=\left\{f \in B_{\beta-\alpha}((a, b] ; X) \cap B U C^{\alpha}([a+\varepsilon, b] ; X), \forall \varepsilon \in(0, b-a) ;\right. \\
\left.[f]_{C_{\beta}^{\alpha}((a, b] ; X)}=\sup _{0<\varepsilon<b-a} \varepsilon^{\beta}[f]_{B U C^{\alpha}([a+\varepsilon, b] ; X)}<+\infty\right\} ; \\
\|f\|_{C_{\beta}^{\alpha}((a, b] ; X)}=\|f\|_{B_{\beta-\alpha}((a, b] ; X)}+[f]_{C_{\beta}^{\alpha}((a, b] ; X)} .
\end{gathered}
$$


In particular, for $\beta=\alpha$, the space $C_{\alpha}^{\alpha}((a, b] ; X)$ is the set of the bounded functions $f:(a, b] \rightarrow X$ such that

$$
\sup _{0<\varepsilon<b-a} \varepsilon^{\alpha}[f]_{B U C^{\alpha}([a+\varepsilon, b] ; X)}<+\infty
$$

It may be easily seen that it coincides with the set of the bounded functions $f$ : $(a, b] \rightarrow X$ such that $t \mapsto g(t)=(t-a)^{\alpha} f(t)$ belongs to $B U C^{\alpha}((a, b] ; X)$, and that the norm $[[f]]_{\alpha}=\|f\|_{\infty}+[g]_{B U C^{\alpha}((a, b] ; X)}$ is equivalent to the $C_{\alpha}^{\alpha}$ norm. More generally, one can show that $C_{\beta}^{\alpha}((a, b] ; X)$ is the space of the functions $f:(a, b] \rightarrow X$ such that $h(t)=(t-a)^{\beta-\alpha} f(t)$ is bounded and $g(t)=(t-a)^{\beta} f(t)$ is uniformly $\alpha$-Hölder continuous in $(a, b]$ with values in $X$, and that the norms $f \mapsto\|f\|_{C_{\beta}^{\alpha}((a, b] ; X)}$ and

$$
f \mapsto\|h\|_{B((a, b] ; X)}+[g]_{B U C^{\alpha}((a, b] ; X)}
$$

are equivalent. However, in what follows it is more convenient to use the norm defined in $(3.2 .8)$.

\subsubsection{Solutions of linear problems}

We consider several types of solutions of problem (3.2.1).

Definition 3.2.1. Let $T>0$, let $f:[0, T] \rightarrow X$ be a continuous function, and let $u_{0} \in X$. Then:

(i) A function $u \in C^{1}([0, T] ; X) \cap C([0, T] ; D(A))$ is said to be a strict solution of (3.2.1) in the interval $[0, T]$ if $u^{\prime}(t)=A u(t)+f(t)$ for each $t \in[0, T]$, and $u(0)=u_{0}$.

(ii) A function $u \in C([0, T] ; X)$ is said to be a strong solution of (3.2.1) in the interval $[0, T]$ if there is a sequence $\left\{u_{n}\right\}_{n \in \mathbb{N}} \subset C^{1}([0, T] ; X) \cap C([0, T] ; D(A))$ such that

$$
u_{n} \rightarrow u, \quad u_{n}^{\prime}-A u_{n} \rightarrow f \text { in } C([0, T] ; X) \text { as } n \rightarrow+\infty
$$

Let now $f:(0, T] \rightarrow X$ be continuous. Then

(iii) A function $u \in C^{1}((0, T] ; X) \cap C((0, T] ; D(A)) \cap C([0, T] ; X)$ is said to be a classical solution of (3.2.1) in the interval $[0, T]$ if $u^{\prime}(t)=A u(t)+f(t)$ for each $t \in(0, T]$, and $u(0)=u_{0}$.

If $f$ is defined in $[0,+\infty)$, a function $u:[0,+\infty) \rightarrow X$ is said to be a strict (respectively classical, strong) solution of $(3.2 .1)$ in $[0,+\infty)$ if for every $T>0$ the restriction $\left.u\right|_{[0, T]}$ is a strict (respectively classical, strong) solution of $(3.2 .1)$ in $[0, T]$. 
From Definition 3.2.1 it follows easily that if problem (3.2.1) has a strict solution then

$$
u_{0} \in D(A), \quad A u_{0}+f(0) \in \overline{D(A)},
$$

whereas if problem (3.2.1) has a classical or strong solution, then

$$
u_{0} \in \overline{D(A)} \text {. }
$$

Moreover, any strict solution is also strong and classical.

We are going to show that if $f \in L_{1}(0, T ; X)$ then any type of solution of $(3.2 .1)$ may be represented by the variation of constants formula (3.2.2). We begin with the classical solution.

Proposition 3.2.2. Let $f \in L_{1}(0, T ; X) \cap C((0, T] ; X)$, and let $u_{0} \in \overline{D(A)}$. If $u$ is a classical solution of (3.2.1), then

$$
u(t)=e^{t A} u_{0}+\int_{0}^{t} e^{(t-s) A} f(s) d s, \quad 0 \leq t \leq T .
$$

Proposition 3.2.2 implies that if $f \in L_{1}(0, T ; X) \cap C((0, T] ; X)$ then the classical solution of (3.2.1) is unique. In particular, if $f \in C([0, T] ; X)$ the strict solution of (3.2.1) is unique and it is given by (3.2.2). As a consequence, also the strong solution of (3.2.1) is unique, and it is given by (3.2.2).

In the applications we shall find situations in which $D(A)$ is not dense in $X$, and the initial datum $u_{0}$ does not belong to $\overline{D(A)}$. Then the initial condition $u(0)=u_{0}$ has to be understood in a weak sense, such as

$$
\lim _{t \rightarrow 0} R(\lambda, A) u(t)=R(\lambda, A) u_{0}
$$

for some $\lambda \in \rho(A)$. Also in this case the representation formula (3.2.2) holds, as the next corollary states.

Corollary 3.2.3. Let $f \in L_{1}(0, T ; X) \cap C((0, T] ; X)$ and $u_{0} \in X$. If $u \in C^{1}((0, T] ; X) \cap$ $C((0, T] ; D(A))$ satisfies $u^{\prime}(t)=A u(t)+f(t)$ for $t>0$, and $\lim _{t \rightarrow 0} R(\lambda, A) u(t)=R(\lambda, A) u_{0}$ for some $\lambda \in \rho(A)$, then $u$ is given by (3.2.2).

It is easy to see that (3.2.2) makes sense whenever $f \in L_{1}(0, T ; X)$ and $u_{0} \in X$. Therefore we give the following definition.

Definition 3.2.4. Let $f \in L_{1}(0, T ; X)$, and let $u_{0} \in X$. Then function $u$ defined in (3.2.2) is call the mild solution of (3.2.1).

Due to estimate (3.2.3), with $k=0$, the mild solution satisfies

$$
\|u(t)\| \leq M_{0}\left(\left\|u_{0}\right\|+\int_{0}^{t}\|f(s)\| d s\right), \quad 0 \leq t \leq T .
$$

It is easy to see that if $u_{0} \in \overline{D(A)}$ then $u$ belongs to $C([0, T] ; X)$.

The mild solution is also an integral solution, in the sense specified by the next proposition. 
Proposition 3.2.5. Let $f \in L_{1}(0, T ; X)$, and let $u_{0} \in X$. If $u$ is defined by (3.2.2), then for every $t \in[0, T]$ the integral $\int_{0}^{t} u(s) d s$ belongs to $D(A)$, and

$$
u(t)=u_{0}+A \int_{0}^{t} u(s) d s+\int_{0}^{t} f(s) d s, \quad 0 \leq t \leq T .
$$

The result of Proposition 3.2.5 is used in the next lemma, where we give sufficient conditions in order that a mild solution be classical or strict.

Lemma 3.2.6. Let $f \in L_{1}(0, T ; X) \cap C((0, T] ; X)$, let $u_{0} \in \overline{D(A)}$, and let $u$ be the mild solution of (3.2.1). The following conditions are equivalent.

(a) $u \in C((0, T] ; D(A))$,

(b) $u \in C^{1}((0, T] ; X)$,

(c) $u$ is a classical solution of (3.2.1).

If in addition $f \in C([0, T] ; X)$, then the following conditions are equivalent.

(a') $u \in C([0, T] ; D(A))$,

(b') $u \in C^{1}([0, T] ; X)$,

(c') $u$ is a strict solution of (3.2.1).

Now we discuss the relationship between mild and strong solutions. It is clear from the definition that if problem (3.2.1) has a strong solution, then $f \in C([0, T] ; X)$ and $u_{0} \in \overline{D(A)}$. Indeed, these conditions are also sufficient for the mild solution be strong, as the following proposition show.

Proposition 3.2.7. Let $f \in C([0, T] ; X), u_{0} \in \overline{D(A)}$. Then the mild solution of problem (3.2.1) is strong.

\subsubsection{Mild solutions}

This subsection is devoted to the properties of the mild solution of (3.2.1) in the case where $f$ belongs either to $L_{\infty}(0, T ; X)$ or to $C((0, T] ; X) \cap L_{1}(0, T ; X)$. Since the properties of the function $t \mapsto e^{t A} u_{0}$ were studied in last section, we focus our attention on the function

$$
v(t)=\left(e^{t A} * f\right)(t)=\int_{0}^{t} e^{(t-s) A} f(s) d s, \quad 0 \leq t \leq T .
$$

Proposition 3.2.8. Let $f \in L_{\infty}(0, T ; X)$. Then, for every $\alpha \in(0,1), v \in B U C^{\alpha}([0, T]$; $X) \cap C\left([0, T] ; D_{A}(\alpha, 1)\right)$. Precisely, it belongs to $B U C^{1-\alpha}\left([0, T] ; D_{A}(\alpha, 1)\right)$, and there is $C$ independent of $f$ such that

$$
\|v\|_{B U C^{1-\alpha}\left([0, T] ; D_{A}(\alpha, 1)\right)} \leq C\|f\|_{L_{\infty}(0, T ; X)} .
$$

It follows that $v$ belongs to $B U C^{1-\alpha}\left([0, T], X_{\alpha}\right)$ for every space $X_{\alpha} \in J_{\alpha}(X, D(A))$. 
Proof Since $s \mapsto\left\|e^{(t-s) A}\right\|_{\mathcal{L}\left(X, D_{A}(\alpha, 1)\right)}$ belongs to $L_{1}(0, t)$ for every $t \in(0, T]$, then $v(t)$ belongs to $D_{A}(\alpha, 1)$ for every $\alpha \in(0,1)$, and

$$
\begin{aligned}
\|v(t)\|_{D_{A}(\alpha, 1)} & \leq K_{0, \alpha}\|f\|_{L_{\infty}(0, T ; X)} \int_{0}^{t}(t-s)^{-\alpha} d s \\
& \leq K_{0, \alpha}(1-\alpha)^{-1} T^{1-\alpha}\|f\|_{L_{\infty}(0, T ; X)} .
\end{aligned}
$$

Moreover, for $0 \leq s<t \leq T$,

$$
\begin{aligned}
v(t)-v(s) & =\int_{0}^{s}\left(e^{(t-\sigma) A}-e^{(s-\sigma) A}\right) f(\sigma) d \sigma+\int_{s}^{t} e^{(t-\sigma) A} f(\sigma) d \sigma \\
& =\int_{0}^{s} d \sigma \int_{s-\sigma}^{t-\sigma} A e^{\tau A} d \tau+\int_{s}^{t} e^{(t-\sigma) A} f(\sigma) d \sigma
\end{aligned}
$$

which implies

$$
\begin{aligned}
\|v(t)-v(s)\|_{D_{A}(\alpha, 1)} \leq & K_{1, \alpha} \int_{0}^{s} d \sigma \int_{s-\sigma}^{t-\sigma} \tau^{-1-\alpha} d \tau\|f\|_{\infty} \\
& \quad+K_{0, \alpha} \int_{s}^{t}(t-\sigma)^{-\alpha} d \sigma\|f\|_{\infty} \\
\leq & \left(\frac{K_{1, \alpha}}{\alpha(1-\alpha)}+\frac{K_{0, \alpha}}{1-\alpha}\right)(t-s)^{1-\alpha}\|f\|_{\infty},
\end{aligned}
$$

so that $v$ is uniformly $(1-\alpha)$-Hölder continuous with values in $D_{A}(\alpha, 1)$. Estimate (3.2.14) follows now from (3.2.15) and (3.2.16).

Thanks to Proposition 2.4.17, we also get that $v$ is uniformly $(1-\alpha)$-Hölder continuous with values in $X_{\alpha}$ for $X_{\alpha} \in J_{\alpha}(X, D(A))$.

Corollary 3.2.9. Let $f \in L_{\infty}(0, T ; X), u_{0} \in X$, and let $u$ be the mild solution of (3.2.1). Then $u \in L_{\infty}(0, T ; X)$, and

$$
\|u\|_{L_{\infty}(0, T ; X)} \leq M_{0}\left(\left\|u_{0}\right\|+T\|f\|_{L_{\infty}(0, T ; X)}\right) .
$$

For every $\alpha \in(0,1)$, and $\varepsilon \in(0, T), u \in B U C^{1-\alpha}\left([\varepsilon, T] ; D_{A}(\alpha, 1)\right)$. Consequently, $u$ belongs to $B U C^{1-\alpha}\left([\varepsilon, T] ; X_{\alpha}\right)$ for every space $X_{\alpha} \in J_{\alpha}(X, D(A))$. There is $C$ independent of $f$ such that

$$
\begin{aligned}
& \|u\|_{B U C^{\alpha}([\varepsilon, T] ; X)}+\|u\|_{B\left([\varepsilon, T] ; D_{A}(\alpha, 1)\right)} \leq C\left(\varepsilon^{-\alpha}\left\|u_{0}\right\|+\|f\|_{L_{\infty}(0, T ; X)}\right), \\
& \|u\|_{B U C^{1-\alpha}\left([\varepsilon, T] ; D_{A}(\alpha, 1)\right)} \leq C\left(\varepsilon^{-1}\left\|u_{0}\right\|+\|f\|_{L_{\infty}(0, T ; X)}\right) .
\end{aligned}
$$

Moreover, $u \in C([0, T] ; X) \Longleftrightarrow u_{0} \in \overline{D(A)}$. For $0<\alpha<1, u \in B U C^{\alpha}([0, T], X) \Longleftrightarrow$ $u_{0} \in D_{A}(\alpha, \infty)$.

Proof This is a consequence of Proposition 3.2.8 and Remark 3.1.23.

In the applications to nonlinear problems we shall consider frequently functions $f$ which are bounded in every interval $[\varepsilon, T]$ with $\varepsilon \in(0, T)$, and blow up at $t=0$. Next proposition deals with functions belonging to the weighted spaces defined in (3.2.6), $(3.2 .8)$. 
Proposition 3.2.10. Let $f \in L_{1}(0, T ; X) \cap L_{\infty}(\varepsilon, T ; X)$ for every $\varepsilon \in(0, T)$. Then $v=e^{t A} * f$ belong to $C([0, T] ; X) \cap B U C^{1-\alpha}\left([\varepsilon, T] ; D_{A}(\alpha, 1)\right)$ for every $\alpha \in(0,1)$, $\varepsilon \in(0, T)$.

If in addition $t \mapsto t^{\theta} f(t)$ is bounded and measurable in $(0, T]$, for some $\theta \in(0,1)$ (so that $f \in B_{\theta}((0, T] ; X)$ ), then the following statements hold.

(i) $v \in B U C^{1-\theta}([0, T] ; X)$, and

$$
\|v\|_{B U C^{1-\theta}([0, T] ; X)} \leq C\|f\|_{B_{\theta}((0, T] ; X)} .
$$

(ii) For every $\alpha \in(0,1), v \in C_{\theta}^{1-\alpha}\left((0, T] ; D_{A}(\alpha, 1)\right)$, and

$$
\|v\|_{C_{\theta}^{1-\alpha}\left((0, T] ; D_{A}(\alpha, 1)\right)} \leq C\|f\|_{B_{\theta}((0, T] ; X)} .
$$

In particular, $v$ is bounded with values in $D_{A}(1-\theta, 1)$, and $t \mapsto t^{\theta+\alpha-1} v(t) \in$ $B\left([0, T] ; D_{A}(\alpha, 1)\right), t \mapsto t^{\theta} v(t) \in B U C^{1-\alpha}\left([0, T] ; D_{A}(\alpha, 1)\right)$ for every $\alpha \in(0,1)$. If in addition $\lim _{t \rightarrow 0}\left\|t^{\theta} f(t)\right\|=0$, then $v \in C\left([0, T] ; D_{A}(1-\theta, 1)\right)$.

(iii) If $\alpha+\theta<1$ then $v \in B U C^{1-\alpha-\theta}\left([0, T] ; D_{A}(\alpha, 1)\right)$, and

$$
\|v\|_{B U C^{1-\alpha-\theta}\left([0, T] ; D_{A}(\alpha, 1)\right)} \leq C\|f\|_{B_{\theta}((0, T] ; X)} .
$$

Proof Obviously, $v$ is continuous with values in $X$ up to $t=0$. For $0<\varepsilon \leq t \leq T$, we have

$$
v(t)=e^{(t-\varepsilon / 2) A} \int_{0}^{\varepsilon / 2} e^{(\varepsilon / 2-s) A} f(s) d s+\int_{\varepsilon / 2}^{t} e^{(t-s) A} f(s) d s=v_{1}(t)+v_{2}(t) .
$$

By Proposition 3.2.8, $v_{2}$ belongs to $B U C^{1-\alpha}\left([\varepsilon / 2, T] ; D_{A}(\alpha, 1)\right)$ for every $\alpha$. Moreover, $v_{1}$ belongs to $B U C^{\infty}\left([\varepsilon, T] ; D\left(A^{n}\right)\right)$ for every $n \in \mathbb{N}$, due to Proposition 3.1.6. Therefore, $v \in C([0, T] ; X) \cap B U C^{1-\alpha}\left([\varepsilon, T] ; D_{A}(\alpha, 1)\right)$.

Let now $t \mapsto t^{\theta} f(t)$ be measurable and bounded. Then for $0 \leq r<t \leq T$

$$
\begin{aligned}
\|v(t)-v(r)\| & \leq\left\|\int_{0}^{r}\left(e^{(t-s) A}-e^{(r-s) A}\right) f(s) d s\right\|+\left\|\int_{r}^{t} e^{(t-s) A} f(s) d s\right\| \\
& \leq\left(M_{1} \int_{0}^{r} \frac{d s}{s^{\theta}} \int_{r-s}^{t-s} \frac{d \sigma}{\sigma}+M_{0} \int_{r}^{t} \frac{d s}{s^{\theta}}\right)\|f\|_{B_{\theta}((0, T] ; X)} \\
& \leq\left(M_{1} \int_{0}^{r} \frac{d s}{(r-s)^{1-\theta} s^{\theta}} \int_{r-s}^{t-s} \frac{d \sigma}{\sigma^{\theta}}+M_{0} \int_{r}^{t} \frac{d s}{s^{\theta}}\right)\|f\|_{B_{\theta}((0, T] ; X)} \\
& \leq \frac{1}{1-\theta}\left(M_{1} \int_{0}^{1} \frac{d s}{(1-s)^{1-\theta} s^{\theta}}+M_{0}\right)(t-r)^{1-\theta}\|f\|_{B_{\theta}((0, T] ; X)}
\end{aligned}
$$

and statement (i) follows. 
Let us prove that statement (ii) holds. For $0<t \leq T$ we have

$$
\begin{aligned}
\|v(t)\|_{D_{A}(\alpha, 1)} & \leq K_{0, \alpha} \int_{0}^{t} \frac{d s}{(t-s)^{\alpha} s^{\theta}}\|f\|_{B_{\theta}((0, T] ; X)} \\
& =K_{0, \alpha} t^{1-\alpha-\theta} \int_{0}^{1} \frac{d s}{(1-s)^{\alpha} s^{\theta}}\|f\|_{B_{\theta}((0, T] ; X)}
\end{aligned}
$$

so that $t \mapsto t^{\theta+\alpha-1} v(t)$ is bounded in $(0, T]$ with values in $D_{A}(\alpha, 1)$. Moreover, if $N$ is such that $\left\|t A e^{t A}\right\|_{\mathcal{L}\left(D_{A}(\alpha, 1)\right)} \leq N$ for $0<t \leq T$, then for $0<\varepsilon \leq r<t \leq T$

$$
\begin{aligned}
& \left\|v_{1}(t)-v_{1}(r)\right\|_{D_{A}(\alpha, 1)}=\left\|\int_{r-\varepsilon / 2}^{t-\varepsilon / 2} A e^{\sigma A} d \sigma \int_{0}^{\varepsilon / 2} e^{(\varepsilon / 2-s) A} f(s) d s\right\|_{D_{A}(\alpha, 1)} \\
& \leq \frac{N K_{0, \alpha}}{(r-\varepsilon / 2)^{1-\alpha}} \int_{r-\varepsilon / 2}^{t-\varepsilon / 2} \frac{d \sigma}{\sigma^{\alpha}} \int_{0}^{1} \frac{d \tau}{(1-\tau)^{\alpha} \tau^{\theta}}\left(\frac{\varepsilon}{2}\right)^{1-\alpha-\theta}\|f\|_{B_{\theta}((0, T] ; X)} \\
& \leq \frac{N K_{0, \alpha}}{1-\alpha} \int_{0}^{1} \frac{d \sigma}{(1-\sigma)^{\alpha} \sigma^{\theta}}\left(\frac{\varepsilon}{2}\right)^{-\theta}(t-r)^{1-\alpha}\|f\|_{B_{\theta}((0, T] ; X)},
\end{aligned}
$$

and by estimate (3.2.16) there is $C>0$ independent of $\varepsilon$ such that

$$
\begin{aligned}
\left\|v_{2}(t)-v_{2}(r)\right\|_{D_{A}(\alpha, 1)} & \leq C(t-r)^{1-\alpha}\|f\|_{B([\varepsilon / 2, T] ; X)} \\
& \leq C(t-r)^{1-\alpha}\left(\frac{\varepsilon}{2}\right)^{-\theta}\|f\|_{B_{\theta}((0, T] ; X)}
\end{aligned}
$$

so that $v \in C_{\theta}^{1-\alpha}\left((0, T] ; D_{A}(\alpha, 1)\right)$. Choosing $\alpha=1-\theta$, we see that $v$ is bounded up to $t=0$ and continuous for $t>0$ with values in $D_{A}(1-\theta, 1)$. If in addition

$$
\lim _{t \rightarrow 0}\left\|t^{\theta} f(t)\right\|=0
$$

then $v$ is continuous up to $t=0$ with values in $D_{A}(1-\theta, 1)$. Indeed, for every $\varepsilon>0$ there is $\delta>0$ such that for $0<s \leq \delta$ we have $\left\|s^{\theta} f(s)\right\| \leq \varepsilon$, so that for $0<t \leq \delta$ estimate (3.2.19) can be replaced by

$$
\|v(t)\|_{D_{A}(1-\theta, 1)} \leq K_{0,1-\theta} \varepsilon \int_{0}^{1} \frac{d \sigma}{(1-\sigma)^{1-\theta} \sigma^{\theta}},
$$

which implies that $\lim _{t \rightarrow 0}\|v(t)\|_{D_{A}(1-\theta, 1)}=0$.

The proof of statement (iii) is similar to the one of statement (i).

In the next Corollary we combine the above results with the results of last section about the function $t \mapsto e^{t A} u_{0}$.

Corollary 3.2.11. Let $u_{0} \in X, f \in L_{1}(0, T ; X)$, and let $u$ be the mild solution of (3.2.1). Then $u$ belongs to $C((0, T] ; X)$ and

$$
\sup _{0 \leq t \leq T}\|u(t)\| \leq C\left(\left\|u_{0}\right\|+\|f\|_{L_{1}(0, T ; X)}\right) .
$$


Moreover, if $u_{0} \in D_{A}(\theta, \infty)$ and $t \mapsto t^{1-\theta} f(t)$ is measurable and bounded, for some $\theta \in(0,1)$, then $u \in B U C^{\theta}([0, T] ; X) \cap C_{1-\theta}^{1-\alpha}\left((0, T] ; D_{A}(\alpha, 1)\right)$ for every $\alpha \in(0,1)$ (so that in particular $\left.t \mapsto t^{1-\theta} u(t) \in B U C^{1-\alpha}\left([0, T] ; D_{A}(\alpha, 1)\right)\right)$, and

$$
\begin{aligned}
\|u\|_{B U C^{\theta}([0, T] ; X)} & \leq C\left(\left\|u_{0}\right\|_{D_{A}(\theta, \infty)}+\|f\|_{B_{1-\theta}((0, T] ; X)}\right), \\
\|u\|_{C_{1-\theta}^{1-\alpha}\left((0, T] ; D_{A}(\alpha, 1)\right)} & \leq C\left(\left\|u_{0}\right\|_{D_{A}(\theta, \infty)}+\|f\|_{B_{1-\theta}((0, T] ; X)}\right) .
\end{aligned}
$$

For $\alpha>\theta, t \mapsto t^{\alpha-\theta} u(t) \in B\left([0, T] ; D_{A}(\alpha, 1)\right)$, and

$$
\sup _{0 \leq t \leq T}\left\|t^{\alpha-\theta} u(t)\right\|_{D_{A}(\alpha, 1)} \leq C\left(\left\|u_{0}\right\|_{D_{A}(\theta, \infty)}+\|f\|_{B_{1-\theta}((0, T] ; X)}\right) .
$$

For $\alpha<\theta, u \in B U C^{\theta-\alpha}\left([0, T] ; D_{A}(\alpha, 1)\right)$, and

$$
\|u\|_{B U C^{\theta-\alpha}\left([0, T] ; D_{A}(\alpha, 1)\right)} \leq C\left(\left\|u_{0}\right\|_{D_{A}(\theta, \infty)}+\|f\|_{B_{1-\theta}((0, T] ; X)}\right) .
$$

Note that, if $u_{0}$ does not belong to the closure of $D(A)$, then $u$ is not continuous up to $t=0$. However, a mild continuity result at $t=0$ holds. See next proposition.

Proposition 3.2.12. Let $f \in L_{1}(0, T ; X), u_{0} \in X$, and let $u$ be the mild solution of (3.2.1). For every $\lambda \in \rho(A)$, it holds

$$
\lim _{t \rightarrow 0}\left\|R(\lambda, A)\left(u(t)-u_{0}\right)\right\|_{D_{A}(\theta, p)}=0,
$$

for each $\theta \in(0,1), p \geq 1$.

Proof The function $v=e^{t A} * f$ is continuous up to $t=0$ with values in $X$, so that $\lim _{t \rightarrow 0}\|R(\lambda, A) v(t)\|_{D_{A}(\theta, p)}=0$; moreover, since $R(\lambda, A) u_{0} \in D(A)$, then

$$
\lim _{t \rightarrow 0}\left\|R(\lambda, A)\left(e^{t A} u_{0}-u_{0}\right)\right\|_{D_{A}(\theta, p)}=\lim _{t \rightarrow 0}\left\|\left(e^{t A}-I\right) R(\lambda, A) u_{0}\right\|_{D_{A}(\theta, p)}=0 .
$$

\subsubsection{Time regularity}

The results of this subsection can be roughly grouped in two parts: the first part, concerning the case where $f$ is uniformly Hölder continuous up to $t=0$, and the second part, concerning the case where $f$ is uniformly Hölder continuous in each interval $[\varepsilon, T]$, with $0<\varepsilon<T$.

\section{The case where $f$ is uniformly Hölder continuous up to $t=0$}

Let $u$ be mild solution of (3.2.1), and set $u=u_{1}+u_{2}$, where

$$
\begin{cases}u_{1}(t)=\int_{0}^{t} e^{(t-s) A}(f(s)-f(t)) d s, & 0 \leq t \leq T \\ u_{2}(t)=e^{t A} u_{0}+\int_{0}^{t} e^{(t-s) A} f(t) d s, & 0 \leq t \leq T\end{cases}
$$


Theorem 3.2.13. Let $0<\alpha<1, f \in B U C^{\alpha}([0, T] ; X), u_{0} \in X$, and let $u$ be the mild solution of (3.2.1). Then $u$ belongs to $B U C^{\alpha}([\varepsilon, T] ; D(A)) \cap B U C^{1+\alpha}([\varepsilon, T] ; X)$ for every $\varepsilon \in(0, T)$, and

(i) if $u_{0} \in \overline{D(A)}$, then $u$ is a classical solution of (3.2.1);

(ii) if $u_{0} \in D(A)$ and $A u_{0}+f(0) \in \overline{D(A)}$, then $u$ is a strict solution of (3.2.1), and there is $C$ such that

$$
\|u\|_{C^{1}([0, T] ; X)}+\|u\|_{C([0, T] ; D(A))} \leq C\left(\|f\|_{B U C^{\alpha}([0, T] ; X)}+\left\|u_{0}\right\|_{D(A)}\right)
$$

(iii) if $u_{0} \in D(A)$ and $A u_{0}+f(0) \in D_{A}(\alpha, \infty)$, then both $u^{\prime}$ and $A u$ belong to $B U C^{\alpha}([0, T] ; X), u^{\prime}$ belongs to $B\left([0, T] ; D_{A}(\alpha, \infty)\right)$, and there is $C$ such that

$$
\begin{aligned}
& \|u\|_{B U C^{1+\alpha}([0, T] ; X)}+\|A u\|_{B U C^{\alpha}([0, T] ; X)}+\left\|u^{\prime}\right\|_{B\left([0, T] ; D_{A}(\alpha, \infty)\right)} \\
\leq & C\left(\|f\|_{B U C^{\alpha}([0, T] ; X)}+\left\|u_{0}\right\|_{D(A)}+\left\|A u_{0}+f(0)\right\|_{D_{A}(\alpha, \infty)}\right) .
\end{aligned}
$$

Proof Thanks to Lemma 3.2.6, to prove statement (i) and (ii), it is sufficient to show that $u$ belongs to $C((0, T] ; D(A))$ in the case where $u_{0} \in \overline{D(A)}$, and to $C([0, T] ; D(A))$ in the case where $u_{0} \in D(A)$ and $A u_{0}+f(0) \in \overline{D(A)}$.

Let $u_{1}$ and $u_{2}$ be defined by (3.2.20). Then $u_{1}(t) \in D(A)$ for $t \geq 0, u_{2}(t) \in D(A)$ for $t>0$, and

$$
\left\{\begin{array}{l}
\text { (i) } \quad A u_{1}(t)=\int_{0}^{t} A e^{(t-s) A}(f(s)-f(t)) d s, \quad 0 \leq t \leq T \\
(i i) \quad A u_{2}(t)=A e^{t A} u_{0}+\left(e^{t A}-1\right) f(t), \quad 0<t \leq T
\end{array}\right.
$$

If $u_{0} \in D(A)$, then (3.2.23)(ii) holds also for $t=0$.

Let us show that $A u_{1}$ is uniformly Hölder continuous in $[0, T]$. For $0 \leq s<t \leq T$,

$$
\begin{aligned}
A u_{1}(t)-A u_{1}(s)= & \int_{0}^{s} A\left(e^{(t-\sigma) A}-e^{(s-\sigma) A}\right)(f(\sigma)-f(s)) d \sigma \\
& +\left(e^{t A}-e^{(t-s) A}\right)(f(s)-f(t)) \\
& +\int_{s}^{t} A e^{(t-\sigma) A}(f(\sigma)-f(t)) d \sigma
\end{aligned}
$$

so that

$$
\begin{aligned}
& \left\|A u_{1}(t)-A u_{1}(s)\right\| \\
\leq & M_{2} \int_{0}^{s}(s-\sigma)^{\alpha} \int_{s-\sigma}^{t-\sigma} \tau^{-2} d \tau d \sigma[f]_{\alpha}+2 M_{0}(t-s)^{\alpha}[f]_{\alpha} \\
& +M_{1} \int_{s}^{t}(t-\sigma)^{\alpha-1} d \sigma[f]_{\alpha} \\
\leq & M_{2} \int_{0}^{s} d \sigma \int_{s-\sigma}^{t-\sigma} \tau^{\alpha-2} d \tau[f]_{\alpha}+\left(2 M_{0}+M_{1} \alpha^{-1}\right)(t-s)^{\alpha}[f]_{\alpha} \\
\leq & \left(\frac{M_{2}}{\alpha(1-\alpha)}+2 M_{0}+\frac{M_{1}}{\alpha}\right)(t-s)^{\alpha}[f]_{\alpha} .
\end{aligned}
$$


Therefore, $A u_{1}$ is uniformly $\alpha$-Hölder continuous in $[0, T]$. Moreover, $A u_{2}$ is obviously continuous in $(0, T]$ : hence, if $u_{0} \in \overline{D(A)}$, then $u \in C([0, T] ; X)$, and $A u \in C((0, T] ; X)$, so that, by Lemma 3.2.6, $u$ is a classical solution of (3.2.1), and statement (i) is proved.

If $u_{0} \in D(A)$ we have

$$
A u_{2}(t)=e^{t A}\left(A u_{0}+f(0)\right)+e^{t A}(f(t)-f(0))-f(t), \quad 0 \leq t \leq T,
$$

so that if $A u_{0}+f(0) \in \overline{D(A)}$, then $A u_{2}$ is continuous also at $t=0$, and statement (ii) follows.

In the case where $A u_{0}+f(0) \in D_{A}(\alpha, \infty)$, from (3.2.26) we get, for $0 \leq s<t \leq T$,

$$
\begin{aligned}
& \left\|A u_{2}(t)-A u_{2}(s)\right\| \\
\leq & \left\|\left(e^{t A}-e^{s A}\right)\left(A u_{0}+f(0)\right)\right\|+\left\|\left(e^{t A}-e^{s A}\right)(f(s)-f(0))\right\| \\
& +\left\|\left(e^{t A}-1\right)(f(t)-f(s))\right\| \\
\leq & \int_{s}^{t}\left\|A e^{\sigma A}\right\|_{\mathcal{L}\left(D_{A}(\alpha, \infty), X\right)} d \sigma\left\|A u_{0}+f(0)\right\|_{D_{A}(\alpha, \infty)} \\
& +s^{\alpha}\left\|A \int_{s}^{t} e^{\sigma A} d \sigma\right\|_{\mathcal{L}(X)}[f]_{\alpha}+\left(M_{0}+1\right)(t-s)^{\alpha}[f]_{\alpha} \\
\leq & \frac{M_{1, \alpha}}{\alpha}\left\|A u_{0}+f(0)\right\|_{D_{A}(\alpha, \infty)}(t-s)^{\alpha} \\
& +\left(\frac{M_{1}}{\alpha}+M_{0}+1\right)(t-s)^{\alpha}[f]_{\alpha},
\end{aligned}
$$

so that also $A u_{2}$ is uniformly $\alpha$-Hölder continuous, and the estimate

$$
\begin{aligned}
& \|u\|_{B U C^{1+\alpha}([0, T] ; X)}+\|A u\|_{B U C^{\alpha}([0, T] ; X)} \\
\leq & C\left(\|f\|_{B U C^{\alpha}([0, T] ; X)}+\left\|u_{0}\right\|_{D(A)}+\left\|A u_{0}+f(0)\right\|_{D_{A}(\alpha, \infty)}\right)
\end{aligned}
$$

follows easily.

Since $u^{\prime}$ and $A u$ are uniformly $\alpha$-Hölder continuous, from Proposition 3.1.29(i), it follows that $u^{\prime}$ is bounded with values in $D_{A}(\alpha, \infty)$. However, the embedding constant given by Proposition 3.1.29(i) depends on the length $T$ of the interval in such a way that it blows up as $T \rightarrow 0$. So we estimate $\left[u^{\prime}(t)\right]_{D_{A}(\alpha, \infty)}$ directly. For $0 \leq t \leq T$ we have, by (3.2.23),

$$
u^{\prime}(t)=\int_{0}^{t} A e^{(t-s) A}(f(s)-f(t)) d s+e^{t A}\left(A u_{0}+f(0)\right)+e^{t A}(f(t)-f(0)),
$$

so that for $0<\xi \leq 1$,

$$
\begin{aligned}
& \left\|\xi^{1-\alpha} A e^{\xi A} u^{\prime}(t)\right\| \\
\leq & \left\|\xi^{1-\alpha} \int_{0}^{t} A^{2} e^{(t+\xi-s) A}(f(s)-f(t)) d s\right\| \\
& +\left\|\xi^{1-\alpha} A e^{(t+\xi) A}\left(A u_{0}+f(0)\right)\right\|+\left\|\xi^{1-\alpha} A e^{(t+\xi) A}(f(t)-f(0))\right\| \\
\leq & M_{2} \xi^{1-\alpha} \int_{0}^{t}(t-s)^{\alpha}(t+\xi-s)^{-2} d s[f]_{\alpha} \\
& +M_{0}\left[A u_{0}+f(0)\right]_{D_{A}(\alpha, \infty)}+M_{1} \xi^{1-\alpha}(t+\xi)^{-1} t^{\alpha}[f]_{\alpha} \\
\leq & M_{2} \int_{0}^{+\infty} \sigma^{\alpha}(\sigma+1)^{-2} d \sigma[f]_{\alpha}+M_{0}\left[A u_{0}+f(0)\right]_{D_{A}(\alpha, \infty)}+M_{1}[f]_{\alpha} .
\end{aligned}
$$


Therefore, $\left[u^{\prime}(t)\right]_{D_{A}(\alpha, \infty)}$ is bounded in $[0, T]$, and the proof is complete.

Corollary 3.2.14. Let $0<\alpha<1$, and let $f \in b u c^{\alpha}([0, T] ; X), u_{0} \in D(A)$. Assume moreover that

$$
A u_{0}+f(0) \in D_{A}(\alpha) .
$$

Then the solution $u$ of problem (3.2.1) belongs to buc ${ }^{1+\alpha}([0, T] ; X) \cap b u c^{\alpha}([0, T] ; D(A))$, and $u^{\prime}$ belongs to $C\left([0, T] ; D_{A}(\alpha)\right)$.

Proof Let $\left\{f_{n}\right\}_{n \in \mathbb{N}} \subset B U C^{\infty}([0, T] ; X)$ be such that $f_{n} \rightarrow f$ in $B U C^{\alpha}([0, T] ; X)$. Let $\lambda \in \rho(A)$ be fixed. Since $D\left(A^{2}\right)$ is dense in $D_{A}(\alpha+1)$, and $u_{0}+(A-\lambda I)^{-1} f(0)=$ $(A-\lambda I)^{-1}\left(A u_{0}+f(0)-\lambda u_{0}\right) \in D_{A}(\alpha+1)$, thanks to (3.2.29), there is a sequence $\left\{y_{n}\right\}_{n \in \mathbb{N}} \subset D\left(A^{2}\right)$ such that $y_{n} \rightarrow u_{0}+(A-\lambda I)^{-1} f(0)$ in $D_{A}(\alpha+1, \infty)$. Set

$$
x_{n}=y_{n}-(A-\lambda I)^{-1} f_{n}(0), \quad n \in \mathbb{N} .
$$

Then $x_{n} \in D(A)$, and $A x_{n}+f_{n}(0)=A y_{n}-\lambda(A-\lambda I)^{-1} f_{n}(0) \in D(A)$. By means of Theorem 3.2.13, the solution $u_{n}$ of problem

$$
u_{n}^{\prime}(t)=A u_{n}(t)+f_{n}(t), \quad 0 \leq t \leq T ; \quad u_{n}(0)=x_{n}
$$

belongs to $B U C^{1+\alpha+\varepsilon}([0, T] ; X) \cap B U C^{\alpha+\varepsilon}([0, T] ; D(A))$, and $u_{n}^{\prime} \in B\left([0, T] ; D_{A}(\alpha+\right.$ $\varepsilon, \infty))$ for every $\varepsilon \in(0,1-\alpha)$, so that $u_{n} \in b u c^{1+\alpha}([0, T] ; X) \cap b u c^{\alpha}([0, T] ; D(A))$ and $u_{n}^{\prime} \in C\left([0, T] ; D_{A}(\alpha)\right)$ thanks to Proposition 3.1.29(ii). Moreover,

$$
\begin{gathered}
f_{n} \rightarrow f \text { in } B U C^{\alpha}([0, T] ; X), \quad x_{n} \rightarrow u_{0} \text { in } D(A), \\
A x_{n}+f_{n}(0) \rightarrow A u_{0}+f(0) \text { in } D_{A}(\alpha, \infty),
\end{gathered}
$$

thus, due again to Theorem 3.2.13, $u_{n} \rightarrow u$ in $B U C^{1+\alpha}([0, T] ; X) \cap B U C^{\alpha}([0, T] ; D(A))$ as $n \rightarrow \infty$, and $\left\{u_{n}^{\prime}\right\}_{n \in \mathbb{N}}$ is a Cauchy sequence in $C\left([0, T], D_{A}(\alpha)\right)$. The statement follows now easily.

Remark 3.2.15. From Proposition 3.1.29, it follows that if $f \in B U C^{\alpha}([0, T] ; X)$ then the condition $A u_{0}+f(0) \in D_{A}(\alpha, \infty)$ is necessary to get $u^{\prime}, A u \in B U C^{\alpha}([0, T] ; X)$, and if $f \in \operatorname{buc}^{\alpha}([0, T] ; X)$ then the condition $A u_{0}+f(0) \in D_{A}(\alpha)$ is necessary to get $u^{\prime}, A u \in b u c^{\alpha}([0, T] ; X)$.

\section{The case where $f$ is not uniformly Hölder continuous up to $t=0$}

In many applications to nonlinear or nonautonomous problems we have to deal with functions $f$ that are not necessarily uniformly Hölder continuous up to $t=0$.

Theorem 3.2.16. Let $0<\alpha<1, f \in L_{1}(0, T ; X) \cap B U C^{\alpha}([\varepsilon, T] ; X)$ for every $\varepsilon \in(0, T)$, and let $u_{0} \in X$. Then the mild solution $u$ of problem (3.2.1) belongs to $C((0, T] ; D(A)) \cap C^{1}((0, T] ; X) \cap B U C^{\alpha}([\varepsilon, T] ; D(A)) \cap B U C^{1+\alpha}([\varepsilon, T] ; X)$, for every $\varepsilon \in(0, T)$. If in addition $u_{0} \in \overline{D(A)}$, then $u$ is a classical solution. 
Proof Let us split $u$ as $u(t)=e^{t A} u_{0}+v(t)$, where $v(t)=e^{t A} * f$. The first term is obviously continuous in $(0, T]$ with values in $D(A)$, and it belongs to $C([0, T] ; X)$ if and only if $u_{0} \in \overline{D(A)}$. So, we consider only the function $v$.

We showed in Proposition 3.2.10 that $v$ is continuous in $[0, T]$ with values in $X$. Moreover, for $0<\varepsilon \leq t \leq T$,

$$
v(t)=e^{(t-\varepsilon / 2) A} v(\varepsilon / 2)+\int_{\varepsilon / 2}^{t} e^{(t-s) A} f(s) d s=v_{1}(t)+v_{2}(t),
$$

where $v_{1} \in B U C^{\infty}\left([\varepsilon, T] ; D\left(A^{n}\right)\right)$ for every $n \in \mathbb{N}, v_{2} \in B U C^{\alpha}([\varepsilon, T] ; D(A)) \cap B U C^{1+\alpha}$ $([\varepsilon, T] ; X)$ thanks to Theorem 3.2.13. Since $\varepsilon$ is arbitrary, $u \in C((0, T] ; D(A)) \cap$ $C^{1}((0, T] ; X)$.

If in addition $u_{0} \in \overline{D(A)}$, then $u \in C([0, T] ; X)$ and it is a classical solution thanks to Lemma 3.2.6. The statement follows.

If we have more precise information on the behavior of the Hölder seminorm of $f$ near $t=0$, we can study the behavior of $u$ near $t=0$. In the next theorem we assume that $f$ belongs to the weighted Hölder space $C_{\alpha}^{\alpha}((0, T] ; X)$, or to $C([0, T] ; X) \cap$ $C_{\alpha}^{\alpha}((0, T] ; X)$, with $0<\alpha<1$. We recall that $f \in C_{\alpha}^{\alpha}((0, T] ; X)$ means that $f$ is bounded and that $t \mapsto t^{\alpha} f(t)$ is $\alpha$-Hölder continuous in $(0, T]$. Weighted Hölder spaces naturally arise in the study of parabolic evolution equations: for instance, the function

$$
t \mapsto e^{t A} x, \quad 0 \leq t \leq T,
$$

belongs to $C_{\alpha}^{\alpha}((0, T] ; X)$ for all $x \in X$, and to $C([0, T], X) \cap C_{\alpha}^{\alpha}((0, T] ; X)$ for all $x \in \overline{D(A)}$.

Theorem 3.2.17. Let $0<\alpha<1, f \in C_{\alpha}^{\alpha}((0, T] ; X)$, and set $v=e^{t A} * f$. Then $v \in$ $C_{\alpha}^{\alpha}((0, T] ; D(A))$, it is differentiable in $(0, T]$ with values in $X$, and $v^{\prime} \in C_{\alpha}^{\alpha}((0, T] ; X) \cap$ $B_{\alpha}\left((0, T] ; D_{A}(\alpha, \infty)\right)$. There is $C>0$ such that

$$
\left\|v^{\prime}\right\|_{C_{\alpha}^{\alpha}(X)}+\|A v\|_{C_{\alpha}^{\alpha}(X)}+\left\|v^{\prime}\right\|_{B_{\alpha}\left(D_{A}(\alpha, \infty)\right)} \leq C\|f\|_{C_{\alpha}^{\alpha}(X)} .
$$

Proof By Theorem 3.2.16, $v$ belongs to $C((0, T] ; D(A)) \cap C^{1}((0, T] ; X)$. Let us show that it is bounded with values in $D(A)$. For $0 \leq t \leq T$ we have

$$
\|v(t)\| \leq M_{0} T\|f\|_{L_{\infty}(0, T ; X)} .
$$

Moreover, using the decomposition

$$
v(t)=\int_{0}^{t} e^{(t-s) A}(f(s)-f(t)) d s+\int_{0}^{t} e^{\sigma A} f(t) d \sigma,
$$

we get

$$
\begin{aligned}
\|A v(t)\| & \leq M_{1} \int_{0}^{t}(t-s)^{\alpha-1} s^{-\alpha} d s[f]_{C_{\alpha}^{\alpha}(X)}+\left\|\left(e^{t A}-I\right) f(t)\right\| \\
& \leq M_{1} \int_{0}^{1}(1-s)^{\alpha-1} s^{-\alpha} d s[f]_{C_{\alpha}^{\alpha}(X)}+\left(M_{0}+1\right)\|f\|_{\infty} .
\end{aligned}
$$


Thus,

$$
\|v(t)\|_{D(A)} \leq C_{1}\|f\|_{C_{\alpha}^{\alpha}((0, T] ; X)}, \quad 0<t \leq T .
$$

As a second step, we prove that $t^{\alpha} v^{\prime}(t)$ is bounded with values in $D_{A}(\alpha, \infty)$, which means that $v^{\prime} \in B_{\alpha}\left((0, T] ; D_{A}(\alpha, \infty)\right)$. Since

$$
v^{\prime}(t)=A v(t)+f(t)=\int_{0}^{t} A e^{(t-s) A}(f(s)-f(t)) d s+e^{t A} f(t),
$$

then for each $t \in(0, T]$ and $\xi \in(0,1]$ we have

$$
\begin{aligned}
& t^{\alpha} \xi^{1-\alpha}\left\|A e^{\xi A} v^{\prime}(t)\right\| \\
\leq & t^{\alpha}\left\|\xi^{1-\alpha} \int_{0}^{t / 2} A^{2} e^{(t+\xi-s) A}(f(s)-f(t)) d s\right\| \\
& +t^{\alpha} \xi^{1-\alpha}\left(\left\|\int_{t / 2}^{t} A^{2} e^{(t+\xi-s) A}(f(s)-f(t)) d s\right\|+\left\|A e^{(t+\xi) A} f(t)\right\|\right) \\
\leq & M_{2} t^{\alpha} \xi^{1-\alpha} \int_{0}^{t / 2}(t+\xi-s)^{-2} d s 2\|f\|_{\infty} \\
& +M_{2} t^{\alpha} \xi^{1-\alpha} \int_{t / 2}^{t} \frac{(t-s)^{\alpha}}{s^{\alpha}(t+\xi-s)^{2}} d s[f]_{C_{\alpha}^{\alpha}(X)}+M_{1} t^{\alpha} \xi^{1-\alpha}(t+\xi)^{-1}\|f(t)\| \\
\leq & 2^{\alpha+1} M_{2}\|f\|_{\infty}+2^{\alpha} M_{2} \int_{0}^{t / 2} \sigma^{\alpha}(\sigma+1)^{-2} d \sigma[f]_{C_{\alpha}^{\alpha}(X)}+M_{1}\|f\|_{\infty} .
\end{aligned}
$$

Hence, $t^{\alpha} v^{\prime}(t)$ is bounded in $(0, T]$ with values in $D_{A}(\alpha, \infty)$, which implies that $v^{\prime}$ belongs to $B_{\alpha}\left((0, T] ; D_{A}(\alpha, \infty)\right)$. Further, there is $C_{2}$ such that

$$
\left\|v^{\prime}\right\|_{B_{\alpha}\left((0, T] ; D_{A}(\alpha, \infty)\right)} \leq C_{2}\|f\|_{C_{\alpha}^{\alpha}((0, T] ; X)}
$$

Let us prove that $v \in C_{\alpha}^{\alpha}((0, T] ; D(A)$. For every $\varepsilon \in(0, T)$ it holds

$$
v(t)=e^{(t-\varepsilon) A} v(\varepsilon)+\int_{\varepsilon}^{t} e^{(t-s) A} f(s) d s, \quad \varepsilon \leq t \leq T .
$$

Since $f \in B U C^{\alpha}([\varepsilon, T] ; X)$ and $v(\varepsilon) \in D(A), A v(\varepsilon)+f(\varepsilon)=v^{\prime}(\varepsilon) \in D_{A}(\alpha, \infty)$, then from Theorem 3.2.13(iii), applied in the interval $[\varepsilon, T]$ instead of $[0, T]$, it follows that $v \in B U C^{\alpha}([\varepsilon, T] ; D(A)) \cap B U C^{1+\alpha}([\varepsilon, T] ; X)$, and that $v^{\prime} \in B\left([\varepsilon, T] ; D_{A}(\alpha, \infty)\right)$. From estimate $(3.2 .22)$ we get

$$
\begin{aligned}
& \|v\|_{B U C^{\alpha}([\varepsilon, T] ; D(A))}+\left\|v^{\prime}\right\|_{B U C^{\alpha}([\varepsilon, T] ; X)}+\left\|v^{\prime}\right\|_{B\left([\varepsilon, T] ; D_{A}(\alpha, \infty)\right)} \\
\leq & C\|v(\varepsilon)\|_{D(A)}+\left\|v^{\prime}(\varepsilon)\right\|_{D_{A}(\alpha, \infty)}+\|f\|_{B U C^{\alpha}([\varepsilon, T] ; X)} \\
\leq & C\left(C_{1}\|f\|_{C_{\alpha}^{\alpha}((0, T] ; X)}+\frac{C_{2}}{\varepsilon^{\alpha}}\|f\|_{C_{\alpha}^{\alpha}((0, T] ; X)}+\frac{1}{\varepsilon^{\alpha}}\|f\|_{C_{\alpha}^{\alpha}((0, T] ; X)}\right)
\end{aligned}
$$

so that $v \in C_{\alpha}^{\alpha}((0, T] ; D(A))$, and estimate (3.2.30) follows.

Corollary 3.2.18. Let $0<\alpha<1, f \in C_{\alpha}^{\alpha}((0, T] ; X), u_{0} \in X$, and let $u$ be the mild solution of (3.2.1). The following statements hold. 
(i) If $u_{0} \in \overline{D(A)}$, then $u$ is a classical solution of (3.2.1);

(ii) if $u_{0} \in D_{A}(1, \infty)$, then $u^{\prime}$ and $A$ u belong to $C_{\alpha}^{\alpha}((0, T] ; X), u^{\prime}$ belongs to $B_{\alpha}((0, T]$; $\left.D_{A}(\alpha, \infty)\right)$, and there is $C>0$ such that

$$
\begin{gathered}
\left\|u^{\prime}\right\|_{C_{\alpha}^{\alpha}((0, T] ; X)}+\|A u\|_{C_{\alpha}^{\alpha}((0, T] ; X)}+\left\|u^{\prime}\right\|_{B_{\alpha}\left((0, T] ; D_{A}(\alpha, \infty)\right)} \\
\leq C\left(\|f\|_{C_{\alpha}^{\alpha}((0, T] ; X)}+\left\|u_{0}\right\|_{D_{A}(1, \infty)}\right)
\end{gathered}
$$

(iii) if $f \in C([0, T] ; X), u_{0} \in D(A)$, and $A u_{0}+f(0) \in \overline{D(A)}$, then $u^{\prime}, A u \in C([0, T] ; X)$ and $u$ is a strict solution of problem (3.2.1).

Proof Statement (i) follows obviously from Proposition 3.1.9(i) and Theorem 3.2.17.

To prove statement (ii) we have to check that for every $x \in D_{A}(1, \infty)$ the function $t \mapsto e^{t A} x$ belongs to $C_{\alpha}^{\alpha}((0, T] ; D(A))$, and that $\left\|t^{\alpha} A e^{t A} x\right\|_{D_{A}(\alpha, \infty)}$ is bounded. For $0<\varepsilon \leq r<t \leq T$ it holds

$$
\begin{aligned}
\left\|A\left(e^{t A} x-e^{r A} x\right)\right\| & =\left\|\int_{r}^{t} A^{2} e^{\sigma A} x d \sigma\right\| \leq \frac{M_{2,1}}{\varepsilon^{\alpha}} \int_{r}^{t} \frac{d \sigma}{\sigma^{1-\alpha}}\|x\|_{D_{A}(1, \infty)} \\
& \leq \frac{M_{2,1}}{\alpha \varepsilon^{\alpha}}(t-r)^{\alpha}\|x\|_{D_{A}(1, \infty)}
\end{aligned}
$$

Moreover, estimate (3.2.4) yields

$$
\begin{aligned}
\left\|t^{\alpha} A e^{t A} x\right\|_{D_{A}(\alpha, \infty)} & =\left\|t^{\alpha} A e^{t A} x\right\|+\sup _{0<\xi \leq 1}\left\|t^{\alpha} \xi^{1-\alpha} A^{2} e^{(t+\xi) A} x\right\| \\
& \leq T^{\alpha} M_{1,1}\|x\|_{D_{A}(1, \infty)}+M_{2,1}\|x\|_{D_{A}(1, \infty)}, \quad 0<t \leq T .
\end{aligned}
$$

Statement (ii) follows now easily.

To prove statement (iii) we will show that $A u$ is continuous up to $t=0$, provided $f$ is continuous up to $t=0$ and $A u_{0}+f(0) \in \overline{D(A)}$. Then the statement will follow from Lemma 3.2.6.

We know already that $A u$ is continuous in $(0, T]$, so that we have only to prove that $A u(t) \rightarrow A u_{0}$ as $t \rightarrow 0$. Fix $\varepsilon>0$, and let $\delta \in(0,1)$ be such that

$$
\int_{1-\delta}^{1}(1-\sigma)^{\alpha-1} \sigma^{-\alpha} d \sigma \leq \varepsilon
$$

Split again $u(t)$ as $u(t)=u_{1}(t)+u_{2}(t)$, where $u_{1}$ and $u_{2}$ are defined by (3.2.20). If $t$ is so small that

$$
\sup _{0 \leq s \leq t}\|f(s)-f(t)\| \leq \varepsilon|\log \delta|^{-1}
$$

then

$$
\begin{aligned}
\left\|A u_{1}(t)\right\| \leq & \left\|\int_{0}^{t(1-\delta)} A e^{(t-s) A}(f(s)-f(t)) d s\right\| \\
& +\left\|\int_{t(1-\delta)}^{t} A e^{(t-s) A}(f(s)-f(t)) d s\right\| \\
\leq & M_{1}|\log \delta| \sup _{0 \leq s \leq t}\|f(s)-f(t)\|+M_{1} \int_{1-\delta}^{1}(1-\sigma)^{\alpha-1} \sigma^{-\alpha} d \sigma[f]_{C_{\alpha}^{\alpha}(x)} \\
\leq & M_{1} \varepsilon\left(1+[f]_{C_{\alpha}^{\alpha}(X)}\right) .
\end{aligned}
$$


Therefore, $A u_{1}(t) \rightarrow 0$ as $t \rightarrow 0$. Moreover, by (3.2.26) it follows easily that $A u_{2}(t) \rightarrow$ $A u_{0}$ as $t \rightarrow 0$. Hence, $A u$ is continuous up to $t=0$.

Now we consider the case where $f$ is unbounded near $t=0$. The introduction of the weighted spaces $C_{\alpha+\mu}^{\alpha}((0, T] ; X)$ and $C_{\alpha+\mu}^{\alpha}((0, T] ; D(A))$ is motivated again by the behavior of $e^{t A} x$ as $t \rightarrow 0$ : indeed, it is easy to see that if $x \in D_{A}(1-\mu, \infty), 0<\mu<1$, then $t \mapsto e^{t A} x$ belongs to $C_{\alpha+\mu}^{\alpha}((0, T] ; D(A))$ for each $\alpha \in(0,1)$. We recall that $f \in$ $C_{\alpha+\mu}^{\alpha}((0, T] ; X)$ means that $t \mapsto t^{\mu} f(t)$ is bounded and $t \mapsto t^{\alpha+\mu} f(t)$ is uniformly $\alpha$ Hölder continuous in $(0, T]$ with values in $X$. Of course, since $f$ is possibly unbounded, we will not get a strict solution but only a classical one.

Theorem 3.2.19. Let $0<\alpha, \mu<1, f \in C_{\alpha+\mu}^{\alpha}((0, T] ; X)$. Then $v=e^{t A} * f$ belongs to $C_{\alpha+\mu}^{\alpha}((0, T] ; D(A))$, it is differentiable in $(0, T]$ with values in $X, v^{\prime} \in C_{\alpha+\mu}^{\alpha}((0, T] ; X) \cap$ $B_{\alpha+\mu}\left((0, T] ; D_{A}(\alpha, \infty)\right)$, and there is $C$ such that

$$
\|v\|_{C_{\alpha+\mu}^{\alpha}(D(A))}+\left\|v^{\prime}\right\|_{C_{\alpha+\mu}^{\alpha}(X)}+\left\|v^{\prime}\right\|_{B_{\alpha+\mu}\left(D_{A}(\alpha, \infty)\right)} \leq C\|f\|_{C_{\alpha+\mu}^{\alpha}(X)} .
$$

Consequently,

(i) if $u_{0} \in \overline{D(A)}$, then the mild solution $u$ of (3.2.1) is classical;

(ii) if $u_{0} \in D_{A}(1-\mu, \infty)$, then $u^{\prime}$ and $A u$ belong to $C_{\alpha+\mu}^{\alpha}((0, T] ; X)$, $u^{\prime}$ belongs to $B_{\alpha+\mu}\left((0, T], D_{A}(\alpha, \infty)\right)$, and there is $C$ such that

$$
\begin{aligned}
& \|u\|_{C_{\alpha+\mu}^{\alpha}(D(A))}+\left\|u^{\prime}\right\|_{C_{\alpha+\mu}^{\alpha}(X)}+\left\|u^{\prime}\right\|_{B_{\alpha+\mu}\left(D_{A}(\alpha, \infty)\right)} \\
& \leq C\left(\left\|u_{0}\right\|_{D_{A}(1-\mu, \infty)}+\|f\|_{C_{\alpha+\mu}^{\alpha}(X)}\right)
\end{aligned}
$$

Proof $v$ belongs to $C((0, T] ; D(A)) \cap C^{1}((0, T] ; X)$ thanks to Theorem 3.2.16. To estimate $A v(t)$, we split it in three addenda, setting

$$
A v(t)=\int_{0}^{t / 2} A e^{(t-s) A} f(s) d s+\int_{t / 2}^{t} A e^{(t-s) A}(f(s)-f(t)) d s+\left(e^{t A / 2}-I\right) f(t),
$$

and we get

$$
\begin{aligned}
t^{\mu}\|A v(t)\| \leq & M_{1} t^{\mu} \int_{0}^{t / 2}(t-s)^{-1} s^{-\mu} d s\|f\|_{B_{\mu}(X)}+\left(M_{0}+1\right)\|f\|_{B_{\mu}(X)} \\
& +M_{1} t^{\mu} \int_{t / 2}^{t}(t-s)^{\alpha-1} s^{-\alpha-\mu} d s[f]_{C_{\alpha+\mu}^{\alpha}(X)} \\
\leq & M_{1} \int_{0}^{1 / 2}(1-\sigma)^{-1} \sigma^{-\mu} d \sigma\|f\|_{B_{\mu}(X)}+\left(M_{0}+1\right)\|f\|_{B_{\mu}(X)} \\
& +M_{1} \int_{1 / 2}^{1}(1-\sigma)^{\alpha-1} \sigma^{-\alpha-\mu} d \sigma[f]_{C_{\alpha+\mu}^{\alpha}(X) .}
\end{aligned}
$$


So, $A v$ belongs to $B_{\mu}((0, T] ; X)$. Now we estimate $\left[v^{\prime}\right]_{D_{A}(\alpha, \infty)}$. For $0<t \leq T$ and $0<\xi \leq 1$,

$$
\begin{aligned}
t^{\alpha+\mu}\left\|\xi^{1-\alpha} A e^{\xi A} v^{\prime}(t)\right\| \leq & t^{\alpha+\mu} \xi^{1-\alpha}\left\|\int_{0}^{t / 2} A^{2} e^{(t+\xi-s) A}(f(s)-f(t)) d s\right\| \\
& +t^{\alpha+\mu} \xi^{1-\alpha}\left\|\int_{t / 2}^{t} A^{2} e^{(t+\xi-s) A}(f(s)-f(t)) d s\right\| \\
& +t^{\alpha+\mu}\left\|\xi^{1-\alpha} A e^{(t+\xi) A} f(t)\right\| \\
= & I_{1}+I_{2}+I_{3},
\end{aligned}
$$

where

$$
\begin{aligned}
I_{1} \leq & t^{\alpha+\mu} \xi^{1-\alpha}\left\|\int_{0}^{t / 2} A^{2} e^{(t+\xi-s) A} f(s) d s\right\| \\
& +t^{\alpha+\mu} \xi^{1-\alpha}\left\|\left(A e^{(t+\xi) A}-A e^{(t / 2+\xi) A}\right) f(t)\right\| \\
\leq & M_{2} t^{\alpha+\mu} \xi^{1-\alpha} \int_{0}^{t / 2}(t+\xi-s)^{-2} s^{-\mu} d s\|f\|_{B_{\mu}(X)} \\
& +t^{\alpha+\mu} \xi^{1-\alpha}\left\|\left(A e^{(t+\xi) A}-A e^{(t / 2+\xi) A}\right) f(t)\right\| \\
\leq & M_{2} \int_{0}^{1 / 2}(1-\sigma)^{-2} \sigma^{-\mu} d \sigma\|f\|_{B_{\mu}(X)}+M_{2}\left(1+2^{\alpha}\right)\|f\|_{B_{\mu}(X)} ; \\
I_{2} \leq & M_{2} t^{\alpha+\mu} \xi^{1-\alpha} \int_{t / 2}^{t} \frac{(t-s)^{\alpha}}{s^{\alpha+\mu}(t+\xi-s)^{2}} d s[f]_{C_{\alpha+\mu}^{\alpha}(X)} \\
\leq & 2^{\alpha+\mu} M_{2} \int_{0}^{+\infty} \sigma^{\alpha}(\sigma+1)^{-2} d \sigma[f]_{C_{\alpha+\mu}^{\alpha}(X)} ; \\
I_{3} \leq & M_{1} t^{\alpha+\mu} \xi^{1-\alpha}(t+\xi)^{-1}\|f(t)\| \leq M_{1}\|f\|_{B_{\mu}(X) .} .
\end{aligned}
$$

To conclude the proof, it is sufficient to argue as we did in the proof of Theorem 3.2.17 and of Corollary 3.2.18(i)(ii).

\subsubsection{Space regularity}

We consider several regularity assumptions on $f$, which are suggested by the behavior of $t \mapsto e^{t A} u_{0}$ and of $t \mapsto d / d t e^{t A} u_{0}=A e^{t A} u_{0}$ as functions with values in $D_{A}(\alpha, \infty)$. To begin with, we consider the case where $u_{0} \in D_{A}(\alpha+1, \infty)$, then $A e^{t A} u_{0}$ is continuous for $t>0$ and bounded near $t=0$ with values in $D_{A}(\alpha, \infty)$. If in addition $u_{0} \in D_{A}(\alpha+1)$, then $t \mapsto A e^{t A} u_{0}$ is continuous up to $t=0$ with values in $D_{A}(\alpha)$. So, first we consider the case where $f$ is bounded with values in $D_{A}(\alpha, \infty)$, and it has some continuity property, at least for $t>0$. Then we consider the case of unbounded $f$.

The case where $f$ is bounded with values in $D_{A}(\alpha, \infty)$

Theorem 3.2.20. Let $0<\alpha<1$, and let $f \in C((0, T] ; X) \cap B\left((0, T] ; D_{A}(\alpha, \infty)\right)$. Then $v=e^{t A} * f$ has values in $D(A)$, it is differentiable for $t>0$ with values in $X$, 
and it is the classical solution of

$$
v^{\prime}(t)=A v(t)+f(t), \quad 0<t \leq T, \quad v(0)=0 .
$$

Moreover, $v^{\prime}$ and $A v$ belong to $C((0, T] ; X) \cap B\left((0, T] ; D_{A}(\alpha, \infty)\right)$, Av belongs to $B U C^{\alpha}$ $([0, T] ; X)$, and there is $C$ such that

$$
\left\|v^{\prime}\right\|_{B\left(D_{A}(\alpha, \infty)\right)}+\|A v\|_{B\left(D_{A}(\alpha, \infty)\right)}+\|A v\|_{B U C^{\alpha}(X)} \leq C\|f\|_{B\left(D_{A}(\alpha, \infty)\right)} .
$$

In addition,

(i) if $f \in C([0, T] ; X) \cap B\left([0, T] ; D_{A}(\alpha, \infty)\right)$, then $v^{\prime}$ and $A v$ are continuous with values in $X$ up to $t=0$, and $v$ is a strict solution of (3.2.34);

(ii) if $f \in C\left((0, T] ; D_{A}(\alpha, \infty)\right) \cap B\left([0, T] ; D_{A}(\alpha, \infty)\right)$, then $v \in C\left((0, T] ; D_{A}(\alpha+\right.$ $1, \infty)) \cap B\left([0, T] ; D_{A}(\alpha+1, \infty)\right) \cap C^{1}\left((0, T] ; D_{A}(\alpha, \infty)\right)$.

Proof Let us show that $v$ is a classical solution of (3.2.34), and that (3.2.35) holds. For $0 \leq t \leq T, v(t)$ belongs to $D(A)$, and

$$
\|A v(t)\| \leq M_{1, \alpha} \int_{0}^{t}(t-s)^{\alpha-1} d s\|f\|_{B\left(D_{A}(\alpha, \infty)\right)}=\frac{T^{\alpha} M_{1, \alpha}}{\alpha}\|f\|_{B\left(D_{A}(\alpha, \infty)\right)} .
$$

Moreover, for $0<\xi \leq 1$,

$$
\begin{aligned}
\left\|\xi^{1-\alpha} A e^{\xi A} A v(t)\right\| & =\xi^{1-\alpha}\left\|\int_{0}^{t} A^{2} e^{(t+\xi-s) A} f(s) d s\right\| \\
& \leq M_{2, \alpha} \xi^{1-\alpha} \int_{0}^{t}(t+\xi-s)^{\alpha-2} d s\|f\|_{B\left(D_{A}(\alpha, \infty)\right)} \\
& \leq \frac{M_{2, \alpha}}{1-\alpha}\|f\|_{B\left(D_{A}(\alpha, \infty)\right)},
\end{aligned}
$$

so that $A v$ is bounded with values in $D_{A}(\alpha, \infty)$.

Let us show that $A v$ is uniformly Hölder continuous with values in $X$ : for $0 \leq s<$ $t \leq T$, we have

$$
\begin{aligned}
& \|A v(t)-A v(s)\| \\
\leq & \left\|A \int_{0}^{s}\left(e^{(t-\sigma) A}-e^{(s-\sigma) A}\right) f(\sigma) d \sigma\right\|_{t}^{t}\left\|A \int_{s}^{t} e^{(t-\sigma) A} f(\sigma) d \sigma\right\| \\
\leq & \left(M_{2, \alpha} \int_{0}^{s} d \sigma \int_{s-\sigma}^{t-\sigma} \tau^{\alpha-2} d \tau+M_{1, \alpha} \int_{s}^{t}(t-\sigma)^{\alpha-1} d \sigma\right)\|f\|_{B\left(D_{A}(\alpha, \infty)\right)} \\
\leq & \left(\frac{M_{2, \alpha}}{\alpha(1-\alpha)}+\frac{M_{1, \alpha}}{\alpha}\right)(t-s)^{\alpha}\|f\|_{B\left(D_{A}(\alpha, \infty)\right)},
\end{aligned}
$$

so that $A v$ is uniformly $\alpha$-Hölder continuous in $[0, T]$. Estimate (3.2.35) follows now from (3.2.36), (3.2.37), (3.2.38). Moreover, thanks to Lemma 3.2.6, $v$ is a classical solution of (3.2.34) if $f \in C((0, T] ; X)$, and it is a strict solution if $f \in C([0, T] ; X)$. 
Let us prove statement (ii). Let $f \in C\left((0, T] ; D_{A}(\alpha, \infty)\right) \cap B\left([0, T], D_{A}(\alpha, \infty)\right)$. We are going to show that $A v$ belongs to $C\left([a, T] ; D_{A}(\alpha, \infty)\right)$ for every $a \in(0, T)$. For $\varepsilon>0$, let $\delta \in(0, \varepsilon \wedge a]$ be such that for $t_{1}, t_{2} \in[a, T],\left|t_{1}-t_{2}\right| \leq \delta$, we have $\left\|f\left(t_{1}\right)-f\left(t_{2}\right)\right\|_{D_{A}(\alpha, \infty)} \leq \varepsilon$, here $\varepsilon \wedge a:=\min \{\varepsilon, a\}$. Then for $a \leq r<t \leq T, t-r \leq \delta$, and for $0<\xi \leq 1$

$$
\begin{aligned}
& \left\|\xi^{1-\alpha} A e^{\xi A}(A v(t)-A v(r))\right\| \\
\leq & \left\|\xi^{1-\alpha} A^{2} \int_{r-\delta}^{r} e^{(\xi+s) A}[f(t-s)-f(r-s)] d s\right\| \\
& +\left\|\xi^{1-\alpha} A^{2} \int_{0}^{r-\delta} e^{(\xi+s) A}[f(t-s)-f(r-s)] d s\right\| \\
& +\left\|\xi^{1-\alpha} A^{2} \int_{r}^{t} e^{(\xi+s) A} f(t-s) d s\right\| \\
\leq & \frac{M_{2, \alpha}}{1-\alpha} \xi^{1-\alpha}\left(\xi^{-1+\alpha}-(\xi+r-\delta)^{-1+\alpha}\right) \varepsilon \\
& +\frac{M_{2, \alpha}}{1-\alpha} \xi^{1-\alpha}\left((\xi+r-\delta)^{-1+\alpha}-(\xi+r)^{-1+\alpha}\right) 2\|f\|_{B\left([0, T] ; D_{A}(\alpha, \infty)\right)} \\
& +\frac{M_{2, \alpha}}{1-\alpha} \xi^{1-\alpha}\left((\xi+r)^{-1+\alpha}-(\xi+t)^{-1+\alpha}\right)\|f\|_{B\left([0, T] ; D_{A}(\alpha, \infty)\right)} \\
\leq & \frac{M_{2, \alpha}}{1-\alpha}\left(\varepsilon+3\|f\|_{B\left([0, T] ; D_{A}(\alpha, \infty)\right)} \frac{\varepsilon^{1-\alpha}}{a^{1-\alpha}}\right) .
\end{aligned}
$$

Taking the supremum over $\xi \in(0,1]$, we see that $[A v(t)-A v(r)]_{D_{A}(\alpha, \infty)}$ goes to 0 as $t-r$ goes to 0 . Since $A v$ is continuous with values in $X$, then $v \in C\left((0, T] ; D_{A}(\alpha+1, \infty)\right)$. From the equality $v^{\prime}=A v+f$ it follows that $v^{\prime}$ is continuous in $(0, T]$ with values in $D_{A}(\alpha, \infty)$, and hence that $v$ is continuously differentiable in $(0, T]$ with values in $D_{A}(\alpha, \infty)$.

Corollary 3.2.21. Let $0<\alpha<1, u_{0} \in X, f \in C((0, T] ; X) \cap B\left((0, T] ; D_{A}(\alpha, \infty)\right)$, and let $u$ be the mild solution of (3.2.1). Then $u \in C^{1}((0, T] ; X) \cap C((0, T] ; D(A))$, and $u \in B\left([\varepsilon, T] ; D_{A}(\alpha, \infty)\right)$ for every $\varepsilon \in(0, T)$. Moreover, the following statements hold.

(i) if $u_{0} \in \overline{D(A)}$, then $u$ is a classical solution;

(ii) if $u_{0} \in D(A), A u_{0} \in \overline{D(A)}$, and $f \in C([0, T] ; X) \cap B\left([0, T] ; D_{A}(\alpha, \infty)\right)$, then $u$ is a strict solution;

(iii) if $u_{0} \in D_{A}(\alpha+1, \infty)$ and $f \in C([0, T] ; X) \cap B\left([0, T] ; D_{A}(\alpha, \infty)\right)$, then $u^{\prime}$ and $A u$ belong to $C([0, T] ; X) \cap B\left([0, T] ; D_{A}(\alpha, \infty)\right)$, Au belongs to $B U C^{\alpha}([0, T] ; X)$, and there is $C$ such that

$$
\begin{gathered}
\left\|u^{\prime}\right\|_{B\left(D_{A}(\alpha, \infty)\right)}+\|A u\|_{B\left(D_{A}(\alpha, \infty)\right)}+\|A u\|_{B U C^{\alpha}(X)} \\
\leq C\left(\|f\|_{B\left(D_{A}(\alpha, \infty)\right)}+\left\|u_{0}\right\|_{D_{A}(\alpha+1, \infty)}\right)
\end{gathered}
$$

(iv) if $u_{0} \in D_{A}(\alpha+1, \infty)$ and $f \in C\left((0, T] ; D_{A}(\alpha, \infty)\right)$, then $u \in C^{1}\left((0, T] ; D_{A}(\alpha, \infty)\right)$ $\cap C\left((0, T] ; D_{A}(\alpha+1, \infty)\right)$. 
Corollary 3.2.22. Let $0<\alpha<1$, and let $f \in C\left([0, T] ; D_{A}(\alpha)\right), u_{0} \in D_{A}(\alpha+1)$. Then the solution $u$ of problem (3.2.1) belongs to $C\left([0, T] ; D_{A}(\alpha+1)\right) \cap C^{1}\left([0, T] ; D_{A}(\alpha)\right)$, and $u^{\prime}$ belongs to buc ${ }^{\alpha}([0, T] ; X)$.

The case where $f$ is unbounded with values in $D_{A}(\alpha, \infty)$

In the next theorem we show that problem (3.2.1) may have a classical solution even if $f$ is unbounded with values in $D_{A}(\alpha, \infty)$ near $t=0$.

Theorem 3.2.23. Let $0<\alpha<1, f \in L_{1}(0, T ; X) \cap C((0, T] ; X) \cap B\left([\varepsilon, T] ; D_{A}(\alpha, \infty)\right)$ for every $\varepsilon \in(0, T)$, and let $u_{0} \in X$. Then the mild solution $u$ of problem (3.2.1) belongs to $C((0, T] ; D(A)) \cap C^{1}((0, T] ; X) \cap B\left([\varepsilon, T] ; D_{A}(\alpha+1, \infty)\right)$, for every $\varepsilon \in(0, T)$. If in addition, $u_{0} \in \overline{D(A)}$, then $u$ is a classical solution.

If $f$ belongs also to $C\left((0, T] ; D_{A}(\alpha, \infty)\right)$ (respectively, to $\left.C\left((0, T] ; D_{A}(\alpha)\right)\right)$, then $v^{\prime}$ and $A v$ belong to $C\left((0, T] ; D_{A}(\alpha, \infty)\right.$ ) (respectively, to $C\left((0, T] ; D_{A}(\alpha)\right)$ ).

Proof The proof is similar to the proof of Theorem 3.2.16. We split $u$ as $u(t)=$ $e^{t A} u_{0}+v(t)$, where $v=e^{t A} * f$. The first addendum is obviously continuous in $(0, T]$ with values in $D(A)$, and it belongs to $C([0, T] ; X)$ if and only if $u_{0} \in \overline{D(A)}$.

Let us consider the function $v$. By Proposition 3.2.10, $v$ is continuous in $[0, T]$ with values in $X$. Moreover, for $0<\varepsilon \leq t \leq T$,

$$
v(t)=e^{(t-\varepsilon) A} v(\varepsilon)+\int_{\varepsilon}^{t} e^{(t-s) A} f(s) d s=v_{1}(t)+v_{2}(t),
$$

where $v_{1}$ belongs to $C^{\infty}\left((\varepsilon, T] ; D\left(A^{n}\right)\right)$ for every $n$, and $v_{2}$ belongs to $C([\varepsilon, T] ; D(A)) \cap$ $C^{1}([\varepsilon, T] ; X) \cap B\left([\varepsilon, T] ; D_{A}(\alpha+1, \infty)\right)$, thanks to Theorem 3.2.20. Since $\varepsilon$ is arbitrary, then $u \in C((0, T] ; D(A)) \cap C^{1}((0, T] ; X)$.

If in addition $u_{0} \in \overline{D(A)}$, then $u \in C([0, T] ; X)$, so that it is a classical solution thanks to Lemma 3.2.6.

Let now $f$ be continuous in $(0, T]$ with values in $D_{A}(\alpha, \infty)$ (respectively, $D_{A}(\alpha)$ ). For $0<\varepsilon \leq t \leq T$, split $v(t)=v_{1}(t)+v_{2}(t)$ as above. Then $v_{1}$ belongs to $C^{\infty}\left((\varepsilon, T] ; D\left(A^{n}\right)\right)$ for every $n \in \mathbb{N}$, and $v_{2}$ belongs to $C\left([\varepsilon, T] ; D_{A}(\alpha+1, \infty)\right)$ (respectively, to $\left.C\left([\varepsilon, T] ; D_{A}(\alpha+1)\right)\right)$ thanks to Theorem 3.2.20(ii) (respectively, to Corollary 3.2.22) applied in the interval $[\varepsilon, T]$. Since $\varepsilon$ is arbitrary, then $v^{\prime}$ and $A v$ belong to $C\left((0, T] ; D_{A}(\alpha, \infty)\right)$ (respectively, to $\left.C\left((0, T] ; D_{A}(\alpha)\right)\right)$.

If we know how $\|f(t)\|_{D_{A}(\alpha, \infty)}$ blows up as $t \rightarrow 0$, we may give precise information on the behavior of $u(t)$ as $t \rightarrow 0$, getting also optimal regularity results. In the next theorem we consider the case where $f$ belongs to $B_{\theta}\left((0, T] ; D_{A}(\alpha, \infty)\right)$, that is $\left\|t^{\theta} f(t)\right\|_{D_{A}(\alpha, \infty)}$ is bounded, for some $\theta \in(0,1)$.

Theorem 3.2.24. Let $0<\alpha, \theta<1$. and let $f \in C((0, T] ; X) \cap B_{\theta}\left((0, T] ; D_{A}(\alpha, \infty)\right)$. Then $v=e^{t A} * f$ has values in $D(A)$, it is differentiable with values in $X$ for $t>0$, and it is a classical solution of (3.2.34). Moreover, $v^{\prime}$ and $A v$ belong to $C((0, T] ; X) \cap$ $B_{\theta}\left((0, T] ; D_{A}(\alpha, \infty)\right)$, Av belongs to $C_{\theta}^{\alpha}((0, T] ; X)$, and there is $C$ such that

$$
\left\|v^{\prime}\right\|_{B_{\theta}\left(D_{A}(\alpha, \infty)\right)}+\|A v\|_{B_{\theta}\left(D_{A}(\alpha, \infty)\right)}+\|A v\|_{C_{\theta}^{\alpha}(X)} \leq C\|f\|_{B_{\theta}\left(D_{A}(\alpha, \infty)\right)} .
$$


In particular, if $\theta=\alpha$ then $v$ is bounded with values in $D(A)$. If $\theta<\alpha$, then Av belongs to $B U C^{\alpha-\theta}([0, T] ; X)$, and there is $C$ such that

$$
\|A v\|_{B U C^{\alpha-\theta}(X)} \leq C\|f\|_{\left.B_{\theta}\left(D_{A}(\alpha, \infty)\right)\right)} .
$$

If $f$ belongs also to $C\left((0, T] ; D_{A}(\alpha, \infty)\right)$ (respectively, to $C\left((0, T] ; D_{A}(\alpha)\right)$ ), then $v^{\prime}$ and Av belong to $C\left((0, T] ; D_{A}(\alpha, \infty)\right)$ (respectively, to $C\left((0, T] ; D_{A}(\alpha)\right)$ ).

Proof By Theorem 3.2.23, $v$ is continuous in $(0, T]$ with values in $D(A)$, and continuously differentiable in $(0, T]$ with values in $X$. Moreover,

$$
\begin{aligned}
\|A v(t)\| & \leq M_{1, \alpha} \int_{0}^{t}(t-s)^{\alpha-1} s^{-\theta} d s\|f\|_{B_{\theta}\left(D_{A}(\alpha, \infty)\right)} \\
& =M_{1, \alpha} t^{\alpha-\theta} \int_{0}^{1}(1-\sigma)^{\alpha-1} \sigma^{-\theta} d \sigma\|f\|_{B_{\theta}\left(D_{A}(\alpha, \infty)\right)} \\
& \leq K t^{\alpha-\theta}\|f\|_{B_{\theta}\left(D_{A}(\alpha, \infty)\right)} .
\end{aligned}
$$

Fix now any $r \in(0, T)$. For $t \geq r$, it holds

$$
A v(t)=A e^{(t-r / 2) A} v(r / 2)+\int_{r / 2}^{t} A e^{(t-s) A} f(s) d s=A v_{1}(t)+A v_{2}(t) .
$$

Denoting by $C_{\alpha}$ the norm of the embedding $D_{A}(\alpha, 1) \subset D_{A}(\alpha, \infty)$ and using (3.2.5), we get

$$
\begin{aligned}
& \left\|A v_{1}(t)\right\|_{D_{A}(\alpha, \infty)} \leq \frac{K_{0, \alpha} C_{\alpha}}{(t-r / 2)^{\alpha}}\|A v(r / 2)\| \\
& \leq \frac{K_{0, \alpha} C_{\alpha}}{(r / 2)^{\alpha}} K(r / 2)^{\alpha-\theta}\|f\|_{B_{\theta}\left(D_{A}(\alpha, \infty)\right)}=\frac{K_{0, \alpha} C_{\alpha} K}{(r / 2)^{\theta}}\|f\|_{B_{\theta}\left(D_{A}(\alpha, \infty)\right) .}
\end{aligned}
$$

Moreover, from Theorem 3.2.20 applied in the interval $[r / 2, T]$, we get for every $t \in$ $[r / 2, T]$

$$
\left\|A v_{2}(t)\right\|_{D_{A}(\alpha, \infty)} \leq C \sup _{r / 2 \leq s \leq T}\|f(s)\|_{D_{A}(\alpha, \infty)} \leq \frac{C}{(r / 2)^{\theta}}\|f\|_{B_{\theta}\left(D_{A}(\alpha, \infty)\right)} .
$$

Summing up and taking $t=r$ we see that

$$
\left\|r^{\theta} A v(r)\right\|_{D_{A}(\alpha, \infty)} \leq\left(2^{\theta} K_{0, \alpha} C_{\alpha} K+2^{\theta} C\right)\|f\|_{B_{\theta}\left(D_{A}(\alpha, \infty)\right)},
$$

which implies that $A v \in B_{\theta}\left((0, T] ; D_{A}(\alpha, \infty)\right)$.

The proof of the statements about the Hölder continuity of $A v$ is similar to the proof of statements (ii)-(iii) of Proposition 3.2.10. We have seen above that $v(r / 2)$ belongs to $D(A)$. Moreover, we have seen in the proof of Corollary 3.2.18 that for every $x \in D(A)$ the function $t \mapsto A e^{(t-r / 2) A} x$ belongs to $C_{\alpha}^{\alpha}((r / 2, T] ; X)$, and its $C_{\alpha}^{\alpha}$-norm is less or equal to $C\|A x\|$, for some $C>0$. Using estimate (3.2.42) we get

$$
\left\|A v_{1}\right\|_{B U C^{\alpha}([r, T] ; X)} \leq C(r / 2)^{-\alpha}\|A v(r / 2)\| \leq C K(r / 2)^{-\theta}\|f\|_{B_{\theta}\left(D_{A}(\alpha, \infty)\right)} .
$$


Due again to Theorem 3.2.20, $A v_{2}$ belongs to $B U C^{\alpha}([r / 2, T] ; X)$, and that

$$
\left\|A v_{2}\right\|_{B U C^{\alpha}([r / 2, T] ; X)} \leq C\|f\|_{B\left([r / 2, T] ; D_{A}(\alpha, \infty)\right)} \leq C(r / 2)^{-\theta}\|f\|_{B_{\theta}\left(D_{A}(\alpha, \infty)\right)} .
$$

Summing up we get

$$
\|A v\|_{B U C^{\alpha}([r, T] ; X)} \leq \text { const. } r^{-\theta}\|f\|_{B_{\theta}\left(D_{A}(\alpha, \infty)\right)},
$$

which implies that $A v$ belongs to $C_{\theta}^{\alpha}((0, T] ; X)$. Estimate (3.2.40) follows now easily.

Let us prove that if $\theta<\alpha$ then $A v \in B U C^{\alpha-\theta}([0, T] ; X)$. For $0 \leq r<t \leq T$, we have

$$
\begin{aligned}
& \|A v(t)-A v(r)\| \leq\left\|\int_{0}^{r}\left(\int_{r-s}^{t-s} A^{2} e^{\sigma A} d \sigma\right) f(s) d s\right\|+\left\|\int_{r}^{t} A e^{(t-s) A} f(s) d s\right\| \\
& \leq\left(M_{2, \alpha} \int_{0}^{r} s^{-\theta} \int_{r-s}^{t-s} \frac{1}{\sigma^{2-\alpha}} d \sigma d s+M_{1, \alpha} \int_{r}^{t} \frac{1}{(t-s)^{1-\alpha} s^{\theta}} d s\right)\|f\|_{B_{\theta}\left(D_{A}(\alpha, \infty)\right)}
\end{aligned}
$$

Following the proof of statement (iii) of Proposition 3.2.10, we find

$$
\begin{aligned}
& \|A v(t)-A v(r)\| \leq \frac{1}{\alpha-\theta}\left(M_{2, \alpha} \int_{0}^{1} \frac{d \sigma}{(1-\sigma)^{1-\theta} \sigma^{\theta}}\right. \\
& \left.+M_{1, \alpha} \int_{0}^{1} \frac{d \sigma}{(1-\sigma)^{1-\alpha} \sigma^{\theta}}\right)(t-r)^{\alpha-\theta}\|f\|_{B_{\theta}\left(D_{A}(\alpha, \infty)\right)}
\end{aligned}
$$

and estimate (3.2.41) follows.

The last statement follows from Theorem 3.2.23.

Corollary 3.2.25. Let $0<\alpha<1, u_{0} \in X, f \in C((0, T] ; X) \cap B_{\theta}\left((0, T] ; D_{A}(\alpha, \infty)\right)$, and let $u$ be the mild solution of problem (3.2.1). The following statements hold.

(i) If $u_{0} \in \overline{D(A)}$, then $u$ is a classical solution.

(ii) If $u_{0} \in D_{A}(\alpha+1-\theta, \infty)$, then $u^{\prime}$ and $A u$ belong to $B_{\theta}\left((0, T] ; D_{A}(\alpha, \infty)\right)$, Au belongs to $C_{\theta}^{\alpha}((0, T] ; X)$, and there is $C$ such that

$$
\begin{gathered}
\left\|u^{\prime}\right\|_{B_{\theta}\left(D_{A}(\alpha, \infty)\right)}+\|A u\|_{B_{\theta}\left(D_{A}(\alpha, \infty)\right)}+\|A u\|_{C_{\theta}^{\alpha}(X)} \\
\leq C\left(\|f\|_{B_{\theta}\left(D_{A}(\alpha, \infty)\right)}+\left\|u_{0}\right\|_{D_{A}(\alpha+1-\theta, \infty)}\right)
\end{gathered}
$$

Moreover, in the case where $\theta<\alpha$, then $u \in B U C^{\alpha-\theta}([0, T] ; D(A))$, and there is $C$ such that

$$
\|A u\|_{B U C^{\alpha-\theta}(X)} \leq C\left(\|f\|_{B_{\theta}\left(D_{A}(\alpha, \infty)\right)}+\left\|u_{0}\right\|_{D_{A}(\alpha+1-\theta, \infty)}\right)
$$

(iii) If in addition $f \in C\left((0, T] ; D_{A}(\alpha, \infty)\right)$ (respectively, $\left.f \in C\left((0, T] ; D_{A}(\alpha)\right)\right)$, then $u^{\prime}$, Au belong to $C\left((0, T] ; D_{A}(\alpha, \infty)\right)$ (respectively, to $\left.C\left((0, T] ; D_{A}(\alpha)\right)\right)$. 
(iv) In the case $\theta=\alpha$, if $u_{0} \in D(A), A u_{0} \in \overline{D(A)}$, and $f \in C([0, T] ; X) \cap B_{\alpha}((0, T]$; $\left.D_{A}(\alpha, \infty)\right), \lim _{t \rightarrow 0}\left\|t^{\alpha} f(t)\right\|_{D_{A}(\alpha, \infty)}=0$, then $u$ is a strict solution and

$$
\lim _{t \rightarrow 0}\left\|t^{\alpha} A u(t)\right\|_{D_{A}(\alpha, \infty)}=\lim _{t \rightarrow 0}\left\|t^{\alpha} u^{\prime}(t)\right\|_{D_{A}(\alpha, \infty)}=0 .
$$

Theorem 3.2.26. Let $0<\alpha, \mu<1$, and let $f \in C((0, T] ; X) \cap B_{\mu}((0, T] ; X) \cap$ $B_{\alpha+\mu}\left((0, T] ; D_{A}(\alpha, \infty)\right)$. Then $v=e^{t A} * f$ is a classical solution of (3.2.34), $v^{\prime}, A v$ belong to $B_{\mu}((0, T] ; X) \cap B_{\alpha+\mu}\left((0, T] ; D_{A}(\alpha, \infty)\right)$, Av belongs to $C_{\alpha+\mu}^{\alpha}((0, T] ; X)$, and there is $C$ such that

$$
\begin{aligned}
& \left\|v^{\prime}\right\|_{B_{\mu}(X)}+\left\|v^{\prime}\right\|_{B_{\alpha+\mu}\left(D_{A}(\alpha, \infty)\right)}+\|A v\|_{B_{\alpha+\mu}\left(D_{A}(\alpha, \infty)\right)} \\
& +\|A v\|_{C_{\alpha+\mu}^{\alpha}(X)} \leq C\left(\|f\|_{B_{\mu}(X)}+\|f\|_{B_{\alpha+\mu}\left(D_{A}(\alpha, \infty)\right)}\right) .
\end{aligned}
$$

If $f$ belongs also to $C\left((0, T] ; D_{A}(\alpha, \infty)\right)$ (respectively, to $\left.C\left((0, T] ; D_{A}(\alpha)\right)\right)$, then $v^{\prime}$ and Av belong to $C\left((0, T] ; D_{A}(\alpha, \infty)\right)$ (respectively, to $\left.C\left((0, T] ; D_{A}(\alpha)\right)\right)$.

Proof By Theorem 3.2.23, $v$ is continuous in $(0, T]$ with values in $D(A)$. Let us prove that $A v$ belongs to $B_{\mu}((0, T] ; X)$. For $0<t \leq T$, we have

$$
\left\|t^{\mu} A v(t)\right\| \leq t^{\mu}\left\|\int_{0}^{t / 2} A e^{(t-s) A} f(s) d s\right\|+t^{\mu}\left\|\int_{t / 2}^{t} A e^{(t-s) A} f(s) d s\right\|,
$$

so that

$$
\begin{aligned}
\left\|t^{\mu} A v(t)\right\| \leq & M_{1} t^{\mu} \int_{0}^{t / 2}(t-s)^{-1} s^{-\mu} d s\|f\|_{B_{\mu}(X)} \\
& +2^{\mu} M_{1, \alpha} \int_{t / 2}^{t}(t-s)^{\alpha-1} s^{-\alpha} d s\|f\|_{B_{\alpha+\mu}\left(D_{A}(\alpha, \infty)\right)} \\
\leq & M_{1} \int_{0}^{1 / 2}(1-\sigma)^{-1} \sigma^{-\mu} d \sigma\|f\|_{B_{\mu}(X)} \\
& +2^{\mu} M_{1, \alpha} \int_{1 / 2}^{1}(1-\sigma)^{\alpha-1} \sigma^{-\alpha} d \sigma\|f\|_{B_{\alpha+\mu}\left(D_{A}(\alpha, \infty)\right)} .
\end{aligned}
$$

From now on, the proof is similar to the proof of Theorem 3.2.24.

Corollary 3.2.27. Let $0<\alpha, \mu<1, u_{0} \in X, f \in C((0, T] ; X) \cap B_{\mu}((0, T] ; X) \cap$ $B_{\alpha+\mu}\left((0, T] ; D_{A}(\alpha, \infty)\right)$, and let $u$ be the mild solution of problem (3.2.1). Then

(i) if $u_{0} \in \overline{D(A)}$, then $u$ is a classical solution;

(ii) if $u_{0} \in D_{A}(1-\mu, \infty)$, then $u^{\prime}$ and $A u$ belong to $B_{\mu}((0, T] ; X) \cap B_{\alpha+\mu}((0, T]$; $\left.D_{A}(\alpha, \infty)\right)$, Au belongs to $C_{\alpha+\mu}^{\alpha}((0, T] ; X)$, and there is $C>0$ such that

$$
\begin{gathered}
\left\|u^{\prime}\right\|_{B_{\mu}(X)}+\left\|u^{\prime}\right\|_{B_{\alpha+\mu}\left(D_{A}(\alpha, \infty)\right)}+\|A u\|_{B_{\alpha+\mu}\left(D_{A}(\alpha, \infty)\right)}+\|A u\|_{C_{\alpha+\mu}^{\alpha}(X)} \\
\leq C\left(\|f\|_{B_{\mu}(X)}+\|f\|_{B_{\alpha+\mu}\left(D_{A}(\alpha, \infty)\right)}+\left\|u_{0}\right\|_{D_{A}(1-\mu, \infty)}\right)
\end{gathered}
$$




\subsubsection{A further regularity result}

Theorem 3.2.28. Let $0<\theta, \beta<1$, with $\theta+\beta \neq 1$. Let

$$
f \in B U C^{\theta}\left([0, T] ; D_{A}(\beta, \infty)\right), u_{0} \in D(A), A u_{0}+f(0) \in D_{A}(\theta+\beta, \infty) .
$$

Then the mild solution $u$ of problem (3.2.1) is strict, $u^{\prime}$ and $A u$ belong to $B U C^{\theta}([0, T]$; $\left.D_{A}(\beta, \infty)\right), u^{\prime} \in B\left([0, T] ; D_{A}(\theta+\beta, \infty)\right), A u \in B U C^{\theta+\beta}([0, T] ; X)$, and there exists $C>0$ such that

$$
\begin{gathered}
\|u\|_{B U C^{\theta+1}\left(D_{A}(\beta, \infty)\right)}+\|A u\|_{B U C^{\theta}\left(D_{A}(\beta, \infty)\right)}+\left\|u^{\prime}\right\|_{B\left(D_{A}(\theta+\beta, \infty)\right)}+\|A u\|_{B U C^{\theta+\beta}(X)} \\
\leq C\left(\|f\|_{B U C^{\theta}\left(D_{A}(\beta, \infty)\right)}+\left\|u_{0}\right\|_{D(A)}+\left\|A u_{0}+f(0)\right\|_{D_{A}(\theta+\beta, \infty)}\right) .
\end{gathered}
$$

Proof Let us consider problem (3.2.1) as an evolution equation in the space $Y=$ $D_{A}(\beta, \infty)$. The domain of the part of $A$ in $Y$ is $D\left(A_{Y}\right)=D_{A}(\beta+1, \infty)$, and the Reiteration Theorem 2.4.19 yields

$$
\left(Y, D\left(A_{Y}\right)\right)_{\theta, \infty}=D_{A}(\theta+\beta, \infty) .
$$

The function $f$ is uniformly Hölder continuous with values in $Y$, moreover $u_{0}$ belongs to $D\left(A_{Y}\right)$ and $A u_{0}+f(0)$ belongs to $D_{A_{Y}}(\theta, \infty)=\left(Y, D\left(A_{Y}\right)\right)_{\theta, \infty}$. By Theorem 3.2.13(iii), the mild solution in $Y$ is strict and belongs to $B U C^{\theta+1}\left([0, T] ; D_{A}(\beta, \infty)\right)$, moreover $u^{\prime} \in B\left([0, T] ; D_{A}(\theta+\beta, \infty)\right)$.

It remains to show that $A u \in B U C^{\theta+\beta}([0, T] ; X)$. Thanks to $(3.2 .23)$,

$$
A u(t)=\int_{0}^{t} A e^{(t-s) A}(f(s)-f(t)) d s+\left(e^{t A}-1\right) f(t)+A e^{t A} u_{0} .
$$

In the case $\theta+\beta<1, A u(t)-A(r)$ may be split for $0 \leq r<t \leq T$ as

$$
\begin{aligned}
& A u(t)-A u(r)=\int_{0}^{r} A\left(e^{(t-s) A}-e^{(r-s) A}\right)(f(s)-f(r)) d s \\
& +\int_{r}^{t} A e^{(t-s) A}(f(s)-f(t)) d s+\left[\left(e^{(t-r) A}-I\right)(f(t)-f(r))\right] \\
& +\left[\left(e^{t A}-e^{r A}\right)(f(r)-f(0))\right]+\left[\left(e^{t A}-e^{r A}\right)\left(A u_{0}+f(0)\right)\right] \\
& =\sum_{k=1}^{5} I_{k} .
\end{aligned}
$$

Each addendum $I_{k}$ may be estimated using the methods of Subsection 3.2.4, getting finally

$$
\begin{aligned}
& \|A u(t)-A u(r)\| \leq\left(\frac{M_{2, \beta}}{(1-\beta-\theta)(\beta+\theta)}+\frac{2 M_{1, \beta}}{\beta+\theta}+\frac{M_{1, \beta}}{\beta}\right) \\
& \cdot(t-r)^{\beta+\theta}[f]_{\theta}^{D_{A}(\beta, \infty)}+\frac{M_{1, \beta+\theta}}{\beta+\theta}(t-r)^{\beta+\theta}\left\|A u_{0}+f(0)\right\|_{D_{A}(\theta+\beta, \infty)} .
\end{aligned}
$$

Therefore, $A u$ is uniformly $(\beta+\theta)$-Hölder continuous with values in $X$. 
Let us consider the case $\theta+\beta>1$. To prove that $A u$ belongs to $B U C^{\theta+\beta}([0, T] ; X)$, it is sufficient to show that $u^{\prime}$ belongs to $B U C^{\theta+\beta-1}([0, T] ; D(A))$. Indeed, in this case $A u^{\prime}$ is continuous, so that

$$
\left\|\frac{A u(t+h)-A u(t)}{h}-A u^{\prime}(t)\right\|=\left\|\frac{1}{h} \int_{t}^{t+h}\left(A u^{\prime}(s)-A u^{\prime}(t)\right) d s\right\| .
$$

Letting $h \rightarrow 0$, we find that $A u$ is differentiable and

$$
(A u)^{\prime}=A u^{\prime} \in B U C^{\theta+\beta-1}([0, T] ; X) .
$$

For $0 \leq t \leq T$, we have

$$
u^{\prime}(t)=\int_{0}^{t} A e^{(t-s) A}(f(s)-f(t)) d s+e^{t A}(f(t)-f(0))+e^{t A}\left(A u_{0}+f(0)\right),
$$

from which it follows that $u^{\prime}(t) \in D(A)$, and

$$
\left\|A u^{\prime}(t)\right\| \leq\left(\frac{M_{2, \beta}}{\theta+\beta-1}+M_{1, \beta}\right) T^{\theta+\beta-1}[f]_{\theta}^{D_{A}(\beta, \infty)}+M_{0}\left\|A\left(A u_{0}+f(0)\right)\right\| .
$$

Moreover, for $0 \leq r<t \leq T$,

$$
\begin{aligned}
A u^{\prime}(t)-A u^{\prime}(r)= & \int_{0}^{r} A^{2}\left(e^{(t-s) A}-e^{(r-s) A}\right)(f(s)-f(r)) d s \\
& +\int_{(t-r) A}^{t} A^{2} e^{(t-s) A}(f(s)-f(t)) d s+\left(A e^{t A}-A e^{r A}\right)(f(r)-f(0)) \\
& A e^{(t-r) A}(f(r))+A\left(e^{t A}-e^{r A}\right)\left(A u_{0}+f(0)\right) \\
= & \sum_{k=1}^{5} J_{k} .
\end{aligned}
$$

Again, each $J_{k}$ may be estimated arguing as in Subsection 3.2.4, getting

$$
\begin{aligned}
& \left\|A u^{\prime}(t)-A u^{\prime}(r)\right\| \leq\left(\frac{M_{3, \beta}}{(2-\beta-\theta)(\beta+\theta-1)}+\frac{2 M_{2, \beta}}{\beta+\theta-1}+M_{1, \beta}\right) \\
& \cdot(t-r)^{\beta+\theta-1}[f]_{\theta}^{D_{A}(\beta, \infty)}+\frac{M_{1, \beta+\theta-1}}{\beta+\theta-1}(t-r)^{\beta+\theta-1}\left\|A\left(A u_{0}+f(0)\right)\right\|_{D_{A}(\theta+\beta-1, \infty)} .
\end{aligned}
$$

Therefore, $A u^{\prime}$ is uniformly $(\theta+\beta-1)$-Hölder continuous, and the statement follows.

\subsection{Fully nonlinear equation}

We now apply the results of the previous section to study fully nonlinear equations. Again we follow Lunardi's [24] approach, but we impose the simplifying assumption that the domain $D$ of the linearization is dense in the basic Banach space $X$. This simplification is adapted to our application to moving boundaries porous media. 
Let $D$ be a Banach space endowed with the norm $\|\cdot\|_{D}$, continuously embedded and dense in $X$, and let $\mathcal{O}$ be an open set in $D$. We consider the initial value problem

$$
u^{\prime}(t)=F(t, u(t)), \quad t>0 ; \quad u(0)=u_{0},
$$

where $F:[0, T] \times \mathcal{O} \rightarrow X$ is a nonlinear operator, $T \in(0,+\infty)$, and $u_{0} \in \mathcal{O}$. The key assumption on problem (3.3.1) is

$$
\left\{\begin{array}{l}
\mathcal{O} \subset D \text { is open, } F \in B C^{2}([0, T] \times \mathcal{O} ; X) \text { and } \\
F_{v}(t, v) \in \mathcal{H}(D, X) \text { for all }(t, v) \in[0, T] \times \mathcal{O}
\end{array}\right.
$$

\subsubsection{Local existence, uniqueness and regularity}

We now state the main local existence theorem. It is convenient to choose an arbitrary initial time $t_{0} \in[0, T)$, and to consider the initial value problem:

$$
u^{\prime}(t)=F(t, u(t)), \quad t_{0}<t \leq t_{0}+\delta ; \quad u\left(t_{0}\right)=x_{0},
$$

with $\delta \in\left(0, T-t_{0}\right]$ and $x_{0} \in \mathcal{O}$. We shall find a local strict solution, that is a solution $u \in C\left(\left[t_{0}, t_{0}+\delta\right] ; D\right) \cap C^{1}\left(\left[t_{0}, t_{0}+\delta\right] ; X\right)$, with $\delta$ small, which in addition belongs to the weighted Hölder space $C_{\alpha}^{\alpha}\left(\left(t_{0}, t_{0}+\delta\right] ; D\right)$.

Theorem 3.3.1. Let $\alpha \in(0,1)$. Further, assume that (3.3.2) holds true. Fix $\bar{t} \in[0, T]$ and $\bar{u} \in \mathcal{O}$. Then there are $\delta=\delta(\bar{t}, \bar{u})>0, r=r(\bar{t}, \bar{u})>0$ such that

(i) for every $t_{0} \in[\bar{t}-r, \bar{t}+r] \cap[0, T]$, and $x_{0} \in \mathcal{O}$ such that $\left\|x_{0}-\bar{u}\right\|_{D} \leq r$, there is a strict solution $u \in C\left(\left[t_{0}, t_{0}+\delta\right] ; D\right) \cap C^{1}\left(\left[t_{0}, t_{0}+\delta\right] ; X\right)$ of (3.3.3) in $\left[t_{0}, t_{0}+\delta\right]$;

(ii) $u$ belongs to $C_{\alpha}^{\alpha}\left(\left(t_{0}, t_{0}+\delta\right] ; D\right)$, u' belongs to $B_{\alpha}\left(\left(t_{0}, t_{0}+\delta\right] ;(X, D)_{\alpha, \infty}\right)$, and in addition

$$
\lim _{\varepsilon \rightarrow 0} \varepsilon^{\alpha}[u]_{B U C^{\alpha}\left(\left[t_{0}+\varepsilon, t_{0}+2 \varepsilon\right] ; D\right)}=0 .
$$

Moreover, $u$ is the unique solution of (3.3.3) belonging to

$$
\bigcup_{0<\beta<1} C_{\beta}^{\beta}\left(\left(t_{0}, t_{0}+\delta\right] ; D\right) \cap C\left(\left[t_{0}, t_{0}+\delta\right] ; D\right) .
$$

Proof We look for a solution of (3.3.3) belonging to the metric space

$$
\begin{array}{r}
Y=\left\{u \in C_{\alpha}^{\alpha}\left(\left(t_{0}, t_{0}+\delta\right] ; D\right) \cap C\left(\left[t_{0}, t_{0}+\delta\right] ; D\right) ;\right. \\
\left.u\left(t_{0}\right)=x_{0},\|u(\cdot)-\bar{u}\|_{C_{\alpha}^{\alpha}\left(\left(t_{0}, t_{0}+\delta\right] ; D\right)} \leq \rho\right\},
\end{array}
$$

endowed with the distance of $C_{\alpha}^{\alpha}\left(\left(t_{0}, t_{0}+\delta\right] ; D\right)$, where $\delta<T-t_{0}$ and $\rho<\operatorname{dist}(\bar{u}, \partial \mathcal{O})$ are positive numbers to be chosen later. Clearly, $Y$ is a closed set in $C_{\alpha}^{\alpha}\left(\left(t_{0}, t_{0}+\delta\right] ; D\right)$. Set moreover

$$
A=F_{u}(\bar{t}, \bar{u})
$$


Since $D$ endowed with the graph norm of $A$ is a Banach space, the norms $\|\cdot\|_{D}$ and $\|\cdot\|_{D(A)}$ are equivalent. Then it is easy to see that, due to (3.3.2), for every $u \in Y$ the function $t \mapsto F(t, u(t))-A u(t)$ belongs to $C_{\alpha}^{\alpha}\left(\left(t_{0}, t_{0}+\delta\right] ; X\right) \cap C\left(\left[t_{0}, t_{0}+\delta\right] ; X\right)$. By (3.3.2), there exists $M>0$ such that for $(t, u) \in[0, T] \times \mathcal{O}$,

$$
F(t, u), F_{t}(t, u), F_{x}(t, u), F_{x t}(t, u) \text { and } F_{x x}(t, u) \text { are bounded by } M \text {. }
$$

Define a nonlinear operator $\Gamma$ on $Y$, by $\Gamma(u)=v$, where $v$ is the solution of

$$
v^{\prime}(t)=A v(t)+[F(t, u(t))-A u(t)], \quad t_{0}<t \leq t_{0}+\delta ; \quad v\left(t_{0}\right)=x_{0} .
$$

By Corollary 3.2.18 (iii), for every $u \in Y, \Gamma(u)$ belongs to $C_{\alpha}^{\alpha}\left(\left(t_{0}, t_{0}+\delta\right] ; D\right) \cap C\left(\left[t_{0}, t_{0}+\right.\right.$ $\delta] ; D)$. It is clear that a function $u \in Y$ is a solution of (3.3.3) if and only if it is a fixed point of $\Gamma$. We shall show that $\Gamma$ is a contraction and maps $Y$ into itself, provided $\delta, \rho,\left|t_{0}-\bar{t}\right|$, and $\left\|x_{0}-\bar{u}\right\|_{D}$ are suitably small. Let $C$ be the constant given by Corollary 3.2.18 (ii), and let $\gamma, \gamma_{\alpha} \geq 1$ be such that

$$
\left\{\begin{array}{l}
\gamma^{-1}\|y\|_{D} \leq\|y\|+\|A y\| \leq \gamma\|y\|_{D}, \quad \forall y \in D, \\
\gamma_{\alpha}^{-1}\|y\|_{(X, D)_{\alpha, \infty}} \leq\|y\|_{D_{A}(\alpha, \infty)} \leq \gamma_{\alpha}\|y\|_{(X, D)_{\alpha, \infty}}, \quad \forall y \in(X, D)_{\alpha, \infty} .
\end{array}\right.
$$

Obviously, $C, \gamma, \gamma_{\alpha}$ depend only on $\bar{t}$ and $\bar{u}$. For simplicity, if $B$ is any Banach space, we write as usual $C(B), C_{\alpha}^{\alpha}(B)$ for $C\left(\left[t_{0}, t_{0}+\delta\right] ; B\right), C_{\alpha}^{\alpha}\left(\left(t_{0}, t_{0}+\delta\right] ; B\right)$. For $v_{1}, v_{2} \in Y$, we have

$$
\begin{aligned}
& \left\|\Gamma\left(v_{1}\right)-\Gamma\left(v_{2}\right)\right\|_{C_{\alpha}^{\alpha}(D)} \leq \gamma\left\|\Gamma\left(v_{1}\right)-\Gamma\left(v_{2}\right)\right\|_{C_{\alpha}^{\alpha}(D(A))} \\
& \leq \gamma C\left\|F\left(\cdot, v_{1}(\cdot)\right)-F\left(\cdot, v_{2}(\cdot)\right)-A\left(v_{1}(\cdot)-v_{2}(\cdot)\right)\right\|_{C_{\alpha}^{\alpha}(X)} .
\end{aligned}
$$

For each $t \in\left[t_{0}, t_{0}+\delta\right]$, we have

$$
\begin{aligned}
& \left\|F\left(t, v_{1}(t)\right)-F\left(t, v_{2}(t)\right)-A\left(v_{1}(t)-v_{2}(t)\right)\right\| \\
\leq & \left\|\int_{0}^{1}\left[F_{x}\left(t, \sigma v_{1}(t)+(1-\sigma) v_{2}(t)\right)-A\right] d \sigma\left(v_{1}(t)-v_{2}(t)\right)\right\| \\
\leq & \int_{0}^{1}\left\|F_{x}\left(t, \sigma v_{1}(t)+(1-\sigma) v_{2}(t)\right)-A\right\|_{\mathcal{L}(D, X)} d \sigma\left\|v_{1}(t)-v_{2}(t)\right\|_{D} \\
\leq & \left(\left\|F_{x t}\right\|_{\infty}|t-\bar{t}|+\left\|F_{x x}\right\|_{\infty} \rho\right)\left\|v_{1}(t)-v_{2}(t)\right\|_{D} \\
\leq & M\left(\left|t_{0}-\bar{t}\right|+\delta+\rho\right)\left\|v_{1}(t)-v_{2}(t)\right\|_{D},
\end{aligned}
$$

and for $t_{0}+\varepsilon \leq s<t \leq t_{0}+\delta$,

$$
\begin{aligned}
& \| F\left(t, v_{1}(t)\right)-F\left(t, v_{2}(t)\right)-F\left(s, v_{1}(s)\right)+F\left(s, v_{2}(s)\right) \\
& -A\left(v_{1}(t)-v_{2}(t)-v_{1}(s)+v_{2}(s)\right) \| \\
\leq & \| \int_{0}^{1}\left[F_{x}\left(t, \sigma v_{1}(t)+(1-\sigma) v_{2}(t)\right)\right. \\
& \left.-F_{x}\left(s, \sigma v_{1}(s)+(1-\sigma) v_{2}(s)\right)\right] d \sigma\left(v_{1}(s)-v_{2}(s)\right) \| \\
& +\| \int_{0}^{1}\left(F_{x}\left(t, \sigma v_{1}(t)+(1-\sigma) v_{2}(t)\right)-A\right) d \sigma \\
& \cdot\left(v_{1}(t)-v_{2}(t)-v_{1}(s)+v_{2}(s)\right) \| \\
\leq & \left(\left\|F_{x t}\right\|_{\infty}(t-s)+\left\|F_{x x}\right\|_{\infty} \rho \varepsilon^{-\alpha}(t-s)^{\alpha}\right)\left\|v_{1}-v_{2}\right\|_{C(D)} \\
& +\left(\left\|F_{x t}\right\|_{\infty}|t-\bar{t}|+\left\|F_{x x}\right\|_{\infty} \rho\right) \varepsilon^{-\alpha}\left[v_{1}-v_{2}\right]_{C_{\alpha}^{\alpha}(D)}(t-s)^{\alpha},
\end{aligned}
$$


so that

$$
\begin{aligned}
& {\left[F\left(\cdot, v_{1}(\cdot)\right)-F\left(\cdot, v_{2}(\cdot)\right)-A\left(v_{1}(\cdot)-v_{2}(\cdot)\right)\right]_{C_{\alpha}^{\alpha}(X)} } \\
\leq & M\left(\left|t_{0}-\bar{t}\right|+\delta+\rho\right)\left\|v_{1}-v_{2}\right\|_{C_{\alpha}^{\alpha}(D)} .
\end{aligned}
$$

Using now (3.3.8), we find

$$
\left\|\Gamma\left(v_{1}\right)-\Gamma\left(v_{2}\right)\right\|_{C_{\alpha}^{\alpha}(D)} \leq 2 \gamma C M\left(\left|t_{0}-\bar{t}\right|+\delta+\rho\right)\left\|v_{1}-v_{2}\right\|_{C_{\alpha}^{\alpha}(D)} .
$$

Therefore, $\Gamma$ is a $1 / 2$-contraction provided

$$
\left\{\begin{array}{l}
\left|t_{0}-\bar{t}\right|+\delta \leq \delta_{0}=(8 \gamma C M)^{-1} \\
\rho<\min \left\{\operatorname{dist}(\bar{u}, \partial \mathcal{O}),(8 \gamma C M)^{-1}\right\}
\end{array}\right.
$$

Let $t_{0}, \delta, \rho$ satisfy (3.3.12). We now show that $\Gamma$ maps $Y$ into itself if $\delta, \rho$ are sufficiently small, $t_{0}$ is sufficiently close to $\bar{t}$, and $x_{0}$ is sufficiently close to $\bar{u}$. If $v \in Y$, we have, denoting by $u_{0}$ the constant function $u_{0}(t)=x_{0}, \forall t \in\left[t_{0}, t_{0}+\delta\right]$,

$$
\begin{aligned}
& \|\Gamma(v)-\bar{u}\|_{C_{\alpha}^{\alpha}(D)} \leq\left\|\Gamma(v)-\Gamma\left(u_{0}\right)\right\|_{C_{\alpha}^{\alpha}(D)}+\left\|\Gamma\left(u_{0}\right)-\bar{u}\right\|_{C_{\alpha}^{\alpha}(D)} \\
\leq & \frac{1}{2}\left\|v-u_{0}\right\|_{C_{\alpha}^{\alpha}(D)}+\left\|\Gamma\left(u_{0}\right)-u_{0}\right\|_{C_{\alpha}^{\alpha}(D)}+\left\|x_{0}-\bar{u}\right\|_{D} \\
\leq & \frac{1}{2}\|v-\bar{u}\|_{C_{\alpha}^{\alpha}(D)}+\left\|\Gamma\left(u_{0}\right)-u_{0}\right\|_{C_{\alpha}^{\alpha}(D)}+\frac{3}{2}\left\|x_{0}-\bar{u}\right\|_{D}
\end{aligned}
$$

since $\Gamma\left(u_{0}\right)-u_{0}$ is the solution $w$ of

$$
w^{\prime}(t)=A w(t)+F\left(t, x_{0}\right), \quad t_{0}<t \leq t_{0}+\delta ; \quad w\left(t_{0}\right)=0
$$

then

$$
\begin{aligned}
\Gamma\left(u_{0}\right)(t)-u_{0}= & \int_{t_{0}}^{t} e^{(t-s) A}\left[F\left(s, x_{0}\right)-F(\bar{t}, \bar{u})\right] d s \\
& +\int_{t_{0}}^{t} e^{(t-s) A} F(\bar{t}, \bar{u}) d s=I_{1}+I_{2}, \quad t_{0} \leq t \leq t_{0}+\delta .
\end{aligned}
$$

By means of Theorem 3.2.17 and (3.3.7) again, we get

$$
\left\|I_{1}\right\|_{C_{\alpha}^{\alpha}(D)} \leq \gamma C\left\|F\left(\cdot, x_{0}\right)-F(\bar{t}, \bar{u})\right\|_{C_{\alpha}^{\alpha}(X)} .
$$

For each $t \in\left[t_{0}, t_{0}+\delta\right]$, we have

$$
\begin{aligned}
\left\|F\left(t, x_{0}\right)-F(\bar{t}, \bar{u})\right\| & \leq\left\|F\left(t, x_{0}\right)-F\left(\bar{t}, x_{0}\right)\right\|+\left\|F\left(\bar{t}, x_{0}\right)-F(\bar{t}, \bar{u})\right\| \\
& \leq\left\|F_{t}\right\|_{\infty}|t-\bar{t}|+\left\|F_{x}\right\|_{\infty}\left\|x_{0}-\bar{u}\right\|_{D} \\
& \leq M\left(\left|t_{0}-\bar{t}\right|+\delta+\left\|x_{0}-\bar{u}\right\|_{D}\right),
\end{aligned}
$$

and for $t_{0}+\varepsilon \leq s<t \leq t_{0}+\delta$,

$$
\varepsilon^{\alpha}\left\|F\left(t, x_{0}\right)-F\left(s, x_{0}\right)\right\| \leq \delta^{\alpha}\left\|F_{t}\right\|_{\infty}|t-s| \leq M \delta|t-s|^{\alpha},
$$

thus

$$
\left\|F\left(\cdot, x_{0}\right)-F(\bar{t}, \bar{u})\right\|_{C_{\alpha}^{\alpha}(X)} \leq M\left(\left|t_{0}-\bar{t}\right|+2 \delta+\left\|x_{0}-\bar{u}\right\|_{D}\right)
$$


This means

$$
\left\|I_{1}\right\|_{C_{\alpha}^{\alpha}(D)} \leq \gamma C M\left(\left|t_{0}-\bar{t}\right|+2 \delta+\left\|x_{0}-\bar{u}\right\|_{D}\right),
$$

so that if

$$
\left|t_{0}-\bar{t}\right|+\delta \leq \delta_{1}=\frac{\rho}{12 \gamma C M},
$$

then

$$
\left\|I_{1}\right\|_{C_{\alpha}^{\alpha}(D)} \leq \frac{\rho}{6}+\gamma C M\left\|x_{0}-\bar{u}\right\|_{D} .
$$

Moreover, since $F(\bar{t}, \bar{u}) \in X=\bar{D}$, then $\lim _{\delta \rightarrow 0}\left\|I_{2}\right\|_{C_{\alpha}^{\alpha}(D)}=0$. Indeed, one checks easily that for every $y \in X$ it holds

$$
\left\|\int_{t_{0}}^{t} e^{(t-s) A} y d s\right\|_{C_{\alpha}^{\alpha}(D)} \leq \text { const. }\|y\|,
$$

with constant independent of $t_{0}, \delta, y$, and that for every $y \in D$,

$$
\lim _{\delta \rightarrow 0}\left\|\int_{t_{0}}^{t} e^{(t-s) A} y d s\right\|_{C_{\alpha}^{\alpha}(D)}=0 .
$$

Therefore (3.3.16) holds for every $y \in \bar{D}=X$. It yields $\lim _{\delta \rightarrow 0}\left\|I_{2}\right\|_{C_{\alpha}^{\alpha}(D)}=0$, so that there is $\delta_{2}>0$ (depending on $\bar{t}$ and $\bar{u}$ ) such that, if $\delta \leq \delta_{2}$, then

$$
\left\|I_{2}\right\|_{C_{\alpha}^{\alpha}(D)} \leq \frac{\rho}{6}
$$

Summing up we find that if

$$
\left|t_{0}-\bar{t}\right|+\delta \leq \min \left\{\delta_{0}, \delta_{1}\right\} \text { and } \delta \leq \delta_{2},
$$

then

$$
\left\|\Gamma\left(u_{0}\right)-u_{0}\right\|_{C_{\alpha}^{\alpha}(D)} \leq \frac{\rho}{3}+\gamma C M\left\|x_{0}-\bar{u}\right\|_{D} .
$$

Further, if $\left\|x_{0}-\bar{u}\right\|_{D} \leq \frac{\rho}{6 \gamma C M+9}$, using (3.3.13), we get

$$
\|\Gamma(v)-\bar{u}\|_{C_{\alpha}^{\alpha}(D)} \leq \rho .
$$

This means $\Gamma$ maps $Y$ into itself. Therefore there is a unique fixed point $u$ of $\Gamma$ in $Y$, which is a solution of (3.3.3). Statement (i) follows.

Let us prove that statement (ii) holds. The derivatives of the functions in the range of $\Gamma$ belong to $B_{\alpha}\left(\left(t_{0}, t_{0}+\delta\right] ;(X, D)_{\alpha, \infty}\right)$ thanks to Corollary 3.2.18(ii). Since $u$ is a fixed point of $\Gamma$, then $u^{\prime}$ is in $B_{\alpha}\left(\left(t_{0}, t_{0}+\delta\right] ;(X, D)_{\alpha, \infty}\right)$.

Concerning (3.3.4), set

$$
Y^{\prime}=\left\{v \in Y ; \lim _{\varepsilon \rightarrow 0}[v]_{C_{\alpha}^{\alpha}\left(\left(t_{0}, t_{0}+\varepsilon\right] ; D\right)}=0\right\} .
$$


$Y^{\prime}$ is closed in $C_{\alpha}^{\alpha}\left(\left(t_{0}, t_{0}+\delta\right] ; D\right)$, and $\Gamma\left(Y^{\prime}\right) \subset Y^{\prime}$ (the proof is the same used above to show that $\Gamma(Y) \subset Y$ ). The fixed point $u$ of $\Gamma$ belongs to $Y^{\prime}$, which means that $u$ satisfies (3.3.4).

Let us prove uniqueness. If $u, v$ are solutions of (3.3.3) belonging to $C\left(\left[t_{0}, t_{0}+\right.\right.$ $\delta] ; D) \cap C_{\beta}^{\beta}\left(\left(t_{0}, t_{0}+\delta\right] ; D\right)$ for some $\beta \in(0,1)$, set

$$
t_{1}=\sup \left\{t \in\left[t_{0}, t_{0}+\delta\right] ;\left.u\right|_{\left[t_{0}, t\right]}=\left.v\right|_{\left[t_{0}, t\right]}\right\} .
$$

Assume by contradiction that $t_{1}<t_{0}+\delta$. Then $u\left(t_{1}\right)=v_{1}$, because both $u$ and $v$ are continuous. Setting $x_{1}=u\left(t_{1}\right)=v\left(t_{1}\right)$, both $u$ and $v$ are solution of

$$
w^{\prime}(t)=F(t, w(t)), \quad t_{1}<t \leq t_{0}+\delta ; \quad w\left(t_{1}\right)=x_{1},
$$

where $x_{1} \in \mathcal{O}$ and $F\left(t_{1}, x_{1}\right)=u^{\prime}\left(t_{1}\right)$. Taking $\bar{t}=t_{1}, \bar{u}=x_{1}$, and replacing $\alpha$ by any $\gamma<\beta$ in point (i), we obtain the existence of $\delta_{1}>0$ such that problem (3.3.18) has a unique strict solution in the set

$$
\begin{aligned}
Y_{1}= & \left\{u \in C_{\gamma}^{\gamma}\left(\left(t_{1}, t_{1}+\delta_{1}\right] ; D\right) \cap C\left(\left[t_{1}, t_{1}+\delta_{1}\right] ; D\right) ;\right. \\
& \left.u\left(t_{1}\right)=x_{1},\left\|u(\cdot)-x_{1}\right\|_{C_{\gamma}^{\gamma}\left(\left(t_{1}, t_{1}+\delta_{1}\right] ; D\right)} \leq \rho_{1}\right\},
\end{aligned}
$$

provided $\rho_{1}$ and $\delta_{1}$ are sufficiently small. Now, both $\left.u\right|_{\left[t_{1}, t_{1}+\delta_{1}\right]}$ and $\left.v\right|_{\left[t_{1}, t_{1}+\delta_{1}\right]}$ belong to $Y_{1}$ if $\delta_{1}$ is small: in particular, $\left\|u(t)-x_{1}\right\|_{D} \leq \rho_{1},\left\|v(t)-x_{1}\right\|_{D} \leq \rho_{1}$ for $\delta_{1}$ small, because $u$ and $v$ are continuous and they assume the value $x_{1}$ at $t=t_{1}$; moreover for $t_{0}<t_{1}+\varepsilon \leq s<t \leq t_{1}+\delta_{1} \leq t_{0}+\delta$ and for every $a \in(0,1)$ it holds

$$
\left.\leq \int_{t_{1} \leq s<t \leq t_{1}+\delta_{1}}\|u(t)-u(s)\|_{D}\right)^{a}\left([u]_{C_{\beta}^{\beta}\left(\left(t_{0}, t_{0}+\delta\right] ; D\right)}(t-s)^{\beta} \varepsilon^{-\beta}\right)^{1-a} .
$$

Choosing $a=1-\gamma / \beta$, we get

$$
[u]_{C_{\gamma}^{\gamma}\left(\left(t_{1}, t_{1}+\delta_{1}\right] ; D\right)} \leq\left(\sup _{t_{1} \leq s<t \leq t_{1}+\delta_{1}}\|u(t)-u(s)\|_{D}\right)^{a}\left([u]_{C_{\beta}^{\beta}\left(\left(t_{0}, t_{0}+\delta\right] ; D\right)}\right)^{1-a},
$$

and, similarly,

$$
[v]_{C_{\gamma}^{\gamma}\left(\left(t_{1}, t_{1}+\delta_{1}\right] ; D\right)} \leq\left(\sup _{t_{1} \leq s<t \leq t_{1}+\delta_{1}}\|v(t)-v(s)\|_{D}\right)^{a}\left([v]_{C_{\beta}^{\beta}\left(\left(t_{0}, t_{0}+\delta\right] ; D\right)}\right)^{1-a} .
$$

Since both $u$ and $v$ are continuous with values in $D$, we get

$$
[u]_{C_{\gamma}^{\gamma}\left(\left(t_{1}, t_{1}+\delta_{1}\right] ; D\right)} \leq \rho_{1}, \quad[v]_{C_{\gamma}^{\gamma}\left(\left(t_{1}, t_{1}+\delta_{1}\right] ; D\right)} \leq \rho_{1},
$$

provided $\delta_{1}$ is small enough.

Therefore, $\left.u\right|_{\left[t_{1}, t_{1}+\delta_{1}\right]}=\left.v\right|_{\left[t_{1}, t_{1}+\delta_{1}\right]}$, but this contradicts the definition of $t_{1}$. Hence $u=v$, and statement (ii) follows. 
Corollary 3.3.2. Under the assumptions of Theorem 3.3.1, for every $\bar{t} \in[0, T]$ and $\bar{u} \in \mathcal{O}$ there is $K=K(\bar{t}, \bar{u})>0$ such that for every to $t_{0} \in[\bar{t}-r, \bar{t}+r]$, and $x_{0}, x_{1} \in \mathcal{O}$ with $\left\|x_{i}-\bar{u}\right\|_{D} \leq r$, we have, denoting by $u_{i}$ the solution of (3.3.3) with initial value $x_{i}, i=0,1$,

$$
\begin{aligned}
& \left\|u_{0}-u_{1}\right\|_{C_{\alpha}^{\alpha}\left(\left(t_{0}, t_{0}+\delta\right] ; D\right)}+\left\|u_{0}^{\prime}-u_{1}^{\prime}\right\|_{C_{\alpha}^{\alpha}\left(\left(t_{0}, t_{0}+\delta\right] ; X\right)} \\
& +\sup _{t_{0} \leq t \leq t_{0}+\delta}\left(t-t_{0}\right)^{\alpha}\left\|u_{0}^{\prime}(t)-u_{1}^{\prime}(t)\right\|_{(X, D)_{\alpha, \infty}} \leq K\left\|x_{0}-x_{1}\right\|_{D} .
\end{aligned}
$$

If the initial datum $x_{0}$ is "more regular", in the sense that

$$
F\left(t_{0}, x_{0}\right) \in(X, D)_{\alpha, \infty}
$$

then the local solution of (3.3.3) is uniformly $\alpha$-Hölder continuous with values in $D$ up to $t=t_{0}$, and the corresponding continuous dependence result holds, as the following theorem shows.

Theorem 3.3.3. Let the assumptions of Theorem 3.3.1 be satisfied, and let in addition (3.3.19) hold. If $u:\left[t_{0}, t_{0}+\delta\right] \rightarrow D$ is the solution of (3.3.3) given by Theorem 3.3.1, then

$$
u \in B U C^{\alpha}\left(\left[t_{0}, t_{0}+\delta\right] ; D\right) \cap B U C^{1+\alpha}\left(\left[t_{0}, t_{0}+\delta\right] ; X\right), u^{\prime} \in B\left(\left[t_{0}, t_{0}+\delta\right] ;(X, D)_{\alpha, \infty}\right) .
$$

Moreover, for every $\bar{t} \in[0, T], \bar{u} \in \mathcal{O}$ such that $F(\bar{t}, \bar{u}) \in(X, D)_{\alpha, \infty}$, there are $r_{0}=$ $r_{0}(\bar{t}, \bar{u})>0, K_{0}=K_{0}(\bar{t}, \bar{u})>0$ with the following property: for every $t_{0} \in\left[\bar{t}-r_{0}, \bar{t}+r_{0}\right]$, and $x_{0}, x_{1} \in \mathcal{O}$ such that

$$
\left\|x_{i}-\bar{u}\right\|_{D} \leq r_{0}, \quad\left\|F\left(t_{0}, x_{i}\right)-F(\bar{t}, \bar{u})\right\|_{(X, D)_{\alpha, \infty}} \leq r_{0}, i=0,1,
$$

we have, denoting by $u_{i}, i=0,1$, the solution of (3.3.3) with initial value $x_{i}$,

$$
\begin{gathered}
\left\|u_{0}-u_{1}\right\|_{B U C^{\alpha}\left(\left[t_{0}, t_{0}+\delta\right] ; D\right)}+\left\|u_{0}^{\prime}-u_{1}^{\prime}\right\|_{B U C^{\alpha}\left(\left[t_{0}, t_{0}+\delta\right] ; X\right)}+\left\|u_{0}^{\prime}-u_{1}^{\prime}\right\|_{B\left(\left[t_{0}, t_{0}+\delta\right] ;(X, D)_{\alpha, \infty}\right)} \\
\leq K_{0}\left(\left\|x_{0}-x_{1}\right\|_{D}+\left\|F\left(t_{0}, x_{0}\right)-F\left(t_{0}, x_{1}\right)\right\|_{(X, D)_{\alpha, \infty}}\right) .
\end{gathered}
$$

\subsubsection{The maximally defined solution}

Throughout the subsection, $F:[0, T] \times \mathcal{O} \rightarrow X$ is a nonlinear function satisfying assumption (3.3.2). For each $u_{0} \in \mathcal{O}$, Theorem 3.3 .1 yields existence and uniqueness of a local solution $u$ of (3.3.1) in the space $C_{\alpha}^{\alpha}((0, \delta] ; D)$, enjoying property (3.3.4). Since $u \in B U C^{\alpha}([\delta / 2, \delta] ; D) \cap B U C^{1+\alpha}([\delta / 2, \delta] ; X)$, then $u^{\prime}(\delta)=F(\delta, u(\delta))$ belongs to $(X, D)_{\alpha, \infty}$ (see Proposition 3.1.29), so that, thanks to Theorem 3.3.3, $u$ can be continued to some interval $\left[\delta, \delta_{1}\right]$ with $\delta_{1}>\delta$, in such a way the extension belongs to $C_{\alpha}^{\alpha}\left(\left(0, \delta_{1}\right] ; D\right)$. So we define $u=u\left(\cdot ; u_{0}\right)$ by

$$
\left\{\begin{array}{c}
I=I\left(u_{0}\right)=\bigcup\left\{[0, \delta] ; \text { problem }(3.3 .1) \text { has a solution } u_{\delta} \in\right. \\
\left.C([0, \delta] ; D) \cap C_{\beta}^{\beta}((0, \delta] ; D) \text { for some } \beta \in(0,1)\right\}, \\
u: I \rightarrow D, \quad u(t)=u_{\delta}(t) \text { for } t \in[0, \delta] \subset I .
\end{array}\right.
$$


$u$ is well defined thanks to the uniqueness part of Theorem 3.3.1. We shall see in the next proposition that $u$ has no continuous extension with values in $D$. So, it is the maximally defined solution of problem (3.3.1). We set

$$
\tau=\tau\left(u_{0}\right)=\sup I\left(u_{0}\right) .
$$

Proposition 3.3.4. If $u\left(\cdot ; u_{0}\right)$ is uniformly continuous with values in $D$, then either

$$
\lim _{t \rightarrow \tau\left(u_{0}\right)} u\left(t ; u_{0}\right) \in \partial \mathcal{O},
$$

or

$$
I\left(u_{0}\right)=[0, T] .
$$

A sufficient condition for $u$ be uniformly continuous in $I$ is given by next lemma.

Lemma 3.3.5. Let the assumptions of Theorem 3.3.1 hold, and let $u: I \rightarrow D$ be the maximally defined solution of problem (3.3.1). If the orbit

$$
\{u(t) ; 0 \leq t<\tau\}
$$

is relatively compact in $D$, then $u:[0, \tau) \rightarrow D$ is uniformly continuous.

The maximally defined solution depends continuously on the initial value, as the following proposition show.

Proposition 3.3.6. Let $\bar{u} \in \mathcal{O}$, and fix $\bar{\tau} \in(0, \tau(\bar{u}))$. Then there are $\varepsilon=\varepsilon(\bar{u}, \bar{\tau})>0$, $H=H(\bar{u}, \bar{\tau})>0$ such that if

$$
u_{0} \in \mathcal{O} \quad \text { and } \quad\left\|u_{0}-\bar{u}\right\|_{D} \leq \varepsilon,
$$

then

$$
\tau\left(u_{0}\right) \geq \bar{\tau}
$$

and

$$
\begin{aligned}
& \left\|u\left(\cdot ; u_{0}\right)-u(\cdot ; \bar{u})\right\|_{C_{\alpha}^{\alpha}((0, \bar{\tau}] ; D)}+\left\|u_{t}\left(\cdot ; u_{0}\right)-u_{t}(\cdot ; \bar{u})\right\|_{C_{\alpha}^{\alpha}((0, \bar{\tau}] ; X)} \\
& \quad+\sup _{0<t \leq \bar{\tau}} t^{\alpha}\left\|u_{t}\left(\cdot ; u_{0}\right)-u_{t}(\cdot ; \bar{u})\right\|_{(X, D)_{\alpha, \infty}} \leq H\left\|u_{0}-\bar{u}\right\|_{D} .
\end{aligned}
$$




\section{Chapter 4}

\section{Stabilization of flows through porous media}

In this chapter we study the motion of an incompressible homogeneous Newtonian fluid in a rigid porous medium of infinite extent. The fluid is bounded below by a fixed layer having an external source, and above by a free surface moving under the influence of gravity. The flow is governed by Darcy's law. In [12], J. Escher and G. Simonett studied the case of the fluid bounded below by a fixed impermeable layer, instead of a fixed layer having an external source, i.e. $b \equiv 0$. They proved that the problem $(P)$ with $b \equiv 0$ is locally in time well-posed. However, the question of the stability of the equilibrium solution $(u, f) \equiv(c, c)$ was not addressed in [12]. In this chapter, we shall prove that the equilibrium solution $(u, f) \equiv(c, c)$ is exponentially stable if the term injection rate $b$ satisfies $b(c)=0$ and $b^{\prime}(c)>0$.

\subsection{Modelling of flows through porous media}

In the following, we describe the physical model we are interested in. We consider a system composed of two fluid phases that simultaneously occupy the entire void space of a porous medium. We shall refer to one fluid (subscript $w$ ) as the wetting fluid (e.g. water), and to the other one (subscript $n$ ) as the nonwetting fluid (e.g. air). In addition, subscript $s$ denotes the solid (e.g. rock). Here the wetting fluid is an incompressible homogenous Newtonian fluid.

\subsubsection{The mass balance equations}

Our starting point is the mass balance equation for the wetting fluid in a rigid, isotropic and linearly elastic porous medium. The mass balance for the fluid phase is expressed 
by

$$
\begin{aligned}
\frac{\partial\left(n S_{w} \rho_{w}\right)}{\partial t}= & -\nabla \cdot\left\{n S_{w}\left(\rho_{w} \mathbf{V}_{w}-\mathbf{D}_{w} \cdot \nabla \rho_{w}\right)\right\} \\
& -\overline{\rho_{w}\left(\mathbf{V}_{w}-\mathbf{V}_{w n}\right) \cdot \nu_{w n}}{ }^{w n} \Sigma_{w n} \\
& -{\overline{\rho_{w}\left(\mathbf{V}_{w}-\mathbf{V}_{w s}\right) \cdot \nu_{w s}}}^{w s} \Sigma_{w s},
\end{aligned}
$$

(see (2.4.10) in [5]), where $n$ denotes the porosity, $\rho_{w}, S_{w}$ and $\mathbf{V}_{w}$ denote the density, the saturation and the mass weighted velocity of the wetting fluid phase, respectively. The term $\rho_{w} \mathbf{V}_{w}$ expresses the wetting fluid's mass flux, while $-\mathbf{D}_{w} \cdot \nabla \rho_{w}$ expresses the wetting fluid's dispersive mass flux. The symbols $\mathbf{V}_{w n}$ and $\mathbf{V}_{w s}$ denote the velocity of (wetting) fluid-(nonwetting) fluid interface $S_{w n}$ and (wetting) fluid-solid interface $S_{w s}$, respectively. Moreover, the term $-\overline{\rho_{w}\left(\mathbf{V}_{w}-\mathbf{V}_{w n}\right) \cdot \nu_{w n}}{ }^{w n} \Sigma_{w n}$ expresses the influx of the mass across the fluid-fluid interface relative to the possibly moving fluid-fluid interface, and the term $-{\overline{\rho_{w}\left(\mathbf{V}_{w}-\mathbf{V}_{w s}\right) \cdot \nu_{w s}}}^{w s} \Sigma_{w s}$ expresses the influx of the mass across the fluid-solid interface relative to the possibly moving fluid-solid interface. Following J. Bear and Y. Bachmat[5], we assume that

$A_{1}$ The mass fluxes due to dispersion is much smaller than the mass flux and will therefore be neglected. i.e.

$$
\left|\rho_{w} \mathbf{V}_{w}\right| \gg\left|\mathbf{D}_{w} \cdot \nabla \rho_{w}\right|
$$

$A_{2}$ The fluid-solid interface is a material surface with respect to the wetting fluid's mass, i.e., no mass of the wetting fluid phase crosses $S_{w s}$.

$A_{3}$ The fluid-fluid interface is also a material surface with respect to the wetting fluid's mass, i.e., no mass of the wetting fluid phase crosses $S_{w n}$.

With these three assumptions, (4.1.1) reduces to

$$
\frac{\partial\left(n S_{w} \rho_{w}\right)}{\partial t}=-\nabla \cdot\left(n S_{w} \rho_{w} \mathbf{V}_{w}\right)
$$

Equation (4.1.2) is commonly used as the basic (macroscopic) differential mass balance equation of a phase. So the mass balance for the solid phase can be expressed by an equation similar to (4.1.2), namely

$$
\frac{\partial\left\{(1-n) \rho_{s}\right\}}{\partial t}=-\nabla \cdot\left\{(1-n) \rho_{s} \mathbf{V}_{s}\right\}
$$

where $\rho_{s}$ and $\mathbf{V}_{s}$ are the solid's density and velocity, respectively. Let

$$
\mathbf{q}_{w}=n S_{w} \mathbf{V}_{w} \quad \text { and } \quad \mathbf{q}_{w r}=n S_{w}\left(\mathbf{V}_{w}-\mathbf{V}_{s}\right)
$$

be the mass weighted specific discharge and the relative mass weighted specific discharge (i.e., relative to the solid), respectively. 
The fluid's mass balance (4.1.2) can be rewritten in the form

$$
\nabla \cdot\left(\rho_{w} \mathbf{q}_{w r}\right)+n S_{w} \frac{D_{s} \rho_{w}}{D t}+S_{w} \rho_{w} \frac{D_{s} n}{D t}+n \rho_{w} \frac{D_{s} S_{w}}{D t}+n S_{w} \rho_{w} \nabla \cdot \mathbf{V}_{s}=0,
$$

where $D_{s}(\cdot) / D t=\partial(\cdot) / \partial t+\mathbf{V}_{s} \cdot \nabla(\cdot)$ is the material derivative with respect to the solid.

Equation (4.1.3) can be rewritten in the form

$$
\frac{1}{1-n} \frac{D_{s}(1-n)}{D t}+\frac{1}{\rho_{s}} \frac{D_{s} \rho_{s}}{D t}+\nabla \cdot \mathbf{V}_{s}=0
$$

By eliminating $\nabla \cdot \mathbf{V}_{s}$ from (4.1.4) and (4.1.5), we obtain

$$
\nabla \cdot\left(\rho_{w} \mathbf{q}_{w r}\right)+n S_{w} \frac{D_{s} \rho_{w}}{D t}+\frac{S_{w} \rho_{w}}{1-n} \frac{D_{s} n}{D t}+n \rho_{w} \frac{D_{s} S_{w}}{D t}-\frac{n S_{w} \rho_{w}}{\rho_{s}} \frac{D_{s} \rho_{s}}{D t}=0 .
$$

$A_{4}$ The solid phase (at the microscopic level!) preserves its volume. This means

$$
\frac{D_{s} \rho_{s}}{D t}=0
$$

With assumption $A_{4}$, equation (4.1.6) reduces to

$$
\nabla \cdot\left(\rho_{w} \mathbf{q}_{w r}\right)+n S_{w} \frac{D_{s} \rho_{w}}{D t}+\frac{S_{w} \rho_{w}}{1-n} \frac{D_{s} n}{D t}+n \rho_{w} \frac{D_{s} S_{w}}{D t}=0 .
$$

$A_{5}$ The solid matrix is macroscopically fixed in space, i.e. $\mathbf{V}_{s}=0$.

With assumption $A_{4}$ and $A_{5}$, equation (4.1.5) reduces to

$$
\frac{\partial n}{\partial t}=0
$$

With assumption $A_{5}$, we know that

$$
\mathbf{q}_{w}=\mathbf{q}_{w r}
$$

Moreover, equation (4.1.7) also reduces to

$$
\nabla \cdot\left(\rho_{w} \mathbf{q}_{w}\right)+n \frac{\partial\left(S_{w} \rho_{w}\right)}{\partial t}=0
$$

$A_{6}$ The density is constant, i.e., $\rho_{w}=$ const., and the microscopic boundary of the wetting phase is such that $\frac{\partial\left(n S_{w}\right)}{\partial t}=0$.

With assumption $A_{6}$ and (4.1.8), equation (4.1.10) reduces to

$$
\nabla \cdot \mathbf{q}_{w}=0
$$

This conservation law will be of fundamental importance in the following. 


\subsubsection{The momentum balance equation}

We next assume that

$A_{7}$ The wetting fluid is microscopically isochoric flow.

$A_{8}$ The fluid-solid interface is much larger than the fluid-fluid interface, i.e., $S_{w s} \gg$ $S_{w n}$.

With assumption $A_{8}$, we know

$$
\int_{S_{w s}} \sigma_{w} \cdot \nu_{w} d S \gg \int_{S_{w n}} \sigma_{w} \cdot \nu_{w} d S
$$

where $\sigma_{w}$ denotes the stress tensor of the wetting fluid. This means the fluid-fluid momentum transfer is much smaller than the fluid-solid one. Therefore, we may neglect the momentum transferred between the fluid phases that together occupy the void space.

With assumption $A_{5}, A_{7}$ and $A_{8}$, the momentum balance equation of the wetting fluid can be written in the form

$$
\begin{aligned}
\rho_{w}\left\{\frac{\partial q_{w i}}{\partial t}+\frac{\partial}{\partial x_{j}}\left(\frac{q_{w i} q_{w j}}{\theta_{w}}\right)\right\}= & -\theta_{w}\left(\frac{\partial p_{w}}{\partial x_{j}}+\rho_{w} g \frac{\partial z}{\partial x_{j}}\right) T_{w j i}^{*} \\
& +\mu_{w} \frac{\partial^{2} q_{w i}}{\partial x_{j} \partial x_{j}}-\mu_{w} \frac{C_{w}}{\Delta_{w}^{2}} \alpha_{i j}^{(w s)} q_{w j}
\end{aligned}
$$

(see (2.6.48) in [5]), where we use Einstein's summation convention. In (4.1.12), $\theta_{w}(=$ $\left.n S_{w}\right)$ and $p_{w}$ denote the content and the pressure of the wetting fluid, respectively. Moreover, $g$ is gravity acceleration, $z$ is the $z$-coordinate of the position, $\mu_{w}$ is the wetting fluid's dynamic viscosity, $C_{w}$ is a shape factor associated with the ws-surface area, on the $w$ side of this surface. Moreover, $\Delta_{w}=\theta_{w} / \Sigma_{w s}$, here $\Sigma_{w s}$ is the specific area of the fluid-solid interface within per unit volume of the consider domain, $\mathbf{T}^{*}{ }_{w}$ and $\alpha^{(w s)}$ are two tensorial properties of the configuration of the $w s$-surface in saturated, single phase flow.

In (4.1.12), the term on the l.h.s. represents the inertial force acting on the wetting fluid, per unit volume of porous medium.

The first term on the r.h.s. of (4.1.12) represents the resultant force acting on the wetting fluid, due to gravity and to pressure gradient, per unit volume of porous medium.

The second term on the r.h.s. of (4.1.12) represents the force acting on the wetting fluid, due to the viscous resistance to its flow inside the wetting fluid, per unit volume of porous medium.

The last term on the r.h.s. of (4.1.12) expresses the viscous resistance, or viscous drag force exerted by the solid phase on the wetting fluid at their contact surfaces, per unit volume of porous medium. 


\subsubsection{Darcy's law}

We now introduce the fundamental construction relation for the velocity of the wetting fluid.

$A_{9}$ The wetting fluid is governed by the Darcy's law (cf. [20]), i.e.,

$$
\mathbf{q}_{w}=-\mathbf{K} \nabla u
$$

Namely, the flow in a given domain is such that the viscous resistance force, due to the momentum transfer at the solid-fluid interface, is much larger than both the inertial force and the viscous resistance to the flow inside the fluid, i.e.

$$
\left|\mu_{w} \frac{C_{w}}{\Delta_{w}^{2}} \alpha_{i j}^{(w s)} q_{w j}\right| \gg\left|\rho_{w}\left\{\frac{\partial q_{w i}}{\partial t}+\frac{\partial}{\partial x_{j}}\left(\frac{q_{w i} q_{w j}}{\theta_{w}}\right)\right\}\right|,
$$

and

$$
\left|\mu_{w} \frac{C_{w}}{\Delta_{w}^{2}} \alpha_{i j}^{(w s)} q_{w j}\right| \gg\left|\mu_{w} \frac{\partial^{2} q_{w i}}{\partial x_{j} \partial x_{j}}\right| .
$$

Under these conditions, the momentum balance equation (4.1.12) reduces to

$$
q_{w j}=-\frac{k_{w j l}}{\mu_{w}}\left\{\frac{\partial p_{w}}{\partial x_{l}}+\rho_{w} g \frac{\partial z}{\partial x_{l}}\right\}
$$

where

$$
k_{w j l}=\frac{\theta_{w} \Delta_{w}^{2}}{C_{w}}\left(\alpha_{j i}^{(w s)}\right)^{-1} T_{w i l}^{*}
$$

is called the effective permeability for the wetting fluid in a porous medium. Assumption $A_{6}$ permits to introduce a so-called piezometric head $u$ (see [5] p.175), by setting

$$
u(x, y):=\frac{p_{w}(x, z)}{\rho_{w} g}+z, \quad(x, z) \in \mathbb{R}^{2} \times \mathbb{R} .
$$

Then, (4.1.13) reduces to

$$
q_{w j}=-K_{j i} \frac{\partial u}{\partial x_{i}}
$$

where the second rank symmetrical tensor

$$
K_{j i}=k_{w j i} \frac{\rho_{w} g}{\mu_{w}}
$$

is a coefficient called hydraulic conductivity. Note that assumption $A_{9}$ is the same as equation (4.1.16). In general $\mathbf{K}$ is a symmetric, uniformly positive definite $3 \times 3$-matrix. However, for homogeneous and isotropic media, we have $\mathbf{K}=k \cdot i d_{\mathbb{R}^{3}}$ for some positive constant k, (see [5] p.189).

Therefore, by means of Darcy's law, the mass balance equation (4.1.11) reduces to

$$
\Delta u=0 .
$$


$A_{10}$ The content of the wetting fluid is independent of position, i.e., $\nabla \theta_{w}=0$.

With assumption $A_{6}$ and $A_{10}$, we know that $\theta_{w}=$ const. . Moreover, the Darcy's law can be rewritten in the form

$$
\mathbf{V}_{w}=-\frac{k}{n S_{w}} \nabla u=-\frac{k}{\theta_{w}} \nabla u
$$

\subsubsection{The boundary conditions}

To describe the shape of the moving boundary, we assume that

$A_{11}$ The capillary pressure $p_{c}=p_{n}-p_{w}$ is continuous, here $p_{n}$ denotes the pressure of the nonwetting fluid.

We assume that the air in the unsaturated zone is at atmospheric pressure, normalized to be zero i.e. $p_{n}=0$. With assumption $A_{11}$, we know that $p_{w}$ is continuous and the shape of the moving fluid-fluid interface $\Gamma_{t}$ at time $t$ is then given by the relation

$$
(x, z) \in \Gamma_{t} \text { if and only if } z=\sup \left\{y \in \mathbb{R} ; p_{w}(t, x, y)>0\right\} .
$$

Finally, we assume that the interface $\Gamma_{t}$ is parameterized over $\mathbb{R}^{2}$, i.e., we assume that there is a function $f$ such that

$$
(x, z) \in \Gamma_{t} \text { if and only if } z=f(t, x) .
$$

In particular, we find that $\Gamma_{t}=\Gamma_{f(t)}=\operatorname{graph}(f(t, \cdot))$. Moreover, (4.1.15) and (4.1.19) fuse to

$$
u=f \text { on } \Gamma_{f} .
$$

Finally, setting $F(t, y):=z-f(t, x)$ for $y=(x, z) \in \mathbb{R}^{2} \times \mathbb{R}$ and $t \geq 0$, the interface $\Gamma_{f}$ can also be described by the conservative property that $F$ is identically equal to zero on $\Gamma_{f}$. Hence, we get

$$
\frac{d}{d t} F(t, y)=\partial_{t} F(t, y)+\nabla F(t, y) \cdot \dot{y}=0 \text { on } \Gamma_{f} .
$$

Using (4.1.18), we may express the velocity $\dot{y}$ by $-\frac{k}{\theta_{w}} \nabla u$. Consequently, observing $\partial_{t} F=-\partial_{t} f, \nabla F=\left(-\nabla_{x} f, 1\right)$, and $\nu$ is the outer normal of $\Gamma_{t}$,

$$
\nu:=\frac{\left(-\nabla_{x} f, 1\right)}{\sqrt{1+\left|\nabla_{x} f\right|^{2}}} .
$$

(4.1.21) becomes

$$
\partial_{t} f+\frac{k}{\theta_{w}} \sqrt{1+\left|\nabla_{x} f\right|^{2}} \partial_{\nu} u=0 \text { on } \Gamma_{f} .
$$


$A_{12}$ The porous medium is bounded below by a fixed layer $\Gamma_{0}$ with an external source $\Gamma^{m}$, and this external source depends on the wn-interface.

With assumption $A_{1}$ and $A_{12}$, the general boundary condition for mass on $\Gamma_{0}$ can be expressed by

$$
\left(\rho_{w} \mathbf{V}_{w}+\Gamma^{m}\right) \cdot \nu=0
$$

(see (2.7.6) in [5]), where $\nu=(0,-1)$. Using (4.1.18), equation (4.1.23) can be rewritten in the form

$$
\partial_{z} u=\frac{\theta_{w}}{k \rho_{w}} b
$$

where $b=-\Gamma^{m} \cdot \nu$ is called interface-dependent injection rate. Since $\boldsymbol{\Gamma}^{m}$ depends on the $w n$-interface, the function $b$ also depends on $f$.

Finally, adding initial condition for the interface $f$, i.e., $f(0, \cdot)=f_{0}$, as well as the normalization $\lim _{|(x, z)| \rightarrow \infty} u(t, x, z)=c$, we see that (4.1.17), (4.1.20), (4.1.22), and (4.1.24) fit, up to multiplicative constants, into

$$
\begin{aligned}
& \Delta u=0 \quad \text { in } \quad \Omega_{f, T}, \\
& \partial_{z} u=b(f(t, x)) \text { on } \Gamma_{0, T} \text {, } \\
& u=f \quad \text { on } \Gamma_{f, T}, \\
& \lim _{|(x, z)| \rightarrow \infty} u(t, x, z)=c \text { on }[0, T) \text {, } \\
& \partial_{t} f+\sqrt{1+\left|\nabla_{x} f\right|^{2}} \partial_{\nu} u=0 \text { on } \dot{\Gamma}_{f, T} \text {, } \\
& f(0, \cdot)=f_{0} \text { on } \mathbb{R}^{2} \text {, }
\end{aligned}
$$

where $T>0$ is fixed, and

$$
\begin{aligned}
& \Omega_{f(t)}:=\left\{(x, z) \in \mathbb{R}^{2} \times \mathbb{R} ; 0<z<f(t, x)\right\}, \\
& \Gamma_{f(t)}:=\left\{(x, z) \in \mathbb{R}^{2} \times \mathbb{R} ; z=f(t, x)\right\}, \\
& \Omega_{f, T}:=\left\{(t, y) \in[0, T) \times \mathbb{R}^{3} ; y \in \Omega_{f(t)}\right\}, \\
& \Gamma_{f, T}:=\left\{(t, y) \in[0, T) \times \mathbb{R}^{3} ; y \in \Gamma_{f(t)}\right\}, \\
& \dot{\Gamma}_{f, T}:=\left\{(t, y) \in(0, T) \times \mathbb{R}^{3} ; y \in \Gamma_{f(t)}\right\}, \\
& \Gamma_{0, T}:=[0, T) \times \Gamma_{0} .
\end{aligned}
$$

\subsection{The full system}

In this section, we shall describe the problem we investigate in detail. Now we consider the case of the problem $(P)_{f_{0}, c}$ in one space dimension (see Figure 1), 


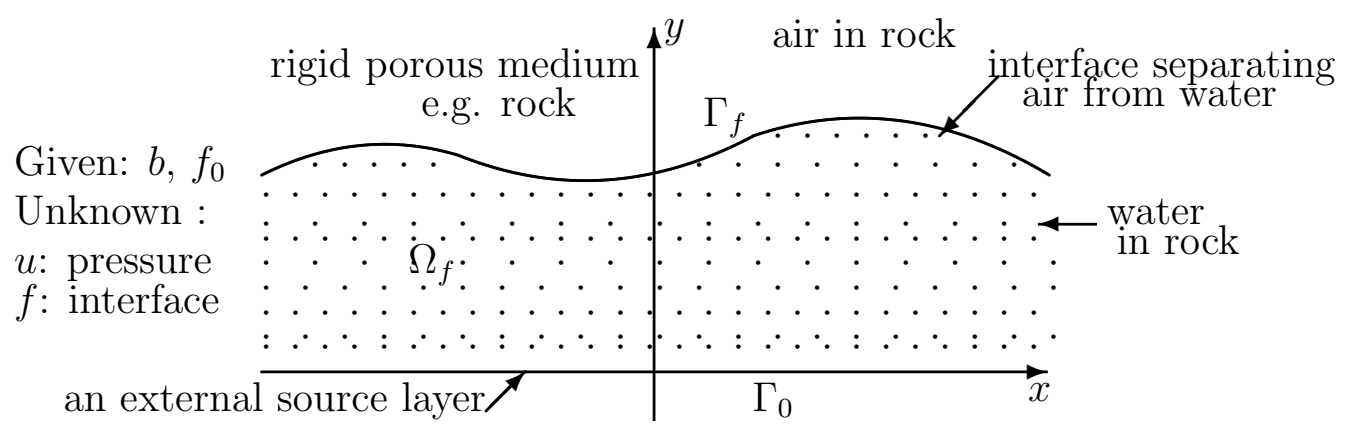

Figure 1

i.e.

$$
\begin{aligned}
\Delta u(t, x, y) & =0, & & t \in J,(x, y) \in \Omega_{f(t)}, \\
\left.\frac{\partial u}{\partial y}(t, x, y)\right|_{y=0} & =b(f(t, x)), & & t \in J, x \in \mathbb{R}, \\
\left.u(t, x, y)\right|_{y=f(t, x)} & =f(t, x), & & t \in J, x \in \mathbb{R}, \\
\lim _{|x|+|y| \rightarrow \infty} u(t, x, y) & =c, & & t \in J,(x, y) \in \Omega_{f(t)}, \\
\partial_{t} f(t, x)+\left(-f_{x}, 1\right) \cdot \nabla u(t, x, y) & =0, & & t \in \dot{J},(x, y) \in \Gamma_{f(t)}, \\
f(0, x) & =f_{0}(x), & & x \in \mathbb{R},
\end{aligned}
$$

where $T>0$ is fixed, and $J=[0, T), \dot{J}=J \backslash\{0\}$. The injection rate $b$ is a given smooth function from $\mathbb{R}$ to $\mathbb{R}$.

Recall that $\mathfrak{A}=\left\{g \in B C^{2}(\mathbb{R}) ; \inf _{x \in \mathbb{R}}\{c+g(x)\}>0\right\}$. Given $s>0$, let $h^{s}(\mathbb{R})$ be the closure of $\mathcal{S}(\mathbb{R})$ in $B U C^{s}(\mathbb{R})$, and

$$
h^{s}:=h^{s}(\mathbb{R}), \quad \text { and } \quad h_{\mathfrak{A}}^{s}:=h^{s} \cap \mathfrak{A} .
$$

Remark 4.2.1. For $s \geq 2, h_{\mathfrak{A}}^{s}$ is open in $h^{s}$. Indeed, for $\forall g \in h_{\mathfrak{A}}^{s}$, let

$$
M=\inf _{x \in \mathbb{R}}\{c+g(x)\},
$$

then $M>0$. Thanks to (2.1.1) and the definition of $h^{s}$, we have $h^{s} \hookrightarrow B U C^{2}(\mathbb{R}) \hookrightarrow$ $B C(\mathbb{R})$. So that there exists $C>0$ such that

$$
\|u\|_{\infty} \leq C\|u\|_{h^{s}}, \quad \forall u \in h^{s} .
$$

For $\forall h \in h^{s}$ satisfying $\|h-g\|_{h^{s}} \leq M / 2 C$, we get

$$
\|h-g\|_{\infty} \leq C\|h-g\|_{h^{s}} \leq C \frac{M}{2 C}=\frac{M}{2} .
$$

Then we have

$$
c+h(x)=c+g(x)+h(x)-g(x) \geq M-\frac{M}{2}=\frac{M}{2}>0 \quad \text { for } \quad \forall x \in \mathbb{R} .
$$

Thus $h \in \mathfrak{A}$. This means $h \in h_{\mathfrak{A}}^{s}$, i.e., $h_{\mathfrak{A}}^{s}$ is open in $h^{s}$. 


\subsection{Transformation}

\subsubsection{The diffeomorphism}

To study the moving boundary problem, in general, we usually first transform a moving boundary problem into a fixed boundary problem, then study the corresponding fixed boundary problem. So we now consider a reference domain $\Omega:=\mathbb{R} \times(0,1)$. In this chapter, we always consider $f \in h^{2+\alpha} \cap \widetilde{\mathfrak{A}}$, where $\alpha \in(0,1)$. Then we take $g=f-c \in$ $h_{\mathfrak{A}}^{2+\alpha}$, and define $\varphi_{g}: \Omega_{f} \rightarrow \Omega$ by

$$
\varphi_{g}\left(x^{\prime}, y^{\prime}\right):=\left(x^{\prime}, 1-\frac{y^{\prime}}{c+g\left(x^{\prime}\right)}\right), \quad\left(x^{\prime}, y^{\prime}\right) \in \Omega_{f},
$$

and the inverse function $\varphi_{g}^{-1}: \Omega \rightarrow \Omega_{f}$ by

$$
\varphi_{g}^{-1}(x, y):=(x,(1-y)(c+g(x))), \quad(x, y) \in \Omega
$$

Thus we can transform the moving domain $\Omega_{f}$ into the reference domain $\Omega$ by $\varphi_{g}$, (see Figure 2).

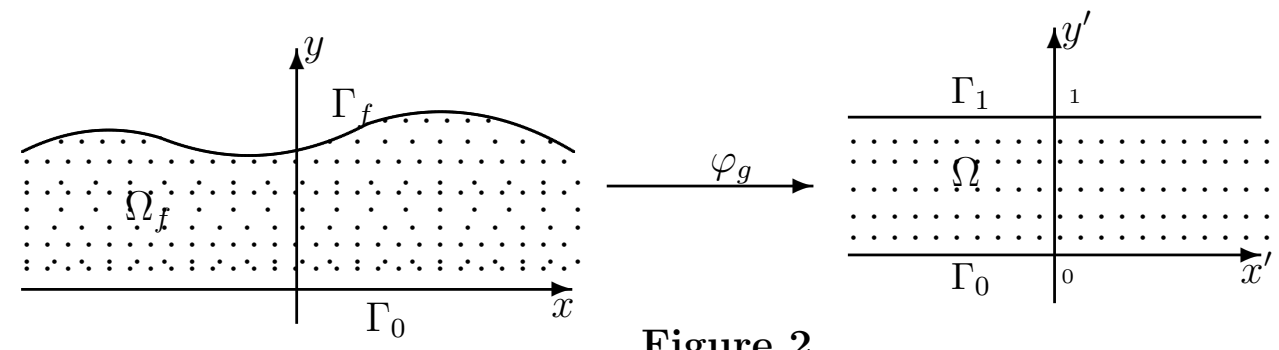

Figure 2

Lemma 4.3.1. Given $f \in B U C^{k}(\mathbb{R}), k \in \mathbb{N}$ with $\inf _{x \in \mathbb{R}}\{f(x)\}>0$, and $\phi_{f}(x, y):=$ $\left(x, 1-\frac{y}{f(x)}\right)$. Then we have

$$
\phi_{f} \in \operatorname{Diff} f^{k}\left(\Omega_{f}, \Omega\right)
$$

Proof It is obvious that $\phi_{f} \in C^{k}\left(\Omega_{f}\right)$ and

$$
D \phi_{f}(x, y)=\left(\begin{array}{cc}
1 & 0 \\
\frac{y f^{\prime}(x)}{f^{2}(x)} & \frac{-1}{f(x)}
\end{array}\right)
$$

Hence, by means of the assumption of $f$, $\operatorname{det}\left(D \phi_{f}\right)=-\frac{1}{f} \neq 0$, i.e. $\phi_{f}$ is a local $C^{k}$-diffeomorphism. Furthermore, observe that $y \mapsto 1-\frac{y}{f(x)}$ is strictly decreasing diffeomorphism. Thus this completes the proof. 


\subsubsection{The transformed operators}

Let

$$
\begin{aligned}
& \varphi_{*} u:=\varphi_{*}^{g} u:=u \circ \varphi_{g}^{-1} \quad \text { for } \quad u \in C\left(\overline{\Omega_{f}}\right) \text {, } \\
& \varphi^{*} v:=\varphi_{g}^{*} v:=v \circ \varphi_{g} \quad \text { for } \quad v \in C(\bar{\Omega}) \text {, }
\end{aligned}
$$

denote the push forward and pull back operators, respectively. Then we define the following transformed operators:

$$
\begin{array}{llc}
\mathcal{A}(g) v & := & -\varphi_{*}^{g} \Delta\left(\varphi_{g}^{*} v\right), \\
\mathcal{B}_{i}(g) v & := & \varphi_{*}^{g}\left(\gamma_{i}\left(\nabla\left(\varphi_{g}^{*} v\right) \mid n_{i}\right)\right) \quad i=0,1 .
\end{array}
$$

Here $(\cdot \mid \cdot)$ denotes inner product in $\mathbb{R}^{2}, \gamma_{i}: \Omega \rightarrow \Gamma_{i}(i=0,1)$ are trace operators, $\Gamma_{i}=$ $\mathbb{R} \times\{i\}, n_{0}=\left(-g_{x}, 1\right)$ and $n_{1}=(0,-1)$. Then problem (4.2.1)-(4.2.6) is transformed to

$$
\begin{aligned}
& \mathcal{A}(g) v=0 \quad \text { in } \quad J \times \Omega \text {, } \\
& v=g \text { on } J \times \Gamma_{0}, \\
& \mathcal{B}_{1}(g) v=\tilde{b}(g) \text { on } J \times \Gamma_{1} \text {, } \\
& \lim _{|z| \rightarrow \infty} v(t, z)=0 \text { on } J \text {, } \\
& \partial_{t} g+\mathcal{B}_{0}(g) v=0 \quad \text { on } \quad \dot{J} \times \Gamma_{0} \text {, } \\
& g(0, \cdot)=g_{0} \text { on } \mathbb{R},
\end{aligned}
$$

where $\tilde{b}(g):=-b(c+g)$ and $g_{0}(x):=f_{0}(x)-c$.

Proposition 4.3.2. The problem (4.2.1)-(4.2.6) and the problem (4.3.1)-(4.3.6) are equivalent.

Proof In fact, if $(u, f)$ is a solution of problem (4.2.1)-(4.2.6), then it is obvious that $\left(\varphi_{*} u-c, f-c\right)$ is a solution of problem (4.3.1)-(4.3.6). Hence it need to check that $\left(\varphi^{*} v+c, g+c\right)$ is a solution of problem $(4.2 .1)-(4.2 .6)$, if $(v, g)$ is a solution of problem (4.3.1)-(4.3.6). Therefore we only need to check equations (4.2.1), (4.2.3) and (4.2.5). Let $u:=\varphi^{*} v+c, f:=g+c$ and $(\xi, \eta)=\varphi_{g}(x, y)$. Clearly, $\xi=x$. Thanks to Lemma 4.3.1, we know $\varphi_{g} \in \operatorname{Diff}^{2}\left(\Omega_{f}, \Omega\right)$. Furthermore, deduced from (4.3.1), we get

$$
\Delta\left(\varphi^{*} v+c\right)=\Delta\left(\varphi_{g}^{*} v\right)=0 .
$$

It satisfies (4.2.1). Since

$$
\begin{aligned}
\left.\frac{\partial u}{\partial y}(x, y)\right|_{y=0} & =\left.\left(\nabla \varphi_{g}^{*} v \mid(0,1)\right)(x, y)\right|_{y=0}=-\left.\left(\nabla \varphi_{g}^{*} v \mid n_{1}\right)(x, y)\right|_{y=0} \\
& =-\left.\left(\nabla \varphi_{g}^{*} v \mid n_{1}\right) \varphi_{g}^{-1}(\xi, \eta)\right|_{\eta=1}=-\mathcal{B}_{1} v(\xi, \eta) \\
& =-\tilde{b}(g(\xi))=b(f(\xi))=b(f(x))
\end{aligned}
$$

Similarly, we can check that equation (4.2.5) holds. 
Lemma 4.3.3. Given $g \in \mathfrak{A}$, we have

$$
\mathcal{A}(g)=\sum_{j, k=1}^{2}-a_{j k}(g) \partial_{j} \partial_{k}+a_{2}(g) \partial_{2}, \quad \mathcal{B}_{i}(g)=\sum_{j=1}^{2} b_{j i}(g) \gamma_{i} \partial_{j}, i=0,1
$$

and

$$
\sum_{j, k=1}^{2} a_{j k}(g) \xi^{j} \xi^{k} \geq \underline{\alpha}(g)|\xi|^{2} \quad \text { for } \xi \in \mathbb{R}^{2}
$$

where $\pi(x, y):=1-y$ for $(x, y) \in \bar{\Omega}$ and

$$
\begin{array}{ll}
a_{11}(g):=1, & a_{12}(g):=a_{21}(g):=\frac{\pi g_{x}}{c+g}, \\
a_{22}(g):=\frac{1}{(c+g)^{2}}\left(1+\pi^{2} g_{x}^{2}\right), & a_{2}(g):=\frac{\pi}{c+g}\left(\frac{2 g_{x}^{2}}{c+g}-g_{x x}\right), \\
b_{10}:=-g_{x}, & b_{20}(g):=-\frac{1}{c+g}\left(1+g_{x}^{2}\right), \\
b_{11}(g):=0, & b_{21}(g):=\frac{1}{c+g}, \\
\underline{\alpha}(g):=\frac{1}{1+(c+g)^{2}+\pi^{2} g_{x}^{2}} . &
\end{array}
$$

Proof Let $\tilde{g}_{j k}:=\tilde{g}_{j k}(g):=\left(\partial_{j} \varphi_{g}^{-1} \mid \partial_{k} \varphi_{g}^{-1}\right), \quad 1 \leq j, k \leq 2$, denote the components of the metric tensor. Then

$$
\left[\tilde{g}_{j k}\right]=\left(\begin{array}{cc}
1+\pi^{2} g_{x}^{2} & -\pi(c+g) g_{x} \\
-\pi(c+g) g_{x} & (c+g)^{2}
\end{array}\right) .
$$

Moreover, it is easily verified that $\tilde{g}:=\operatorname{det}\left[\tilde{g}_{j k}\right]=(c+g)^{2}$ and that

$$
\left[\tilde{g}^{j k}\right]:=\left[\tilde{g}_{j k}\right]^{-1}=\left(\begin{array}{cc}
1 & \frac{\pi g_{x}}{c+g} \\
\frac{\pi g_{x}}{c+g} & \frac{1}{(c+g)^{2}}\left(1+\pi^{2} g_{x}^{2}\right)
\end{array}\right)
$$

Now, the assertion follows from the well-known formulas

$$
\begin{aligned}
& \mathcal{A}(g) v=-\varphi_{*}^{g} \Delta\left(\varphi_{g}^{*} v\right)=-\frac{1}{\sqrt{\tilde{g}}} \sum_{j, k=1}^{2} \partial_{j}\left(\sqrt{\tilde{g}} \tilde{g}^{j k} \partial_{k} v\right) \\
& \mathcal{B}_{i}(g) v=\varphi_{*}^{g}\left(\gamma_{i}\left(\nabla\left(\varphi_{g}^{*} v\right) \mid n_{i}\right)\right)=\varphi_{*}^{g}\left(\gamma_{i}\left[\sum_{k=1}^{2} \partial_{j} \varphi_{g}^{k} \partial_{k} v\right]_{1 \leq j \leq 2} \mid n_{i}\right), i=0,1 .
\end{aligned}
$$

Where $\varphi_{g}^{k}(1 \leq k \leq 2)$ denote the components of $\varphi_{g}$ i.e. $\varphi_{g}=\left(\varphi_{g}^{1}, \varphi_{g}^{2}\right)$.

To prove the second assertion, we fix $(x, y) \in \bar{\Omega}$ and suppress it in our notation. Set

$$
\underline{\alpha}:=\underline{\alpha}(g):=\left[1+(c+g)^{2}+\pi^{2} g_{x}^{2}\right]^{-1}, \quad \beta:=\beta(g):=2(c+g),
$$


and note that

$$
\underline{\alpha}^{-2}-\beta^{2}=\left[1-(c+g)^{2}-\pi^{2} g_{x}^{2}\right]^{2}+4 \pi^{2} g_{x}^{2} \geq 0 .
$$

Hence we find that $\underline{\alpha}^{2} \beta^{2} \leq 1$. This implies that

$$
0 \leq 1-\underline{\alpha}^{2} \beta^{2} \leq\left(1-\frac{\underline{\alpha}^{2} \beta^{2}}{2}\right)^{2}
$$

Observe that the smallest eigenvalue of $\left[\tilde{g}^{j k}\right]$ is given by

$$
\left.\lambda_{(} g\right)=\frac{1}{2(c+g)^{2}}\left\{1+(c+g)^{2}+\pi^{2} g_{x}^{2}-\sqrt{\left[1+(c+g)^{2}+\pi^{2} g_{x}^{2}\right]^{2}-4(c+g)^{2}}\right\} .
$$

Consequently,

$$
\left.\lambda_{(} g\right)=\frac{2}{\underline{\alpha} \beta^{2}}\left(1-\sqrt{1-\underline{\alpha}^{2} \beta^{2}}\right) \geq \frac{2}{\underline{\alpha} \beta^{2}}\left[1-\left(1-\frac{\underline{\alpha}^{2} \beta^{2}}{2}\right)\right]=\underline{\alpha},
$$

which completes the proof of Lemma 4.3.3.

In the following, we study problem (4.3.1)-(4.3.6) in the little Hölder spaces.

Lemma 4.3.4. Let $\alpha \in(0,1)$ be given. Then

$$
\left(\mathcal{A}(\cdot), \mathcal{B}_{0}(\cdot), \mathcal{B}_{1}(\cdot)\right) \in C^{\infty}\left(h_{\mathfrak{A}}^{2+\alpha}, \mathcal{L}\left(h^{2+\alpha}(\Omega), h^{\alpha}(\Omega) \times h^{1+\alpha} \times h^{1+\alpha}\right)\right),
$$

and

$$
\begin{aligned}
\partial \mathcal{A}(g)[h, v] & =\frac{2 \pi}{c+g}\left\{\left(\frac{g_{x} h}{c+g}-h_{x}\right) \partial_{1} \partial_{2} v+\left(\frac{h}{(c+g)^{2}}\left(\frac{1}{\pi}+\pi g_{x}^{2}\right)-\frac{\pi}{c+g} g_{x} h_{x}\right) \partial_{2}^{2} v\right. \\
& \left.-\left(\frac{2 g_{x}^{2} h}{(c+g)^{2}}-\frac{4 g_{x} h_{x}+g_{x x} h}{2(c+g)}+\frac{h_{x x}}{2}\right) \partial_{2} v\right\} \\
\partial \mathcal{B}_{0}(g)[h, v] & =-h_{x} \gamma_{0} \partial_{1} v+\frac{1}{c+g}\left(\frac{h+h g_{x}^{2}}{c+g}-2 g_{x} h_{x}\right) \gamma_{0} \partial_{2} v \\
\partial \mathcal{B}_{1}(g)[h, v] & =-\frac{h}{(c+g)^{2}} \gamma_{1} \partial_{2} v
\end{aligned}
$$

for $g \in h_{\mathfrak{A}}^{2+\alpha}, h \in h^{2+\alpha}$ and $v \in h^{2+\alpha}(\Omega)$.

Proof It is obvious that the first assertion holds. Given $g \in h_{\mathfrak{A}}^{2+\alpha}$. For $h \in h^{2+\alpha}$ and 
$v \in h^{2+\alpha}(\Omega)$, we have

$$
\begin{aligned}
\partial \mathcal{A}(g)[h, v]=\lim _{\varepsilon \rightarrow 0} & \frac{\mathcal{A}(g+\varepsilon h) v-\mathcal{A}(g) v}{\varepsilon} \\
=\lim _{\varepsilon \rightarrow 0} \frac{1}{\varepsilon}\left[-\partial_{1}^{2} v-\frac{2 \pi\left(g_{x}+\varepsilon h_{x}\right)}{c+g+\varepsilon h} \partial_{1} \partial_{2} v-\frac{1+\pi^{2}\left(g_{x}+\varepsilon h_{x}\right)^{2}}{(c+g+\varepsilon h)^{2}} \partial_{2}^{2} v\right. & \\
& +\frac{\pi}{c+g+\varepsilon h}\left(\frac{2\left(g_{x}+\varepsilon h_{x}\right)^{2}}{c+g+\varepsilon h}-\left(g_{x x}+\varepsilon h_{x x}\right)\right) \partial_{2} v+\partial_{1}^{2} v \\
& \left.+\frac{2 \pi g_{x}}{c+g} \partial_{1} \partial_{2} v+\frac{1+\pi^{2} g_{x}^{2}}{(c+g)^{2}} \partial_{2}^{2} v-\frac{\pi}{c+g}\left(\frac{2 g_{x}^{2}}{c+g}-g_{x x}\right) \partial_{2} v\right] \\
= & \lim _{\varepsilon \rightarrow 0} \frac{1}{\varepsilon}\left[\frac{2 \pi\left(g_{x} \varepsilon h-(c+g) \varepsilon h_{x}\right)}{(c+g)(c+g+\varepsilon h)} \partial_{1} \partial_{2} v\right. \\
& \quad+\frac{\left(1+\pi^{2} g_{x}^{2}\right) \varepsilon h(2(c+g)+\varepsilon h)-(c+g)^{2} \pi^{2} \varepsilon h_{x}\left(2 g_{x}+\varepsilon h_{x}\right)}{(c+g)^{2}(c+g+\varepsilon h)^{2}} \partial_{2}^{2} v \\
& +\frac{\pi}{c+g+\varepsilon h}\left(\frac{2\left((c+g)^{2} \varepsilon h_{x}\left(2 g_{x}+\varepsilon h_{x}\right)-g_{x}^{2} \varepsilon h(2(c+g)+\varepsilon h)\right)}{(c+g)^{2}(c+g+\varepsilon h)}\right. \\
& +\frac{2 \pi\left(g_{x} h-(c+g) h_{x}\right)}{(c+g)^{2}} \partial_{1} \partial_{2} v+\frac{2 h\left(1+\pi^{2} g_{x}^{2}\right)-2 \pi^{2}(c+g) g_{x} h_{x}}{(c+g)_{2}^{2} v} \\
& +\frac{\pi}{c+g}\left(\frac{4 g_{x} h_{x}}{c+g}-\frac{4 g_{x}^{2} h}{(c+g)^{2}}+\frac{g_{x x} h}{c+g}-h_{x x}\right) \partial_{2} v .
\end{aligned}
$$

Similarly, the other assertions hold.

\subsubsection{Isomorphisms induced by elliptic boundary value prob- lems}

In the following, we shall introduce an elliptic regularity theorem.

Theorem 4.3.5. a) Let $g \in h_{\mathfrak{A}}^{2+\alpha}, \lambda \geq 0$ and $\mu>0$ be given. Then

$$
\begin{gathered}
\left(\lambda+\mathcal{A}(g), \gamma_{0},(c+g) \mathcal{B}_{1}(g)\right) \in I \operatorname{som}\left(h^{2+\alpha}(\Omega), h^{\alpha}(\Omega) \times h^{2+\alpha} \times h^{1+\alpha}\right), \\
\left(\lambda+\mathcal{A}(g), \mu \gamma_{0}+\mathcal{B}_{0}(g), \mathcal{B}_{1}(g)\right) \in I \operatorname{som}\left(h^{2+\alpha}(\Omega), h^{\alpha}(\Omega) \times h^{1+\alpha} \times h^{1+\alpha}\right) .
\end{gathered}
$$

b) Given $\lambda_{0} \geq 0$ and $g \in h_{\mathfrak{A}}^{2+\alpha}$, there exists a positive constant $C$, depending on $\|g\|_{h^{2+\alpha}}$, $\lambda_{0}, \alpha$ and $c$ such that

$$
\|u\|_{h^{2+\alpha}(\Omega)} \leq C\left(\|(\lambda+\mathcal{A}(g)) u\|_{h^{\alpha}(\Omega)}+\left\|\gamma_{0} u\right\|_{h^{2+\alpha}}+\left\|(c+g) \mathcal{B}_{1}(g) u\right\|_{h^{1+\alpha}}\right)
$$

for all $u \in h^{2+\alpha}(\Omega)$ and $\lambda \in\left[0, \lambda_{0}\right]$.

Proof The proof is based on the classical estimates of Agmon-Douglis-Nirenberg [2] for elliptic equations, see also the Theorem 3.5 in [12]. 
Remark 4.3.6. Theorem 4.3.5 ensures that the following problem: given $g \in h_{\mathfrak{A}}^{2+\alpha}$ and $\lambda \geq 0$

$$
\begin{cases}\lambda v+\mathcal{A}(g) v=v_{1} & \text { in } \Omega, \\ \gamma_{0} v=v_{2} & \text { on } \Gamma_{0}, \\ (c+g) \mathcal{B}_{1}(g) v=v_{3} & \text { on } \Gamma_{1},\end{cases}
$$

for every $\left(v_{1}, v_{2}, v_{3}\right) \in h^{\alpha}(\Omega) \times h^{2+\alpha} \times h^{1+\alpha}$, there exists a unique solution $v \in h^{2+\alpha}(\Omega)$

In particular, given $g \in h_{\mathfrak{A}}^{2+\alpha}$, thanks to Remark 4.3.6, we can define

$$
\mathcal{R}(g):=\left(\mathcal{A}(g), \gamma_{0},(c+g) \mathcal{B}_{1}(g)\right)^{-1}
$$

and its restrictions

$$
\begin{aligned}
& \mathcal{S}(g):=\mathcal{R}(g) \mid h^{\alpha}(\Omega) \times\{0\} \times\{0\} \\
& \mathcal{T}(g):=\mathcal{R}(g) \mid\{0\} \times h^{2+\alpha} \times\{0\} \\
& \mathcal{U}(g):=\mathcal{R}(g) \mid\{0\} \times\{0\} \times h^{1+\alpha}
\end{aligned}
$$

Obviously, let $v_{1}:=\mathcal{T}(g) h_{1}, v_{2}:=\mathcal{U}(g) h_{2}$ for $h_{1} \in h^{2+\alpha}, h_{2} \in h^{1+\alpha}$, then we get

$$
\begin{aligned}
& \mathcal{A}(g) v_{1}=0 \quad \text { in } \Omega, \quad \gamma_{0} v_{1}=h_{1} \quad \text { on } \Gamma_{0}, \quad \mathcal{B}_{1}(g) v_{1}=0 \quad \text { on } \Gamma_{1} \text {; } \\
& \mathcal{A}(g) v_{2}=0 \quad \text { in } \Omega, \quad \gamma_{0} v_{2}=0 \quad \text { on } \Gamma_{0}, \quad(c+g) \mathcal{B}_{1}(g) v_{2}=h_{2} \quad \text { on } \Gamma_{1} \text {. }
\end{aligned}
$$

Lemma 4.3.7. We have

$$
\mathcal{T}(\cdot) \in C^{\infty}\left(h_{\mathfrak{A}}^{2+\alpha}, \mathcal{L}\left(h^{2+\alpha}, h^{2+\alpha}(\Omega)\right)\right), \quad \mathcal{U}(\cdot) \in C^{\infty}\left(h_{\mathfrak{A}}^{2+\alpha}, \mathcal{L}\left(h^{1+\alpha}, h^{2+\alpha}(\Omega)\right)\right)
$$

and

$$
\partial \mathcal{T}(g)[h, \cdot]=-\mathcal{S}(g) \partial \mathcal{A}(g)[h, \mathcal{T}(g) \cdot], \quad \partial \mathcal{U}(g)[h, \cdot]=-\mathcal{S}(g) \partial \mathcal{A}(g)[h, \mathcal{U}(g) \cdot]
$$

for $g \in h_{\mathfrak{A}}^{2+\alpha}$, and $h \in h^{2+\alpha}$.

Proof For simplicity, let $X:=h_{\mathfrak{A}}^{2+\alpha}, F_{i}:=h^{i+\alpha}(\Omega)(i=0,1,2), E_{j}:=h^{i+\alpha}(j=1,2)$.

a) Thanks to Remark 4.2.1, we know that $X$ is an open subset of $E_{2}$. Moreover, letting

$$
A(g):=\left(\mathcal{A}(g), \gamma_{0},(c+g) \mathcal{B}_{1}(g)\right), \quad g \in X .
$$

By means of Lemma 4.3.4 and Theorem 4.3.5, we also know that

$$
A \in C^{\infty}\left(X, \operatorname{Isom}\left(F_{2}, F_{0} \times E_{2} \times E_{1}\right)\right)
$$

with (note that $(c+g) \mathcal{B}_{1}(g)=\gamma_{1} \partial_{2}$ is independent of $g$ )

$$
\partial A(g) h=(\partial \mathcal{A}(g) h, 0,0) \text { for } h \in E_{2} .
$$

b) Given $A \in I \operatorname{som}\left(F_{2}, F_{0} \times E_{2} \times E_{1}\right)$, define $j(A):=A^{-1}$. Since $I \operatorname{som}\left(F_{2}, F_{0} \times E_{2} \times E_{1}\right)$ is open in $\mathcal{L}\left(F_{2}, F_{0} \times E_{2} \times E_{1}\right)$, then

$$
j \in C^{\infty}\left(I \operatorname{som}\left(F_{2}, F_{0} \times E_{2} \times E_{1}\right), \mathcal{L}\left(F_{0} \times E_{2} \times E_{1}, F_{2}\right)\right),
$$


with

$$
\partial j(A) \tilde{A}=-A^{-1} \tilde{A} A^{-1}
$$

for $A \in \operatorname{Isom}\left(F_{2}, F_{0} \times E_{2} \times E_{1}\right)$ and $\tilde{A} \in \mathcal{L}\left(F_{2}, F_{0} \times E_{2} \times E_{1}\right)$.

c) Let $R \in \mathcal{L}\left(F_{0} \times E_{2} \times E_{1}, F_{2}\right)$ be given, and define $p(R) \in \mathcal{L}\left(E_{2}, F_{2}\right)$ by

$$
p(R) h:=R(0, h, 0) \text { for } h \in E_{2} .
$$

Then $p \in \mathcal{L}\left(\mathcal{L}\left(F_{0} \times E_{2} \times E_{1}, F_{2}\right), \mathcal{L}\left(E_{2}, F_{2}\right)\right)$ and consequently

$$
\partial p(R) S=p(S) \text { for } R, S \in \mathcal{L}\left(F_{0} \times E_{2} \times E_{1}, F_{2}\right) .
$$

So that $\mathcal{T}=p \circ j \circ A$ and

$$
\begin{aligned}
\partial \mathcal{T}(g) h & =\partial(p \circ j \circ A(g)) h \\
& =\partial p(j(A(g))) \partial j(A(g)) \partial A(g) h \\
& =\partial p(j(A(g))) \partial j(A(g))(\partial \mathcal{A}(g) h, 0,0) \\
& =\partial p(j(A(g)))\left(-A^{-1}(g)(\partial \mathcal{A}(g) h, 0,0) A^{-1}(g)\right) \\
& =p\left(-A^{-1}(g)(\partial \mathcal{A}(g) h, 0,0) A^{-1}(g)\right),
\end{aligned}
$$

i.e.

$$
\begin{aligned}
\partial \mathcal{T}(g)[h, v] & =p\left(-A^{-1}(g)(\partial \mathcal{A}(g) h, 0,0) A^{-1}(g)\right) v \\
& =-A^{-1}(g)(\partial \mathcal{A}(g) h, 0,0) A^{-1}(g)(0, v, 0) \\
& =-\mathcal{R}(g)(\partial \mathcal{A}(g) h, 0,0) \mathcal{T}(g) v \\
& =-\mathcal{R}(g)(\partial \mathcal{A}(g)[h, \mathcal{T}(g) v], 0,0) \\
& =-\mathcal{S}(g) \partial \mathcal{A}(g)[h, \mathcal{T}(g) v]
\end{aligned}
$$

for $h, v \in E_{2}$.

It is similar to $\partial \mathcal{U}(g) h$, thus we can get

$$
\partial \mathcal{U}(g)[h, \cdot]=-\mathcal{S}(g) \partial \mathcal{A}(g)[h, \mathcal{U}(g) \cdot] .
$$

\subsection{Reduced equation}

\subsubsection{Equivalent formulation}

In this subsection, we shall show that problem (4.3.1)-(4.3.6) can be reduced to an evolution problem.

Given $g \in h_{\mathfrak{A}}^{2+\alpha}$, we define

$$
\Phi_{\widehat{b}}(g):=\mathcal{B}_{0}(g)[\mathcal{T}(g) g+\mathcal{U}(g) \widehat{b}(g)] .
$$


Here $\widehat{b}(g):=(c+g) \tilde{b}(g)=-(c+g) b(c+g)$. Suppose that $g_{0} \in h_{\mathfrak{A}}^{2+\alpha}$. A function $g: J \rightarrow h^{1+\alpha}$ is said to be a classical solution of

$$
\dot{g}+\Phi_{\widehat{b}}(g)=0, \quad g(0)=g_{0},
$$

iff $g \in C\left(J, h_{\mathfrak{A}}^{2+\alpha}\right) \cap C^{1}\left(J, h^{1+\alpha}\right)$ and $g$ satisfies (4.4.1) pointwise.

Proposition 4.4.1. Let $g_{0} \in h_{\mathfrak{A}}^{2+\alpha}$ be given.

i) Suppose that $g$ is a classical solution of problem (4.4.1) on J. Let $v(t):=\mathcal{T}(g(t)) g(t)$ $+\mathcal{U}(g(t)) \widehat{b}(g(t))$. Then the pair $(v, g)$ is a classical solution of problem (4.3.1)-(4.3.6) on $J$, having the additional regularity

$$
g \in C\left(J, h_{\mathfrak{A}}^{2+\alpha}\right) \cap C^{1}\left(J, h^{1+\alpha}\right), \quad v(t, \cdot) \in h^{2+\alpha}(\Omega), t \in J .
$$

ii) Suppose that $(v, g)$ is a classical solution of problem (4.3.1)-(4.3.6) on J, having the regularity (4.4.2). Then $g$ is a classical solution of problem (4.4.1) on J.

Proof If $g$ is a classical solution of (4.4.1), then let $v=\mathcal{T}(g) g+\mathcal{U}(g) \widehat{b}(g)$, we can check that $(v, g)$ is a classical solution of (4.3.1)-(4.3.6).

If $(v, g)$ is a classical solution of (4.3.1)-(4.3.6), by the uniqueness, then we know that

$$
v=\mathcal{T}(g) g+\mathcal{U}(g) \widehat{b}(g) .
$$

Thus, $g$ is a classical solution of (4.4.1).

Corollary 4.4.2. $\Phi_{\widehat{b}} \in C^{\infty}\left(h_{\mathfrak{A}}^{2+\alpha}, h^{1+\alpha}\right)$ and

$$
\begin{aligned}
\partial \Phi_{\widehat{b}}(g) h & =\partial \mathcal{B}_{0}(g)[h, \mathcal{T}(g) g+\mathcal{U}(g) \widehat{b}(g)]+\mathcal{B}_{0}(g) \mathcal{T}(g) h+\mathcal{B}_{0}(g) \mathcal{U}(g) \widehat{b}^{\prime}(g) h \\
& -\mathcal{B}_{0}(g) \mathcal{S}(g) \partial \mathcal{A}(g)[h, \mathcal{T}(g) g]-\mathcal{B}_{0}(g) \mathcal{S}(g) \partial \mathcal{A}(g)[h, \mathcal{U}(g) \widehat{b}(g)]
\end{aligned}
$$

for $g \in h_{\mathfrak{A}}^{2+\alpha}$ and $h \in h^{2+\alpha}$.

Proof By means of Lemma 4.3.4 and Lemma 4.3.7, we know

$$
\begin{aligned}
& \mathcal{B}_{0}(\cdot) \in C^{\infty}\left(h_{\mathfrak{A}}^{2+\alpha}, \mathcal{L}\left(h^{2+\alpha}(\Omega), h^{1+\alpha}\right)\right), \\
& \mathcal{T}(\cdot) \in C^{\infty}\left(h_{\mathfrak{A}}^{2+\alpha}, \mathcal{L}\left(h^{2+\alpha}, h^{2+\alpha}(\Omega)\right)\right), \\
& \mathcal{U}(\cdot) \in C^{\infty}\left(h_{\mathfrak{A}}^{2+\alpha}, \mathcal{L}\left(h^{1+\alpha}, h^{2+\alpha}(\Omega)\right)\right) .
\end{aligned}
$$

Then, since $\widehat{b}$ is smooth, for $\forall g \in h_{\mathfrak{A}}^{2+\alpha}$, we get

$$
\mathcal{T}(g) g \in h^{2+\alpha}(\Omega), \quad \mathcal{U}(g) \widehat{b}(g) \in h^{2+\alpha}(\Omega) \quad \text { and } \quad \mathcal{B}_{0}(g) \in \mathcal{L}\left(h^{2+\alpha}(\Omega), h^{1+\alpha}\right) .
$$

Thus, by definition, $\Phi_{\widehat{b}}(g) \in h^{1+\alpha}$, i.e., $\Phi_{\widehat{b}}(g): h_{\mathfrak{A}}^{2+\alpha} \rightarrow h^{1+\alpha}$. Further, we also get that

$$
\Phi_{\widehat{b}} \in C^{\infty}\left(h_{\mathfrak{A}}^{2+\alpha}, h^{1+\alpha}\right) .
$$

Then $\partial \Phi_{\widehat{b}}(g) \in \mathcal{L}\left(h^{2+\alpha}, h^{1+\alpha}\right)$. Using Lemma 4.3.4 and Lemma 4.3.7 again, by chain law, we obtain

$$
\begin{aligned}
\partial \Phi_{\widehat{b}}(g) h & =\partial \mathcal{B}_{0}(g)[h, \mathcal{T}(g) g+\mathcal{U}(g) \widehat{b}(g)]+\mathcal{B}_{0}(g) \mathcal{T}(g) h+\mathcal{B}_{0}(g) \mathcal{U}(g) \widehat{b}^{\prime}(g) h \\
& -\mathcal{B}_{0}(g) \mathcal{S}(g) \partial \mathcal{A}(g)[h, \mathcal{T}(g) g]-\mathcal{B}_{0}(g) \mathcal{S}(g) \partial \mathcal{A}(g)[h, \mathcal{U}(g) \widehat{b}(g)]
\end{aligned}
$$

for $g \in h_{\mathfrak{A}}^{2+\alpha}$ and $h \in h^{2+\alpha}$. 


\subsubsection{The linearization}

For simplicity, we always assume that

$$
\widehat{b}(0)=0 .
$$

Thanks to Proposition 4.4.1, we only need to study problem (4.4.1). By (4.4.3), we know that $g=0$ is the stationary solution of (4.4.1). To study problem (4.4.1), using the theory of Section 3.3, we first consider its corresponding linear problem: For a suitable open set $\mathcal{O} \subset h_{\mathfrak{A}}^{2+\alpha}$ such that $g_{0} \in \mathcal{O}$,

$$
g^{\prime}+\partial \Phi_{\widehat{b}}(\bar{g}) g=G(g, \bar{g}),
$$

where $\bar{g} \in \mathcal{O}$ and $G(g, \bar{g})=\partial \Phi_{\widehat{b}}(\bar{g}) g-\Phi_{\widehat{b}}(g)$.

Since we are concerning about the behavior near $g=0$, we take $\bar{g} \equiv 0$. This means that we shall study the following problem

$$
g^{\prime}+\partial \Phi_{\widehat{b}}(0) g=G(g) ; \quad g(0)=g_{0},
$$

here $G(g)=\partial \Phi_{\widehat{b}}(0) g-\Phi_{\widehat{b}}(g)$. Before studying (4.4.5), we first study the operator $\partial \Phi_{\widehat{b}}(0)$. By Corollary 4.4.2, we get

$$
\partial \Phi_{\widehat{b}}(0) h=\mathcal{B}_{0}(0) \mathcal{T}(0) h+\mathcal{B}_{0}(0) \mathcal{U}(0) \widehat{b}^{\prime}(0) h .
$$

\subsubsection{Fourier multipliers}

In the following we shall express the operator $\partial \Phi_{\widehat{b}}(0)$ as a Fourier multiplier.

Lemma 4.4.3. For $h \in h^{2+\alpha}$, we have

$$
\begin{aligned}
& \mathcal{T}(0) h=\mathcal{F}^{-1}\left(\frac{\cosh (c|\xi|(1-y))}{\cosh (c|\xi|)} \mathcal{F} h\right), \\
& \mathcal{U}(0) h=\mathcal{F}^{-1}\left(\frac{\sinh (c|\xi| y)}{c|\xi| \cosh (c|\xi|)} \mathcal{F} h\right) .
\end{aligned}
$$

Proof In fact, we consider two problems

$$
\mathcal{A}(0) v_{1}=0 \text { in } \Omega, \quad v_{1}=h_{1} \text { on } \Gamma_{0}, \quad B_{1}(0) v_{1}=0 \text { on } \Gamma_{1},
$$

and

$$
\mathcal{A}(0) v_{2}=0 \text { in } \Omega, \quad v_{2}=0 \text { on } \Gamma_{0}, \quad c B_{1}(0) v_{2}=h_{2} \text { on } \Gamma_{1} .
$$

Here $\mathcal{A}(0)=-\partial_{1}^{2}-\frac{1}{c^{2}} \partial_{2}^{2}, \mathcal{B}_{1}(0)=\frac{1}{c} \gamma_{1} \partial_{2}$.

First, we consider $\mathcal{A}(0) v=0$. By Fourier transformation with respect to $x$, we have

$$
\mathcal{F} v(\xi, y)=C_{1} e^{c|\xi| y}+C_{2} e^{-c|\xi| y}, \quad C_{1}, C_{2} \text { are two undetermined constants. }
$$


i) For $v_{1}$, we have

$$
\begin{array}{r}
C_{1}+C_{2}=\mathcal{F} h_{1}, \\
C_{1}|\xi| e^{c|\xi|}-C_{2}|\xi| e^{-c|\xi|}=0 .
\end{array}
$$

Then

$$
C_{1}=\frac{\mathcal{F} h_{1}}{1+e^{2 c|\xi|}}, \quad C_{2}=e^{2 c|\xi|} \frac{\mathcal{F} h_{1}}{1+e^{2 c|\xi|}} .
$$

Therefore,

$$
\mathcal{F} v_{1}=\frac{\cosh (c|\xi|(1-y))}{\cosh (c|\xi|)} \mathcal{F} h_{1} .
$$

Since $v_{1}=\mathcal{T}(0) h_{1}$ is a solution of problem (4.4.7), we have

$$
\mathcal{T}(0)=\mathcal{F}^{-1} \frac{\cosh (c|\xi|(1-y))}{\cosh (c|\xi|)} \mathcal{F} .
$$

ii) For $v_{2}$,we have

$$
\begin{array}{r}
C_{1}+C_{2}=0, \\
c C_{1}|\xi| e^{c|\xi|}-c C_{2}|\xi| e^{-c|\xi|}=\mathcal{F} h_{2} .
\end{array}
$$

Then

$$
C_{1}=\frac{\mathcal{F} h_{2}}{2 c|\xi| \cosh (c|\xi|)}, \quad C_{2}=-\frac{\mathcal{F} h_{2}}{2 c|\xi| \cosh (c|\xi|)} .
$$

Therefore,

$$
\mathcal{F} v_{2}=\frac{\sinh (c|\xi| y)}{c|\xi| \cosh (c|\xi|)} \mathcal{F} h_{2}
$$

Since $v_{2}=\mathcal{U}(0) h_{2}$ is a solution of problem (4.4.8), we have

$$
\mathcal{U}(0)=\mathcal{F}^{-1} \frac{\sinh (c|\xi| y)}{c|\xi| \cosh (c|\xi|)} \mathcal{F} .
$$

Since $B_{0}(0)=-\frac{1}{c} \gamma_{0} \partial_{2}$, by Lemma 4.4.3 and (4.4.6), it is easy to obtain that

$$
\partial \Phi_{\widehat{b}}(0) h=\mathcal{F}^{-1} \frac{c|\xi| \sinh (c|\xi|)-\widehat{b}^{\prime}(0)}{c \cosh (c|\xi|)} \mathcal{F} h, \quad \forall h \in h^{2+\alpha} .
$$

\subsection{Sectorial operator}

In the section, we shall investigate the operator $A_{\widehat{b}}:=\partial \Phi_{\widehat{b}}(0): h^{2+\alpha} \subset h^{1+\alpha} \rightarrow h^{1+\alpha}$, and show $-A_{\widehat{b}}$ is a sectorial operator. Thanks to Proposition 3.1.15, we need to know that the spectrum and estimate of $-A_{\widehat{b}}$. 


\subsubsection{Spectral properties}

In the subsection, we shall investigate the spectrum of the operator $A_{\widehat{b}}$. To do it, we first shall study the spectrum of $A_{\widehat{b}}$ in $L_{2}(\mathbb{R})$, then in Sobolev spaces $H^{s}(\mathbb{R}), s>0$. Finally, we use Sobolev's embedding theorem to get our results.

By Corollary 4.4.2 and (4.4.9), we have

$$
A_{\widehat{b}} \in \mathcal{L}\left(h^{2+\alpha}, h^{1+\alpha}\right),
$$

and

$$
A_{\widehat{b}}=\mathcal{F}^{-1} a_{\widehat{b}}(\xi) \mathcal{F}
$$

where

$$
a_{\widehat{b}}(\xi)=\frac{c|\xi| \sinh (c|\xi|)-\widehat{b}^{\prime}(0)}{c \cosh (c|\xi|)}=\frac{c \xi \sinh (c \xi)-\widehat{b}^{\prime}(0)}{c \cosh (c \xi)} .
$$

For simplicity, we assume that $\widehat{b}^{\prime}(0)<0$. By the definition of $a_{\widehat{b}}(\xi)$, we know that $a_{\widehat{b}}(\xi)$ is an even function, $a_{\widehat{b}}(\xi)>0$ for $\xi \in \mathbb{R}$ and $\lim _{\xi \rightarrow+\infty} a_{\widehat{b}}(\xi)=+\infty$. Thus there exists a constant $\omega_{0}>0$, such that $\omega_{0}=\min _{\xi \in \mathbb{R}}\left\{a_{\widehat{b}}(\xi)\right\}$.

First, we consider an auxiliary problem, i.e. studying $A_{\widehat{b}}$ on $L_{2}(\mathbb{R})$.

Lemma 4.5.1. $A_{\widehat{b}}$ is self-adjoint on $L_{2}(\mathbb{R})$, and

$$
\left(A_{\widehat{b}} u, u\right)_{L_{2}} \geq \omega_{0}\|u\|_{2}^{2}, \quad \text { for } u \in L_{2}(\mathbb{R}) .
$$

Proof For every $u, v \in L_{2}(\mathbb{R})$, thanks to Plancherel's theorem, i.e.

$$
(u, v)_{L_{2}}=(\mathcal{F} u, \mathcal{F} v)_{L_{2}},
$$

we have

$$
\begin{aligned}
\left(u, A_{\widehat{b}}^{*} v\right)_{L_{2}}=\left(A_{\widehat{b}} u, v\right)_{L_{2}} & =\left(\mathcal{F}^{-1} a_{\widehat{b}}(\xi) \mathcal{F} u, v\right)_{L_{2}}=\left(a_{\widehat{b}}(\xi) \mathcal{F} u, \mathcal{F} v\right)_{L_{2}} \\
& =\left(\mathcal{F} u, \overline{a_{\widehat{b}}(\xi)} \mathcal{F} v\right)_{L_{2}}=\left(u, \mathcal{F}^{-1} \overline{a_{\widehat{b}}(\xi)} \mathcal{F} v\right)_{L_{2}}
\end{aligned}
$$

Since $\overline{a_{\widehat{b}}(\xi)}=a_{\widehat{b}}(\xi)$, we can get that

$$
A_{\widehat{b}}^{*}=\mathcal{F}^{-1} \overline{a_{\widehat{b}}(\xi)} \mathcal{F}=A_{\widehat{b}},
$$

i.e. $A_{\widehat{b}}$ is self-adjoint.

Moreover, for every $u \in L_{2}(\mathbb{R})$, we get that

$$
\begin{aligned}
\left(A_{\widehat{b}} u, u\right)_{L_{2}} & =\left(a_{\widehat{b}}(\xi) \mathcal{F} u, \mathcal{F} u\right)_{L_{2}}=\int_{\mathbb{R}} a_{\widehat{b}}(\xi)|\mathcal{F} u|^{2} d \xi \\
& \geq \omega_{0} \int_{\mathbb{R}}|\mathcal{F} u|^{2} d \xi=\omega_{0}\|u\|_{2}^{2} .
\end{aligned}
$$


Proposition 4.5.2.

$$
\rho\left(A_{\hat{b}}\right) \supseteq\left\{\lambda \in \mathbb{C} ; \operatorname{Re} \lambda<\omega_{0}\right\} .
$$

Proof For every $\lambda \in\left\{\lambda \in \mathbb{C} ; \operatorname{Re} \lambda<\omega_{0}\right\}$, since $a_{\widehat{b}}(\xi) \geq \omega_{0}$, we get $\left|\lambda-a_{\widehat{b}}(\xi)\right| \geq$ $\left|\lambda-\omega_{0}\right|$. By means of Plancherel's theorem, we have

$$
\begin{aligned}
\left\|\left(\lambda-A_{\widehat{b}}\right) u\right\|_{2}^{2} & =\left(\left(\lambda-A_{\widehat{b}}\right) u,\left(\lambda-A_{\widehat{b}}\right) u\right)_{L_{2}} \\
& =\left(\mathcal{F}^{-1}\left(\lambda-a_{\widehat{b}}(\xi)\right) \mathcal{F} u, \mathcal{F}^{-1}\left(\lambda-a_{\hat{b}}\right)(\xi) \mathcal{F} u\right)_{L_{2}} \\
& =\left(\left(\lambda-a_{\widehat{b}}(\xi)\right) \mathcal{F} u,\left(\lambda-a_{\widehat{b}}(\xi)\right) \mathcal{F} u\right)_{L_{2}} \\
& =\int_{\mathbb{R}}\left|\lambda-a_{\widehat{b}}(\xi)\right|^{2}|\mathcal{F} u|^{2} d \xi \\
& \geq\left|\lambda-\omega_{0}\right|^{2}\|u\|_{2}^{2} .
\end{aligned}
$$

Hence $\lambda-A_{\widehat{b}}$ is injective. Now we will prove that $\lambda-A_{\widehat{b}}$ is surjective. Suppose $u \in$ Range $\left(\lambda-A_{\widehat{b}}\right)^{\perp}$, then, by Lemma 4.5.1, we have

$$
0=\left|\left(\left(\lambda-A_{\hat{b}}\right) u, u\right)_{L_{2}}\right|=\left|\lambda\|u\|_{2}^{2}-\left(A_{\widehat{b}} u, u\right)_{L_{2}}\right| \geq\left|\lambda-\omega_{0}\right|\|u\|_{2}^{2} .
$$

Thus, $u=0$, i.e. $\lambda-A_{\widehat{b}}$ is surjective. Hence $\left(\lambda-A_{\hat{b}}\right)^{-1}$ exists. From (4.5.2), we obtain that $\left(\lambda-A_{\hat{b}}\right)^{-1}$ is bounded. This means that $\lambda \in \rho\left(A_{\hat{b}}\right)$.

Second, we consider the operator $A_{\widehat{b}}$ on the Sobolev Space $H^{s}:=H^{s}(\mathbb{R})(s$ is an arbitrary positive real number). The inner product of $H^{s}$ is expressed by

$$
(u, v)_{H^{s}}=\left(\Lambda^{s} \mathcal{F} u, \Lambda^{s} \mathcal{F} v\right)_{L_{2}} \quad \text { for } \quad u, v \in H^{s},
$$

here $\Lambda^{s}=\left(1+\xi^{2}\right)^{s / 2}$. Similarly, we can also get that $A_{\widehat{b}}$ is self-adjoint and

$$
\rho\left(A_{\hat{b}}\right) \supseteq\left\{z \in \mathbb{C} ; \operatorname{Re}(z)<\omega_{0}\right\} .
$$

In the following, we will introduce the Sobolev's embedding theorem (cf. [28]).

Theorem 4.5.3 (Sobolev's Embedding Theorem). If $s>k+\frac{n}{2}$, then

$$
H^{s}\left(\mathbb{R}^{n}\right) \hookrightarrow B U C^{k}\left(\mathbb{R}^{n}\right) .
$$

Lemma 4.5.4. Let $E, F$ be Banach spaces, and $X$ be a topological space. Further assume that $X \hookrightarrow E \hookrightarrow F$. If $X$ is dense in $E$, then $X$ and $E$ have the same closure in $F$, that is, $\bar{X}=\bar{E}$ in $F$.

Proof Thank to the assumption, it is clear that $\bar{X} \subset \bar{E}$. Thus it is sufficient to prove $\bar{E} \subset \bar{X}$. For $\forall u \in \bar{E}$ and $\varepsilon>0$, there exists some $u_{0} \in E$ such that

$$
\left\|u-u_{0}\right\|_{F}<\frac{\varepsilon}{2}
$$


Since $E \hookrightarrow F$, there exists a positive constant $C$ such that

$$
\|v\|_{F} \leq C\|v\|_{E} \quad \text { for } v \in E .
$$

Furthermore since $X$ is dense in $E$, there also exists some $u_{1} \in X$ such that

$$
\left\|u_{0}-u_{1}\right\|_{E}<\frac{\varepsilon}{2 C}
$$

Hence,

$$
\left\|u-u_{1}\right\|_{F} \leq\left\|u-u_{0}\right\|_{F}+\left\|u_{0}-u_{1}\right\|_{F}<\frac{\varepsilon}{2}+C\left\|u_{0}-u_{1}\right\|_{E}<\varepsilon .
$$

This means $u \in \bar{X}$.

Corollary 4.5.5. For $s>p>0, h^{s} \stackrel{d}{\hookrightarrow} h^{p}$.

Proof It is easy to know that

$$
\mathcal{S}(\mathbb{R}) \stackrel{d}{\hookrightarrow} h^{s} \hookrightarrow B U C^{p}(\mathbb{R}) .
$$

Thanks to Lemma 4.5.4, we get

$$
\overline{h^{s}}=\overline{\mathcal{S}(\mathbb{R})}=h^{p} \text { in } B U C^{p}(\mathbb{R}) .
$$

This means $h^{s}$ is dense in $h^{p}$.

Corollary 4.5.6. If $s>k+\frac{3}{2}$, then $H^{s} \stackrel{d}{\hookrightarrow} h^{k+\alpha}$.

Proof By Sobolev's Embedding Theorem, we know that $H^{s} \hookrightarrow B U C^{k+1}(\mathbb{R})$. Moreover it is well-known that $B U C^{k+1}(\mathbb{R}) \hookrightarrow B U C^{k+\alpha}(\mathbb{R})$ and $\mathcal{S}(\mathbb{R}) \stackrel{d}{\hookrightarrow} H^{s}$. Then,

$$
\mathcal{S}(\mathbb{R}) \stackrel{d}{\hookrightarrow} H^{s} \hookrightarrow B U C^{k+\alpha}(\mathbb{R})
$$

By the definition of little Hölder space, we know that

$$
h^{k+\alpha}=\overline{\mathcal{S}(\mathbb{R})} \quad \text { in } B U C^{k+\alpha}(\mathbb{R}) .
$$

Thanks to Lemma 4.5.4, we get

$$
h^{k+\alpha}=\overline{H^{s}} \quad \text { in } B U C^{k+\alpha}(\mathbb{R})
$$

This means

$$
H^{s} \stackrel{d}{\hookrightarrow} h^{k+\alpha}
$$

Remark 4.5.7. If $\left(\lambda-A_{\widehat{b}}\right)^{-1} \in \mathcal{L}\left(B U C^{1+\alpha}(\mathbb{R}), B U C^{2+\alpha}(\mathbb{R})\right)$ for $\operatorname{Re} \lambda<\omega_{0}$, then $(\lambda-$ $\left.A_{\widehat{b}}\right)^{-1} \in \mathcal{L}\left(h^{1+\alpha}, h^{2+\alpha}\right)$. 
Proof By assumption, we know that $\left(\lambda-A_{\hat{b}}\right)^{-1} \in \mathcal{L}\left(h^{1+\alpha}, B U C^{2+\alpha}(\mathbb{R})\right)$. For every $x \in h^{1+\alpha}$, thanks to Corollary 4.5.6, there exists a sequence $\left(x_{i}\right)$ in $H^{s}(s \geq 4)$ such that $x_{i} \rightarrow x$ in $h^{1+\alpha}$. Thus,

$$
\left(\lambda-A_{\widehat{b}}\right)^{-1} x_{i} \rightarrow\left(\lambda-A_{\widehat{b}}\right)^{-1} x \quad \text { in } B U C^{2+\alpha}(\mathbb{R}) .
$$

Since $\left(\lambda-A_{\hat{b}}\right)^{-1} \in \mathcal{L}\left(H^{s}\right)$, we conclude that

$$
\left(\lambda-A_{\widehat{b}}\right)^{-1} x \text { belongs to the closure of } H^{s} \text { in } B U C^{2+\alpha}(\mathbb{R}) .
$$

Again thanks to Corollary 4.5.6, we have $\left(\lambda-A_{\hat{b}}\right)^{-1} x \in h^{2+\alpha}$.

In the following, we will prove that

$$
\left(\lambda-A_{\widehat{b}}\right)^{-1} \in \mathcal{L}\left(h^{1+\alpha}, h^{2+\alpha}\right)
$$

for every $\lambda \in\left\{z \in \mathbb{C} ; \operatorname{Re}(z)<\omega_{0}\right\}$. Thanks to Remark 4.5.7, we only need to prove that

$$
\left(\lambda-A_{\widehat{b}}\right)^{-1} \in \mathcal{L}\left(B U C^{1+\alpha}(\mathbb{R}), B U C^{2+\alpha}(\mathbb{R})\right)
$$

for every $\lambda \in\left\{z \in \mathbb{C} ; \operatorname{Re}(z)<\omega_{0}\right\}$. To do that, we shall use the theory of Fourier multipliers. Recall that,

$$
\mathcal{M}:=\left(\left\{a \in L_{\infty}\left(\mathbb{R}^{n}\right) ;\left(1+|\xi|^{2}\right)^{|\alpha| / 2} \partial^{\alpha} a \in L_{\infty}\left(\mathbb{R}^{n}\right),|\alpha| \leq[n / 2]+1\right\},\|\cdot\|_{\mathcal{M}}\right),
$$

where

$$
\|a\|_{\mathcal{M}}:=\max _{|\alpha| \leq\left[\frac{n}{2}\right]+1}\left\|\left(1+|\xi|^{2}\right)^{|\alpha| / 2} \partial^{\alpha} a\right\|_{\infty} .
$$

In particular, we only need the case of $n=1$.

Before using the theory of Fourier multipliers, we shall introduce a parameter dependent function. Given $\mu$ and $\beta$, we can define a $C^{\infty}$ function

$$
\varphi_{\mu, \beta}(\xi):=\frac{\cosh \xi}{\mu \cosh \xi-\xi \sinh \xi+\beta} .
$$

Then we can calculate that

$$
\partial_{\xi} \varphi_{\mu, \beta}(\xi)=\frac{\sinh \xi \cosh \xi+\xi+\beta \sinh \xi}{(\mu \cosh \xi-\xi \sinh \xi+\beta)^{2}} \sim O\left(\xi^{-2}\right) \quad \text { as } \xi \rightarrow \infty .
$$

Moreover, as $\xi \rightarrow \infty$,

$$
d\left(1+\xi^{2}\right)^{\frac{1}{2}}=\left(1+\xi^{2}\right)^{-\frac{1}{2}} \xi \sim O(1) .
$$

Thus, we can get the following statement:

Lemma 4.5.8. If Re $\lambda<\omega_{0}$, then $\left(\lambda-A_{\widehat{b}}\right)^{-1} \in \mathcal{L}\left(B U C^{1+\alpha}(\mathbb{R}), B U C^{2+\alpha}(\mathbb{R})\right)$. 
Proof Now we consider two functions

$$
m_{1}(\xi)=\left(\lambda-a_{\widehat{b}}(\xi)\right)^{-1} \quad \text { and } \quad m_{2}(\xi)=\left(1+\xi^{2}\right)^{\frac{1}{2}}\left(\lambda-a_{\widehat{b}}(\xi)\right)^{-1} .
$$

For fixed $\lambda\left(\operatorname{Re} \lambda<\omega_{0}\right)$, then we have

$$
m_{1}(\xi)=c \varphi_{c \lambda, \hat{b}^{\prime}(0)}(c \xi) \quad \text { and } \quad m_{2}(\xi)=c\left(1+\xi^{2}\right)^{\frac{1}{2}} \varphi_{c \lambda, \hat{b}^{\prime}(0)}(c \xi) .
$$

By means of Leibniz' rule, (4.5.3) and (4.5.4), we know that

$$
\partial^{k} m_{1}(\xi) \sim O\left(\xi^{-k-1}\right) \quad \text { and } \quad \partial^{k} m_{2}(\xi) \sim O\left(\xi^{-k}\right) \quad \text { as } \xi \rightarrow \infty \text { for } k=0,1,
$$

i.e. $\left\|m_{1}\right\|_{\mathcal{M}}<\infty$ and $\left\|m_{2}\right\|_{\mathcal{M}}<\infty$. Clearly,

$$
\left(\lambda-A_{\widehat{b}}\right)^{-1}=\mathcal{F}^{-1} m_{1} \mathcal{F} \text { and }(1-\Delta)^{\frac{1}{2}}\left(\lambda-A_{\widehat{b}}\right)^{-1}=\mathcal{F}^{-1} m_{2} \mathcal{F} .
$$

Then, thanks to Corollary 2.3.2, we get that

$$
(1-\Delta)^{\frac{1}{2}}\left(\lambda-A_{\widehat{b}}\right)^{-1} \in \mathcal{L}\left(B U C^{1+\alpha}(\mathbb{R})\right),
$$

and

$$
\left(\lambda-A_{\widehat{b}}\right)^{-1} \in \mathcal{L}\left(B U C^{1+\alpha}(\mathbb{R})\right) .
$$

Since $(1-\Delta)^{\frac{1}{2}} \in I \operatorname{som}\left(B U C^{2+\alpha}(\mathbb{R}), B U C^{1+\alpha}(\mathbb{R})\right)$, we can conclude that

$$
\left(\lambda-A_{\widehat{b}}\right)^{-1} \in \mathcal{L}\left(B U C^{1+\alpha}(\mathbb{R}), B U C^{2+\alpha}(\mathbb{R})\right) .
$$

Thanks to Remark 4.5.7 and Lemma 4.5.8, we get

$$
\left(\lambda-A_{\widehat{b}}\right)^{-1} \in \mathcal{L}\left(h^{1+\alpha}, h^{2+\alpha}\right) \quad \text { for } \operatorname{Re} \lambda<\omega_{0} .
$$

By Lemma 4.5.8, we also know that $\rho\left(A_{\widehat{b}}\right) \supseteq\left\{z \in \mathbb{C} ; \operatorname{Re}(z)<\omega_{0}\right\}$ on $h^{1+\alpha}$. For $0 \leq$ $\omega<\omega_{0}$, we denote that

$$
S_{\omega}=\{z \in \mathbb{C} ; \operatorname{Re}(z) \leq \omega\}
$$

We have known that

$$
\left(\lambda-A_{\widehat{b}}\right)^{-1} \in \mathcal{L}\left(h^{1+\alpha}\right) \quad \text { for } \quad \lambda \in S_{\omega}
$$

so we obtain that

$$
(\lambda-\omega)\left(\lambda-A_{\widehat{b}}\right)^{-1} \in \mathcal{L}\left(h^{1+\alpha}\right) \quad \text { for } \quad \lambda \in S_{\omega} .
$$

Now we consider a function:

$$
m_{\omega}(\lambda, \xi)=(\lambda-\omega)\left(\lambda-a_{\widehat{b}}(\xi)\right)^{-1},
$$

for $\lambda \in S_{\omega}$ and $\xi \in \mathbb{R}$. Clearly

$$
(\lambda-\omega)\left(\lambda-A_{\widehat{b}}\right)^{-1}=\mathcal{F}^{-1} m_{\omega}(\lambda, \xi) \mathcal{F} .
$$




\subsubsection{Estimates of the symbol}

\section{Lemma 4.5.9.}

$$
\left|m_{\omega}(\lambda, \xi)\right|<1 \text { for } \lambda \in S_{\omega}, \xi \in \mathbb{R} .
$$

Proof Since $a_{\widehat{b}}(\xi) \geq \omega_{0}$, then

$$
\operatorname{Re} \lambda-a_{\widehat{b}}(\xi) \leq \operatorname{Re} \lambda-\omega_{0}<\operatorname{Re} \lambda-\omega \leq 0 \quad \text { for } \quad \lambda \in S_{\omega} .
$$

By means of

$$
\left|\lambda-a_{\widehat{b}}(\xi)\right|^{2}=(\operatorname{Im} \lambda)^{2}+\left(\operatorname{Re} \lambda-a_{\widehat{b}}(\xi)\right)^{2},
$$

we get

$$
\left|\lambda-a_{\widehat{b}}(\xi)\right|>|\lambda-\omega| \geq 0
$$

i.e. $\left|m_{\omega}(\lambda, \xi)\right|<1$.

Theorem 4.5.10. There exists a constant $C_{\omega}>0$, such that

$$
|\lambda-\omega| \cdot\left\|\left(\lambda-A_{\widehat{b}}\right)^{-1}\right\|_{\mathcal{L}\left(h^{1+\alpha}\right)} \leq C_{\omega} \quad \text { for } \forall \lambda \in S_{\omega} .
$$

Proof By Corollary 2.3.2, we only need to prove that $\left\|m_{\omega}(\lambda, \xi)\right\|_{\mathcal{M}}$ is independent of $\lambda \in S_{\omega}$. Since $m_{\omega}(\lambda, \xi)$ is an even function for $\xi$, we only need to consider the case $\xi \geq 0$. By direct calculation,

$$
\partial_{\xi} m_{\omega}(\lambda, \xi)=\frac{c^{2}(\lambda-\omega)\left(\cosh (c \xi) \sinh (c \xi)+c \xi+\widehat{b}^{\prime}(0) \sinh (c \xi)\right)}{\left(c \lambda \cosh (c \xi)-c \xi \sinh (c \xi)+\widehat{b}^{\prime}(0)\right)^{2}}
$$

for $\xi \geq 0$ and $\lambda \in S_{\omega}$. Recall that

$$
\lambda-a_{\widehat{b}}(\xi)=\frac{c \lambda \cosh (c \xi)-c \xi \sinh (c \xi)+\widehat{b}^{\prime}(0)}{c \cosh (c \xi)}
$$

and

$$
\left|\lambda-a_{\widehat{b}}(\xi)\right| \geq\left|\operatorname{Re} \lambda-a_{\widehat{b}}(\xi)\right| \geq\left|\omega-\omega_{0}\right| .
$$

Thanks to Lemma 4.5.9 and (4.5.9), we have

$$
\begin{aligned}
Q & :=\left(1+\xi^{2}\right)^{\frac{1}{2}}\left|\partial_{\xi} m_{\omega}(\lambda, \xi)\right| \\
& \leq \frac{c\left(1+\xi^{2}\right)^{\frac{1}{2}}\left|\cosh (c \xi) \sinh (c \xi)+c \xi+\widehat{b}^{\prime}(0) \sinh (c \xi)\right|}{\cosh (c \xi)\left|c \lambda \cosh (c \xi)-c \xi \sinh (c \xi)+\widehat{b}^{\prime}(0)\right|}
\end{aligned}
$$

Now we consider $Q$ with two cases.

Case 1: For $\operatorname{Re} \lambda<0$, recalling that $\widehat{b}^{\prime}(0)<0$, we have

$$
\begin{aligned}
\left|c \lambda \cosh (c \xi)-c \xi \sinh (c \xi)+\widehat{b}^{\prime}(0)\right| & \geq\left|c R e \lambda \cosh (c \xi)-c \xi \sinh (c \xi)+\widehat{b}^{\prime}(0)\right| \\
& \geq c \xi \sinh (c \xi) .
\end{aligned}
$$


Then,

$$
Q \leq \frac{\left(1+\xi^{2}\right)^{\frac{1}{2}}\left|\cosh (c \xi) \sinh (c \xi)+c \xi+\widehat{b}^{\prime}(0) \sinh (c \xi)\right|}{\xi \cosh (c \xi) \sinh (c \xi)}
$$

By (4.5.13), there exist $\xi_{0}>0$ and $C>0$ (independent of $\lambda$ ) such that $Q \leq C$ for $\xi \geq \xi_{0}$. By (4.5.11), we also have

$$
Q \leq \frac{\left(1+\xi^{2}\right)^{\frac{1}{2}}\left|\cosh (c \xi) \sinh (c \xi)+c \xi+\widehat{b}^{\prime}(0) \sinh (c \xi)\right|}{\cosh ^{2}(c \xi)\left|\omega-\omega_{0}\right|}
$$

then there exists $C_{\omega}>0$ such that $Q \leq C_{\omega}$ for $0 \leq \xi \leq \xi_{0}$. Thus $Q$ is bounded independently of $\lambda$.

Case 2: For $\operatorname{Re} \lambda \geq 0$, then $0 \leq \operatorname{Re} \lambda \cosh (c \xi) \leq \omega_{0} \cosh (c \xi)$. Since

$$
\lim _{\xi \rightarrow \infty} \frac{\cosh (c \xi)}{\xi \sinh (c \xi)}=0
$$

there exists $\xi_{1}>0$ such that $\omega_{0} \cosh (c \xi) \leq \frac{\xi \sinh (c \xi)}{2}$ for $\xi>\xi_{1}$. Then, for $\xi>\xi_{1}$, we have

$$
\begin{aligned}
c R e \lambda \cosh (c \xi)-c \xi \sinh (c \xi)+\widehat{b}^{\prime}(0) & \leq c \omega_{0} \cosh (c \xi)-c \xi \sinh (c \xi)+\widehat{b}^{\prime}(0) \\
& \leq-\frac{c}{2} \xi \sinh (c \xi) \leq 0
\end{aligned}
$$

This means $\left|c \lambda \cosh (c \xi)-c \xi \sinh (c \xi)+\widehat{b}^{\prime}(0)\right| \geq \frac{c}{2} \xi \sinh (c \xi)$ for $\xi>\xi_{1}$. Thus, similar to case 1 , we also obtain that $Q$ is bounded independently of $\lambda$.

Therefore, $Q$ is bounded independently of $\lambda$. Furthermore, by Lemma 4.5.9, we get that $\left\|m_{\omega}(\lambda, \xi)\right\|_{\mathcal{M}}$ is independent of $\lambda \in S_{\omega}$.

Theorem 4.5.11. $-A_{\widehat{b}}$ is a sectorial operator, and

$$
\sup \left\{\operatorname{Re} \lambda ; \lambda \in \sigma\left(-A_{\widehat{b}}\right)\right\} \leq-\omega_{0}<0 .
$$

Moreover, $-A_{\widehat{b}} \in \mathcal{H}\left(h^{2+\alpha}, h^{1+\alpha}\right)$.

Proof For every $0<\omega<\omega_{0}$, we denote $\widetilde{S_{\omega}}:=\{\lambda \in \mathbb{C} ; \operatorname{Re} \lambda \geq-\omega\}$. Then it is clear that

$$
\lambda \in \widetilde{S_{\omega}} \text { if and only if }-\lambda \in S_{\omega} .
$$

Then according to (4.5.6), we know $\widetilde{S_{\omega}} \subset \rho\left(-A_{\widehat{b}}\right)$ and

$$
\left(\lambda+A_{\widehat{b}}\right)^{-1}=-\left(-\lambda-A_{\widehat{b}}\right)^{-1} \in \mathcal{L}\left(h^{1+\alpha}, h^{2+\alpha}\right) \quad \text { for } \forall \lambda \in \widetilde{S_{\omega}} .
$$

Thanks to Theorem 4.5.10, there exists a constant $C>0$ such that

$$
|-\lambda-\omega| \cdot\left\|\left(-\lambda-A_{\widehat{b}}\right)^{-1}\right\|_{\mathcal{L}\left(h^{1+\alpha}\right)} \leq C \quad \text { for } \forall \lambda \in \widetilde{S_{\omega}},
$$


i.e.

$$
|\lambda-(-\omega)| \cdot\left\|R\left(\lambda,-A_{\widehat{b}}\right)\right\|_{\mathcal{L}\left(h^{1+\alpha}\right)} \leq C \quad \text { for } \forall \lambda \in \widetilde{S_{\omega}} .
$$

This means $-A_{\widehat{b}}$ is a sectorial operator. Since $\omega$ is arbitrary, (4.5.14) holds true.

Further, by means of (4.5.1) and (4.5.15), we know that

$$
-\omega+A_{\widehat{b}} \in \mathcal{L} i s\left(h^{2+\alpha}, h^{1+\alpha}\right) .
$$

Thus $-A_{\widehat{b}} \in \mathcal{H}\left(h^{2+\alpha}, h^{1+\alpha}\right)$.

Remark 4.5.12. The proof of Theorem 4.5.11 shows that the assumption $\widehat{b}^{\prime}(0)<0$ is only need to get (4.5.14). In particular, we also have $-A_{\widehat{b}} \in \mathcal{H}\left(h^{2+\alpha}, h^{1+\alpha}\right)$ in the case $\widehat{b}^{\prime}(0) \geq 0$.

\subsection{Main results}

\subsubsection{Stability of the transformed problem}

In the next theorem, we shall show the existence of the solution to (4.4.1).

Theorem 4.6.1 (Well-posedness). Assume that $\widehat{b}(0)=0$. Then there exists $r>0$ such that (4.4.1) possesses a classical solution, provided $\left\|g_{0}\right\|_{h^{2+\alpha}}<r$.

Proof Due to Remark 4.5.12, we know

$$
-\partial \Phi_{\widehat{b}}(0) \in \mathcal{H}\left(h^{2+\alpha}, h^{1+\alpha}\right)
$$

Thanks to Corollary 4.4.2 and Proposition 3.1.18, there exists $r \in(0, c)$ such that

$$
-\partial \Phi_{\widehat{b}}(h) \in \mathcal{H}\left(h^{2+\alpha}, h^{1+\alpha}\right) \quad \forall\|h\|_{h^{2+\alpha}}<r .
$$

Now we take $\mathcal{O}:=B(0, r)$ in $h^{2+\alpha}$. Since $r<c$, we can know that $\mathcal{O} \subset h_{\mathfrak{A}}^{2+\alpha}$. By Corollary 4.5.5, we know that $h^{2+\alpha}$ is dense in $h^{1+\alpha}$. Using Corollary 4.4.2 again, we know that (3.3.2) is satisfied. If $\left\|g_{0}\right\|_{h^{2+\alpha}}<r$, then $g_{0} \in \mathcal{O}$. Further, thanks to Theorem 3.3.1, we can know that (4.4.1) possesses a classical solution.

In the following, we study the asymptotic behavior near the equilibrium $g \equiv 0$. Recall that

$$
G(g):=A_{\widehat{b}} g-\Phi_{\widehat{b}}(g) .
$$

Clearly, $G$ is a $C^{\infty}$ function. By the assumption of $\widehat{b}$, we can calculate that $G(0)=$ $0, \partial G(0)=0$. Notice that

$$
\partial_{t} g=-A_{\widehat{b}} g+G(g)
$$

Moreover, we have known that the important condition (4.5.14) is satisfied. Applying the principle of linearized stability([24], Theorem 9.1.2), we obtain that 
Theorem 4.6.2 (Exponential stability). Assume that $\widehat{b}(0)=0$ and $\widehat{b}^{\prime}(0)<0$. For any $\omega \in\left[0, \omega_{0}\right)$, there are positive constants $r$ and $C$, such that for any $g_{0} \in h_{\mathfrak{A}}^{2+\alpha}$ with $\left\|g_{0}\right\|_{h^{2+\alpha}}<r$, we get that the maximal existence interval of the solution $g$ of (4.4.1) is $[0,+\infty)$, and the estimate

$$
\|g(t)\|_{h^{2+\alpha}}+\left\|g^{\prime}(t)\right\|_{h^{1+\alpha}} \leq C e^{-\omega t}\left\|g_{0}\right\|_{h^{2+\alpha}} \quad \forall t \geq 0,
$$

holds.

Thanks to Proposition 4.4.1, we know that problem (4.3.1)-(4.3.6) has a solution $(v, g)$. Since $g(t) \in h_{\mathfrak{A}}^{2+\alpha}$ and $b \in C^{\infty}$, then $\widehat{b}(g(t)) \in C^{2}$. In the following, we will get the estimate of $v$.

Theorem 4.6.3. Assume as Theorem 4.6.2. For any $\omega \in\left[0, \omega_{0}\right)$, there are positive constants $r_{0}$ and $C=C\left(r_{0}\right)$, such that for any $g_{0} \in h_{\mathfrak{A}}^{2+\alpha}$ with $\left\|g_{0}\right\|_{h^{2+\alpha}}<r_{0}$, we get the estimate

$$
\|v(t)\|_{h^{2+\alpha}(\Omega)} \leq C e^{-\omega t}\left\|g_{0}\right\|_{h^{2+\alpha}} \quad \forall t \geq 0,
$$

where $v:=\mathcal{T}(g) g+\mathcal{U}(g) \widehat{b}(g)$.

Proof Let $r$ be the constant in the Theorem 4.6.2. Take $r_{0}<r$, by Theorem 4.6.2, there exist positive constants $M_{1}=M_{1}\left(r_{0}\right)$ and $M_{2}=M_{2}\left(r_{0}\right)$, such that

$$
\|g(t)\|_{h^{2+\alpha}} \leq M_{1} \text { for } \forall t \geq 0 \quad \text { and } \quad\|\widehat{b}\|_{C^{2}\left(\left[-M_{1}, M_{1}\right]\right)} \leq M_{2} .
$$

Since $\widehat{b}(0)=0$, we have

$$
|\widehat{b}(g(t))|=|\widehat{b}(g(t))-\widehat{b}(0)| \leq M_{2}\|g(t)\|_{\infty} .
$$

Again since $\widehat{(b}(g(t, x)))^{\prime}=\widehat{b}^{\prime}(g(t, x)) g^{\prime}(t, x)$, then

$$
\mid \widehat{b}(g(t)))^{\prime} \mid \leq M_{2}\left\|g^{\prime}(t)\right\|_{\infty}
$$

Moreover, for $x \neq y$,

$$
\begin{aligned}
\frac{\left.\mid \widehat{b}(g(t, x)))^{\prime}-\widehat{b}(g(t, y))\right)^{\prime} \mid}{|x-y|^{\alpha}} \leq & \frac{\left|\widehat{b}^{\prime}(g(t, x))\left(g^{\prime}(t, x)-g^{\prime}(t, y)\right)\right|}{|x-y|^{\alpha}} \\
& +\frac{\left.\mid \widehat{b}^{\prime}(g(t, x))-\widehat{b}^{\prime}(g(t, y))\right) g^{\prime}(t, y) \mid}{|x-y|^{\alpha}} \\
\leq & M_{2}\left(\|g(t)\|_{h^{1+\alpha}}+\left\|g^{\prime}(t)\right\|_{\infty} \cdot[g(t)]_{\alpha}\right) .
\end{aligned}
$$

By (4.6.3), (4.6.4) and (4.6.5), there exists a positive constant $C=C\left(M_{1}, M_{2}\right)$ such that

$$
\|\widehat{b}(g(t))\|_{h^{1+\alpha}} \leq C\|g(t)\|_{h^{1+\alpha}} .
$$


By Theorem $4.3 .5 \mathrm{~b}$ ), we know that there exists a positive constant $C_{0}=C_{0}\left(M_{1}\right)$ independent of $t$, such that

$$
\|\mathcal{T}(g(t))\|_{\mathcal{L}\left(h^{2+\alpha}, h^{2+\alpha}(\Omega)\right)} \leq C_{0}, \quad\|\mathcal{U}(g(t))\|_{\mathcal{L}\left(h^{1+\alpha}, h^{2+\alpha}(\Omega)\right)} \leq C_{0} \quad \forall t \geq 0 .
$$

Furthermore, since $h^{2+\alpha} \hookrightarrow h^{1+\alpha}$, by (4.6.1) and (4.6.6), we have

$$
\|v(t)\|_{h^{2+\alpha}(\Omega)} \leq C\left(r_{0}\right)\left(\|g(t)\|_{h^{2+\alpha}}+\|\widehat{b}(g(t))\|_{h^{1+\alpha}}\right) \leq C\left(r_{0}\right) e^{-\omega t}\left\|g_{0}\right\|_{h^{2+\alpha}} \quad \forall t \geq 0 .
$$

\subsubsection{Stability estimates for the interface and pressure}

Thanks to Proposition 4.3.2, we get that problem (4.2.1)-(4.2.6) has a solution $(u, f)$. In the following, we shall get the estimate of $u$. To do it, we need some lemmas and remarks.

Remark 4.6.4. Since $\widehat{b}(g)=-(c+g) b(c+g)$, we can calculate that

$$
\widehat{b}^{\prime}(g)=-b(c+g)-(c+g) b^{\prime}(c+g) .
$$

Therefore,

$$
\left.\begin{array}{r}
\widehat{b}(0)=0 \\
\widehat{b}^{\prime}(0)<0
\end{array}\right\} \Longleftrightarrow\left\{\begin{array}{c}
b(c)=0 \\
b^{\prime}(c)>0
\end{array} .\right.
$$

Lemma 4.6.5. Let $E, F$ and $G$ be Banach spaces, and let $X$ and $Y$ be an open subset of $E$ and $F$, respectively. Let further $0<\alpha<1$. If $h, \tau \in B U C^{\alpha}(X, Y), \psi \in B U C^{\alpha}(Y, G)$, and if $\theta \in C^{1}(X, Y)$ has a bounded derivative, then

$$
\phi:=\psi \circ \theta \in B U C^{\alpha}(X, G), \quad \rho:=h \cdot \tau \in B U C^{\alpha}(X, Y) .
$$

In particular,

$$
[\phi]_{\alpha} \leq[\psi]_{\alpha}\left\|\theta^{\prime}\right\|_{\infty}^{\alpha}, \quad[\rho]_{\alpha} \leq[h]_{\alpha}\|\tau\|_{\infty}+\|h\|_{\infty}[\tau]_{\alpha} .
$$

Proof It is obvious for the function $\rho$. Therefore, we only need prove the case of function $\phi$. By assumptions, we easily know that $\phi \in B C(X, G)$. For $\forall x, y \in X$,

$$
\begin{aligned}
\|\phi(x)-\phi(y)\|_{G} & =\|\psi(\theta(x))-\psi(\theta(y))\|_{G} \\
& \leq[\psi]_{\alpha}\|\theta(x)-\theta(y)\|_{F}^{\alpha} \\
& \leq[\psi]_{\alpha}\left(\left\|\theta^{\prime}\right\|_{\infty}\|x-y\|_{E}\right)^{\alpha} \\
& \leq[\psi]_{\alpha}\left\|\theta^{\prime}\right\|_{\infty}^{\alpha}\|x-y\|_{E}^{\alpha} .
\end{aligned}
$$

Thus, $\phi \in B U C^{\alpha}(X, G)$ and $[\phi]_{\alpha} \leq[\psi]_{\alpha}\left\|\theta^{\prime}\right\|_{\infty}^{\alpha}$. 
Lemma 4.6.6. If $g \in h_{\mathfrak{A}}^{2+\alpha}$, then $\varphi_{g} \in C^{2+\alpha}\left(\Omega_{f}, \Omega\right)$ and $\partial_{k} \varphi_{g} \in B U C^{1+\alpha}\left(\Omega_{f}, \mathbb{R}^{2}\right)(k=$ $1,2)$. Furthermore, $\left\|\partial_{k} \varphi_{g}\right\|_{B U C^{1+\alpha}\left(\Omega_{f}, \mathbb{R}^{2}\right)}$ only depends on $\|g\|_{h^{2+\alpha}}$ and constant $M$, where $M:=\inf _{x \in \mathbb{R}}\{c+g(x)\}$.

Proof Denote the $k$ th component of $\varphi_{g}$ by $\varphi_{k}(k=1,2)$, i.e. $\varphi_{g}=\left(\varphi_{1}, \varphi_{2}\right)$. In order to prove $\varphi_{g} \in C^{2+\alpha}$, it is sufficient to prove that $\varphi_{1}, \varphi_{2} \in C^{2+\alpha}$. By the definition of $\mathfrak{A}$, there exists a positive constant $M$ such that $\inf _{x \in \mathbb{R}}\{c+g(x)\} \geq M$. Moreover, we can calculate that

$$
\begin{aligned}
& \partial \varphi_{1}(x, y)=(1,0) \\
& \partial^{2} \varphi_{1}(x, y)=\left(\begin{array}{ll}
0 & 0 \\
0 & 0
\end{array}\right), \quad \partial^{2} \varphi_{2}(x, y)=\left(\begin{array}{cc}
\frac{y g^{\prime \prime}(x)}{(c+g(x))^{2}}-\frac{2 g^{\prime 2}(x) y}{(c+g(x))^{3}} & \frac{g^{\prime}(x)}{(c+g(x))^{2}} \\
\frac{g^{\prime}(x)}{(c+g(x))^{2}} & 0
\end{array}\right) .
\end{aligned}
$$

Moreover,

$$
\begin{aligned}
&\left|\frac{y g^{\prime}(x)}{(c+g(x))^{2}}\right| \leq \frac{\left\|g^{\prime}\right\|_{\infty}}{M},\left|\frac{1}{c+g(x)}\right| \leq \frac{1}{M}, \\
&\left|\frac{y g^{\prime \prime}(x)}{(c+g(x))^{2}}-\frac{2 g^{\prime 2}(x) y}{(c+g(x))^{3}}\right| \leq \frac{\left\|g^{\prime \prime}\right\|_{\infty}}{M}+\frac{2\left\|g^{\prime}\right\|_{\infty}^{2}}{M^{2}}, \quad\left|\frac{g^{\prime}(x)}{(c+g(x))^{2}}\right| \leq \frac{\left\|g^{\prime}\right\|_{\infty}}{M^{2}} .
\end{aligned}
$$

For $0<\sqrt{\left(x_{1}-x_{2}\right)^{2}+\left(y_{1}-y_{2}\right)^{2}}<\frac{1}{2}$, we have

$$
\begin{aligned}
& \left|\frac{y_{1} g^{\prime \prime}\left(x_{1}\right)}{\left(c+g\left(x_{1}\right)\right)^{2}}-\frac{y_{2} g^{\prime \prime}\left(x_{2}\right)}{\left(c+g\left(x_{2}\right)\right)^{2}}\right| \\
\leq & \frac{1}{M^{4}} \mid\left(y_{1}-y_{2}\right) g^{\prime \prime}\left(x_{1}\right)\left(c+g\left(x_{2}\right)\right)^{2}+y_{2}\left(g^{\prime \prime}\left(x_{1}\right)-g^{\prime \prime}\left(x_{2}\right)\right)\left(c+g\left(x_{2}\right)\right)^{2} \\
& +y_{2} g^{\prime \prime}\left(x_{2}\right)\left(2 c+g\left(x_{1}\right)+g\left(x_{2}\right)\right)\left(g\left(x_{2}\right)-g\left(x_{1}\right)\right) \mid \\
\leq & \frac{1}{M^{4}}\left[\left\|g^{\prime \prime}\right\|_{\infty}\left(c+\|g\|_{\infty}\right)^{2}\left|y_{1}-y_{2}\right|+\|g\|_{h^{2+\alpha}}\left(c+\|g\|_{\infty}\right)^{3}\left|x_{1}-x_{2}\right|^{\alpha}\right. \\
& \left.+2\left\|g^{\prime \prime}\right\|_{\infty}\left(c+\|g\|_{\infty}\right)^{2}\left\|g^{\prime}\right\|_{\infty}\left|x_{1}-x_{2}\right|\right] \\
\leq & C\left(\left|x_{1}-x_{2}\right|^{\alpha}+\left|y_{1}-y_{2}\right|^{\alpha}\right) \\
\leq & 2 C\left[\left(x_{1}-x_{2}\right)^{2}+\left(y_{1}-y_{2}\right)^{2}\right]^{\frac{\alpha}{2}}
\end{aligned}
$$


and

$$
\begin{aligned}
& \left|\frac{g^{\prime 2}\left(x_{1}\right) y_{1}}{\left(c+g\left(x_{1}\right)\right)^{3}}-\frac{g^{\prime 2}\left(x_{2}\right) y_{2}}{\left(c+g\left(x_{2}\right)\right)^{3}}\right| \\
\leq & \frac{1}{M^{6}} \mid\left(y_{1}-y_{2}\right) g^{\prime 2}\left(x_{1}\right)\left(c+g\left(x_{2}\right)\right)^{3}+y_{2}\left(g^{\prime}\left(x_{1}\right)-g^{\prime}\left(x_{2}\right)\right)\left(g^{\prime}\left(x_{1}\right)+g^{\prime}\left(x_{2}\right)\right) . \\
& \left(c+g\left(x_{2}\right)\right)^{3}+y_{2} g^{\prime 2}\left(x_{2}\right)\left\{\left(c+g\left(x_{1}\right)\right)^{2}+\left(c+g\left(x_{1}\right)\right)\left(c+g\left(x_{2}\right)\right)\right. \\
& \left.+\left(c+g\left(x_{2}\right)\right)^{2}\right\}\left(g\left(x_{2}\right)-g\left(x_{1}\right)\right) \mid \\
\leq & \frac{1}{M^{6}}\left(\left\|g^{\prime}\right\|_{\infty}^{2}\left(c+\|g\|_{\infty}\right)^{3}\left|y_{1}-y_{2}\right|+2\left\|g^{\prime \prime}\right\|_{\infty}\left\|g^{\prime}\right\|_{\infty}\left(c+\|g\|_{\infty}\right)^{4}\left|x_{1}-x_{2}\right|\right. \\
& \left.+3\left\|g^{\prime}\right\|_{\infty}^{3}\left(c+\|g\|_{\infty}\right)^{3}\left|x_{1}-x_{2}\right|\right) \\
\leq & C\left(\left|x_{1}-x_{2}\right|^{\alpha}+\left|y_{1}-y_{2}\right|^{\alpha}\right) \\
\leq & 2 C\left[\left(x_{1}-x_{2}\right)^{2}+\left(y_{1}-y_{2}\right)^{2}\right]^{\frac{\alpha}{2}} .
\end{aligned}
$$

For $\sqrt{\left(x_{1}-x_{2}\right)^{2}+\left(y_{1}-y_{2}\right)^{2}} \geq \frac{1}{2}$, we also have

$$
\begin{aligned}
& \left|\frac{y_{1} g^{\prime \prime}\left(x_{1}\right)}{\left(c+g\left(x_{1}\right)\right)^{2}}-\frac{y_{2} g^{\prime \prime}\left(x_{2}\right)}{\left(c+g\left(x_{2}\right)\right)^{2}}\right| \leq \frac{2\left\|g^{\prime \prime}\right\|_{\infty}}{M} \leq \frac{\left\|g^{\prime \prime}\right\|_{\infty}}{M} \cdot\left[\left(x_{1}-x_{2}\right)^{2}+\left(y_{1}-y_{2}\right)^{2}\right]^{\frac{\alpha}{2}} \\
& \left|\frac{g^{\prime 2}\left(x_{1}\right) y_{1}}{\left(c+g\left(x_{1}\right)\right)^{3}}-\frac{g^{\prime 2}\left(x_{2}\right) y_{2}}{\left(c+g\left(x_{2}\right)\right)^{3}}\right| \leq \frac{2\left\|g^{\prime}\right\|_{\infty}^{2}}{M^{2}} \leq \frac{4\left\|g^{\prime}\right\|_{\infty}^{2}}{M^{2}} \cdot\left[\left(x_{1}-x_{2}\right)^{2}+\left(y_{1}-y_{2}\right)^{2}\right]^{\frac{\alpha}{2}} .
\end{aligned}
$$

Thus, $\varphi_{1}, \varphi_{2} \in C^{2+\alpha}$ and $\partial_{i} \varphi_{1}, \partial_{i} \varphi_{2} \in B U C^{1+\alpha}\left(\Omega_{f}, \mathbb{R}\right)(i=1,2)$. Furthermore, $\left\|\partial_{i} \varphi_{1}\right\|_{B U C^{1+\alpha}\left(\Omega_{f}, \mathbb{R}\right)}$, and $\left\|\partial_{i} \varphi_{2}\right\|_{B U C^{1+\alpha}\left(\Omega_{f}, \mathbb{R}\right)}$, only depend on $\|g\|_{h^{2+\alpha}}$ and $M$. Finally, since $\partial_{i} \varphi_{g}=\left(\partial_{i} \varphi_{1}, \partial_{i} \varphi_{2}\right)$, then $\partial_{i} \varphi_{g} \in B U C^{1+\alpha}\left(\Omega_{f}, \mathbb{R}^{2}\right)$, and $\left\|\partial_{i} \varphi_{g}\right\|_{B U C^{1+\alpha}\left(\Omega_{f}, \mathbb{R}^{2}\right)}$ only depends on $\|g\|_{h^{2+\alpha}}$ and $M$.

Remark 4.6.7. Similarly, we also can calculate that $\varphi_{g}^{-1} \in C^{2+\alpha}\left(\Omega, \Omega_{f}\right)$ and $\partial_{k} \varphi_{g}^{-1} \in$ $B U C^{1+\alpha}\left(\Omega, \mathbb{R}^{2}\right)(k=1,2)$. Furthermore, $\left\|\partial_{k} \varphi_{g}^{-1}\right\|_{B U C^{1+\alpha}\left(\Omega, \mathbb{R}^{2}\right)}$ only depends on $\|g\|_{h^{2+\alpha}}$. Hence, we can obtain that the following assertion. If $\psi:=\phi \circ \varphi_{g}$, then we have

$$
\psi \in B U C^{2+\alpha}\left(\Omega_{f}\right) \quad \text { iff } \quad \phi \in B U C^{2+\alpha}(\Omega) .
$$

Furthermore, there exist $C_{1}, C_{2}>0$ (only depend on $M$ and $\|g\|_{h^{2+\alpha}}$ ) such that

$$
C_{1}\|\phi\|_{B U C^{2+\alpha}(\Omega)} \leq\|\psi\|_{B U C^{2+\alpha}\left(\Omega_{f}\right)} \leq C_{2}\|\phi\|_{B U C^{2+\alpha}(\Omega)},
$$

where $M$ is defined by Lemma 4.6.6.

Proof $" \Longleftarrow "$ Suppose $\phi \in B U C^{2+\alpha}(\Omega)$ and $\varphi_{g}=\left(\varphi_{1}, \varphi_{2}\right)$. Thanks to Lemma 4.6.5, we know that $\varphi_{g} \in C^{2+\alpha}\left(\Omega_{f}, \Omega\right)$. Then we have $\psi \in B C^{2}\left(\Omega_{f}\right)$. By chain law, we can know that $\|\psi\|_{\infty} \leq\|\phi\|_{\infty}$ and for $j, k \in\{1,2\}$,

$$
\begin{aligned}
\partial_{k} \psi & =\sum_{i=1}^{2} \partial_{i} \phi\left(\varphi_{g}\right) \cdot \partial_{k} \varphi_{i}=\sum_{i=1}^{2}\left(\left(\partial_{i} \phi\right) \circ \varphi_{g}\right) \cdot \partial_{k} \varphi_{i} \\
\partial_{j} \partial_{k} \psi & =\sum_{i, l=1}^{2} \partial_{l} \partial_{i} \phi\left(\varphi_{g}\right) \cdot \partial_{j} \varphi_{l} \cdot \partial_{k} \varphi_{i}+\sum_{i=1}^{2} \partial_{i} \phi\left(\varphi_{g}\right) \cdot \partial_{j} \partial_{k} \varphi_{i} \\
& =\sum_{i, l=1}^{2}\left(\left(\partial_{l} \partial_{i} \phi\right) \circ \varphi_{g}\right) \cdot \partial_{j} \varphi_{l} \cdot \partial_{k} \varphi_{i}+\sum_{i=1}^{2}\left(\left(\partial_{i} \phi\right) \circ \varphi_{g}\right) \cdot \partial_{j} \partial_{k} \varphi_{i} .
\end{aligned}
$$


Again by Lemma 4.6.6 and Lemma 4.6.5, we can conclude that $\partial_{j} \partial_{k} \psi \in B U C^{\alpha}\left(\Omega_{f}\right)$. This implies $\psi \in B U C^{2+\alpha}\left(\Omega_{f}\right)$. Moreover, there exists a constant $C_{2}>0$ (only depends on $M$ and $\|g\|_{h^{2+\alpha}}$ ) such that

$$
\|\psi\|_{B U C^{2+\alpha}\left(\Omega_{f}\right)} \leq C_{2}\|\phi\|_{B U C^{2+\alpha}(\Omega)}
$$

$" \Longrightarrow$ "By Lemma 4.3.1, $\varphi_{g} \in \operatorname{Diff} f^{2+\alpha}\left(\Omega_{f}, \Omega\right)$. Similar to Lemma 4.6.6, we can get the similar properties for $\varphi_{g}^{-1}$. Therefore the statement can be obtained similarly. Moreover, there exists a constant $C>0$ (only depends on $\|g\|_{h^{2+\alpha}}$ ) such that

$$
\|\phi\|_{B U C^{2+\alpha}(\Omega)} \leq C\|\psi\|_{B U C^{2+\alpha}\left(\Omega_{f}\right)} .
$$

Take $C_{1}=\frac{1}{C}$, we can get the estimate (4.6.10).

Corollary 4.6.8. Given $g \in h_{\mathfrak{A}}^{2+\alpha}$. If $\psi=\phi \circ \varphi_{g}$, then $\psi \in h^{2+\alpha}\left(\Omega_{f}\right)$ iff $\phi \in h^{2+\alpha}(\Omega)$.

Proof By Remark 4.6.7, we have known that $\psi \in B U C^{2+\alpha}\left(\Omega_{f}\right)$ iff $\phi \in B U C^{2+\alpha}(\Omega)$. For $\phi \in h^{2+\alpha}(\Omega)$, there exists a sequence $\left\{\phi_{i}\right\} \in \gamma_{\Omega} \mathcal{S}\left(\mathbb{R}^{2}\right)$ such that

$$
\phi_{i} \rightarrow \phi \quad \text { in } \quad B U C^{2+\alpha}(\Omega) \quad \text { as } \quad i \rightarrow \infty \text {. }
$$

Since $g \in h_{\mathfrak{A}}^{2+\alpha}$ and Remark 4.2.1, there also exists a sequence $\left\{g_{i}\right\} \in \mathcal{S}(\mathbb{R}) \cap \mathfrak{A}$ such that

$$
g_{i} \rightarrow g \quad \text { in } \quad B U C^{2+\alpha}(\mathbb{R}) \quad \text { as } \quad i \rightarrow \infty .
$$

Thanks to Lemma 4.3.1, we know $\varphi_{g_{i}} \in \operatorname{Diff} f^{\infty}\left(\Omega_{f}, \Omega\right)$. Thus $\phi_{i} \circ \varphi_{g_{i}} \in \gamma_{\Omega_{f}} \mathcal{S}\left(\mathbb{R}^{2}\right)$. Moreover,

$$
\phi_{i} \circ \varphi_{g_{i}} \rightarrow \phi \circ \varphi_{g} \quad \text { in } \quad B U C^{2+\alpha}\left(\Omega_{f}\right) \quad \text { as } \quad i \rightarrow \infty
$$

This implies $\psi \in h^{2+\alpha}\left(\Omega_{f}\right)$. The converse is similar.

Theorem 4.6.9. Assume that $b(c)=0$ and $b^{\prime}(c)>0$. For any $\omega \in\left[0, \omega_{0}\right)$, there exist positive constants $r_{1}$ and $C=C\left(r_{1}\right)$, such that for any $f_{0}-c \in h_{\mathfrak{A}}^{2+\alpha}$ with $\left\|f_{0}-c\right\|_{h^{2+\alpha}}<$ $r_{1}$, the problem (4.2.1)-(4.2.6) has a unique global solution $(u(t, x, y), f(t, x))$ such that $u-c \in C\left([0,+\infty), h^{2+\alpha}\left(\Omega_{f(t)}\right)\right)$, and $f-c \in C\left([0,+\infty), h_{\mathfrak{A}}^{2+\alpha}\right)$. Furthermore, we get the estimate

$$
\|f(t)-c\|_{h^{2+\alpha}}+\left\|f^{\prime}(t)\right\|_{h^{1+\alpha}}+\|u(t)-c\|_{h^{2+\alpha}\left(\Omega_{f(t)}\right)} \leq C e^{-\omega t}\left\|f_{0}-c\right\|_{h^{2+\alpha}}, \quad \forall t \geq 0 .
$$

Proof Let $r, r_{0}$ be the constants in the Theorem 4.6.2 and Theorem 4.6.3 respectively, and $g_{0}:=f_{0}-c$. Take $r_{1} \leq \min \left\{r, r_{0}\right\}$, by Remark 4.6.4, Theorem 4.6 .2 and Theorem 4.6.3, we know that if $f_{0}-c \in h_{\mathfrak{A}}^{2+\alpha}$ with $\left\|f_{0}-c\right\|_{h^{2+\alpha}}<r_{1}$, then we have

$$
g \in C\left([0,+\infty), h_{\mathfrak{A}}^{2+\alpha}\right) \cap C^{1}\left([0,+\infty), h^{1+\alpha}\right) \text { and } v \in C\left([0,+\infty), h^{2+\alpha}(\Omega)\right) .
$$


Moreover, there exists a constant $C_{0}>0$ such that

$$
\|g(t)\|_{h^{2+\alpha}}+\left\|g^{\prime}(t)\right\|_{h^{1+\alpha}}+\|v\|_{h^{2+\alpha}(\Omega)} \leq C_{0} e^{-\omega t}\left\|f_{0}-c\right\|_{h^{2+\alpha}}, \quad \forall t \geq 0 .
$$

Thanks to Proposition 4.4.1 and Proposition 4.3.2, we get that problem (4.2.1)-(4.2.6) exists a unique solution $(u(t, x, y), f(t, x))=\left(v\left(t, \varphi_{g(t, x)}(x, y)\right)+c, g(t, x)+c\right)$. Due to (4.6.12), we know $\|g(t)\|_{h^{2+\alpha}} \leq C_{0} r_{1}$ for $\forall t \geq 0$. Let $M(t):=\inf _{x \in \mathbb{R}}\{c+g(t, x)\}$, and we take $r_{1}$ small enough such that $r_{1}<\frac{c}{2 C_{0}}$, then we can know that

$$
M(t) \geq c-\|g(t)\|_{\infty} \geq \frac{c}{2} \quad \text { for } \forall t \geq 0 .
$$

It is similar to Remark 4.6.7, we can get $u(t) \in B U C^{2+\alpha}\left(\Omega_{f(t)}\right)$ and there exists $C_{1}=$ $C_{1}\left(r_{1}\right)>0$ (independent of $t$ ) such that

$$
\|u(t)-c\|_{B U C^{2+\alpha}\left(\Omega_{f(t)}\right)} \leq C_{1}\|v(t)\|_{h^{2+\alpha}(\Omega)} \quad \text { for } \forall t \geq 0 .
$$

By Corollary 4.6.8, $u(t)-c \in h^{2+\alpha}\left(\Omega_{f(t)}\right)$. Let $C=C_{0}\left(C_{1}+1\right)$, by (4.6.13) and (4.6.12), we get

$$
\|f(t)-c\|_{h^{2+\alpha}}+\left\|f^{\prime}(t)\right\|_{h^{1+\alpha}}+\|u(t)-c\|_{h^{2+\alpha}\left(\Omega_{f(t)}\right)} \leq C e^{-\omega t}\left\|f_{0}-c\right\|_{h^{2+\alpha}} \quad \text { for } \forall t \geq 0 .
$$

Remark 4.6.10. By Darcy's law $\mathbf{V}_{w}=-\nabla u$, the $y$ component of the velocity $\mathbf{V}_{w}$ of the flow on the fixed layer can be pictured as the following (see Figure 3):

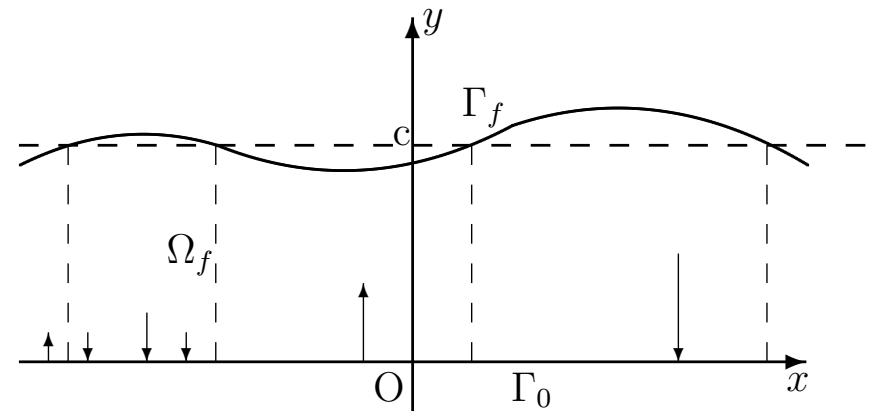

Figure 3

This implies that when the interface function $f$ is larger than the constant $c$, the external source layer will supply an instant negative injection rate which causes the interface to fall. On the other hand, when the interface function $f$ is less than the constant $c$, the external source layer will supply an instant positive injection rate which leads the interface to increase. Thus $(u, f) \equiv(c, c)$ is a stationary solution of problem (4.2.1)-(4.2.6). 


\section{Bibliography}

[1] F. Abergel, Well-posedness for a Cauchy problem associated to timedependent free boundaries with nonlocal leading terms, Comm. PDE, 21 (1996), No. 9-10, 1307-1319.

[2] S. Agmon, A. Douglis, L. Nirenberg, Estimates near the boundary for solutions of elliptic partial differential equations satisfying general boundary conditions. I, Comm. Pure Appl. Math. 12 (1959), 623-727.

[3] H. Amann, Ordinary Differential Equations. An Introduction to Nonlinear Analysis, W. de Gruyter, Berlin, 1990.

[4] H. Amann, Linear and Quasilinear Parabolic Problems, Birkhäuser Verlag, Basel, 1995.

[5] J. Bear \& Y. Bachmat, Introduction to Modeling of Transport Phenomena in Porous Media, Kluwer Academic Publisher, Boston, 1990.

[6] J. Bergh, J. Löfström, Interpolation Spaces. An Introduction, Springer Verlag, Berlin, 1976.

[7] G. I. Bizhanova \& V. A. Solonnikov, On the initial boundary value problems for second order parabolic equations with time derivative in the boundary and conjugate conditions in weighted Hölder spaces of functions, Abstract ICM 94, 164 (1994).

[8] G. I. Bizhanova, Solution in a weighted Hölder function space of multidimensional two-phase Stefan and Florin problems for a second order parabolic equation in a bounded domain, Algebra Analiz 7 (1995), No. 2, 46-76.

[9] G. Da Prato, E. Sinestrari, Hölder regularity for nonautonomous abstract parabolic equations, Israel J. Math. 42 (1982), 1-19.

[10] G. Da Prato, E. Sinestrari, Differential operators with nondense domain, Ann. Sc. Norm. Sup. Pisa 14 (1987), 285-344.

[11] J. Escher \& G. Simonett, On a multi-dimensional moving boundary prob-

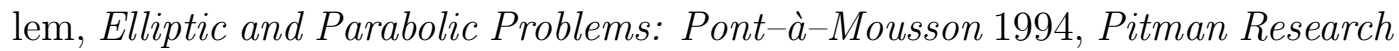
Notes in Math. Longman, Essex, 325, (1995) 98-105. 
[12] J. Escher \& G. Simonett, Maximal regularity for a free boundary problem, Nonlinear Differential Equations Appl. 2, (1995), No. 4, 463-510.

[13] J. Escher \& G. Simonett, Analyticity of the interface in a free boundary problem, Math. Ann. 305, (1996) 439-459.

[14] J. Escher, On moving boundaries in deformable media, Adv. Math. Sci. Appl. 7 (1997), No. 1, 275-316.

[15] J. Escher \& G. Simonett, Moving surfaces and abstract parabolic evolution equations, Progr. Nonlinear Differential Equations Appl. Vol. 35, 183-212, Birkhäuser, Basel, 1999.

[16] J. Escher \& G. Prokert, Stability of the equilibria for spatially periodic flows in porous media, Nonlinear Analysis, TMA 45 (2001) 1061-1080.

[17] H. O. Fattorini, The Cauchy Problem. Addison-Wesley, Reading,Mass., 1983.

[18] A. Friedman \& B. Hu, The Stefan problem with kinetic condition at the free boundary, Ann. Scuola Norm. Pisa,(4) XLIV, (1992) 87-111.

[19] G. H. Hardy, J. E. Littlewood, G. Pòlya, Inequalities, Cambridge Univ. Press, Cambridge, 1934.

[20] H.S. Hele-Shaw, The flow of water, Nature, 58 (1898), 34-36.

[21] L. Hörmander, The Analysis of Linear Partial Differentail Operators, I-IV. Springer Verlag, Berlin, 1983, 1985.

[22] H. Kawarada \& H. Koshigoe, Unsteady flow in porous media with a free surface, Japan J. Indust. Appl. Math. 8 (1991), 41-82.

[23] J. L. Lions, Théorèmes de trace et d'interpolation, (I)-(V); (I),(II) Ann. Sc. Norm. Sup. Pisa 13 (1959), 389-403; 14 (1960), 317-331; (III) J. Math. Pura Appl. 42 (1963), 195-203; (IV) Math. Ann. 151 (1963), 42-56; (V) An. Acad. Brasil. Ci. 35 (1963), 1-10.

[24] A. Lunardi, Analytic Semigroups and Optimal Regularity in Parabolic Problems, Birkhäuser Verlag, Basel, 1995.

[25] V. A. Solonnikov, Free boundary problems for second order parabolic equations, Progress in partial differential equations, Vol. 2 (Pont-à-Mousson,1997), 116-126, Pitman Res. Notes Math. Ser., 384, Longman, Harlow, 1998.

[26] V. A. Solonnikov \& E. V. Frolova, $L_{p}$-theory for the Stefan problem, J. Math. Sci. 99 (2000), No. 1, 989-1006. 
[27] E.M. Stein, Singular Integrals and Differentiability Properties of Functions, Princeton Univ. Press, Priceton, N.J., 1970.

[28] H. Triebel, Höhere Analysis, VEB Deutscher Verlag der Wissenschatfen, Berlin, 1972.

[29] H. TRIEBEL, Interpolation Theory, Function Spaces, Differential Operators, North Holland, Amsterdam, 1978. 